\title{
Formal homo-Nazarov and other Cyclizations Reactions of Activated Cyclopropanes
}

\author{
Filippo De Simone, Tanguy Saget, Fides Benfatti, Sofia Almeida and Jérôme Waser*[a]
}

Dedication ((optional))

\begin{abstract}
The Nazarov cyclization of divinyl ketones gives access to cyclopentenones. Replacing one of the vinyl groups by a cyclopropane leads to a formal homo-Nazarov process for the synthesis of cyclohexenones. In contrast to the Nazarov reaction, the cyclization of vinyl-cyclopropyl ketones is a stepwise process, often requiring harsh conditions. Herein, we describe two different approaches for further polarization of the threemembered ring of vinyl-cyclopropyl ketones in order to allow the formal homo-Nazarov reaction under mild catalytic conditions. In the first
\end{abstract}

approach, the introduction of an ester group $\alpha$ to the carbonyl on the cyclopropane gave a more than tenfold increase in reaction rate, allowing us to extend the scope of the reaction to non electron-rich aryl donor substituents in $\beta$ position to the carbonyl on the cyclopropane. In this case, a proof of principle for asymmetric induction could be achieved using chiral Lewis acid catalysts. In the second approach, heteroatoms, especially nitrogen, were introduced $\beta$ to the carbonyl on the cyclopropane. In this case, the reaction was especially successful when the vinyl group was replaced by an indole heterocycle. With a free indole, the formal homo-Nazarov cyclization on the $\mathrm{C} 3$ position of indole was observed using a copper catalyst. In contrast, a new cyclization reaction on the $\mathrm{N} 1$ position was observed with Brønsted acid catalysts. Both reactions were applied to the synthesis of natural alkaloids. Preliminary investigations on the rationalization of the observed regioselectivity are also reported.

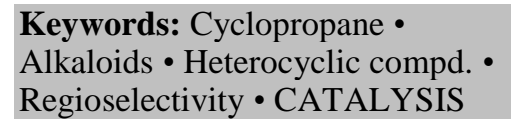

\section{Introduction}

The continuing progress in medicinal chemistry and chemical biology requires flexible synthetic approaches for the generation of libraries of active molecules and their analogues. A broad range of biologically active natural and synthetic compounds displays a complex polycyclic heterocyclic scaffold as core structure. Consequently, the search for new cyclization and cycloaddition reactions is a fundamental task in organic chemistry. ${ }^{[1]}$

Pericyclic reactions involving the concerted rearrangement of bonding electrons have the advantage of low activation barriers, allowing mild reaction conditions. The Nazarov cyclization,

[a] F. De Simone, T. Saget, Dr. F. Benfatti, S. Almeida and Prof. Dr. J. Waser

Laboratory of Catalysis and Organic Synthesis

Ecole Polytechnique Fédérale de Lausanne

EPFL SB ISIC LCSO, BCH 4306, 1015 Lausanne $(\mathrm{CH})$

Fax: (+)41216939700

E-mail: jerome.waser@epfl.ch

Homepage: http://lcso.epfl.ch/

Supporting information for this article is available on the WWW under http://www.chemeurj.org/ or from the author. discovered by Nazarov in $1941,{ }^{[2]}$ is an important example of an electrocyclic ring closure - a subclass of pericyclic reactions- in which five-membered rings are generated starting from divinyl (or arylvinyl) ketones (Scheme 1, A). After activation of the carbonyl with a Brønsted or a Lewis acid, the intermediate pentadienyl cation I undergoes a conrotatory ring closure generating a cyclopentenone after proton transfer. For several decades, the Nazarov cyclization was limited by the necessity to use a stoichiometric amount of a strong Lewis or Brønsted acid. ${ }^{[3]}$ In the last years however, several groups have reported milder catalytic methods. ${ }^{[4-5]}$ Key for success was the introduction of an electron-donating and an electronwithdrawing group in $\alpha$ position to the carbonyl (EDG and $\mathrm{R}^{3}$ in Scheme 1). In such substrates, one of the vinyl groups becomes nucleophilic, and the other one electrophilic (cross polarization), resulting in an enhanced reactivity of the divinyl ketone. This approach was extensively investigated by Frontier and coworkers. ${ }^{[4 k]}$ Since this seminal discovery, several catalytic versions were reported, together with the first examples of asymmetric induction. ${ }^{[5]}$

Starting from the Nazarov cyclization, a possible approach to access larger ring systems consists in the substitution of a double bond by an activated cyclopropyl group, resulting in a homoNazarov process (Scheme 1, B). The ring strain of cyclopropanes make them excellent precursors of reactive intermediates in the formation of new carbon-carbon bonds. Furthermore, the Walsh orbital description of the three-membered ring has been used to 
illustrate the partial $\pi$-character of C-C bonds in cyclopropanes. ${ }^{[6]}$ They can consequently be considered as homologues of olefins in several annulations and cyclization reactions. Nevertheless, there is strong evidence that all the cyclizations of vinyl-cyclopropyl ketones reported so far are stepwise processes in contrast to the concerted Nazarov reaction (Scheme 1, C) and can consequently only formally be compared with an electrocyclic process. ${ }^{[7]}$ An important consequence from the stepwise mechanism is that the reaction is usually not anymore conrotatory. However, as starting materials and products are identical in reactions $\mathbf{B}$ and $\mathbf{C}$, it is useful to keep a "homo-Nazarov" process in mind for retrosynthetic planning. The term formal should never been omitted, as it serves as a strong warning sign to indicate a different mechanism. Finally, further reactions involving intramolecular attack of a nucleophile $\beta$ to the carbonyl on the cyclopropane, although highly useful, should not be called formal homo-Nazarov reactions, as they lack the pattern of $\pi$ bonds characteristic for the reaction (Scheme 1, D). ${ }^{[7 \mathrm{~g}, 8]}$

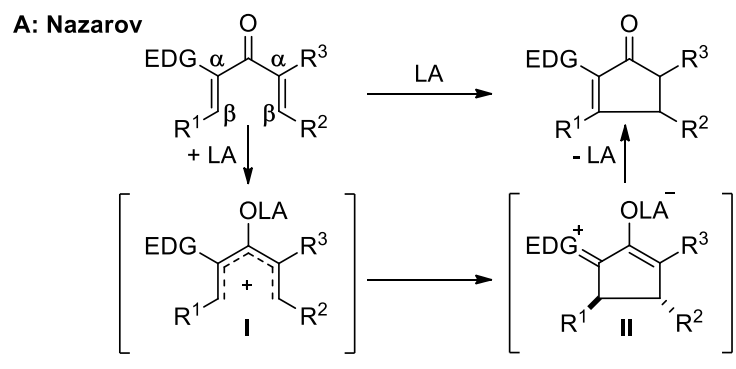

\section{B: Homo-Nazarov}

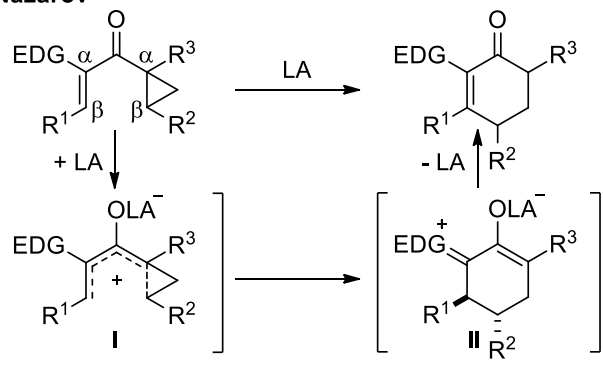

\section{C: Formal Homo-Nazarov}

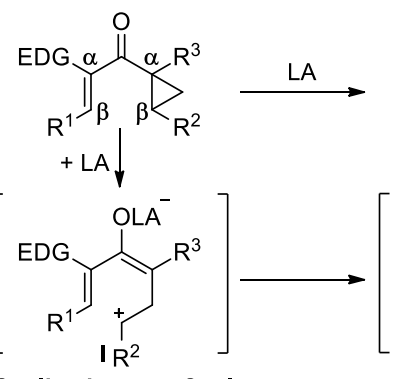

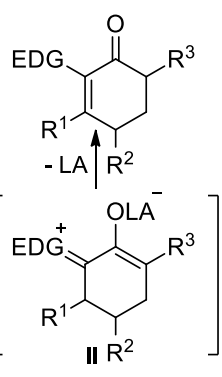

D: Other Cyclizations on Cyclopropanes<smiles>[R]C1CC([R])C(=O)CN1</smiles>

Scheme 1. Nazarov cyclization of divinyl ketones and cyclization reactions of vinylcyclopropyl ketones. $(\mathrm{EDG}=$ electron-donating group, LA = Lewis Acid).

To enhance the reactivity of cyclopropanes in order to allow cyclization reactions under mild conditions, it is often required to add further activating groups onto the ring. In particular, zwitterionic intermediates can be generated under mild conditions by tuning the donor-acceptor properties of substituents on the threemembered ring. ${ }^{[9]}$ Despite the groundbreaking works of Stork,
Danishefsky, Corey and co-workers during the 1970 's, ${ }^{[10]}$ intramolecular cyclizations involving activated cyclopropanes have been only rarely investigated. Until 2008, the formal homo-Nazarov cyclization of vinyl or aryl cyclopropyl ketones to form cyclohexenones was limited in scope and required superstoichiometric amounts of strong Lewis or Brønsted acids (Scheme 2). ${ }^{[7 \mathrm{a}-\mathrm{e}]}$ In 1980, Murphy showed that an excess of $\mathrm{SnCl}_{4}$ allowed the cyclization of aryl-cyclopropyl-ketones for the synthesis of aryltetralones in good yields (Scheme 2, A).$^{[7 a-c]}$ In 1988, Tsuge reported the first cyclization of vinyl-cyclopropyl ketones using an excess of polyphosphoric acid at $80^{\circ} \mathrm{C}$ (Scheme 2, B). ${ }^{[7 \mathrm{~d}]}$ The reaction was not general and several by-products were obtained. In 2008, Yadav and coworkers reported the cyclization of silylmethylcyclopropyl-ketones using 3 equivalents of $\mathrm{SnCl}_{4}$ at $80^{\circ}$ $\mathrm{C}$ (Scheme 2, C).${ }^{[7 \mathrm{e}]}$ In this work, the scope of the reaction could be extended to a broad range of heterocyclic systems, but the utility of the method was limited by the harsh conditions needed.
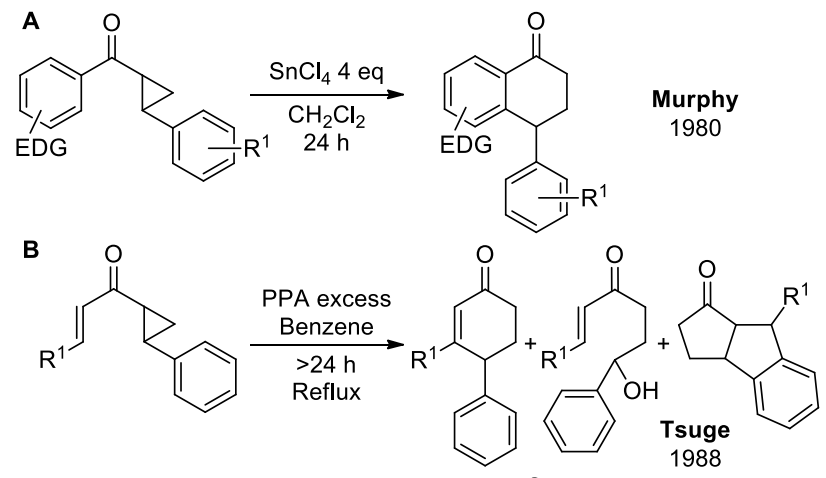<smiles>[R5]C[C@H]1C[C@@H]1C(=O)C1=CCCCCC1</smiles>

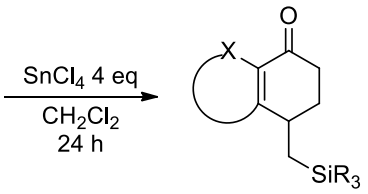

Yadav 2008

Scheme 2. Early examples of formal homo-Nazarov cyclizations.

Inspired by the progress in the Nazarov reaction, we decided to develop the first catalytic formal homo-Nazarov cyclization. We speculated that activation via adequate polarization of the cyclopropane ring could also lead to milder catalytic conditions. In our first studies we examined the effect of an electron-donating aromatic group in $\beta$ position to the carbonyl on the cyclopropane in combination with an enol ether, an electron-rich aromatic ring or an allyl silane as a nucleophilic double bond (Scheme 3). ${ }^{[7 f]}$ Using a catalytic amount of $p$-toluenesulfonic acid in polar solvents, we were able to access the desired cyclohexenones under mild conditions. The reaction tolerated variation of the size and nature of the groups attached to the double bond and worked with several electron-rich aromatic groups, but no reaction was observed with a simple phenyl group. Furthermore, no asymmetric induction could be obtained when using chiral Brønsted or Lewis acids.

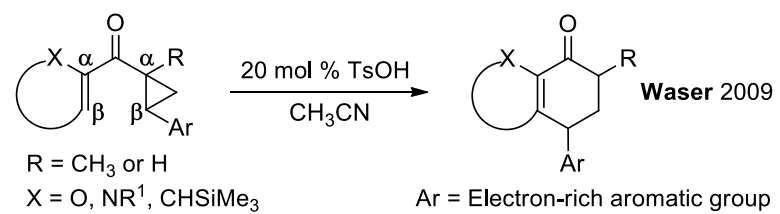

Scheme 3. Catalytic formal homo-Nazarov cyclization. 
In order to overcome these limitations, we decided to apply two strategies (Figure 1): (1) The introduction of an ester group on the cyclopropane at the $\alpha$ position to the ketone. The electronwithdrawing ester group was expected to increase the polarization of the cyclopropane. We examined this approach for the first time in 2009. ${ }^{[11]}$ In 2010, France and co-workers reported the use of the same class of substrates using indium catalysts, which allowed them to extend significantly the scope of the reaction. ${ }^{[7 \mathrm{~h}-\mathrm{i}]}$ Apart for enhancing the reactivity, we also thought that this class of substrates will offer unique opportunity for asymmetric induction, as twopoints binding with chiral Lewis acids will become possible. (2) The substitution of the aromatic group on the cyclopropane by a heteroatom. The lone pair of the heteroatom would give the electron density required for ring-opening under mild conditions. As heteroatoms are omnipresent in bioactive compounds, the obtained products would furthermore be highly useful for synthetic applications.

Herein we present first our preliminary results on the former strategy, the synthesis and cyclization of $\beta$-ketoester cyclopropanes and the first study of this class of substrates in asymmetric cyclizations. Concerning the latter approach, we have reported in 2010 the first example of the formal homo-Nazarov cyclization of aminocyclopropanes and their application in the synthesis of natural alkaloids. ${ }^{[7 \mathrm{~g}]}$ We now present the details of the challenging synthesis of the aminocyclopropanes, the full study of the scope of their cyclization, in particular using ketones derived from electron-rich heterocycles, as well as a comparison with an oxycyclopropane. We also discuss more in details the mechanism of the cyclization reaction based on experimental and computational data.

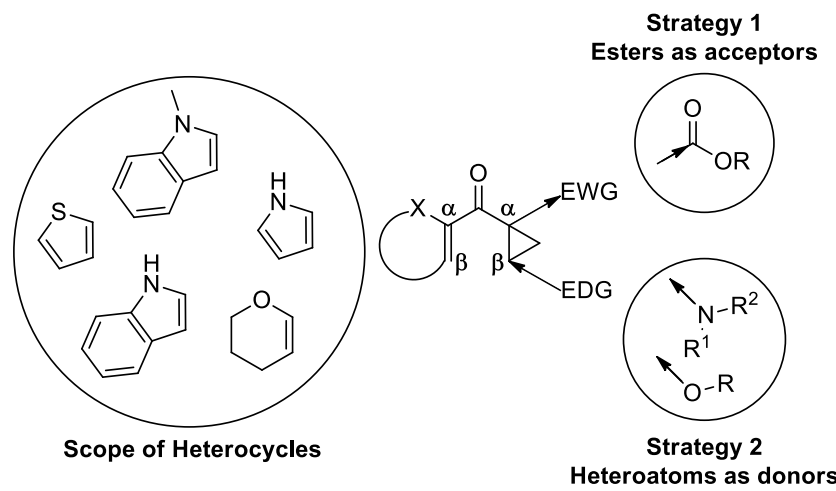

Figure 1. Cyclopropane polarization and heterocycles scope in the formal homoNazarov reaction.

\section{Results and Discussion}

\section{Activation with an Ester Substituent.}

The use of $\beta$-ketoester cyclopropanes was expected to lead to more stable enolate intermediates and consequently to allow milder reaction conditions. $\beta$-Ketoesters are also known to favor two pointbinding interactions with chiral Lewis acid catalysts allowing an easier control of the enantioselectivity. In this context, Trauner ${ }^{[5-c]}$ proposed in 2004 one of the first examples of a catalytic Nazarov reaction using a scandium PYBOX complex with ketoesters. Aggarwal $^{[5 \mathrm{a}]}$ and Togni ${ }^{[5 \mathrm{~d}]}$ also reported examples of asymmetric induction for similar substrates using chiral copper and nickel catalysts respectively. More recently, Johnson and co-workers have studied extensively Lewis acid catalyzed [3+2] annulations of polarized 1,1-diester cyclopropanes with aldehydes and they also reported an asymmetric cycloaddition using chiral $\mathrm{Mg}$ PYBOX catalysts. $^{[12]}$

Starting from dimethyl malonate ester (1), we first developed a synthetic route towards cyclopropyl $\beta$-ketoesters $6 \mathbf{b}-\mathbf{f}$ (Scheme 4). The synthesis of cyclopropane $\mathbf{6 a}$ has already been described in our previous work. ${ }^{[7]}$ A Regitz diazo transfer, ${ }^{[13]}$ followed by Rhcatalyzed cyclopropanation of the required olefins afforded the desired 1,2 cyclopropane diesters $3 \mathbf{b}-\mathbf{f}^{[14]}$ In a first approach, the addition of lithiated dihydropyran $\mathbf{5}$ on diesters $3 \mathbf{b}$ gave $\mathbf{6 b}$ as a diasteromeric mixture in low yield and with the undesired formation of double addition products. Therefore a more selective synthetic strategy was developed. Hydrolyzing the less sterically hindered of the two esters would give a carboxylic acid, which could be deprotonated and form a stable lithium complex. This will serve two roles: deactivating the acid to solve the regioselectivity problem and stabilize the tetrahedral intermediate obtained after addition of an organometallic reagent in order to prevent double addition. In the event, a selective saponification of the less sterically hindered trans ester group in $\mathbf{3 c - f}$ led to the corresponding acids $\mathbf{4 c - f}$ in high yields without further purification. ${ }^{[15]}$ Gratifyingly, treating carboxylic acids $\mathbf{4 c - f}$ with two equivalents of lithiated dihydropyran $\mathbf{5}$ led to regioselective formation of the corresponding keto acids in high yields. Finally, methylation of the free carboxylate gave access to the desired substrates $\mathbf{6 c}-\mathbf{f}$. Since this strategy afforded the addition products on the more hindered face of the dicarbonyl compounds, it constitutes a new method to obtain selectively the more sterically hindered trans-cyclopropane ketoesters.
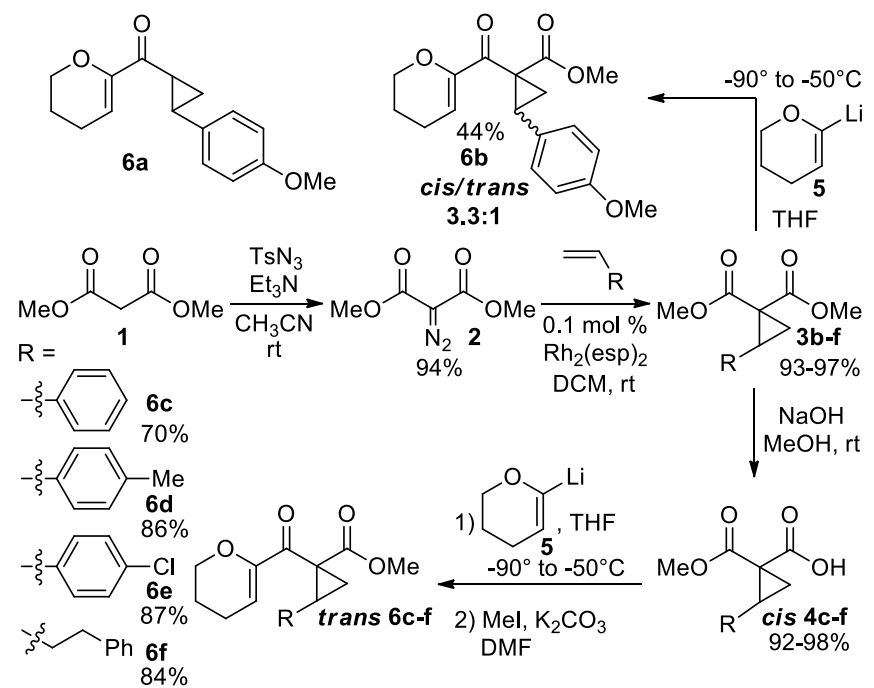

Scheme 4. Synthesis of cyclopropane ketoesters 6a-f.

In order to have an impression on the reactivity of the ketoester substrates, the reaction in presence of several Brønsted and Lewis acids was first monitored qualitatively by ${ }^{1} \mathrm{H}$ NMR spectroscopy (Table 1). $\beta$-Ketoester cyclopropane $\mathbf{6 b}$ was submitted to the catalytic conditions used in our previous work (entry 2). ${ }^{[7]}$ Gratifyingly, it displayed an enhanced reactivity when compared with ketone cyclopropane $\mathbf{6 a}$ using the standard condition for the formal homo-Nazarov cyclization $(20 \mathrm{~mol} \%$-toluenesulfonic acid in $\mathrm{CH}_{3} \mathrm{CN}$ ): With $\beta$-ketoesters $6 \mathbf{b}$ complete conversion was observed after only 15 minutes at rt (entry 2), whereas 18 hours were required in absence of the ester group (entry 1). Since phenyl- 
substituted cyclopropane 6c gave no conversion in our previous work, ${ }^{[7 f]}$ we decided then to study the activating effect of the ester group for this substrate. However, no conversion was observed with this substrate using $p$-toluenesulfonic acid as catalyst (entry 3). Increasing the strength of the Brønsted acid was unsuccessful (entries 4 and 5). $\mathrm{Cu}(\mathrm{OTf})_{2}, \mathrm{Sn}(\mathrm{OTf})_{2}$, and $\mathrm{MgI}_{2}$ showed a very slow conversion rate at $\mathrm{rt}$ while inducing partial decomposition when heated (entries 6-11). On the other hand, stronger Lewis acids such as $\mathrm{SnCl}_{4}$ or $\mathrm{AlCl}_{3}$ gave extensive decomposition in less than one hour (entries 12 and 13). In the search for a milder catalyst, we tested $\mathrm{Ni}\left(\mathrm{ClO}_{4}\right)_{2} \bullet 6 \mathrm{H}_{2} \mathrm{O}$, which had proven to be an effective Lewis acid for the opening of donor-acceptor cyclopropanes, both as anhydrous ${ }^{[12 a, 16]}$ or as aquo complex. ${ }^{[15]}$ In our hand, only the aquo complex was active and gave a clean and complete conversion of $\mathbf{6 c}$ after $26 \mathrm{~h}$, with $63 \%$ isolated yield of 7c (entry 14). Surprisingly $\mathrm{BF}_{3} \cdot \mathrm{Et}_{2} \mathrm{O}$, which gave decomposition with $\mathbf{6 a}$ (entry 15), was the catalyst of choice for $\beta$-ketoesters cyclopropane $\mathbf{6 c}$ (entry 16), promoting the cyclization in $83 \%$ yield and a short reaction time.

Table 1. Preliminary screening of conditions for the homo-Nazarov cyclization for ketoester substrates.
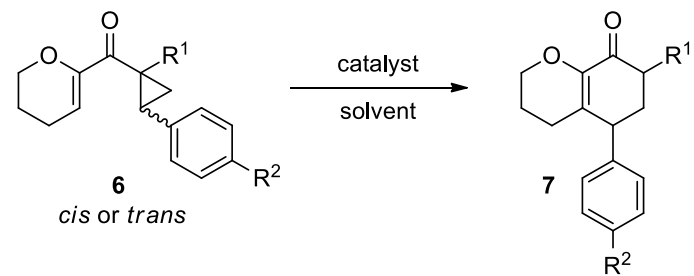

\begin{tabular}{|c|c|c|c|c|c|c|}
\hline Entry $^{[a]}$ & $\mathrm{R}^{1}$ & $\mathrm{R}^{2}$ & Catalyst (mol \%) & Solvent & Time & Conversion $^{[\mathrm{b}]}$ \\
\hline 1 & $\mathrm{H}$ & $\mathrm{MeO}(\mathbf{6 a})$ & $\mathrm{TsOH}(20)$ & $\mathrm{CH}_{3} \mathrm{CN}$ & $18 \mathrm{~h}$ & $100 \%$ \\
\hline 2 & $\mathrm{CO}_{2} \mathrm{Me}$ & $\mathrm{MeO}(\mathbf{6 b})$ & $\mathrm{TsOH}(20)$ & $\mathrm{CH}_{3} \mathrm{CN}$ & $15 \mathrm{~min}$ & $100 \%$ \\
\hline 3 & $\mathrm{CO}_{2} \mathrm{Me}$ & $\mathrm{H}(\mathbf{6 c})$ & $\mathrm{TsOH}(20)$ & $\mathrm{CH}_{3} \mathrm{CN}$ & $19 \mathrm{~h}$ & $0 \%$ \\
\hline 4 & $\mathrm{CO}_{2} \mathrm{Me}$ & $\mathrm{H}(\mathbf{6} \mathbf{c})$ & TfOH (5) & $\mathrm{CH}_{2} \mathrm{Cl}_{2}$ & $19 \mathrm{~h}$ & $0 \%$ \\
\hline 5 & $\mathrm{CO}_{2} \mathrm{Me}$ & $\mathrm{H}(\mathbf{6} \mathbf{c})$ & $\mathrm{H}_{2} \mathrm{SO}_{4}(5)$ & $\mathrm{CH}_{2} \mathrm{Cl}_{2}$ & $19 \mathrm{~h}$ & $0 \%$ \\
\hline 6 & $\mathrm{CO}_{2} \mathrm{Me}$ & $\mathrm{H}(\mathbf{6} \mathbf{c})$ & $\mathrm{Cu}(\mathrm{OTf})_{2}(20)$ & $\mathrm{CH}_{2} \mathrm{Cl}_{2}$ & $36 \mathrm{~h}$ & $<20 \%$ \\
\hline 7 & $\mathrm{CO}_{2} \mathrm{Me}$ & $\mathrm{H}(\mathbf{6} \mathbf{c})$ & $\mathrm{Sn}(\mathrm{OTf})_{2}(15)$ & $\mathrm{CH}_{2} \mathrm{Cl}_{2}$ & $26 \mathrm{~h}$ & $<20 \%$ \\
\hline 8 & $\mathrm{CO}_{2} \mathrm{Me}$ & $\mathrm{H}(\mathbf{6} \mathbf{c})$ & $\mathrm{MgI}_{2}(50)$ & $\mathrm{CH}_{2} \mathrm{Cl}_{2}$ & $18 \mathrm{~h}$ & $<20 \%$ \\
\hline 9 & $\mathrm{CO}_{2} \mathrm{Me}$ & $\mathrm{H}(\mathbf{6} \mathbf{c})$ & $\mathrm{Cu}(\mathrm{OTf})_{2}(15)$ & $\mathrm{CH}_{2} \mathrm{Cl}_{2}$ & $20 \mathrm{~min}$ & $\operatorname{dec}^{[\mathrm{c}],[\mathrm{d}]}$ \\
\hline 10 & $\mathrm{CO}_{2} \mathrm{Me}$ & $\mathrm{H}(\mathbf{6 c})$ & $\mathrm{Sn}(\mathrm{OTf})_{2}(5)$ & DCE & $15 \mathrm{~min}$ & $\operatorname{dec}^{[\mathrm{cc},[\mathrm{e}]}$ \\
\hline 11 & $\mathrm{CO}_{2} \mathrm{Me}$ & $\mathrm{H}(\mathbf{6} \mathbf{c})$ & $\mathrm{MgI}_{2}(15)$ & DCE & $1.15 \mathrm{~h}$ & $\operatorname{dec} .[\mathrm{cc},[\mathrm{e}]$ \\
\hline 12 & $\mathrm{CO}_{2} \mathrm{Me}$ & $\mathrm{H}(\mathbf{6} \mathbf{c})$ & $\mathrm{SnCl}_{4}(50)$ & $\mathrm{CH}_{2} \mathrm{Cl}_{2}$ & $2.3 \mathrm{~h}$ & dec..$^{[c]}$ \\
\hline 13 & $\mathrm{CO}_{2} \mathrm{Me}$ & $\mathrm{H}(\mathbf{6} \mathbf{c})$ & $\mathrm{AlCl}_{3}(50)$ & $\mathrm{CH}_{2} \mathrm{Cl}_{2}$ & $1 \mathrm{~h}$ & dec..$^{[c]}$ \\
\hline 14 & $\mathrm{CO}_{2} \mathrm{Me}$ & $\mathrm{H}(\mathbf{6 c})$ & $\mathrm{Ni}\left(\mathrm{ClO}_{4}\right)_{2} \bullet 6 \mathrm{H}_{2} \mathrm{O}(20)$ & $\mathrm{CH}_{2} \mathrm{Cl}_{2}$ & $26 \mathrm{~h}$ & $100 \%(63 \%)^{[\mathrm{f]}}$ \\
\hline 15 & $\mathrm{H}$ & $\mathrm{MeO}(\mathbf{6 a})$ & $\mathrm{BF}_{3} \cdot \mathrm{Et}_{2} \mathrm{O}(50)$ & $\mathrm{CH}_{2} \mathrm{Cl}_{2}$ & $25 \mathrm{~min}$ & dec. $^{[\mathrm{cc}]}$ \\
\hline 16 & $\mathrm{CO}_{2} \mathrm{Me}$ & $\mathrm{H}(\mathbf{6 c})$ & $\mathrm{BF}_{3} \cdot \mathrm{Et}_{2} \mathrm{O}(10)$ & $\mathrm{CH}_{2} \mathrm{Cl}_{2}$ & $3 \mathrm{~h}$ & $100 \%(83 \%)^{[\mathrm{f]}}$ \\
\hline
\end{tabular}

[a] 6a and 6c only trans, $\mathbf{6 b}$ as a mixture cis/trans 3.3:1. [b] Reaction run with 50-400 $\mu \mathrm{mol} 6$ in $0.5-4 \mathrm{~mL}$ solvent. Conversion estimated by comparison of integration of peaks of 6 and 7 in the ${ }^{1} \mathrm{H}$ NMR spectra of the crude reaction mixture. See supporting information for details. [c] Decomposition of the starting material was observed. [d] Reaction run at $110{ }^{\circ} \mathrm{C}$. [e] Reaction run at $55^{\circ} \mathrm{C}$. [f] Isolated yield after column chromatography.

With the optimized conditions in hand, we also examined methyl and chloro substituents on the benzene ring (Scheme 5). Fortunately, the additional reactivity given by the $\beta$-ketoesters could compensate even the electron-deficiency of the $p$-chlorophenyl group in 6e. In order to facilitate characterization, the cyclic compounds were decarboxylated in the presence of $\mathrm{NaCl}$ in wet DMSO. In a further experiment, no conversion was observed when the aromatic group was replaced with a 2-phenylethyl substituent in 6f. This last result underlined the importance of the stabilizing effect of the aromatic group for the reactivity of the cyclopropane.

We also detected a progressive epimerization of the cyclopropane during the homo-Nazarov reaction. Using either cis or trans phenylcyclopropane $\mathbf{6 c}$, we isolated the same mixture of syn and anti cyclic isomers with 1:1.5 diastereoselectivity. This result would be expected if the reaction proceeded via a carbocation or if equilibration of the products was occurring under these conditions.

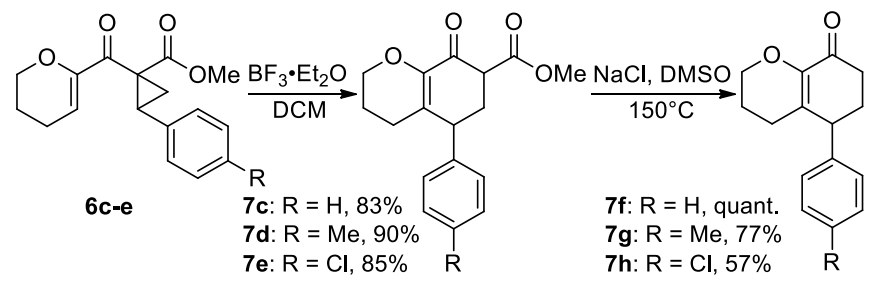

Scheme 5. Effect of the substitution on the aromatic ring on the cyclization reaction on preparative scale.

Inspired by the recent works in asymmetric cyclization with chiral Lewis acids of Johnson, ${ }^{[12]}$ Trauner ${ }^{[5 \mathrm{~b}-\mathrm{c}]}$ and Togni, ${ }^{[5 \mathrm{~d}]}$ we then turned to the development of an asymmetric version of the homoNazarov cyclization. Preliminary studies were realized with substrate $\mathbf{6 b}$ and $\mathbf{6 g}$, since the $p$-methoxyphenyl group allowed a rapid ring opening of the cyclopropane, which had been shown to be crucial for the dynamic kinetic asymmetric transformation of racemic cyclopropanes. ${ }^{[12 b]}$ The more sterically hindered tert-butyl ester $\mathbf{6 g}$ was prepared by addition of lithiated pyran $\mathbf{5}$ to the corresponding tert-butyl-methyl cyclopropane diester. ${ }^{[17]}$ Both methyl $(\mathbf{6 b})$ and tert-butyl $(\mathbf{6 g})$ esters were then tested in the asymmetric reaction in order to analyze the steric influence of the ester on the stereoselectivity. Basing on the work of Johnson, ${ }^{[12]}$ PYBOX ligands 8 and $\mathbf{9}$ were tested in association with $\mathrm{Mg}$ salts (Scheme 6). As the mixture of diastereoisomers formed during the homo-Nazarov reaction made analysis difficult, the $e e$ values were measured on the decarboxylated product 7a. Only $11 \%$ ee was observed when using the methyl ester $\mathbf{6 b}$. A slightly higher value $(25 \%)$ could be achieved using tert-butyl ester 6g. Although the observed enantioselectivity is still very low, these preliminary results constituted the first example of asymmetric induction in the formal homo-Nazarov reaction. Further screening of chiral catalysts, especially those successful in the Nazarov reaction, ${ }^{[5]}$ in order to increase the selectivity of the reaction will be part of future work.

\section{Introduction of Heteroatoms on the Cyclopropane.}

Introduction and Substrates Synthesis: Although new conditions with ketoesters were successfully developed, the necessary presence of an aromatic stabilizing group still constituted a strong limitation in terms of application and scope. Replacing the aromatic group by a heteroatom would give a good stabilization of the formed carbocation and at the same time allow broadening the range of structures accessible. For example, a nitrogen- substituted cyclopropane would generate a zwitterionic species stabilized as an acyliminium intermediate, which could also be considered as the product of a retro-Mannich reaction, a process difficult in the case of acyliminium ions. Moreover, the cyclohexylamines obtained from the aminocyclopropanes are largely represented in the core structure 
of several natural alkaloids such as aspidospermidine (10), strychnine (11), vinblastine (12) and vincristine (13) (Figure 2).

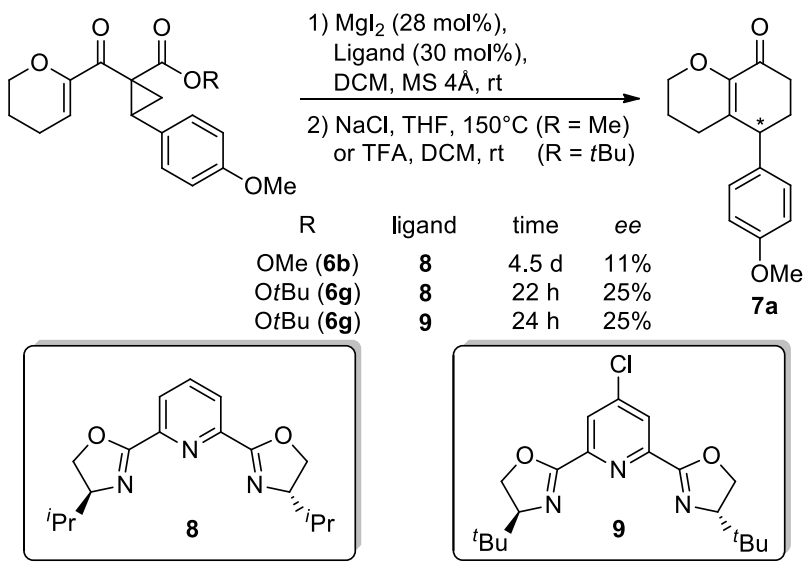

Scheme 6. Attempts towards an asymmetric homo-Nazarov cyclization.
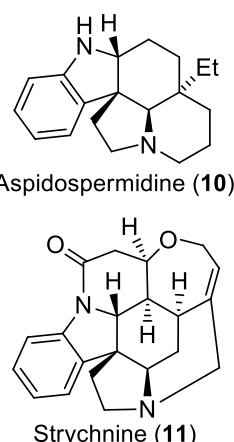

Strychnine (11)
Aspidospermidine (10)

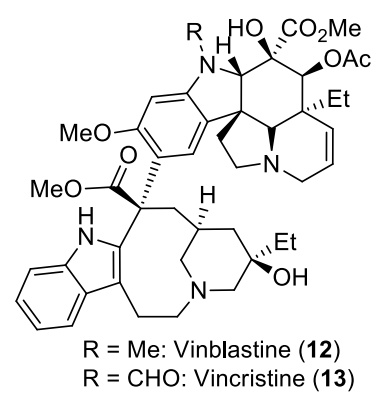

Figure 2. Examples of alkaloid natural products containing a cyclohexylamine ring.

Despite the importance of acyliminium cyclization in alkaloid synthesis, there are only few examples on the use of aminocyclopropanes to generate these intermediates. In the seminal work of Wenkert, aminocyclopropanes were opened under acidic conditions to generate the corresponding lactones, which were used as precursors of acyliminium intermediates in intermolecular addition reactions on indole derivatives. ${ }^{[18]}$ To the best of our knowledge, the only example of intramolecular cyclization of aminocyclopropanes has been reported by Six and co-workers, who studied the intramolecular electrophilic aromatic substitution of simple alkyl cyclopropanes. ${ }^{[19]}$

Oxycyclopropanes on the other hand were shown to undergo formal homo-Nazarov cyclization in presence of stoichiometric amount of a Lewis acid. ${ }^{[7]}$ Since the electron-density of oxycyclopropanes could be compared with that of aminocyclopropanes substituted with a carbamate group, similar reactivity in the catalytic formal homo-Nazarov cyclization could be expected. We speculated that the high electron-density of both amino- and oxy- cyclopropanes should allow their use also under catalytic conditions. We consequently decided to examine a range of amino- and oxy- cyclopropanes to assess the potential of the reaction (Figure 3).
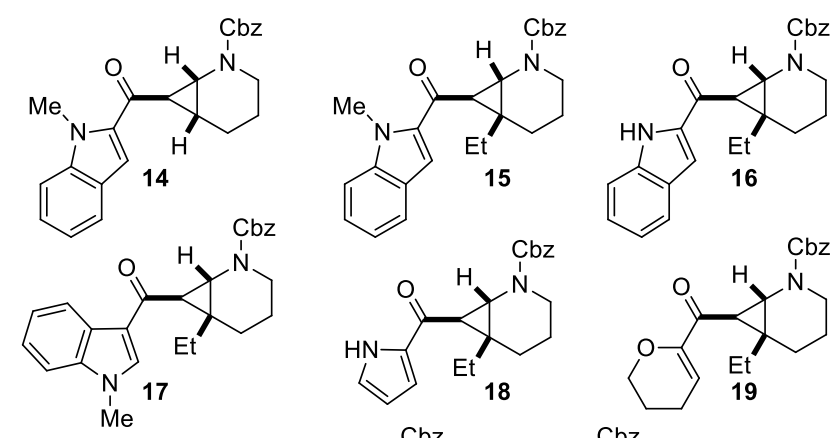

$\mathrm{Cbz}$

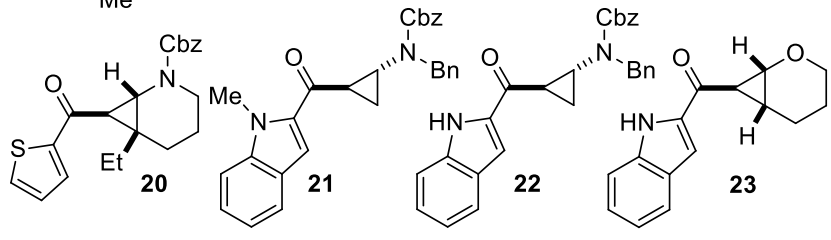

Figure 3. Substrates synthesized for the formal homo-Nazarov cyclization.

As a first attempt, we decided to examine substrate $\mathbf{1 4}$, as it would lead to the tetracyclic scaffold of the aspidosperma alkaloid aspidospermidine (10). The synthesis of $\mathbf{1 4}$ started with $\delta$ valerolactam (24), which was protected, reduced and dehydrated to afford enamide 26 (Scheme 7). ${ }^{[20]}$ The non-optimized conditions of cyclopropanation using copper-bronze gave a diastereomeric mixture of esters 27. A sequence of hydrolysis and amide coupling yielded Weinreb amide 29. In the hydrolysis step, only the less sterically hindered exo-cyclopropane reacted, allowing the isolation of a single isomer of acid $\mathbf{2 8}$.

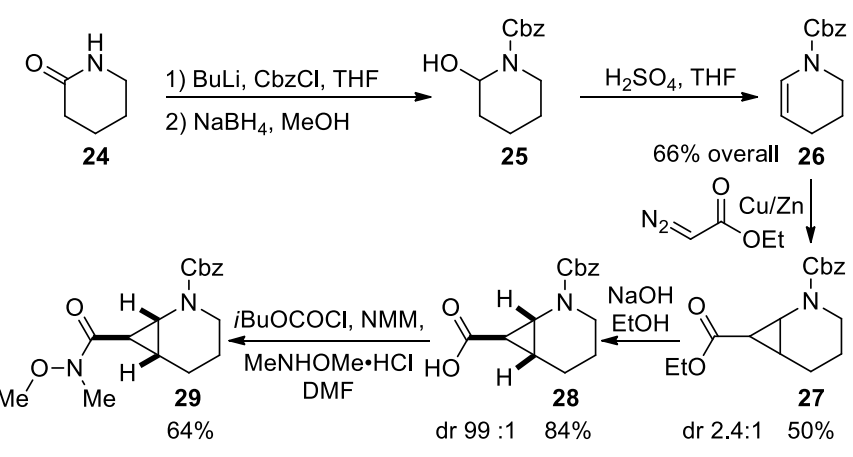

Scheme 7. Synthesis of Weinreb amide 29.

Optimization of the coupling involving 2-lithiated- $N$-methyl indole (30) with the amide $\mathbf{2 9}$ was required, as we only observed the formation of enamide $\mathbf{3 1}$ derived from cyclopropane ring opening under standard conditions (Scheme 8). We speculated that cyclopropane opening was favored due to the high stability of the $\mathrm{N}, \mathrm{O}$-lithium chelate formed upon addition of the organometallic reagent. The addition of potassium tert-butoxide could potentially lead to the exchange of lithium by potassium and form a weaker chelate, which should favor a faster elimination of the hydroxylamine and preserve the cyclopropane integrity. Indeed, we were pleased to obtain the desired product $\mathbf{1 4}$ in $67 \%$ yield using this approach. 


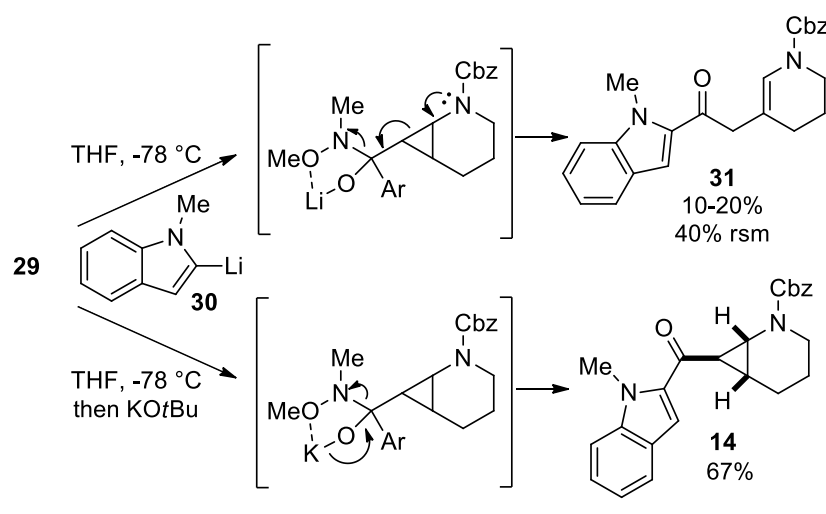

Scheme 8. Coupling of Weinreb amide $\mathbf{2 9}$ with lithiated indole $\mathbf{3 0 .}$

Using the standard conditions developed for the catalytic homoNazarov cyclization, we were delighted to isolate the cyclic compound 32 in $90 \%$ yield with high diasteroselectivity for the cisfused product (Scheme 9). As NMR analysis of $\mathbf{3 2}$ was difficult due to the presence of carbamate rotamers, it was deprotected prior to complete structure assignment.

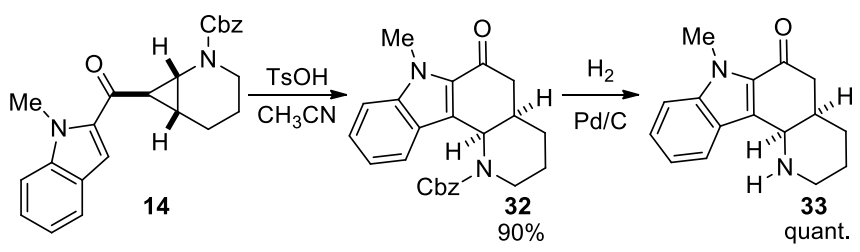

Scheme 9. Cyclization of indole aminocyclopropane $\mathbf{1 4}$

With this successful result in hand we decided to synthesize the ethyl-substituted cyclopropane $\mathbf{1 5}$, as the ethyl group is present in aspidospermidine (10) (Scheme 10).
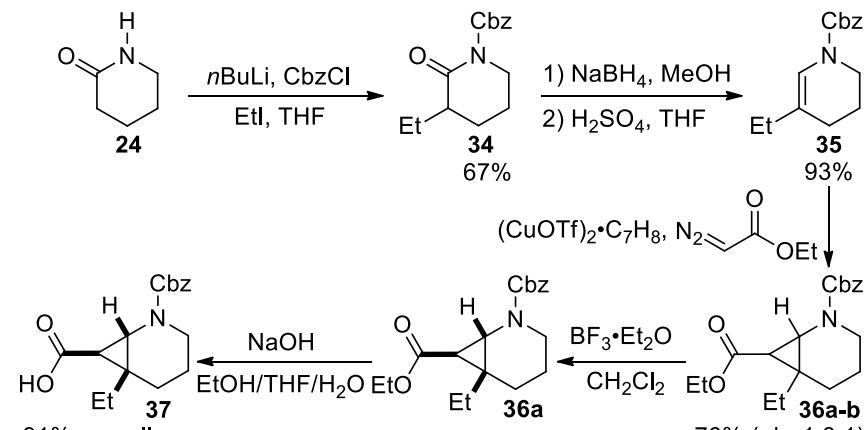

$91 \%$ overall

$76 \%(\mathrm{dr}: 1.3: 1)$

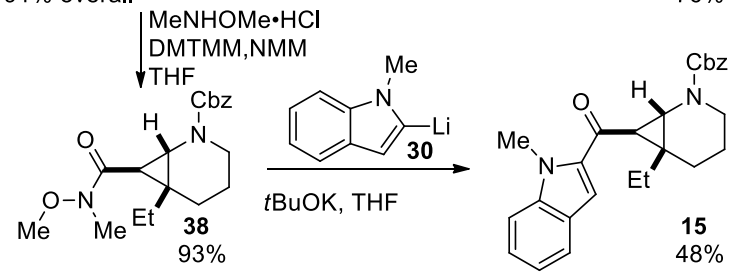

Scheme 10. Synthesis of ethyl-substituted cyclopropane $\mathbf{1 5}$

Following a modified procedure of Grieco, ${ }^{[20]} \delta$-valerolactam (24) was alkylated and protected in one pot. Lactam 34 was then reduced and the resulting half aminal dehydrated to afford enamide 35. The fine tuning of catalyst and reaction time was crucial for the reproducibility and the yield of the cyclopropanation of $\mathbf{3 5}$. Since the use of $\mathrm{Cu} / \mathrm{Zn}$ alloy in the cyclopropanation gave no reproducible results, we decided to test several copper and rhodium catalysts. The slow addition of a diluted solution of ethyl diazoacetate was important to avoid extensive polymerization and copper (I) catalysts allowed a better yield when compared to rhodium(II) or copper (II) catalysts. Using copper (I) triflate and syringe-pump addition of an ethyl diazoacetate solution, a diasteromeric mixture $(1.3: 1 \mathrm{dr})$ of cyclopropane ethyl esters 36a and 36b was obtained in a reproducible $76 \%$ of yield. The isomerization of the endo ester $\mathbf{3 6} \mathbf{b}$ into the more stable exo form 36a was possible with catalytic $\mathrm{BF}_{3} \cdot \mathrm{OEt}_{2}$ in excellent yield. ${ }^{[20]}$ Ester 36a was then saponified affording the carboxylic acid $\mathbf{3 7}$ as a pure diasteroisomer. The use of 4-(4,6-dimethoxy-1,3,5-triazin-2-yl)-4-methylmorpholinium chloride (DMTMM) allowed the formation of Weinreb amide $\mathbf{3 8}$ in good yield without further purification. ${ }^{[21]}$ The coupling reaction between 2-lithiated- $N$-methylindole (30) and Weinreb amide 38 afforded the precursor $\mathbf{1 5}$ of the homo-Nazarov reaction.

As aspidodermidine contains a free indole nitrogen, cyclization of substrate $\mathbf{1 6}$ with an unprotected indole would be more efficient. When using a free indole in the cyclization however, regioselectivity could become a serious issue, as cyclization on the nitrogen could also occur. For this reason cyclopropane 39, bearing a free indole was first synthesized starting from lithiated $N$-carboxy indole and the corresponding Weinreb amide cyclopropane. We were delighted to find that a catalytic amount of $p$-toluenesulfonic acid in acetonitrile gave exclusive $\mathrm{C} 3$ cyclization product $\mathbf{4 0}$ in quantitative yield (Scheme 11).

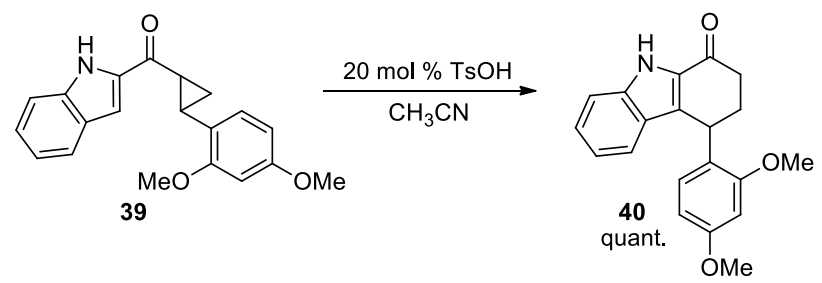

Scheme 11. Cyclization of free indole cyclopropane 39

With these promising preliminary results in hand, we move on to the synthesis of indole 16. The use of indole $N$-carboxylates pioneered by Katritsky ${ }^{[22]}$ presented several advantages for our synthetic strategy: The directing group property of carboxylate favored the selective lithiation on the $\mathrm{C}-2$ position of indole and a protecting group free 2-carbonyl indole is generated after aqueous work up. Nevertheless, this method had been used only with simple substrates in the past. The lithium indole- $N$-carboxylate was initially prepared in situ via a sequence of deprotonation/carboxylation/lithiation then added at $-78^{\circ} \mathrm{C}$ into a solution of Weinreb amide $\mathbf{3 8}$ affording $\mathbf{1 6}$ in low yield. Optimization of additives, temperature, stoichiometry and reaction time and the use of pre-formed and recrystallized indole- $N$ carboxylic acid $\mathbf{4 1}$ resulted in an increased yield and reproducible results on a multi-grams scale (Scheme 12). In particular, the use of $\mathrm{LiCl}$ as additive and the slow increase of temperature from $-78^{\circ} \mathrm{C}$ to $-20^{\circ} \mathrm{C}$ combined with a reversed quench at $0^{\circ} \mathrm{C}$ were crucial to prevent side reactions. This implementation of Katritsky method in 
complex settings allowed a highly convergent synthesis of indole cyclopropane $\mathbf{1 6}$.

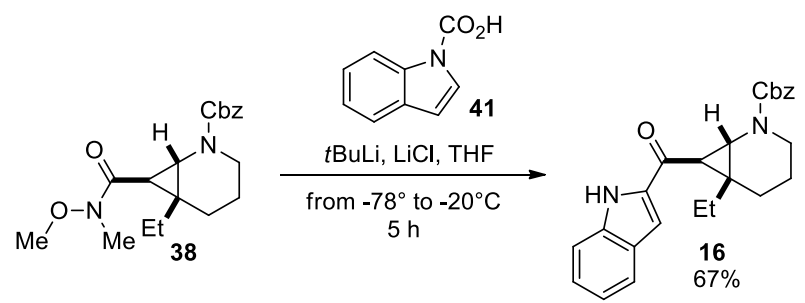

Scheme 12. Synthesis of indole cyclopropane $\mathbf{1 6}$

In our previous publication we tested successfully the cyclization involving substituted indoles. ${ }^{[7 \mathrm{~g}]}$ Encouraged by these promising results, a further series of substrates was synthesized in order to obtain more information regarding the scope of heterocycles in the cyclization step. For this purpose, a range of heterocycles with decreasing nucleophilicity were targeted as nucleophiles in the cyclization reaction. We focused our studies on the cyclopropanes derived from Weinreb amide 38 since the cyclization products would represent interesting analogues of natural alkaloids. Combining the appropriate metallated heterocycle with Weinreb amide 38, we generated 3-indole-, pyrrole-, dihydropyran- and thiophene- substituted carbonylcyclopropanes 17-20 (Scheme 13). The pyrrole was carboxylated on its free nitrogen prior to lithiation. For these new substrates, no attempt was done to optimize the reaction conditions.

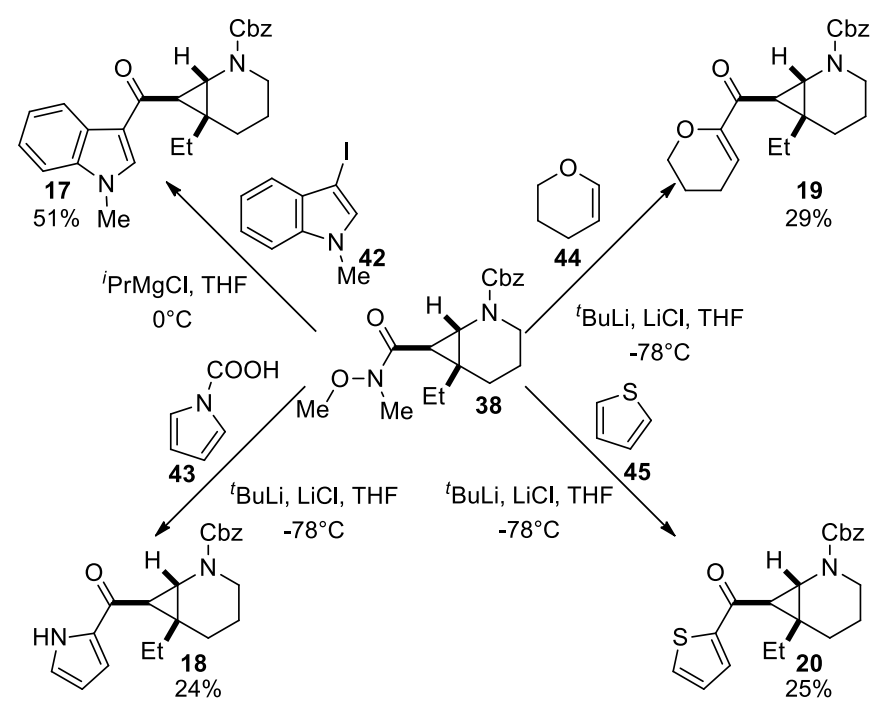

Scheme 13. Coupling of $\mathbf{3 8}$ with different heterocycles.

In order to further examine the scope and limitations of the aminocyclopropane cyclization, acyclic carbamates $\mathbf{2 1}$ and $\mathbf{2 2}$ were then synthesized (Scheme 14). The absence of rigidifying ring makes this class of compounds particularly challenging with regard to the cyclization step. The required enamide $\mathbf{4 6}$ was obtained from a Curtius rearrangement of acryloyl chloride followed by benzylation. ${ }^{[23]}$ The optimized cyclopropanation conditions gave a diastereoisomeric mixture ( $\mathrm{dr}$ 1:1) of aminocyclopropyl ethyl esters 47a and $\mathbf{4 7 b}$ in $69 \%$ yield. After isomerization with $\mathrm{BF}_{3} \cdot \mathrm{OEt}_{2}$ and saponification, the resulting acid $\mathbf{4 8}$ was converted into Weinreb amide 49. The right choice of lithiating agent, the fine tuning of reaction conditions and the use of $N$-methylindole $\mathbf{5 0}$ or carboxy indole $\mathbf{4 1}$ was crucial to give useful yields of cyclopropanes $\mathbf{2 1}$ and 22. ${ }^{[24]}$

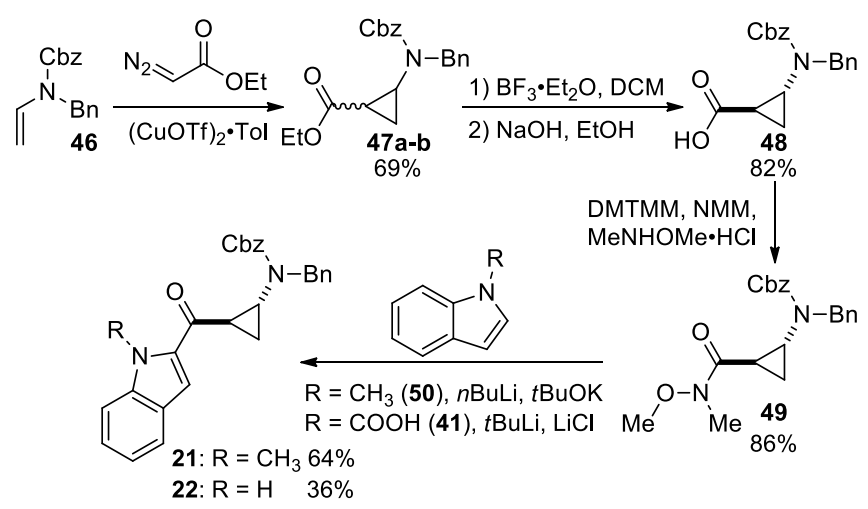

Scheme 14. Synthesis of acyclic cyclopropyl carbamates $\mathbf{2 1}$ and $\mathbf{2 2}$

We then synthesized the cyclic ether cyclopropane substrate $\mathbf{2 3}$ starting from dihydropyran (44) (Scheme 15). A high temperature cyclopropanation with copper catalyst and EDA afforded the desired ester $\mathbf{5 1}$ as a single diasteroisomer. ${ }^{[25]}$ Equilibration of the diastereoisomers probably did occur spontaneously through ring opening at high temperature. During saponification to afford the carboxylic acid, the oxycyclopropane ring partially opened, generating a vinylether side product 53. The mixture of open and closed forms was used directly in a peptide coupling with EDC affording an inseparable mixture of Weinreb amides $\mathbf{5 4}$ and $\mathbf{5 5}$. Finally, the separation of the two forms was possible after the reaction with the bis-lithiated carboxyindole obtained from $\mathbf{4 1}$ allowing the isolation of pure cyclopropane $\mathbf{2 3}$ (Scheme 15). ${ }^{[26]}$

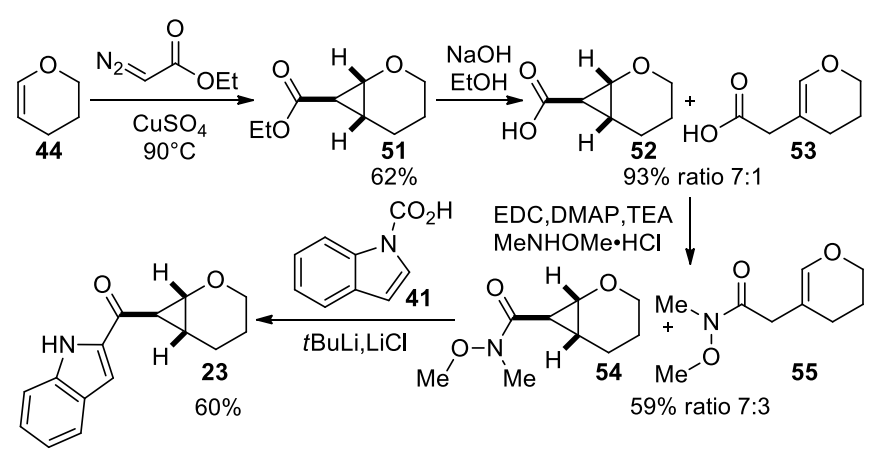

Scheme 15. Synthesis of oxycyclopropane $\mathbf{2 3}$.

Cyclization: Encouraged by the preliminary results obtained with indole 14 (Scheme 9), we examined the cyclization of ethyl substituted cyclopropane $\mathbf{1 5}$ required for the synthesis of aspidospermidine (10). The presence of a quaternary carbon and the resulting steric hindrance was expected to be a major challenge for the cyclization process. We were consequently delighted to observe quantitative cyclization with perfect diastereoselectivity under our standard conditions (Scheme 16). The high diastereoselectivity shown by the formal homo-Nazarov cyclization is an important advantage compared to other synthetic strategies proposed for the 
synthesis of aspidosperma alkaloids using aminocyclopropanes which were based on intermolecular addition and gave low stereoselectivity. ${ }^{[18 \mathrm{~d}]}$
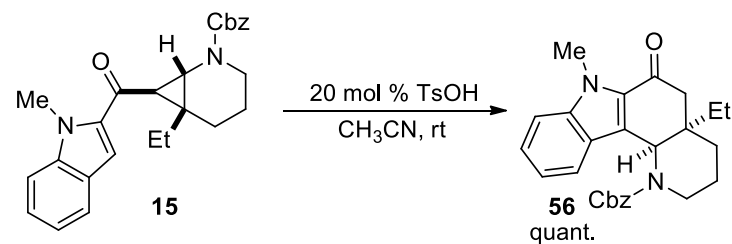

Scheme 16. Cyclization of ethyl-substituted cyclopropane $\mathbf{1 5}$.

In a first cyclization attempt for free indole 16, using the standard conditions for the homo-Nazarov cyclization, we were surprised to isolate two different compounds. After removal of the protecting group we were able to identify the desired product $\mathbf{5 7}$ obtained from the attack at the $\mathrm{C} 3$ position of indole and compound 58 derived from the cyclization on the $\mathrm{N} 1$ position in a 1.6:1 ratio (Scheme 17). A screening to find the right conditions to control the regioselectivity of the cyclization was carried out. The combination of polar solvent and a soft Lewis acid gave selectively the C-C cyclization compound while a strong Brønsted acid in an apolar solvent favored C-N bond formation (Scheme 17). ${ }^{[27]}$

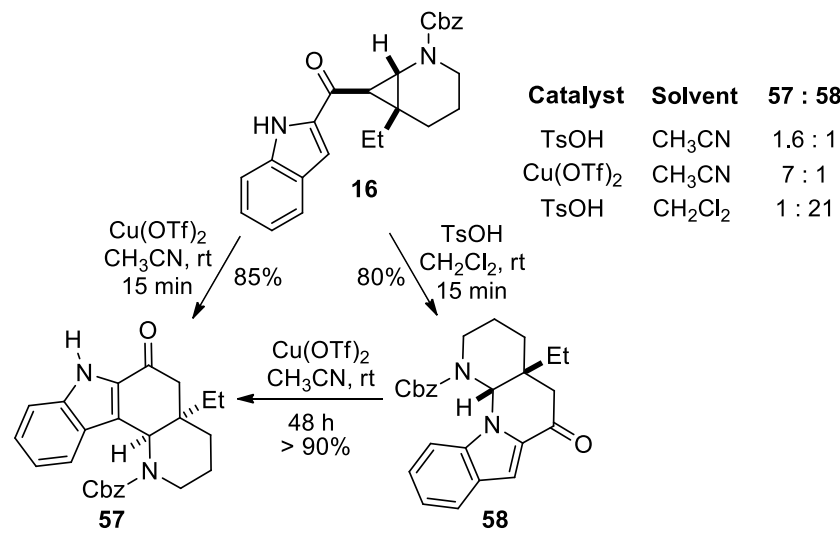

Scheme 17. Regio and diastero- selective cyclization of cyclopropane $\mathbf{1 6 .}$

One of the potential explanations we had proposed in our previous work $^{[7 \mathrm{~g}]}$ was based on the hard/soft reactivity of the generated charged intermediates (Scheme 18). The use of $\mathrm{Cu}(\mathrm{OTf})_{2}$ would generate a soft copper-bound enolate $\mathbf{I}$, which would promote the attack of the iminium on the softer $\mathrm{C} 3$ indole position with the higher electron-density in the HOMO orbital. Rearomatization of the cyclization product II and proton-transfer would lead to $\mathbf{5 7}$ and regenerate the catalyst. Moreover, the stabilizing effect offered by the polar solvent plays a fundamental role in favoring an orbital controlled over a charge-controlled cyclization. On the other hand, when the aminocyclopropane is activated with $p$-toluenesulfonic acid, the formed enol III would have a "hard" character, which would favor the attack of the formed acyl iminium at the harder N1 indole position. Using the non-coordinating solvent methylene chloride, a fast charge-controlled reaction on the harder nitrogen is further favored. From the obtained cyclization intermediate $\mathbf{I V}$, a simple deprotonation and tautomerization would give $\mathbf{5 8}$.

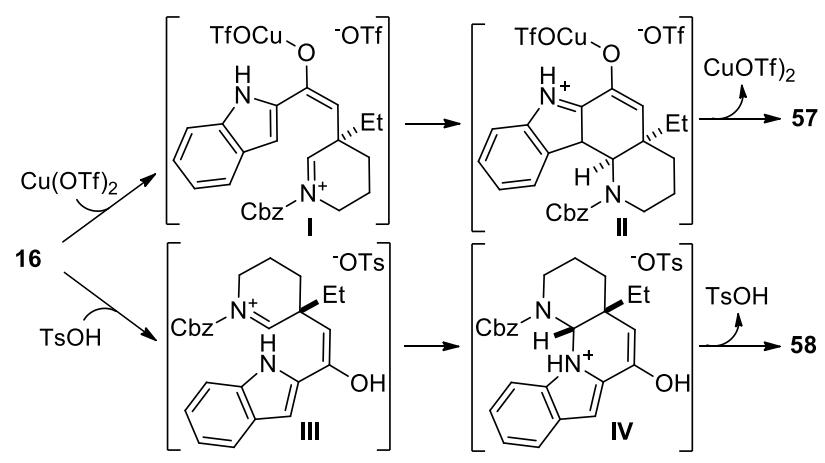

Scheme 18. Probable intermediates for the cyclization reactions.

The formation of the more stable thermodynamic product over the kinetic product should also be favored under the $\mathrm{Cu}$-catalyzed conditions. In order to support this hypothesis, we examined interconversion of the two cyclization products under the reaction conditions. It was possible to convert the cyclic compound $\mathbf{5 8}$ into the regioisomer $\mathbf{5 7}$ using copper triflate in acetonitrile (Scheme 17), whereas no interconversion was observed when $\mathbf{5 7}$ was submitted to p-toluenesulfonic acid in dichloromethane. These last results suggested that $\mathrm{C}-\mathrm{N}$ bond formation is under kinetic control, while $\mathrm{C}-\mathrm{C}$ cyclization is thermodynamically more favored. Nevertheless, the reaction using the $\mathrm{Cu}$ catalyst is probably leading directly to the C3 cyclization product, as the reaction time for the conversion of $\mathbf{5 8}$ into $\mathbf{5 7}$ was much longer than for the direct cyclization.

For a better understanding of the observed cyclization selectivity, geometry optimization calculations were performed on the four possible cyclization products (Figure 4), namely the cis and trans diastereomers arising from $\mathrm{C} 3$ cyclization (57 and 59 respectively), and the cis and trans diastereomers deriving from N1 cyclization (58 and 60 respectively). All geometry optimization calculations ${ }^{[28]}$ were performed at the DFT(B3LYP)/6-31+G* level in redundant internal coordinates $^{[29]}$ using the Gaussian 09 (G09) suite of programs. ${ }^{[30]}$ The nature of all found critical points (geometries are reported in the Supporting Information material) was assessed at the same level of theory and accuracy by means of frequency calculations. In all calculations the solvent (acetonitrile) effect was taken into account at the implicit level using the SCRF-CPM method (G09 defaults were used). ${ }^{[31]}$

The cis-fused ring systems $\mathbf{5 7}$ and $\mathbf{5 8}$ were found to be substantially lower in energy than their trans counterparts $\mathbf{5 9}\left(\Delta \mathrm{E}_{57}\right.$ $59=11.45 \mathrm{Kcal} / \mathrm{mol})$ and $\mathbf{6 0}\left(\Delta \mathrm{E}_{58-60}=9.97 \mathrm{Kcal} / \mathrm{mol}\right)$; this can be easily ascribed to the increased stability of the chair conformation adopted by the piperidine ring in compounds $\mathbf{5 7}$ and $\mathbf{5 8}$, compared to the less favorable twist conformation observed in $\mathbf{5 9}$ and $\mathbf{6 0 .}$ Consequently, the high diastereoselectivity observed could either result from the higher stability of the cis product, or from the requirement of a twist transition state to form the trans product (Fürst-Plattner rule). ${ }^{[32]}$ Furthermore, the higher thermodynamic stability of the C3-cyclization cis adduct $\mathbf{5 7}$ in respect to the N1cyclization cis adduct $\mathbf{5 8}\left(\Delta \mathrm{E}_{57-58}=6.59 \mathrm{Kcal} / \mathrm{mol}\right)$ is in agreement with the observed conversion of $\mathbf{5 8}$ in $\mathbf{5 7}$ via copper catalysis, but not vice-versa. 

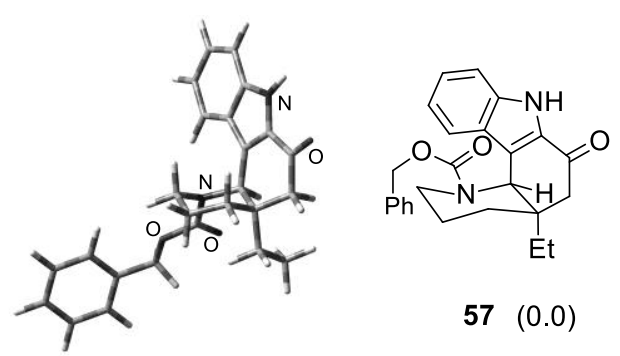

57

$(0.0)$
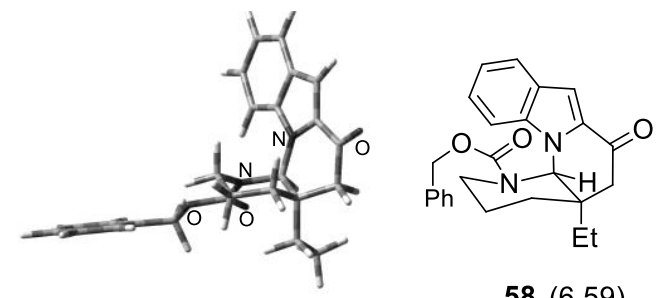

58 (6.59)
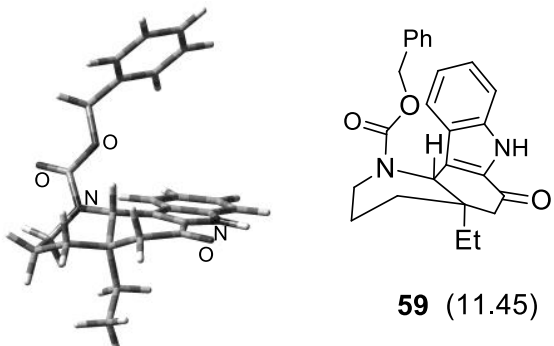

59 (11.45)

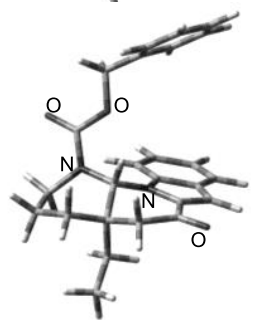

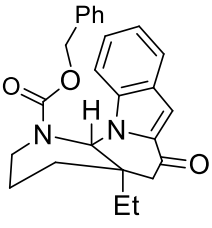

$60(16.56)$

Figure 4. Optimized geometries of the four isomers arising from C3 and N1 cyclization. The energy values [kcal mol $\left.{ }^{-1}\right]$ reported in parentheses are relative to $\mathbf{5 7}$

The C-C cyclization product, after deprotection of the amine, led to a formal total synthesis of aspidospermidine (10), since the free amine had been used in the total synthesis reported by Wenkert. ${ }^{[18 \mathrm{~d}]}$ On the other hand, the scaffold obtained from the N3 attack, corresponded to the core of the gonioma alkaloid goniomitine. Starting from a protected tryptophol derivative, we were able to synthesize goniomitine in 13 linear steps and $11 \%$ overall yield using this cyclization strategy. ${ }^{[7 \mathrm{~g}]}$

Variation of the heterocyclic part was then tested. The synthesized 3-methylindole (17), 2-pyrrole (18), 2-pyran (19) and 2thiophene (20) derivatives were therefore submitted to several cyclization conditions (Scheme 19). All the conditions optimized for the cyclization gave polymerization or $\alpha$-amidoalcohol side products resulting from the attack of water on the iminium intermediate with 3-methylindole (17), 2-pyrrole (18), and 2-thiophene (20) derivatives. On the other hand, dihydropyran derivative 19 gave the cyclic compound after a brief treatment with catalytic $p$ toluenesulfonic acid in acetonitrile. Again, the cyclization gave only the cis diastereoisomer. These results demonstrated that the substitution pattern of the heterocycle is very important for the success of the cyclization reaction with iminium. For future work, it would be important to further modulate the reactivity of the iminium intermediate to extend the scope of the reaction.

When we moved to acyclic carbamate 21, the standard optimized conditions to obtain $\mathrm{C}-\mathrm{C}$ cyclization generated an aldehyde side product derived from the opening of the cyclopropane and hydrolysis of the intermediate iminium (Table 2, entry 1 ). In order to reduce the amount of water, responsible for iminium hydrolysis, we carried out the reaction in presence of molecular sieves (entry 2). After $30 \mathrm{~min}$, the reaction was not complete and we observed the presence of aromatic compound $\mathbf{6 2}$ derived from elimination of benzyl-carbamoylbenzylamide. Fortunately, reducing the acid strength (trifluoroacetic acid) and using a more coordinating solvent such as acetonitrile, we could isolate the C-cyclization product after $90 \mathrm{~min}$ in $63 \%$ isolated yield $(89 \%$ crude yield by NMR, entry 4). Careful control of the reaction time was also important, since the compound $\mathbf{6 3}$ could easily aromatize (entry 5).
By using $p$-toluenesulfonic acid in acetonitrile, it was also possible to obtain aromatic compound $\mathbf{6 2}$ in $36 \%$ yield (entry 6 ). With longer reaction times, the yield was lower due to decomposition (entries 78).
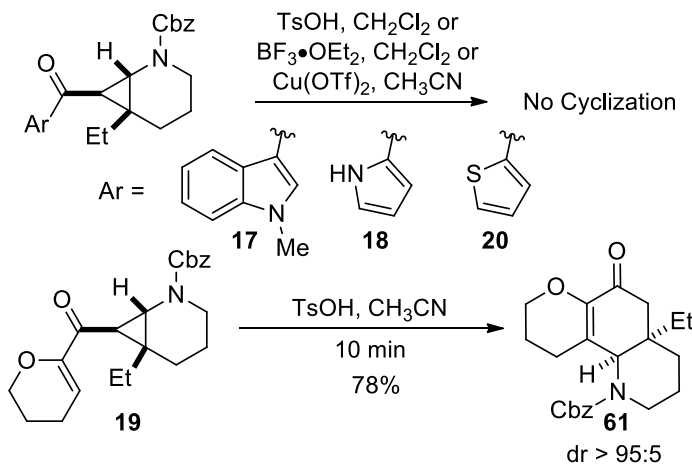

Scheme 19. Influence of the heterocycle structure on the cyclization.

The use of non-protected indole $\mathbf{2 2}$ made control over the reaction even more challenging, as formation of the $\mathrm{C}-\mathrm{N}$ cyclization product was now also possible (Table 3). Nevertheless, we found that the principles discovered in the case of more rigid bicyclic cyclopropanes could also be used in this case. C-Cyclization product $\mathbf{6 5}$ was isolated after only 15 minutes in $70 \%$ yield using $\mathrm{Cu}(\mathrm{OTf})_{2}$ in acetonitrile (entry 2), while the combination of p-toluenesulfonic acid and acetonitrile afforded the C-N cyclization product in $75 \%$ yield (entry 3 ). In the latter case, however, hydrolysis of the formed aminal was observed to give the corresponding half aminal $\mathbf{6 6}$.

Despite the report of Yadav on the cyclization of oxycyclopropane using a large excess of $\mathrm{SnCl}_{4},{ }^{[7 \mathrm{e}]}$ cyclization of the oxycyclopropane derivatives was not efficient under our conditions. Indeed, all the conditions tested with oxycyclopropane $\mathbf{2 3}$ gave side products derived from the water attack on the three-member ring or, in dry conditions, non-defined decomposition, confirming the high sensitivity of this class of compounds observed during the synthesis. 
However, the stoichiometric use of $\mathrm{BF}_{3} \cdot \mathrm{Et}_{2} \mathrm{O}$ in combination with a non coordinating solvent gave a useful yield of $\mathrm{N}$-cyclization product 68 (Scheme 20). The fact that oxycyclopropanes preferred reaction with water or cyclization on nitrogen over C-cyclization is in accordance with a potentially harder oxonium intermediate.

Table 2. Cyclization of acyclic carbamate $\mathbf{2 1}$
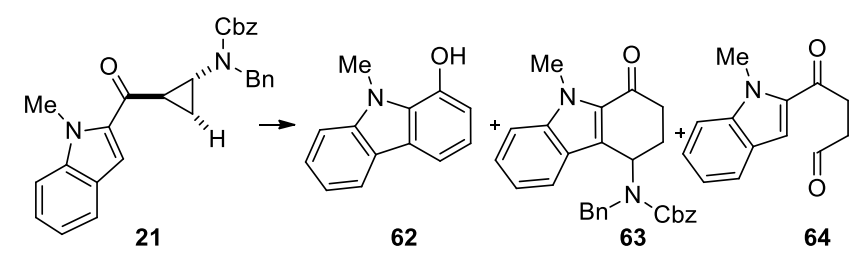

\begin{tabular}{ccccc}
\hline Entry $^{[a]}$ & Catalyst (mol\%) & Solvent & Time & Observed Products \\
\hline 1 & TsOH (20) & $\mathrm{CH}_{2} \mathrm{Cl}_{2}$ & $10 \mathrm{~min}$ & $\mathbf{6 4}$ \\
2 & TsOH (20) & $\mathrm{CH}_{2} \mathrm{Cl}_{2}, \mathrm{MS}$ & $30 \mathrm{~min}$ & $\mathbf{6 2 + 6 3}$ \\
3 & TFA (20) & dry CH $\mathrm{CH}_{3} \mathrm{CN}$ & $30 \mathrm{~min}$ & $\mathbf{2 1}+\mathbf{6 2}$ \\
4 & TFA (20) & dry $\mathrm{CH}_{3} \mathrm{CN}$ & $90 \mathrm{~min}$ & $\mathbf{6 3}(89 \% \text { yield })^{[\mathrm{b}]}$ \\
5 & TFA (20) & dry CH $\mathrm{CH}_{3} \mathrm{CN}$ & $12 \mathrm{~h}$ & $\mathbf{6 3}+\mathbf{6 2}$ \\
6 & TsOH (20) & $\mathrm{CH}_{3} \mathrm{CN}$ & $30 \mathrm{~min}$ & $\mathbf{6 2}(36 \% \text { yield })^{[\mathrm{b}]}$ \\
7 & TsOH (20) & $\mathrm{CH}_{3} \mathrm{CN}$ & $3 \mathrm{~h}$ & $\mathbf{6 2}(20 \% \text { yield })^{[\mathrm{b}]}$ \\
8 & TsOH (20) & $\mathrm{CH}_{2} \mathrm{Cl} l_{2}$ & $3 \mathrm{~h}$ & $\mathbf{6 2}+$ dec. \\
\hline
\end{tabular}

[a] Reaction run with 10-50 mg $21(20-120 \mu \mathrm{mol})$ in $1-5 \mathrm{~mL}$ solvent at rt. [b] Yield calculated by NMR, see supporting information for further details.

Table 3. Selective cyclization of acyclic carbamate $\mathbf{2 2}$

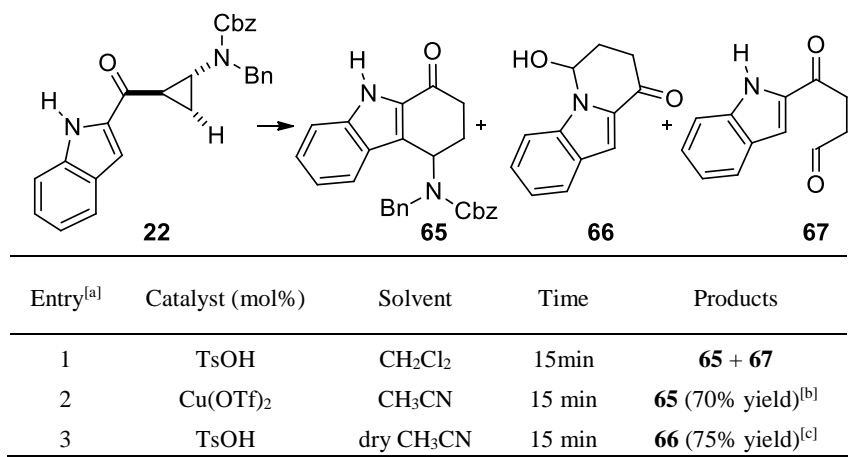

[a] Reaction run with 5-30 mg $22(12-70 \mu \mathrm{mol})$ in 0.5-3 mL solvent, at rt. [b] Calculated by NMR, see supporting information for details. [c] Isolated yield.

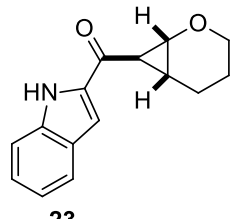

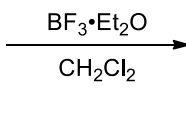

Scheme 20. Cyclization of oxocyclopropane $\mathbf{2 3}$

\section{Conclusion}

In summary, we have shown a further expansion of the scope of cyclization reactions of activated cyclopropanes, including the formal homo-Nazarov process. We proposed two different strategies to modulate the polarization of the three member ring in order to increase its reactivity under catalytic conditions. In the first approach we introduced an ester group on the cyclopropane, allowing an easy access to six-membered rings bearing not only electron-rich, but also electron-poor aromatic substituents. Moreover the presence of an additional carbonyl group allowed a first proof of principle for asymmetric control in the cyclization reaction, probably via a two-points binding interaction with the chiral Lewis acid catalyst.

In a second approach, we studied the influence of heteroatoms on the cyclopropane. The use of aminocyclopropanes in particular was key in the application of the methodology in the total synthesis of natural alkaloids. General synthetic strategies were developed for the synthesis of donor-acceptor cyclopropanes with indole-, pyrrolepyran- or thiophene- ketones as electron-withdrawing groups and cyclic or acyclic carbamates or ethers as donor groups. This broad range of substrates allowed us to better assess the scope and limitation of the methodology, and led to the following conclusions: (1) Both the nucleophilicity and the substitution pattern of the heterocycle were essential for a successful cyclization. If the fit is not ideal, reaction with water or polymerization became favored. (2) For free indole as a nucleophile, the outcome of the cyclization is dependent of the electrophilic character of the formed carbocationic intermediate. Benzylic cation gave C3 cyclization exclusively, whereas oxonium favored N1 cyclization. For iminium intermediates, it was possible to switch the regioselectivity using a copper catalyst in acetonitrile for $\mathrm{C} 3$ cyclization and ptoluenesulfonic acid in dichloromethane for N1 cyclization. Both experiments and calculations showed that $\mathrm{C} 3$ cyclization led to the thermodynamic product. (3) Acyclic carbamates could be successfully cyclized, but they are more sensitive substrates. Mild conditions are required to prevent a facile aromatization of the product. This last reaction manifold could have potential for the synthesis of substituted carbazoles, however.

In conclusion we have shown the versatility of the formal homoNazarov reaction and other cyclization processes using keto-ester activated cyclopropanes, aminocyclopropanes and oxycyclopropanes. We extended the scope of the reaction and we showed a first preliminary example of asymmetric induction. Currently, our research in this transformation is focusing in new applications in total synthesis, as well as increasing the enantioselectivity by the use of other catalysts.

\section{Acknowledgements}

The EPFL is acknowledged for financial support. Dr. Fides Benfatti is a recipient of IEF Marie Curie fellowship (Nr. 253274). The following EPFL students and technical assistants are thanked for the synthesis of starting material, chiral ligands or reagents: Ms. Letitzia Gullifa, Mr Frederic Steimer and Mr. Valentin Manzanares.

[1] a) Cycloadditions Reactions in Organic Synthesis, S. Kobayashi, K. A. Jorgensen, eds., Wiley VCH, Weinheim, Germany, 2002. b) S. M. Ma, Handbook of Cyclization Reactions, WILEY-VCH, Weinheim, 2009.

[2] I. N. Nazarov, I. I. Zaretskaya, Izv. Akad. Nauk. SSSR. Ser. Khim 1941, 211.

[3] K. L. Habermas, S. E. Denmark, T. K. Jones, Org. React. (N. Y.) 1994, 45, 1

[4] a) S. Giese, F. G. West, Tetrahedron 2000, 56, 10221. b) Y. Wang, B. D. Schill, A. M. Arif, F. G. West, Org. Lett. 2003, 5, 2747. c) C. Bee, E. Leclerc, M. A. Tius, Org. Lett. 2003, 5, 4927. d) W. He, X. F. Sun, A. J. Frontier, J. Am. Chem. Soc. 2003, 125, 14278. e) M. Janka, W. He, A. J. Frontier, R. Eisenberg, J. Am. Chem. Soc. 2004, 126, 6864. f) W. A. Batson, D. Sethumadhavan, M. A. Tius, Org. Lett. 2005, 7, 2771. g) J. A. Malona, J. M. Colbourne, A. J. Frontier, Org. Lett. 2006, 8, 5661. h) L. Zhang, S. Wang, J. Am. Chem. Soc. 2006, 128, 1442. i) J. M. Tang, S. Bhunia, S. M. A. Sohel, M. Y. Lin, H. Y. Liao, S. Datta, A. Das, 
R. S. Liu, J. Am. Chem. Soc. 2007, 129, 15677. j) I. Walz, A. Bertogg, A. Togni, Eur. J. Org. Chem. 2007, 2650. k) W. He, I. R. Herrick, T. A. Atesin, P. A Caruana, C. A. Kellenberger, A. J. Frontier, J. Am. Chem. Soc. 2008, 130, 1003. 1) M. Kawatsura, Y. Higuchi, S. Hayase, M. Nanjo, T. Itoh, Synlett, 2008, 8 , 1009. m) A. P. Marcus, A. S. Lee, R. L. Davis, D. J. Tantillo, R. Sarpong, Angew. Chem. Int. Ed. 2008, 47, 6379. n) M. Amere, J. Blanchet, M. C. Lasne, J. Rouden, Tetrahedron Lett. 2008, 49, 2541. o) T. Jin, Y. Yamamoto, Org. Lett. 2008, 10, 3137. p) G. Lemiere, V. Gandon, K. Cariou, A. Hours, T. Fukuyama, A. L. Dhimane, L. Fensterbank, M. Malacria, J. Am. Chem. Soc. 2009, 131 2993. q) T. Vaidya, A. C. Atesin, I. R. Herrick, A. J. Frontier, R. Eisenberg, Angew. Chem., Int. Ed. 2010, 49, 3363. r) P. A. Wender, R. T. Stemmler, L. E. Sirois, J. Am. Chem. Soc. 2010, 132, 2532. For reviews, see: s) M. A. Tius, Eur J. Org. Chem. 2005, 2193. t) A. J. Frontier, C. Collison, Tetrahedron 2005, 61, 7577. u) H. Pellissier, Tetrahedron 2005, 61, 6479. v) W. Nakanishi, F. G. West Curr. Opin. Drug Discov. Dev. 2009, 12, 732. w) S. Thompson, A. G. Coyne, P. C. Knipe, M. D. Smith, Chem. Soc. Rev. 2011, 40, 4217. y) T. Vaidya, R. Eisenberg, A. J. Frontier, ChemCatChem, 2011, DOI: 10.1002/cctc.201100137.

[5] a) V. K. Aggarwal, A. J. Beffield, Org. Lett. 2003, 5, 5075. b) G. X. Liang, S. N Gradl, D. Trauner, Org. Lett. 2003, 5, 4931. c) G. X. Liang, D. Trauner, J. Am. Chem. Soc. 2004, 126, 9544. d) I. Walz, A. Togni, Chem. Commun. 2008, 4315. e) M. Rueping, W. Ieawsuwan, A. P. Antonchick, B. J. Nachtsheim, Angew. Chem., Int. Ed. 2007, 46, 2097. f) Cao, P.; Deng, C.; Zhou, Y. Y.; Sun, X. L.; Zheng, J. C.; Xie, Z. W.; Tang, Y., Angew. Chem., Int. Ed. 2010, 49, 4463. g) Basak, A. K.; Shimada, N.; Bow, W. F.; Vicic, D. A.; Tius, M. A., J. Am. Chem Soc. 2010, 132, 8266

[6] A. D. Walsh, Nature 1947, 159, 712.

[7] a) W. S. Murphy, S. Wattanasin, Tetrahedron Lett. 1980, 21, 1887. b) W. S Murphy, S. Wattanasin, J. Chem. Soc. Perkin Trans. 1 1981, 2920. c) W. S Murphy, S. Wattanasin, J. Chem. Soc. Perkin Trans. 1 1982, 1029. d) O. Tsuge, S. Kanemasa, T. Otsuka, T. Suzuki, Bull. Chem. Soc. Jpn. 1988, 61, 2897. e) V. K. Yadav, N. V. Kumar, Chem. Commun. 2008, 3774. f) F. De Simone, J. Andres, R. Torosantucci, J. Waser, Org. Lett. 2009, 11, 1023. g) F. De Simone, J. Gertsch, J. Waser, Angew. Chem., Int. Ed. 2010, 49, 5767. h) D. V. Patil, L. H. Phun, S. France, Org. Lett. 2010, 12, 5684. i) L. H. Phun, D. V. Patil, M. A. Cavitt, S. France, Org. Lett. 2011, 13, 1952. For a review, see: j) F. De Simone, J. Waser, Chimia 2009, 63, 162.

[8] a) Patil, D. V.; Cavitt, M. A.; Grzybowski, P.; France, S., Chem. Commun. 2011 47, 10278. b) Patil, D. V.; Cavitt, M. A.; France, S., Heterocycles. 2011, DOI 10.3987/COM-11-S(P)87.

[9] a) H. U. Reissig, R. Zimmer, Chem. Rev. 2003, 103, 1151. b) F. Gnad, O Reiser, Chem. Rev. 2003, 103, 1603. c) M. Yu, B. L. Pagenkopf, Tetrahedron 2005, 61, 321. d) C. A. Carson, M. A. Kerr, Chem. Soc. Rev. 2009, 38, 3051. e) F. De Simone, J. Waser, Synthesis 2009, 3353. f) T. P. Lebold, M. A. Kerr, Pure Appl. Chem. 2010, 82, 1797. g) T. F. Schneider, D. B. Werz, Org. Lett. 2011, $13,1848$.

[10] a) G. Stork, M. Marx, J. Am. Chem. Soc. 1969, 91, 2371. b) G. Stork, M Gregson, M. J. Am. Chem. Soc. 1969, 91, 2373. c) G. Stork, P. A. Grieco, J. Am Chem. Soc. 1969, 91, 2407. d) E. J. Corey, R. D. Balanson, Tetrahedron Lett. 1973, 3153. e) S. Danishefsky, Acc. Chem. Res. 1979, 12, 66.

[11] This work was part of the master thesis of Mr. Tanguy Saget (April 2009September 2009) in our laboratory.

[12] a) P. D. Pohlhaus, S. D. Sanders, A. T. Parsons, W. Li, J. S. Johnson, J. Am Chem. Soc. 2008, 130, 8642. b) A. T. Parsons, J. S. Johnson, J. Am. Chem. Soc. 2009, 131, 14202.

[13] P. Wyatt, A. Hudson, J. Charmant, A. G. Orpen, H. Phetmung, Org. Biomol Chem. 2006, 4, 2218.

[14] F. Gonzalez-Bobes, M. D. B. Fenster, S. Kiau, L. Kolla, S. Kolotuchin, M Soumeillant, Adv. Synth. Catal. 2008, 350, 813.
[15] C. Perreault, S. R. Goudreau, L. E. Zimmer, A. B. Charette, Org. Lett. 2008, 10, 689

[16] M. D. Ganton, M. A. Kerr J. Org. Chem. 2004, 69, 8554.

[17] See supporting information for a detailed procedure.

[18] a) E. Wenkert, T. Hudlicky, H. D. H. Showalter, J. Am. Chem. Soc. 1978, 100, 4893. b) E. Wenkert, Acc. Chem. Res. 1980, 13, 27. c) E. Wenkert, T. D. J. Halls, L. D. Kwart, G. Magnusson, H. D. H. Showalter, Tetrahedron 1981, 37 , 4017. d) E. Wenkert, T. Hudlicky, J. Org. Chem. 1988, 53, 1953.

[19] L. Larquetoux, N. Ouhamou, A. Chiaroni, Y. Six, Eur. J. Org. Chem. 2005, 4654.

[20] P. A. Grieco, M. D. Kaufman, J. Org. Chem. 1999, 64, 7586.

[21] The choice of coupling agent was important when considering the instability of the aminocyclopropanes. The coupling involving DMTMM has the added advantage of simple purification during work up without requiring chromatographic purification

[22] A. R. Katritsky, K. Akutagawa, Tetrahedron Lett. 1985, 26, 5935.

[23] a) G. M. Wieber, L. S. Hegedus, B. Akermark, E. T. Michalson, J. Org. Chem. 1989, 54, 4649. b) A. Kamatani, L. E. Overman, J. Org. Chem. 1999, 64, 8743.

[24] See supporting information for further details

[25] In contrast to the case of cyclic enamide, this cyclopropanation was best done at higher temperature and with a fast addition of carbene precursor. Furthermore, isomerization of the oxycyclopropane was faster.

[26] Yield of $\mathbf{2 3}$ is based on the amount of pure $\mathbf{5 4}$ in the starting material.

[27] See Ref. $7 \mathrm{~g}$ for a more detailed discussion of the optimization of the cyclization reaction.

[28] B. Schlegel, J. Comput. Chem. 2003, 24, 1514.

[29] C. Peng, P. Y. Ayala, H. B. Schlegel, M. J. Frisch, J. Comput. Chem. 1996, 17, 49.

[30] M. J. Frisch, G. W. Trucks, H. B. Schlegel, G. E. Scuseria, M. A. Robb, J. R. Cheeseman, G. Scalmani, V. Barone, B. Mennucci, G. A. Petersson, H. Nakatsuji, M. Caricato, X. Li, H. P. Hratchian, A. F. Izmaylov, J. Bloino, G. Zheng, J. L. Sonnenberg, M. Hada, M. Ehara, K. Toyota, R. Fukuda, J. Hasegawa, M. Ishida, T. Nakajima, Y. Honda, O. Kitao, H. Nakai, T. Vreven, J. A. Montgomery, Jr., J. E. Peralta, F. Ogliaro, M. Bearpark, J. J. Heyd, E. Brothers, K. N. Kudin, V. N. Staroverov, R. Kobayashi, J. Normand, K. Raghavachari, A. Rendell, J. C. Burant, S. S. Iyengar, J. Tomasi, M. Cossi, N. Rega, J. M. Millam, M. Klene, J. E. Knox, J. B. Cross, V. Bakken, C. Adamo, J. Jaramillo, R. Gomperts, R. E. Stratmann, O. Yazyev, A. J. Austin, R. Cammi, C. Pomelli, J. W. Ochterski, R. L. Martin, K. Morokuma, V. G. Zakrzewski, G. A. Voth, P. Salvador, J. J. Dannenberg, S. Dapprich, A. D. Daniels, Ö. Farkas, J. B. Foresman, J. V. Ortiz, J. Cioslowski, and D. J. Fox, Gaussian, Inc., Wallingford CT, 2009.

[31] J. Tomasi, B. Mennucci, R. Cammi, Chem. Rev. 2005, 105, 2999.

[32] F. De Simone, J. Waser, Synlett 2011, 5, 589.

Received: ((will be filled in by the editorial staff)) Revised: ((will be filled in by the editorial staff)) Published online: ((will be filled in by the editorial staff)) 
Cyclization Reactions

Filippo De Simone, Tanguy Saget, Fides Benfatti, Sofia Almeida and

Jérôme Waser* Page -

Page

Formal homo-Nazarov and other Cyclizations Reactions of Activated Cyclopropanes

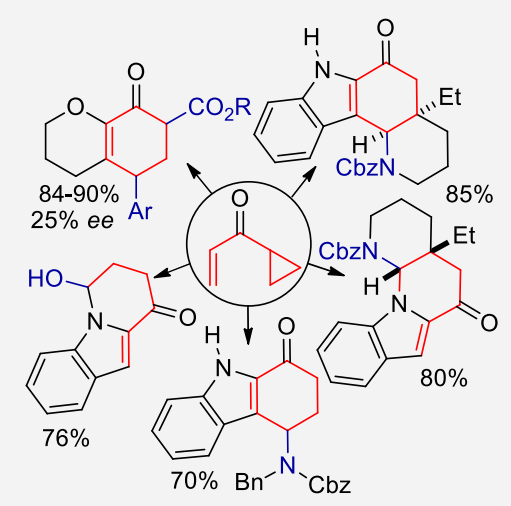

Push and Pull: The extension of the scope of cyclization reactions of donor-acceptor cyclopropanes with particular focus on the catalytic formal homo-Nazarov process is reported. An ester group or a heteroatom was introduced on the cyclopropane to enhance its acceptor or donor-mediated polarization. Control over regio- and diastereoselectivity could be achieved, and a first proof of principle for asymmetric induction is reported. 


\section{Supporting information}

\section{Formal homo-Nazarov and other Cyclizations Reactions of Activated Cyclopropanes}

Filippo De Simone, Tanguy Saget, Fides Benfatti, Sofia Almeida and Jérôme Waser*

124 pages

1. General Methods

2. General procedures S3

3. Ketoesters synthesis S5

4. Cyclization of ketoesters $\quad$ S16

5. Asymmetric induction $\quad$ S22

6. Amino- and oxycyclopropanes synthesis S26

7. Cyclization of amino- and oxycyclopropanes S53

8. Computational data $\quad$ S64

9. Spectra of new compounds $\quad$ S69 


\section{General Methods}

All reactions were carried out in oven dried glassware under an atmosphere of nitrogen, unless stated otherwise. For quantitative flash chromatography technical grade solvents were used. For flash chromatography for analysis, HPLC grade solvents from Sigma-Aldrich were used. THF, $\mathrm{Et}_{2} \mathrm{O}, \mathrm{CH}_{3} \mathrm{CN}$, toluene, hexane and $\mathrm{CH}_{2} \mathrm{Cl}_{2}$ were dried by passage over activated alumina under nitrogen atmosphere $\left(\mathrm{H}_{2} \mathrm{O}\right.$ content $<10 \mathrm{ppm}$, Karl-Fischer titration). $\mathrm{NEt}_{3}$ and pyridine were distilled under nitrogen from $\mathrm{KOH}$. All chemicals were purchased from Acros, Aldrich; Fluka, VWR, Aplichem or Merck and used as such unless stated otherwise. Chromatographic purification was performed as flash chromatography using Macherey-Nagel silica 40-63, $60 \AA$, using the solvents indicated as eluent with 0.1-0.5 bar pressure. The deactivation of silica was obtained with a $1 \%$ solution of $\mathrm{Et}_{3} \mathrm{~N}$ in the indicated solvent. TLC was performed on Merck silica gel $60 \mathrm{~F}_{254}$ TLC glass plates or aluminium plates and visualized with UV light, permanganate stain, CAN stain or Anisaldehyde stain. Melting points were measured on a calibratedBüchi B-540 melting point apparatus using open glass capillaries. ${ }^{1} \mathrm{H}-\mathrm{NMR}$ spectra were recorded on a Brucker DPX-400 $400 \mathrm{MHz}$ and a Brucker AV-500 500 MHz spectrometer in chloroform-d, DMSO- $\mathrm{d}_{6}$ or $\mathrm{CD}_{3} \mathrm{OD}$, all signals are reported in ppm with the internal chloroform signal at $7.26 \mathrm{ppm}$, the internal DMSO signal at $2.50 \mathrm{ppm}$ or the internal methanol signal at $3.30 \mathrm{ppm}$ as standard. The data is being reported as $(\mathrm{s}=$ singlet, $\mathrm{d}=$ doublet, $\mathrm{t}=$ triplet, $\mathrm{q}=$ quadruplet, $\mathrm{qi}=$ quintet, $\mathrm{m}=$ multiplet or unresolved, $\mathrm{br}=$ broad signal, $\mathrm{app}=$ apparent, coupling constant(s) in $\mathrm{Hz}$, integration; interpretation). ${ }^{13} \mathrm{C}-\mathrm{NMR}$ spectra were recorded with ${ }^{1} \mathrm{H}$-decoupling on a Brucker DPX-400 $100 \mathrm{MHz}$ and a Brucker AV-500 $125 \mathrm{MHz}$ spectrometer in chloroform- $\mathrm{d}, \mathrm{DMSO}-\mathrm{d}_{6}$ or $\mathrm{CD}_{3} \mathrm{OD}$, all signals are reported in ppm with the internal chloroform signal at $77.0 \mathrm{ppm}$, the internal DMSO signal at $39.5 \mathrm{ppm}$ or the internal methanol signal at $49.0 \mathrm{ppm}$ as standard. Infrared spectra were recorded on a JASCO FT-IR B4100 spectrophotometer with an ATR PRO410-S and a ZnSe prisma and are reported as $\mathrm{cm}^{-1}$ ( $\mathrm{w}=$ weak, $\mathrm{m}=$ medium, $\mathrm{s}=$ strong, $\mathrm{br}=$ broad). Gas chromatographic and low resolution mass spectrometric measurements were performed on a Perkin-Elmer Clarus 600 gas chromatographer and mass spectrometer using a Perkin-Elmer Elite fused silica column (length: $30 \mathrm{~m}$, diameter: $0.32 \mathrm{~mm}$ ) and Helium as carrier gas. High resolution mass spectrometric measurements were performed by the mass spectrometry service of ISIC at the EPFL on a MICROMASS (ESI) Q-TOF Ultima API. HPLC measurement were done on a JASCO HPLC system with an AS2055 Autosampler, a PU 2089 Pump, a UV 2075 detector and a SEDEX 85 
(SEDERE) detector using a CHIRALPAK IC column from DAICEL Chemical Industries Ltd. HPLC grade solvents from Sigma-Aldrich were used.

\section{General Procedures}

\section{General procedure 1 (GP1): cyclopropanation}

Following a reported procedure, ${ }^{[1]} \mathrm{Rh}_{2}(\mathrm{esp})_{2}(0.1 \mathrm{~mol} \%)$ was loaded in a flask in the glovebox. A solution of alkene (1 equiv, 1.2 $\mathrm{M}$ in DCM) (freshly filtered over a pad of aluminum oxide) was then added and the reaction mixture was stirred at $0^{\circ} \mathrm{C}$. After $5 \mathrm{~min}$, a solution of diazomalonate (1 equiv, $1.2 \mathrm{M}$ in DCM) was added. The resulting mixture was stirred at $0^{\circ} \mathrm{C}$ for $10 \mathrm{~min}$ and then stirred overnight at $23^{\circ} \mathrm{C}$. The reaction mixture was then concentrated under reduced pressure and purified directly by column chromatography.

\section{General procedure 2 (GP2): selective saponification}

Following a reported procedure, ${ }^{[2]} 1,1$-cyclopropane diester (1 equiv) was dissolved in $\mathrm{MeOH}$, $1.7 \mathrm{~N}$ aqueous $\mathrm{NaOH}$ (1.2 equiv) and some drops of THF. The reaction mixture was stirred for $1.5 \mathrm{~h}$ and was then diluted with DCM and water. The layers were separated. The $\mathrm{pH} 14$ aqueous solution was washed one more time with DCM, acidified with $1 \mathrm{~N} \mathrm{HCl}$ to reach $\mathrm{pH}<1$ and then extracted with DCM (x4). The organic layers were combined, dried over $\mathrm{MgSO}_{4}$ and evaporated under reduced pressure to afford the corresponding acid as a white solid which was pure enough to be engaged in the next step without further purification.

\section{General procedure 3 (GP3): coupling}

The previously formed acid ( 1 equiv) was dissolved in THF and cooled to $-95^{\circ} \mathrm{C}$. Dihydropyran (2.2 equiv) was dissolved in THF and the mixture was cooled to $-78^{\circ} \mathrm{C}$. ${ }^{t} \mathrm{BuLi}(1.6 \mathrm{M}$ solution in pentane, 2.2 equiv) was added dropwise to this solution at the same temperature. The reaction mixture was stirred at $0^{\circ} \mathrm{C}$ for $30 \mathrm{~min}$, diluted with THF and cooled to $-95^{\circ} \mathrm{C}$. This solution was then added via cannula to the cooled solution of the acid and the resulting mixture was stirred until the cooling bath reached $-50{ }^{\circ} \mathrm{C}$. The reaction mixture was then poured in water and diluted with DCM. The layers were separated and the organic layer was extracted with dilute aqueous solution of $\mathrm{NaOH}(\mathrm{pH}>13)(\mathrm{x} 3)$. The aqueous layers were combined and acidified with $1 \mathrm{~N}$

[1] Gonzalez-Bobes, F.; Fenster, M. D. B.; Kiau, S.; Kolla, L.; Kolotuchin, S.; Soumeillant, M. Adv. Synth. Catal. 2008, 350, 813 .

[2] Perreault, C.; Goudreau, S. R.; Zimmer, L. E.; Charette, A. B. Org. Lett. 2008, 10, 689. 
$\mathrm{HCl}$ to reach $\mathrm{pH}<1$. The aqueous layer was extracted with DCM (x5). The organic layers were combined, dried over $\mathrm{MgSO}_{4}$ and evaporated under reduced pressure to give an oil which was dissolved in DMF. To this solution were added $\mathrm{K}_{2} \mathrm{CO}_{3}$ (2 equiv) and MeI (5 equiv) and the reaction mixture was stirred for 2 days at $23^{\circ} \mathrm{C}$. Then, AcOEt was added. The organic layer was washed with brine (x5), dried over $\mathrm{MgSO}_{4}$ and evaporated. The crude product was purified by column chromatography (PET/Et $2 \mathrm{O} 8: 1$ to $3: 1$ ) to afford the desired homo-Nazarov substrate as a single diastereoisomer.

\section{General procedure 4 (GP4): homo-Nazarov cyclization}

Toluenesulfonic acid ( 0.2 equiv) was added to a solution of vinyl cyclopropyl ketone ( 1 equiv, $0.04 \mathrm{M}$ in anhydrous $\mathrm{CH}_{3} \mathrm{CN}$ ) at room temperature. The reaction was stirred during the indicated time. The solution was quenched with $\mathrm{NaHCO}_{3}$ and extracted with $\mathrm{Et}_{2} \mathrm{O}$. The combined organic layers were washed with brine, dried over $\mathrm{MgSO}_{4}$ and the solvent was removed under reduced pressure. The crude product was purified as indicated.

\section{General procedure 5 (GP5): hydrogenolysis}

$\mathrm{Pd} / \mathrm{C}$ (0.10 equiv) was added portionswise to a solution $(0.02 \mathrm{M}$ in $\mathrm{EtOH})$ of protected amine at room temperature. Hydrogen gas was bubbled into the solution until the conversion of all starting material (controlled by TLC) ${ }^{[3]}$. The suspension was filtered on celite (pre-washed with DCM), washed with DCM and AcOEt and dried over $\mathrm{MgSO}_{4}$. The organic layer was evaporated on reduced pressure. No further purification was needed.

\section{General procedure 6 (GP6): Carboxylation of indole or pyrrole}

Using a slight modification of a reported procedure, ${ }^{[4]}{ }^{n} \mathrm{BuLi}$ (2.5 $\mathrm{M}$ in pentane, 1.2 equiv) was added dropwise to a solution of indole or pyrrole $\left(0.2 \mathrm{M}\right.$ in $\left.\mathrm{Et}_{2} \mathrm{O}\right)$ at $0^{\circ} \mathrm{C}$. The reaction was refluxed 2 hours then cooled to $0^{\circ} \mathrm{C}$ and $\mathrm{CO}_{2}$ was bubbled in the solution during 30 minutes. The suspension was quenched with water and the organic layer was washed several times with water. The aqueous layer was acidified until $\mathrm{pH}=2$ and the precipitate was filtered and dried under vacuum.

[3] A batch to batch dependency of the reaction time was observed (15 min to $5 \mathrm{~h}$ ). It is consequently important to monitor the reaction carefully via TLC.

[4] D. A Shirley, P. A. Roussel, J. Am. Chem. Soc. 1953, 75, 375. 


\section{Ketoesters synthesis}

\section{(E)- $N$-Methoxy- $N$-methyl-3-(4-methoxyphenyl)-acrylamide (69) and $N$-methoxy- $N$-methyl-}

\section{1-[2-(4-methoxyphenyl)-cyclopropan-1-yl]-formamide (71)}

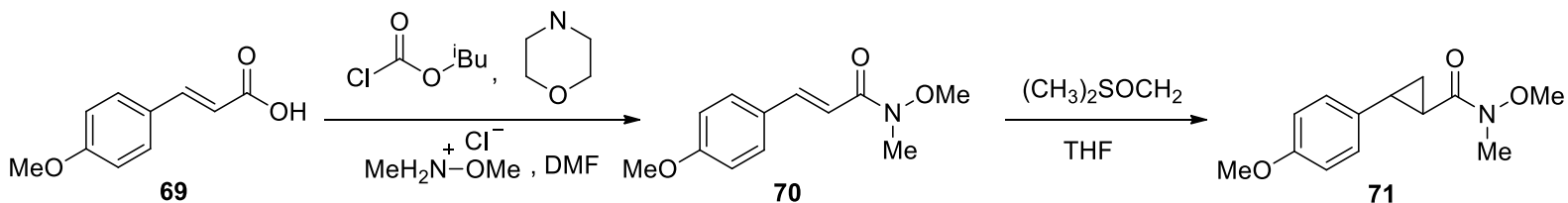

Following the reported procedure ${ }^{[5]} N$-methylmorpholine $(1.36 \mathrm{~mL}, 12.3 \mathrm{mmol}, 1.10$ equiv) was added to a solution of acid $68\left(2.00 \mathrm{~g}, 11.2 \mathrm{mmol}, 1.00\right.$ equiv) in DMF (15 mL) at $0{ }^{\circ} \mathrm{C}$. After 25 min, iso-butylchloroformate $\left(1.61 \mathrm{~mL}, 12.3 \mathrm{mmol}, 1.10\right.$ equiv) was added dropwise at $0{ }^{\circ} \mathrm{C}$. After $10 \mathrm{~min}, \mathrm{~N}, O$-dimethylhydroxylamine hydrochloride $(1.20 \mathrm{~g}, 12.3 \mathrm{mmol}, 1.10$ equiv) was added, followed by $N$-methylmorpholine $(1.61 \mathrm{~mL}, 14.6 \mathrm{mmol}, 1.30$ equiv) and the reaction mixture was warmed to $23^{\circ} \mathrm{C}$. After $6 \mathrm{~h}$, the reaction was quenched with $0.5 \mathrm{M} \mathrm{HCl}(20 \mathrm{~mL})$ and extracted with $\mathrm{CH}_{2} \mathrm{Cl}_{2}(3 \times 20 \mathrm{~mL})$. The combined organic layers were washed with $0.5 \mathrm{M} \mathrm{NaOH}$ $(2 \times 20 \mathrm{~mL})$, brine $(1 \times 20 \mathrm{~mL})$, dried over $\mathrm{MgSO}_{4}$ and the solvent was removed under reduced pressure. After $30 \mathrm{~min}$ in high vacuum, the residues were dissolved in $\mathrm{Et}_{2} \mathrm{O}(60 \mathrm{~mL})$ and washed with brine $(2 \times 30 \mathrm{~mL})$, dried over $\mathrm{MgSO}_{4}$ and the solvent was removed under reduced pressure to give the Weinreb amide $\mathbf{7 0}$ which was used directly without purification.

A solution of ylide ${ }^{[6]}(12.2 \mathrm{~mL}, 6.58 \mathrm{mmol}, 1.20 \mathrm{eq})$ in anhydrous THF was added dropwise to a solution of amide 70 (1.21 g, $5.47 \mathrm{mmol}, 1.00$ equiv) in THF (45 mL) at RT under nitrogen. The reaction was stirred at $40^{\circ} \mathrm{C}$ during $2 \mathrm{~h}$ then quenched with $\mathrm{NaHCO}_{3}(50 \mathrm{~mL})$ and extracted with $\mathrm{Et}_{2} \mathrm{O}(3 \times 50 \mathrm{~mL})$. The combined organic layers were washed with brine $(2 \times 30 \mathrm{~mL})$, dried over $\mathrm{MgSO}_{4}$ and the solvent was removed under reduced pressure. Purification by flash column chromatography (PET/AcOEt, 7:3) afforded 71 (824 mg, 3.50 mmol, $64 \%$ ) over 2 steps as oil.

$R_{f} 0.35$ (PET/AcOEt 7:3, Anisaldehyde).

[5] Nagarajan, S. R.; Lu, H. F.; Gasiecki, A. F.; Khanna, I. K.; Parikh, M. D.; Desai, B. N.; Rogers, T. E.; Clare, M.; Chen, B. B.; Russell, M. A.; Keene, J. L.; Duffin, T.; Engleman, V. W.; Finn, M. B.; Freeman, S. K.; Klover, J. A.; Nickols, G. A.; Nickols, M. A.; Shannon, K. E.; Steininger, C. A.; Westlin, W. F.; Westlin, M. M.; Williams, M. L. Bioorg. Med. Chem. 2007, 15, 3390.

[6] $n \mathrm{BuLi}$ (2.5 M, 1.0 equiv) was added dropwise to a solution of trimethylsufoxonium iodide (1.1 equiv) in anhydrous THF $(0.75 \mathrm{M})$ at $0^{\circ} \mathrm{C}$. The solution was allowed to warm to RT and stirring was continued under nitrogen for 1 hour. A solution $0.54 \mathrm{M}$ of ylide was obtained. 
${ }^{1} \mathrm{H}$ NMR $\left(\mathrm{CDCl}_{3}, 400 \mathrm{MHz}\right) \delta 7.06(\mathrm{~d}, J=8.6 \mathrm{~Hz}, 2 \mathrm{H} ; \mathrm{ArH}), 6.83(\mathrm{~d}, J=8.7 \mathrm{~Hz}, 2 \mathrm{H} ; \mathrm{ArH})$, 3.78 (s, 3H; $\left.\mathrm{OCH}_{3}\right), 3.69\left(\mathrm{~s}, 3 \mathrm{H} ; \mathrm{OCH}_{3}\right), 3.23\left(\mathrm{~s}, 3 \mathrm{H} ; \mathrm{NCH}_{3}\right), 2.50-2.42(\mathrm{~m}, 1 \mathrm{H} ; \mathrm{CH}), 2.33(\mathrm{~m}$, $1 \mathrm{H} ; \mathrm{CH}), 1.65-1.55$ (m, $\left.1 \mathrm{H} ; \mathrm{CH}_{2}\right), 1.30-1.22$ (m, $\left.1 \mathrm{H} ; \mathrm{CH}_{2}\right)$.

${ }^{1} \mathrm{H}$ NMR spectra corresponded to the literature values. ${ }^{[7]}$

\section{(E)-2-[2-(4-Methoxyphenyl)-1-cyclopropanecarbonyl]-5,6-dihydro-4H-pyran (6a)}

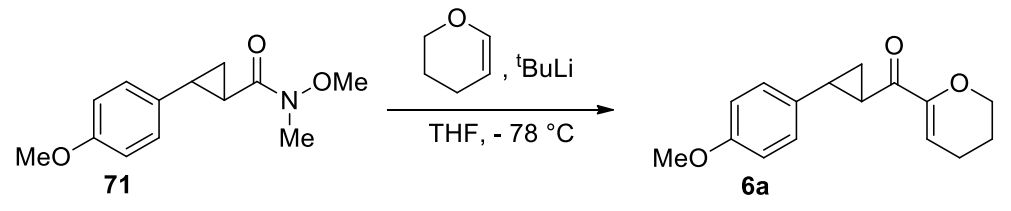

Following a slight modification of a reported procedure, ${ }^{[7]} t \mathrm{BuLi}(0.85 \mathrm{~mL}, 1.4 \mathrm{mmol}, 2.0$ equiv) was added dropwise in a solution of dihydropyran 44 ( $0.14 \mathrm{~mL}, 1.5 \mathrm{mmol}, 2.2$ equiv) in THF (15 $\mathrm{mL})$ at $-78^{\circ} \mathrm{C}$. The flask was transferred in a bath of ice. After $30 \mathrm{~min}$ at $0^{\circ} \mathrm{C}$ the reaction was cooled to $-78^{\circ} \mathrm{C}$ and a solution of Weinreb amide 71 ( $0.16 \mathrm{~g}, 0.68 \mathrm{mmol}, 1.0$ equiv) in THF (14 $\mathrm{mL}$ ) was added slowly dropwise. The reaction was stirred at $-78^{\circ} \mathrm{C}$ during $2 \mathrm{~h}$ and 15 min then warmed at $0^{\circ} \mathrm{C}$ and quenched with saturate solution of $\mathrm{NH}_{4} \mathrm{Cl}(50 \mathrm{~mL})$. The product was extracted with $\mathrm{Et}_{2} \mathrm{O}(3 \times 50 \mathrm{~mL})$ and washed with brine $(2 \times 50 \mathrm{~mL})$, dried over $\mathrm{MgSO}_{4}$ and concentrated under reduced pressure to give $\mathbf{6 a}(105 \mathrm{mg}, 410 \mu \mathrm{mol}, 60 \%)$ after purification via flash chromatography (PET/AcOEt, 7:3) as yellow oil.

$R_{f} 0.70$ (PET/AcOEt 7:3, Anisaldehyde).

${ }^{1} \mathrm{H} \mathrm{NMR}\left(\mathrm{CDCl}_{3}, 400 \mathrm{MHz}\right) \delta 7.06(\mathrm{~d}, J=8.6 \mathrm{~Hz}, 2 \mathrm{H} ; \mathrm{ArH}), 6.83(\mathrm{~d}, J=8.7 \mathrm{~Hz}, 2 \mathrm{H} ; \mathrm{ArH})$, $6.01\left(\mathrm{t}, J=4.2 \mathrm{~Hz}, 1 \mathrm{H}\right.$; alkeneH), $4.14-4.06\left(\mathrm{~m}, 2 \mathrm{H} ; \mathrm{CH}_{2} \mathrm{O}\right), 3.79$ (s, 3H; $\mathrm{OCH}_{3}$ ), 2.71-2.59 (m, 1H; cyclopropaneH), 2.54 (ddd, $J=4.1,6.6,10.5 \mathrm{~Hz}, 1 \mathrm{H}$; cyclopropaneH), 2.22 (dt, $J=4.4$, $\left.6.3 \mathrm{~Hz}, 2 \mathrm{H} ; \mathrm{CH}_{2} \mathrm{CH}_{2}\right), 1.92-1.81$ (m, 2H; $\mathrm{CH}_{2} \mathrm{CH}_{2}$ ), $1.76-1.67$ (m, 1H; cyclopropaneH), 1.37 (ddd, $J=4.0,6.8,8.0 \mathrm{~Hz}, 1 \mathrm{H}$; cyclopropaneH).

${ }^{13} \mathrm{C} \mathrm{NMR}\left(\mathrm{CDCl}_{3}, 100 \mathrm{MHz}\right) \delta 194.6,158.2,151.4,132.5,127.2,113.8,109.5,66.3,55.2,29.3$, 27.30, 21.4, 20.7, 19.2 .

[7] Boeckman, R. K.; Bruza, K. J. Tetrahedron 1981, 37, 3997. 
IR $v\left(\mathrm{~cm}^{-1}\right)$ : $3036(\mathrm{w}), 2950(\mathrm{w}), 2934(\mathrm{w}), 2836(\mathrm{w}), 1681(\mathrm{~m}), 1667(\mathrm{~m}), 1625(\mathrm{~s}), 1516(\mathrm{~s})$, 1440 (m), 1393 (m), 1331 (m), 1286 (s), 1248 (s), 1237 (m), 1201 (w), 1180 (s), 1091 (m), 1061 (s), $1032(\mathrm{~s}), 999(\mathrm{~m}), 917(\mathrm{~s}), 822(\mathrm{~s}), 751$ (s).

HRMS(ESI) calcd for $\mathrm{C}_{16} \mathrm{H}_{18} \mathrm{O}_{3}{ }^{+}(\mathrm{M}+\mathrm{H})$ 259.1329, found 259.1335 .

\section{Tosyl azide (74)}

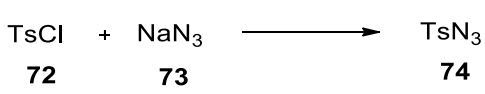

Following a reported procedure, ${ }^{[8]}$ a solution of sodium azide (73) (10.3 g, 157 mmol, 1.50 equiv) in water $(60 \mathrm{~mL})$ was added dropwise over $1 \mathrm{~h}$ to a solution of tosyl chloride (72) (20 g, $0.11 \mathrm{~mol}, 1.0$ equiv) in acetone $(200 \mathrm{~mL})$ at $0^{\circ} \mathrm{C}$. The reaction was allowed to warm up to $23^{\circ} \mathrm{C}$ and stirred for $16 \mathrm{~h}$. The acetone was removed under reduced pressure at $25^{\circ} \mathrm{C}$ and the reaction mixture was extracted with ether (x2). The combined organic layers were washed with water (x2), $5 \% \mathrm{Na}_{2} \mathrm{CO}_{3}(\mathrm{x} 2)$ and water (x2), dried over $\mathrm{MgSO}_{4}$ and the solvent was removed under reduced pressure to afford tosyl azide (74) (20.7 g, $107 \mathrm{mmol}$, quantitative) as a colorless oil which solidified under storage at $4^{\circ} \mathrm{C}$.

${ }^{1} \mathrm{H} \mathrm{NMR}\left(\mathrm{CDCl}_{3}, 400 \mathrm{MHz}\right) \delta 7.87(\mathrm{~d}, J=8.4 \mathrm{~Hz}, 2 \mathrm{H} ; \mathrm{ArH}), 7.43(\mathrm{~d}, J=8.4 \mathrm{~Hz}, 2 \mathrm{H} ; \mathrm{ArH}), 2.51$ (s, $\left.3 \mathrm{H} ; \mathrm{CH}_{3}\right)$.

The characterization data for $\mathbf{7 4}$ corresponded to the reported values. ${ }^{[5]}$

\section{Dimethyl 2-diazomalonate (2)}

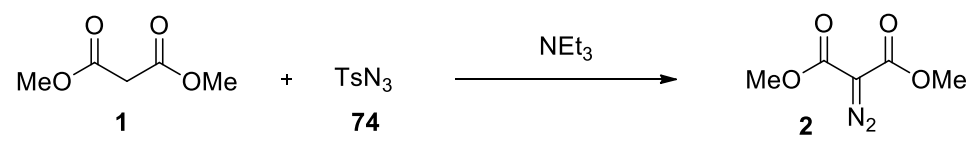

Following a reported procedure, ${ }^{[9]}$ dimethylmalonate $(\mathbf{1})(7.93 \mathrm{~mL}, 69.7 \mathrm{mmol}, 1$ equiv), triethylamine (10.6 mL, $76.6 \mathrm{mmol}, 1.1$ equiv) and tosyl azide (74) (15.1 g, $76.6 \mathrm{mmol}, 1.1$ equiv) were dissolved in acetonitrile $(100 \mathrm{~mL})$. The solution was stirred at $23^{\circ} \mathrm{C}$ for $20 \mathrm{~h}$. The solution was concentrated under reduced pressure and partitioned between DCM and water. The layers were separated and the aqueous layer was extracted with DCM (x1). The organic layers were combined and dried over $\mathrm{MgSO}_{4}$. The crude was first filtered over a plug of silica gel 
$\left(\mathrm{PET}_{2} \mathrm{Et}_{2} \mathrm{O}\right.$ 1/1) to remove most of the tosylamide formed during the reaction. Then, purification by column chromatography ( $\left.\mathrm{PET} / \mathrm{Et}_{2} \mathrm{O} 1 / 1\right)$ afforded dimethyl 2-diazomalonate (2) (10.4 g, 65.5 mmol, 94\%) as a yellow oil which solidified under storage at $4^{\circ} \mathrm{C}$.

$\mathrm{R}_{f} 0.32\left(\mathrm{PET} / \mathrm{Et}_{2} \mathrm{O}\right.$ 1:1, Anisaldehyde).

${ }^{1} \mathrm{H} \mathrm{NMR}\left(\mathrm{CDCl}_{3}, 400 \mathrm{MHz}\right) \delta 3.87\left(\mathrm{~s}, 1 \mathrm{H} ; \mathrm{CH}_{3}\right)$.

The characterization data for 2 corresponded to the reported values. ${ }^{[6]}$

\section{Dimethyl 2-(4-methoxyphenyl)cyclopropane-1,1-dicarboxylate (3b)}

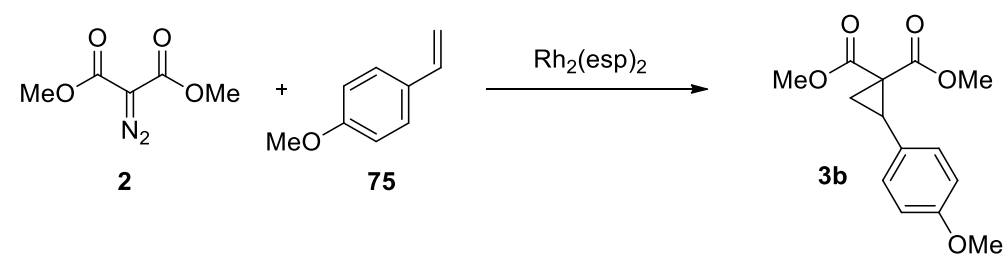

The reaction was performed following GP1, using $\mathrm{Rh}_{2}(\mathrm{esp})_{2}(4.8 \mathrm{mg}, 6.3 \mu \mathrm{mol}, 0.1 \mathrm{~mol} \%)$, styrene (75) (0.85 mL, $6.3 \mathrm{mmol}, 1$ equiv) and diazomalonate (2) (1.0 g, $6.3 \mathrm{mmol}, 1.0$ equiv). Purification by column chromatography (PET/Et $2 \mathrm{O} 5: 1$ to $3: 1)$ afforded cyclopropane $\mathbf{3 b}$ (1.58 g, $5.98 \mathrm{mmol}, 95 \%)$.

$\mathrm{R}_{f} 0.24\left(\mathrm{PET}_{\mathrm{Et}} \mathrm{O}\right.$ 3:1).

${ }^{1} \mathrm{H}$ NMR $\left(\mathrm{CDCl}_{3}, 400 \mathrm{MHz}\right) \delta$ 7.17-7.11 (m, 2H; ArH), 6.86-6.79 (m, 2H; ArH), 3.81 (s, 3H; $\left.\mathrm{CH}_{3}\right), 3.80$ (s, 3H; $\left.\mathrm{CH}_{3}\right), 3.41\left(\mathrm{~s}, 3 \mathrm{H} ; \mathrm{CH}_{3}\right), 3.20$ (t, $\left.J=8.2 \mathrm{~Hz}, 1 \mathrm{H} ; \mathrm{CHPh}\right), 2.18$ (dd, $J=8.1,5.2$ $\left.\mathrm{Hz}, 1 \mathrm{H} ; \mathrm{CH}_{2}\right), 1.74$ (dd, $\left.J=9.3,5.2 \mathrm{~Hz}, 1 \mathrm{H} ; \mathrm{CH}_{2}\right)$.

The characterization data for $\mathbf{3 b}$ corresponded to the reported values. ${ }^{[1]}$

\section{Dimethyl 2-phenylcyclopropane-1,1-dicarboxylate (3c)}

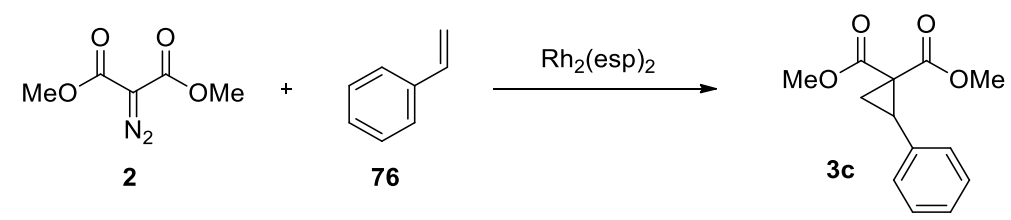

The reaction was performed following GP1, using $\mathrm{Rh}_{2}(\mathrm{esp})_{2}(4.8 \mathrm{mg}, 6.3 \mu \mathrm{mol}, 0.1 \mathrm{~mol} \%$ ), styrene (76) (0.73 mL, $6.3 \mathrm{mmol}, 1$ equiv) and diazomalonate (2) (1.0 g, $6.3 \mathrm{mmol}, 1.0$ equiv). 
Purification by column chromatography (PET/Et $2 \mathrm{O} 5: 1$ to 3:1) afforded cyclopropane 3c (1.43 g, $6.11 \mathrm{mmol}, 97 \%)$.

$\mathrm{R}_{f} 0.45$ (PET/AcOEt 3/1).

${ }^{1} \mathrm{H}$ NMR $\left(\mathrm{CDCl}_{3}, 400 \mathrm{MHz}\right) \delta$ 7.33-7.19 (m, 5H; $\left.\mathrm{ArH}\right), 3.82\left(\mathrm{~s}, 3 \mathrm{H} ; \mathrm{CH}_{3}\right), 3.39\left(\mathrm{~s}, 3 \mathrm{H} ; \mathrm{CH}_{3}\right)$, 3.26 (t, $J=8.8 \mathrm{~Hz}, 1 \mathrm{H} ; \mathrm{CHPh}), 2.23\left(\mathrm{dd}, J=7.9,5.2 \mathrm{~Hz}, 1 \mathrm{H} ; \mathrm{CH}_{2}\right), 1.77$ (dd, $J=9.3,5.2 \mathrm{~Hz}$, $\left.1 \mathrm{H} ; \mathrm{CH}_{2}\right)$.

The characterization data for $\mathbf{3 c}$ corresponded to the reported values. ${ }^{[1]}$

\section{Dimethyl 2-p-tolylcyclopropane-1,1-dicarboxylate (3d)}

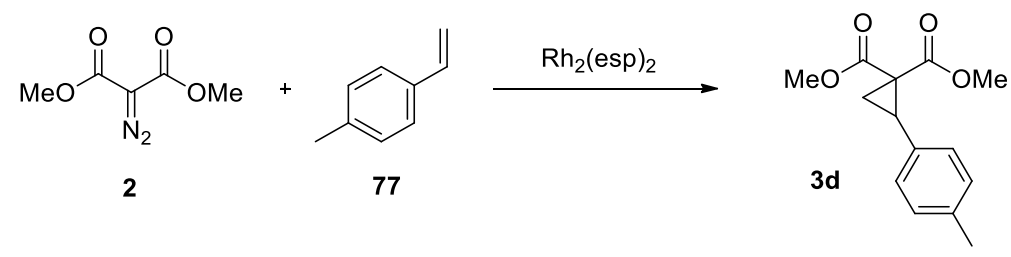

The reaction was performed following the GP1, using $\mathrm{Rh}_{2}(\mathrm{esp})_{2}(4.8 \mathrm{mg}, 6.3 \mu \mathrm{mol}, 0.1 \mathrm{~mol} \%)$, styrene (77) (0.83 mL, $6.3 \mathrm{mmol}, 1.0$ equiv) and diazomalonate (2) (1.0 g, $6.3 \mathrm{mmol}, 1.0$ equiv). Purification by column chromatography (PET/Et $2 \mathrm{O} 5: 1$ to $3: 1)$ afforded cyclopropane $3 \mathbf{d}$ (1.53 g, $6.15 \mathrm{mmol}, 97 \%)$.

$\mathrm{R}_{f} 0.63$ (PET/AcOEt 3/1).

${ }^{1} \mathrm{H}$ NMR $\left(\mathrm{CDCl}_{3}, 400 \mathrm{MHz}\right) \delta 7.10(\mathrm{~m}, 4 \mathrm{H} ; \mathrm{ArH}), 3.81\left(\mathrm{~s}, 3 \mathrm{H} ; \mathrm{OCH}_{3}\right), 3.41\left(\mathrm{~s}, 3 \mathrm{H} ; \mathrm{OCH}_{3}\right), 3.22$ $(\mathrm{t}, J=8.9 \mathrm{~Hz}, 1 \mathrm{H} ; \mathrm{CHPh}), 2.33\left(\mathrm{~s}, 3 \mathrm{H} ; \mathrm{CH}_{3}\right), 2.20\left(\mathrm{dd}, J=8.1,5.2 \mathrm{~Hz}, 1 \mathrm{H} ; \mathrm{CH}_{2}\right), 1.75(\mathrm{dd}, J=$ 9.3, 5.2 Hz, $1 \mathrm{H} ; \mathrm{CH}_{2}$ ).

Dimethyl 2-(4-chlorophenyl)cyclopropane-1,1-dicarboxylate (3e)

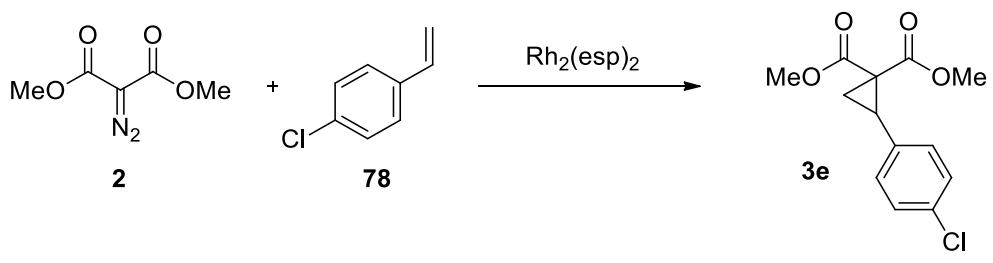

The reaction was performed following the GP1, using $\mathrm{Rh}_{2}(\mathrm{esp})_{2}(4.8 \mathrm{mg}, 6.3 \mu \mathrm{mol}, 0.1 \mathrm{~mol} \%$ ), styrene (78) $(0.76 \mathrm{~mL}, 6.3 \mathrm{mmol}, 1.00$ equiv) and diazomalonate (2) (1.0 g, $6.3 \mathrm{mmol}, 1.0$ 
equiv). Purification by column chromatography ( $\mathrm{PET}_{\mathrm{Et}} \mathrm{O}$ 5:1 to 3:1) afforded cyclopropane 3e (1.69 g, $6.27 \mathrm{mmol}, 99 \%)$.

$\mathrm{R}_{f} 0.63$ (PET/AcOEt 3:1).

${ }^{1} \mathrm{H}$ NMR $\left(\mathrm{CDCl}_{3}, 400 \mathrm{MHz}\right) \delta 7.27(\mathrm{~d}, J=8.6 \mathrm{~Hz}, 2 \mathrm{H} ; \mathrm{ArH}), 7.15(\mathrm{~d}, J=8.4 \mathrm{~Hz}, 2 \mathrm{H}$; ArH), 3.82 (s, 3H; $\left.\mathrm{CH}_{3}\right), 3.43$ (s, 3H; $\left.\mathrm{CH}_{3}\right), 3.21$ (t, $\left.J=8.6 \mathrm{~Hz}, 1 \mathrm{H} ; \mathrm{CHPh}\right), 2.18$ (dd, $J=8.1,5.3 \mathrm{~Hz}, 1 \mathrm{H}$; $\left.\mathrm{CH}_{2}\right), 1.77\left(\mathrm{dd}, J=9.3,5.3 \mathrm{~Hz}, 1 \mathrm{H} ; \mathrm{CH}_{2}\right)$.

The characterization data for $\mathbf{3 e}$ corresponded to the reported values. ${ }^{[1]}$

Dimethyl 2-phenethylcyclopropane-1,1-dicarboxylate (3f)

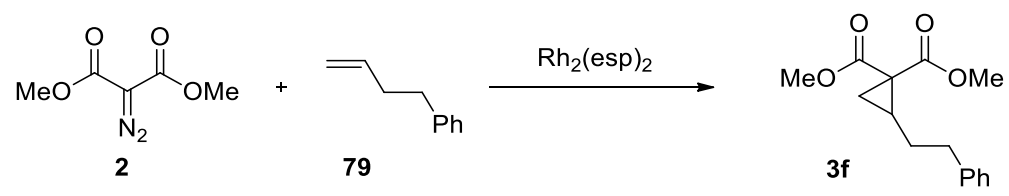

The reaction was performed following the GP1, using $\mathrm{Rh}_{2}(\mathrm{esp})_{2}(2.4 \mathrm{mg}, 3.1 \mu \mathrm{mol}, 0.1 \mathrm{~mol} \%)$, 4-phenylbutene (79) (0.48 mL, $3.2 \mathrm{mmol}, 1.0$ equiv) and diazomalonate (2) (0.50 g, $3.2 \mathrm{mmol}$, 1.0 equiv). Purification by column chromatography ( $\mathrm{PET}_{2} \mathrm{Et}_{2} \mathrm{O} 5: 1$ to $3: 1$ ) afforded cyclopropane 3f $(0.77 \mathrm{~g}, 2.9 \mathrm{mmol}, 93 \%)$.

$\mathrm{R}_{f} 0.55$ (PET/AcOEt 3:1).

${ }^{1} \mathrm{H}$ NMR $\left(\mathrm{CDCl}_{3}, 400 \mathrm{MHz}\right) \delta$ 7.34-7.25 (m, 2H; ArH), 7.24-7.13 (m, 3H; ArH), 3.79 (s, 3H; $\left.\mathrm{CH}_{3}\right), 3.75\left(\mathrm{~s}, 3 \mathrm{H} ; \mathrm{CH}_{3}\right), 2.75\left(\mathrm{~m}, 2 \mathrm{H} ; \mathrm{PhCH}_{2}\right), 1.97(\mathrm{~m}, 1 \mathrm{H}$, cyclopropaneH $), 1.79$ (m, 1H; cyclopropaneH), 1.55 (m, 1H; cyclopropaneH), 1.42 (m, 2H; $\mathrm{CH}_{2} \mathrm{CH}_{2}$ ).

The characterization data for $\mathbf{3 f}$ corresponded to the reported values. ${ }^{[1]}$

\section{Methyl}

1-(3,4-dihydro-2H-pyran-6-carbonyl)-2-(4-

methoxyphenyl)cyclopropanecarboxylate (6b) (trans/cis)

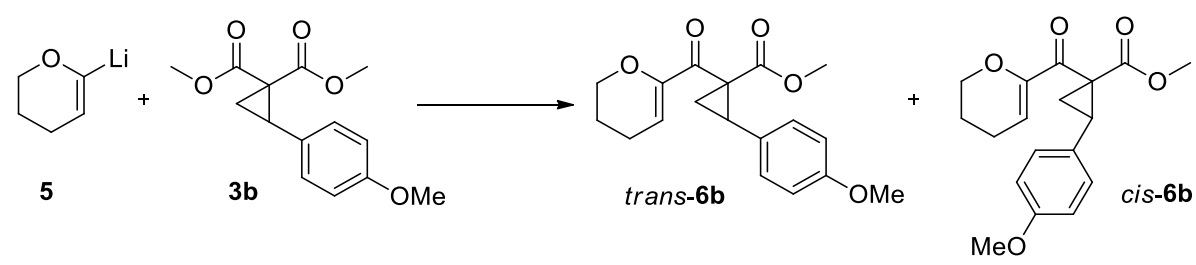


Following a reported procedure, ${ }^{[10]}$ dihydropyran (44) $(52 \mu \mathrm{L}, 0.57 \mathrm{mmol}, 1.0$ equiv) was dissolved in THF $(0.13 \mathrm{~mL})$ and the mixture was cooled to $-78^{\circ} \mathrm{C}$. ${ }^{\mathrm{t}} \mathrm{BuLi}(1.6 \mathrm{M}$ solution in pentane, $0.36 \mathrm{~mL}, 0.57 \mathrm{mmol}, 1$ equiv) was added dropwise to this solution at the same temperature. The reaction mixture was stirred at $0^{\circ} \mathrm{C}$ for $30 \mathrm{~min}$. Then THF $(1.5 \mathrm{~mL})$ was added to 5 and the solution was cooled to $-95^{\circ} \mathrm{C}$. The 1,1-cyclopropane diester $\mathbf{3 b}(0.15 \mathrm{~g}, 0.57 \mathrm{mmol}, 1$ equiv) dissolved in THF $(0.5 \mathrm{~mL})$ was added in one portion to this solution. The reaction mixture was allowed to stir for $2 \mathrm{~h}$ until the temperature of the cooling bath was $-40^{\circ} \mathrm{C}$. Then the reaction mixture was poured in brine and diluted with DCM. The aqueous layer was extracted with DCM (x3). The organic layers were combined, dried over $\mathrm{Na}_{2} \mathrm{SO}_{4}$ and evaporated under reduced pressure. A 3/1 mixture of trans/cis is detected by ${ }^{1} \mathrm{H}$ NMR of the crude. Purification by column chromatography (PET/Et $2 \mathrm{O} 5: 1$ to 3:1) afforded the trans isomer of $\mathbf{6 b}(52 \mathrm{mg}, 0.16 \mathrm{mmol}$, $29 \%)$, a 2.3/1 mixture of cis/trans isomers of $\mathbf{6 b}(27 \mathrm{mg}, 85 \mu \mathrm{mol}, 15 \%)$ and starting material $\mathbf{3 b}$ (33 mg, $0.12 \mathrm{mmol}, 21 \%)$.

\section{Trans isomer}

$\mathrm{R}_{f} 0.41$ (PET/AcOEt 3:1).

${ }^{1} \mathrm{H}$ NMR $\left(\mathrm{CDCl}_{3}, 400 \mathrm{MHz}\right) \delta$ 7.20-7.15 (m, 2H; ArH), 6.85-6.80 (m, 2H; ArH), 6.02 (t, J = 4.1 $\mathrm{Hz}, 1 \mathrm{H}$; alkeneH), 4.18-4.11 (m, 1H; $\left.\mathrm{OCH}_{2}\right), 3.95-4.01$ (m, $\left.1 \mathrm{H} ; \mathrm{OCH}_{2}\right), 3.80$ (s, 3H; $\left.\mathrm{OCH}_{3}\right)$, 3.43 (s, 3H; $\left.\mathrm{OCH}_{3}\right), 3.40$ (t, $\left.J=8.6 \mathrm{~Hz}, 1 \mathrm{H} ; \mathrm{CHPh}\right), 2.29-2.19\left(\mathrm{~m}, 3 \mathrm{H} ; \mathrm{CH}_{2}\right), 1.96-1.84$ (m, 2H; $\left.\mathrm{CH}_{2}\right), 1.51\left(\mathrm{dd}, J_{1}=9.3,4.8 \mathrm{~Hz}, 1 \mathrm{H} ; \mathrm{CH}_{2}\right)$.

${ }^{13} \mathrm{C}$ NMR $\left(\mathrm{CDCl}_{3} 100 \mathrm{MHz}\right) \delta 190.8,168.5,158.7,150.8,130.05,126.9,113.5,109.9,66.3$, 55.2, 52.0, 41.3, 30.0, 21.8, 20.6, 20.6.

IR $v\left(\mathrm{~cm}^{-1}\right)$ : $2951(\mathrm{w}), 2838(\mathrm{w}), 2359(\mathrm{w}), 1741(\mathrm{~m}), 1689(\mathrm{~m}), 1630(\mathrm{~m}), 1517(\mathrm{~s}), 1438(\mathrm{~m})$, 1321 (m), 1291 (s), 1251 (s), 1218 (m), 1175 (m), 1147 (m), 1057 (m), 1036 (m), 921 (m), 838 (m), 749 (s).

HRMS (ESI) calcd for $\mathrm{C}_{18} \mathrm{H}_{21} \mathrm{O}_{5}{ }^{+}(\mathrm{M}+\mathrm{H}) 317.1389$; found 317.1395.

Cis isomer

$\mathrm{R}_{f} 0.42$ (PET/AcOEt 3/1).

[10] Boeckman, R. K.; Bruza, K. J. Tetrahedron 1981, 37, 3997. 
${ }^{1} \mathrm{H}$ NMR $\left(\mathrm{CDCl}_{3}, 400 \mathrm{MHz}\right) \delta$ 7.10-7.03 (m, 2H; ArH), 6.79-6.74 (m, 2H; ArH), 5.73 (t, J = 4.3 $\mathrm{Hz}, 1 \mathrm{H}$; alkeneH), $3.93\left(\mathrm{t}, J=5.0 \mathrm{~Hz}, 2 \mathrm{H} ; \mathrm{OCH}_{2}\right), 3.77\left(\mathrm{~s}, 3 \mathrm{H} ; \mathrm{OCH}_{3}\right), 3.76\left(\mathrm{~s}, 3 \mathrm{H} ; \mathrm{OCH}_{3}\right), 3.34$ (t, $J=8.4 \mathrm{~Hz}, 1 \mathrm{H} ; \mathrm{CHPh}), 2.26-2.21\left(\mathrm{~m}, 1 \mathrm{H} ; \mathrm{CH}_{2}\right), 2.08-2.02\left(\mathrm{~m}, 2 \mathrm{H} ; \mathrm{CH}_{2}\right), 1.77-1.71$ (m, 2H; $\left.\mathrm{CH}_{2}\right), 1.57$ (dd, $\left.J=9.5,5.3 \mathrm{~Hz}, 1 \mathrm{H} ; \mathrm{CH}_{2}\right)$.

\section{Methyl 1-(3,4-dihydro-2H-pyran-6-carbonyl)-2-phenylcyclopropanecarboxylate (6c)}

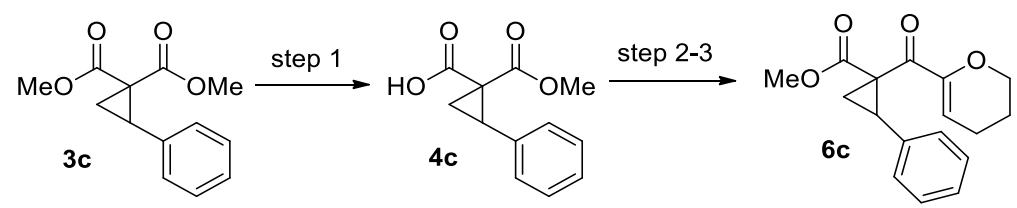

Following GP2, 1,1-cyclopropane diester 3c (555 mg, $2.37 \mathrm{mmol}, 1.00$ equiv) was dissolved in $\mathrm{MeOH}(1.7 \mathrm{~mL}), 1.7 \mathrm{~N}$ aqueous $\mathrm{NaOH}(1.7 \mathrm{~mL}, 2.8 \mathrm{mmol}, 1.2$ equiv) and some drops of THF. The reaction afforded acid $\mathbf{4 c}$ (503 $\mathrm{mg}, 2.28 \mathrm{mmol}, 96 \%)$.

${ }^{1} \mathrm{H} \mathrm{NMR}\left(\mathrm{CDCl}_{3}, 400 \mathrm{MHz}\right) \delta 12.01$ (brs, $\left.1 \mathrm{H} ; \mathrm{COOH}\right), 7.36-7.19(\mathrm{~m}, 5 \mathrm{H} ; \mathrm{ArH}), 3.41$ (t, $J=8.9$ $\mathrm{Hz}, 1 \mathrm{H} ; \mathrm{CHPh}), 3.29$ (s, 3H; $\mathrm{OCH}_{3}$ ), 2.42 (dd, $\left.J=8.6,4.8 \mathrm{~Hz}, 1 \mathrm{H} ; \mathrm{CH}_{2}\right), 2.27$ (dd, $J=9.3,4.8$ $\left.\mathrm{Hz}, 1 \mathrm{H} ; \mathrm{CH}_{2}\right)$.

Following GP3, dihydropyran (44) (0.46 mL, 5.0 mmol, 2.2 equiv) was dissolved in THF (1 mL) and reacted with ${ }^{t} \mathrm{BuLi}$ (1.6 $\mathrm{M}$ solution in pentane, $3.14 \mathrm{~mL}, 5.02 \mathrm{mmol}, 2.20$ equiv). The solution was diluted with THF (15 mL) and was added to acid 4c (503 mg, $2.28 \mathrm{mmol}, 1.00$ equiv) in THF (6 mL). The obtained oil was dissolved in DMF (21 mL). $\mathrm{K}_{2} \mathrm{CO}_{3}(630 \mathrm{mg}, 4.55$ mmol, 2.00 equiv) and methyl iodide (710 $\mu 1,11.4 \mathrm{mmol}, 5.00$ equiv) were added. The crude product was purified by column chromatography $\left(\mathrm{PET}_{/ 2} \mathrm{Et}_{2} \mathrm{O} 8: 1\right.$ to $\left.3: 1\right)$ to afford the desired product 6c (350 mg, $1.22 \mathrm{mmol}, 54 \%(70 \% \mathrm{brsm}))$ as a colourless oil and 1,1-cyclopropane diester $3 \mathbf{c}(0.13 \mathrm{~g}, 0.55 \mathrm{mmol}, 23 \% \mathrm{rsm})$.

$\mathrm{R}_{f} 0.50$ (PET/AcOEt 3:1).

${ }^{1} \mathrm{H} \mathrm{NMR}\left(\mathrm{CDCl}_{3}, 400 \mathrm{MHz}\right) \delta$ 7.27-7.12 (m, 5H; ArH), $5.71(\mathrm{t}, J=4.5 \mathrm{~Hz}, 1 \mathrm{H}$; alkeneH), 3.90 (dt, $\left.J=7.0,4.5 \mathrm{~Hz}, 2 \mathrm{H} ; \mathrm{OCH}_{2}\right), 3.77$ (s, 3H; $\left.\mathrm{OCH}_{3}\right), 3.37$ (t, $\left.J=8.5 \mathrm{~Hz}, 1 \mathrm{H} ; \mathrm{CHPh}\right), 2.27$ (dd, $J$ $=8.0,5.0 \mathrm{~Hz}, 1 \mathrm{H}$; cyclopropaneH), 2.11-1.92 (m, 2H; $\left.\mathrm{CH}_{2} \mathrm{CH}_{2}\right), 1.74-1.64\left(\mathrm{~m}, 2 \mathrm{H} ; \mathrm{CH}_{2} \mathrm{CH}_{2}\right)$, 1.61 (dd, $J=9.0,5.0 \mathrm{~Hz}, 1 \mathrm{H}$; cyclopropaneH).

${ }^{13} \mathrm{C} \mathrm{NMR}\left(\mathrm{CDCl}_{3}, 100 \mathrm{MHz}\right) \delta 188.8,171.3,150.7,134.2,128.4,128.0,127.0,110.1,66.1,52.5$, 40.4, 32.7, 21.6, 20.5, 17.2. 
IR v (cm-1): 3333 (w), $2951(\mathrm{w}), 2867$ (w), 2147 (w), 1734 (s), 1698 (m), 1631 (s), 1500 (w), 1436 (m), 1320 (m), 1277 (s), 1212 (m), 1150 (s), 1060 (s), 922 (w), 905 (w), 767 (m), 739 (w), $698(\mathrm{~m})$.

HRMS (ESI) calcd for $\mathrm{C}_{17} \mathrm{H}_{19} \mathrm{O}_{4}{ }^{+}(\mathrm{M}+\mathrm{H}) 287.1283$; found 287.1285.

\section{Methyl 1-(3,4-dihydro-2H-pyran-6-carbonyl)-2-p-tolylcyclopropanecarboxylate (6d)}

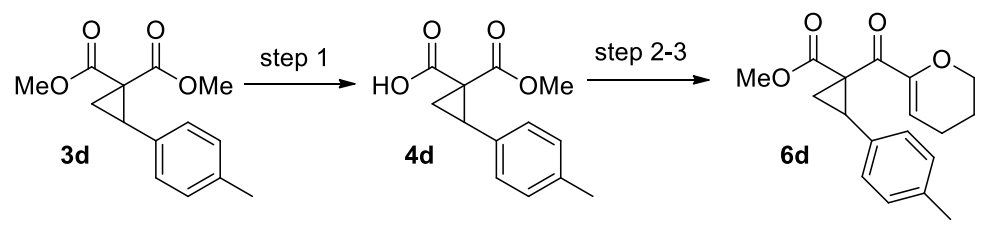

Following GP2, 1,1-cyclopropane diester 3d (718 mg, $2.89 \mathrm{mmol}, 1.00$ equiv) was dissolved in $\mathrm{MeOH}(2.0 \mathrm{~mL}), 1.7 \mathrm{~N}$ aqueous $\mathrm{NaOH}(2.0 \mathrm{~mL}, 3.4 \mathrm{mmol}, 1.2$ equiv) and some drops of THF. The reaction afforded acid $\mathbf{4 d}$ (629 $\mathrm{mg}, 2.69 \mathrm{mmol}, 93 \%)$.

${ }^{1} \mathrm{H}$ NMR (400 MHz, $\left.\mathrm{CDCl}_{3}\right) \delta: 7.14(\mathrm{~m}, 4 \mathrm{H} ; \mathrm{ArH}), 3.40$ (t, $\left.J=9.1 \mathrm{~Hz}, 1 \mathrm{H} ; \mathrm{CHPh}\right), 3.31$ (s, 3H; $\mathrm{OCH}_{3}$ ), 2.42 (ddd, $\left.J=8.6,4.6,1.2 \mathrm{~Hz}, 1 \mathrm{H} ; \mathrm{CH}_{2}\right), 2.37-2.31\left(\mathrm{~m}, 1 \mathrm{H} ; \mathrm{CH}_{2}\right), 2.37$ (s, 3H; $\left.\mathrm{CH}_{3}\right)$.

Following GP3, dihydropyran (44) $(540 \mu \mathrm{L}, 5.92 \mathrm{mmol}, 2.20$ equiv) was dissolved in THF (1.25 $\mathrm{mL}$ ) and reacted with ${ }^{t} \mathrm{BuLi}(1.6 \mathrm{M}$ solution in pentane, $3.70 \mathrm{~mL}, 5.92 \mathrm{mmol}, 2.20$ equiv). The solution was diluted with THF (15 mL) and added to acid 4d (629 mg, $2.69 \mathrm{mmol}, 1.00$ equiv) in THF (8 mL). The obtained oil was dissolved in DMF (25 ml). $\mathrm{K}_{2} \mathrm{CO}_{3}(740 \mathrm{mg}, 5.36 \mathrm{mmol}, 2.00$ equiv) and methyl iodide ( $0.83 \mathrm{~mL}, 13.4 \mathrm{mmol}, 5.00$ equiv) were added. The crude product was purified by column chromatography $\left(\mathrm{PET} \mathrm{Et}_{2} \mathrm{O} 8: 1\right.$ to 3:1) to afford the desired product $\mathbf{6 d}$ (562 $\mathrm{mg}, 1.87 \mathrm{mmol}, 70 \%$ (86\% brsm)) as a colourless oil and the 1,1-cyclopropane diester $\mathbf{3 d}(0.13$ $\mathrm{mg}, 0.51 \mathrm{mmol}, 0.18 \mathrm{rsm})$.

$\mathrm{R}_{f} 0.50$ (PET/AcOEt 3:1).

${ }^{1} \mathrm{H}$ NMR $\left(\mathrm{CDCl}_{3}, 400 \mathrm{MHz}\right) \delta 7.02(\mathrm{~m}, 4 \mathrm{H} ; \mathrm{ArH}), 5.72(\mathrm{t}, J=4.3 \mathrm{~Hz}, 1 \mathrm{H}$; alkeneH), $3.92(\mathrm{t}, J=$ $\left.5.2 \mathrm{~Hz}, 2 \mathrm{H} ; \mathrm{OCH}_{2}\right), 3.76$ (s, 3H; $\left.\mathrm{OCH}_{3}\right), 3.35$ (t, $\left.J=8.6 \mathrm{~Hz}, 1 \mathrm{H} ; \mathrm{CHPh}\right), 2.29$ (s, 3H; $\left.\mathrm{CH}_{3}\right), 2.25$ (dd, $J=7.9,5.0 \mathrm{~Hz}, 1 \mathrm{H}$; cyclopropaneH), 2.08-1.99 (m, 2H; $\left.\mathrm{CH}_{2} \mathrm{CH}_{2}\right), 1.75-1.67$ (m, 2H; $\left.\mathrm{CH}_{2} \mathrm{CH}_{2}\right), 1.58$ (dd, $J=9.1,5.2 \mathrm{~Hz}, 1 \mathrm{H}$; cyclopropaneH).

${ }^{13} \mathrm{C} \mathrm{NMR}\left(\mathrm{CDCl}_{3}, 100 \mathrm{MHz}\right) \delta 188.9,171.4,150.8,136.6,131.0,128.7,128.4,109.9,66.1,52.4$, 40.3, 32.6, 21.6, 21.1, 20.5, 17.3. 
IR $v\left(\mathrm{~cm}^{-1}\right): 3483(\mathrm{w}), 2953(\mathrm{w}), 1733(\mathrm{~s}), 1631(\mathrm{~m}), 1519(\mathrm{~m}), 1437(\mathrm{~m}), 1376(\mathrm{~m}), 1320(\mathrm{~m})$, 1276 (s), 1210 (m), 1150 (s), 1060 (s), 923 (w), $826(w), 738(w)$.

HRMS (ESI) calcd for $\mathrm{C}_{18} \mathrm{H}_{21} \mathrm{O}_{4}{ }^{+}(\mathrm{M}+\mathrm{H}) 301.1440$; found 301.1450 .

Methyl 2-(4-chlorophenyl)-1-(3,4-dihydro-2H-pyran-6-carbonyl)cyclopropanecarboxylate (6e)

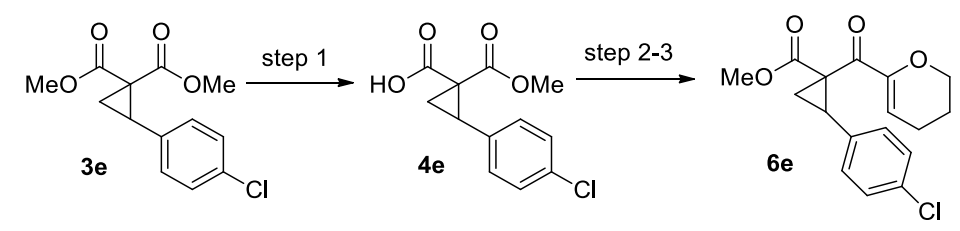

Following GP2, 1,1-cyclopropane diester 3c (607 mg, $2.26 \mathrm{mmol}, 1.00$ equiv) was dissolved in $\mathrm{MeOH}(1.6 \mathrm{~mL}), 1.7 \mathrm{~N}$ aqueous $\mathrm{NaOH}(1.6 \mathrm{ml}, 2.7 \mathrm{mmol}, 1.2$ equiv) and some drops of THF. The reaction afforded acid $\mathbf{4 e}(527 \mathrm{mg}, 2.07 \mathrm{mmol}, 92 \%)$.

${ }^{1} \mathrm{H}$ NMR $\left(\mathrm{CDCl}_{3}, 400 \mathrm{MHz}\right) \delta 7.31(\mathrm{~d}, J=8.4 \mathrm{~Hz}, 2 \mathrm{H} ; \mathrm{ArH}), 7.20(\mathrm{~d}, J=8.4 \mathrm{~Hz}, 2 \mathrm{H}$; ArH), 3.41-3.33 (m, 4H; CHPh and $\left.\mathrm{CH}_{3}\right), 2.38\left(\mathrm{dd}, J=8.6,5.0 \mathrm{~Hz}, 1 \mathrm{H} ; \mathrm{CH}_{2}\right), 2.28(\mathrm{dd}, J=9.3,5.0 \mathrm{~Hz}$, $\left.1 \mathrm{H} ; \mathrm{CH}_{2}\right)$.

Following GP3, dihydropyran (44) $(420 \mu \mathrm{L}, 4.55 \mathrm{mmol}, 2.20$ equiv) was dissolved in THF (1 $\mathrm{mL}$ ) and reacted with ${ }^{t} \mathrm{BuLi}$ (1.6 M solution in pentane, $2.85 \mathrm{~mL}, 4.55 \mathrm{mmol}, 2.20$ equiv). The solution was added to acid $4 \mathbf{e}(527 \mathrm{mg}, 2.07 \mathrm{mmol}, 1.00$ equiv) in THF (8 mL). The obtained oil was dissolved in DMF (20 mL). $\mathrm{K}_{2} \mathrm{CO}_{3}(570 \mathrm{mg}, 4.14 \mathrm{mmol}, 2.00$ equiv) and methyl iodide (0.65 mL, $10 \mathrm{mmol}, 5.0$ equiv) were added. The crude product was purified by column chromatography (PET/Et $2 \mathrm{O} 8: 1$ to 3:1) to afford the desired product 6e (413 $\mathrm{mg}, 1.29 \mathrm{mmol}$, $62 \%(87 \% \mathrm{brsm})$ as a colourless oil and the 1,1-cyclopropane diester $3 \mathbf{e}(0.16 \mathrm{~g}, 0.58 \mathrm{mmol}, 26 \%$ rsm).

$\mathrm{R}_{f} 0.50$ (PET/AcOEt 3:1).

${ }^{1} \mathrm{H}$ NMR $\left(\mathrm{CDCl}_{3}, 400 \mathrm{MHz}\right) \delta 7.20(\mathrm{~d}, J=8.4 \mathrm{~Hz}, 2 \mathrm{H} ; \mathrm{ArH}), 7.06(\mathrm{~d}, J=8.4 \mathrm{~Hz}, 2 \mathrm{H}$; ArH), 5.74 (t, $J=4.3 \mathrm{~Hz}, 1 \mathrm{H}$; alkeneH), $3.92\left(\mathrm{t}, J=5.5 \mathrm{~Hz}, 2 \mathrm{H} ; \mathrm{OCH}_{2}\right), 3.77$ (s, $\left.3 \mathrm{H} ; \mathrm{OCH}_{3}\right), 3.33$ (t, $J=8.4$ $\mathrm{Hz}, 1 \mathrm{H} ; \mathrm{CHPh}), 2.23$ (dd, $J=7.9,5.2 \mathrm{~Hz}, 1 \mathrm{H}$; cyclopropaneH), 2.11-2.01 (m, 2H; $\mathrm{CH}_{2} \mathrm{CH}_{2}$ ), 1.77-1.68 (m, 2H; $\left.\mathrm{CH}_{2} \mathrm{CH}_{2}\right), 1.59$ (dd, $J=8.9,5.2 \mathrm{~Hz}, 1 \mathrm{H}$; cyclopropaneH). 
${ }^{13} \mathrm{C} \mathrm{NMR}\left(\mathrm{CDCl}_{3}, 100 \mathrm{MHz}\right) \delta 188.6,171.1,150.7,132.9,132.8,129.9,128.2,110.1,66.2,52.5$, 40.5, 31.0, 21.6, 20.5, 17.2.

IR $v\left(\mathrm{~cm}^{-1}\right): 2956(\mathrm{w}), 1736(\mathrm{~s}), 1698(\mathrm{~m}), 1630(\mathrm{~s}), 1497(\mathrm{~m}), 1437(\mathrm{~m}), 1285$ (s), 1151 (s), 1059 (s), $1017(\mathrm{~m}), 922(\mathrm{~m}), 836(\mathrm{~m}), 718(\mathrm{~m})$.

HRMS (ESI) calcd for $\mathrm{C}_{17} \mathrm{H}_{18} \mathrm{ClO}_{4}{ }^{+}(\mathrm{M}+\mathrm{H})$ 321.0894; found 321.0898 .

\section{Methyl 1-(3,4-dihydro-2H-pyran-6-carbonyl)-2-phenethylcyclopropanecarboxylate (6f)}

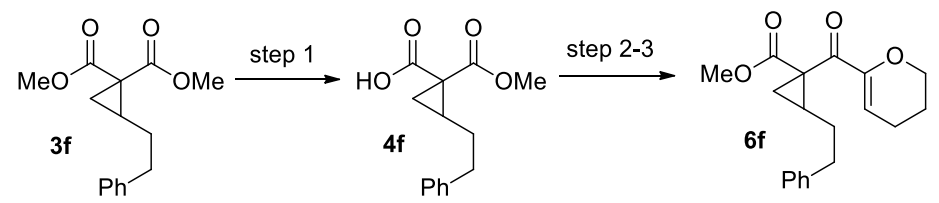

Following GP2, 1,1-cyclopropane diester 3f (440 mg, $1.68 \mathrm{mmol}, 1.00$ equiv) was dissolved in $\mathrm{MeOH}(1.2 \mathrm{~mL}), 1.7 \mathrm{~N}$ aqueous $\mathrm{NaOH}$ (1.2 mL, $2.0 \mathrm{mmol}, 1.2$ equiv) and some drops of THF. The reaction afforded acid $\mathbf{4 f}$ (409 $\mathrm{mg}, 1.64 \mathrm{mmol}, 98 \%)$.

${ }^{1} \mathrm{H}$ NMR $\left(\mathrm{CDCl}_{3}, 400 \mathrm{MHz}\right) \delta$ 7.36-7.11 (m, 5H; $\left.\mathrm{ArH}\right), 3.78\left(\mathrm{~s}, 3 \mathrm{H} ; \mathrm{OCH}_{3}\right), 2.73(\mathrm{~m}, 2 \mathrm{H}$; $\left.\mathrm{CH}_{2} \mathrm{Ph}\right), 2.16\left(\mathrm{~m}, 1 \mathrm{H} ; \mathrm{CH}_{2}\right), 2.07-1.82\left(\mathrm{~m}, 3 \mathrm{H} ; \mathrm{CH}_{2}\right), 1.69(\mathrm{dd}, J=8.4,4.3 \mathrm{~Hz}, 1 \mathrm{H} ; \mathrm{CH})$.

Following GP3, dihydropyran (44) $(330 \mu \mathrm{L}, 3.57 \mathrm{mmol}, 2.20$ equiv) was dissolved in THF (0.75 $\mathrm{mL}$ ) and reacted with ${ }^{t} \mathrm{BuLi}$ (1.6 M solution in pentane, $2.23 \mathrm{~mL}, 3.57 \mathrm{mmol}, 2.20$ equiv). The solution was diluted with THF (10 mL) and added to acid $\mathbf{4 f}$ (409 mg, $1.65 \mathrm{mmol}, 1.00$ equiv) in THF (8 mL). The obtained oil was dissolved in DMF (16 mL). $\mathrm{K}_{2} \mathrm{CO}_{3}$ (455 mg, $3.30 \mathrm{mmol}, 2.00$ equiv) and methyl iodide $(0.51 \mathrm{~mL}, 8.3 \mathrm{mmol}, 5.0$ equiv) were added. The crude product was purified by column chromatography ( $\mathrm{PET}_{2} \mathrm{Et}_{2} \mathrm{O}$ 8:1 to 3:1) to afford of the desired product $\mathbf{6 f}$ (0.29 g, $0.94 \mathrm{mmol}, 57 \%$ (84\% brsm) as a colourless oil and the 1,1-cyclopropane diester $\mathbf{3 f}$ (0.14 mg, $0.53 \mathrm{mmol}, 32 \% \mathrm{rsm})$.

$\mathrm{R}_{f} 0.50$ (PET/AcOEt 3/1).

${ }^{1} \mathrm{H}$ NMR $\left(\mathrm{CDCl}_{3}, 400 \mathrm{MHz}\right) \delta$ 7.32-7.26 (m, 2H; ArH), 7.23-7.14 (m, 3H; ArH), $6.02(\mathrm{t}, J=4.3$ $\mathrm{Hz}, 1 \mathrm{H}$; alkeneH), 4.17-4.10 (m, 1H; $\left.\mathrm{OCH}_{2}\right), 4.04-3.97\left(\mathrm{~m}, 1 \mathrm{H} ; \mathrm{OCH}_{2}\right), 3.72\left(\mathrm{~s}, 3 \mathrm{H} ; \mathrm{OCH}_{3}\right)$, 2.74-2.65 (m, 2H; $\mathrm{CH}_{2} \mathrm{Ph}$ ), 2.28-2.19 (m, 2H; $\left.\mathrm{CH}_{2}\right), 2.19-2.10\left(\mathrm{~m}, 1 \mathrm{H} ; \mathrm{CH}_{2}\right), 1.92-1.82$ (m, 2H; $\mathrm{CH}_{2}$ ), 1.75-1.65 (m, 1H; $\mathrm{CH}_{2}$ ), 1.49 (dd, $J=7.6,4.5 \mathrm{~Hz}, 1 \mathrm{H}$; cyclopropaneH), 1.37-1.28 (m, 1H; $\left.\mathrm{CH}_{2}\right), 1.25$ (dd, $J=8.8,4.3 \mathrm{~Hz}, 1 \mathrm{H}$; cyclopropaneH). 


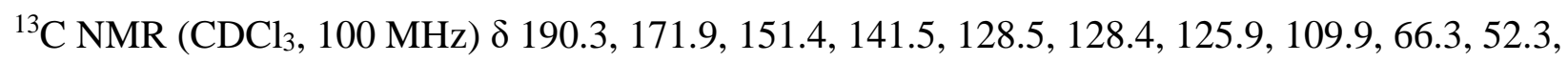
$37.3,35.3,30.0,28.7,21.8,20.7,19.5$.

IR $v\left(\mathrm{~cm}^{-1}\right): 2950(\mathrm{w}), 1736(\mathrm{~s}), 1693(\mathrm{~m}), 1630(\mathrm{~s}), 1436(\mathrm{~m}), 1288(\mathrm{~s}), 1205(\mathrm{~m}), 1153(\mathrm{~m})$, $1058(\mathrm{~m}), 919$ (s), 748 (s), $700(\mathrm{~m})$.

HRMS (ESI) calcd for $\mathrm{C}_{19} \mathrm{H}_{23} \mathrm{O}_{4}{ }^{+}(\mathrm{M}+\mathrm{H})$ 315.1596; found 315.1608 .

\section{Cyclization of ketoesters}

Table 1. Optimization of the homo-Nazarov cyclization for ketoester substrates

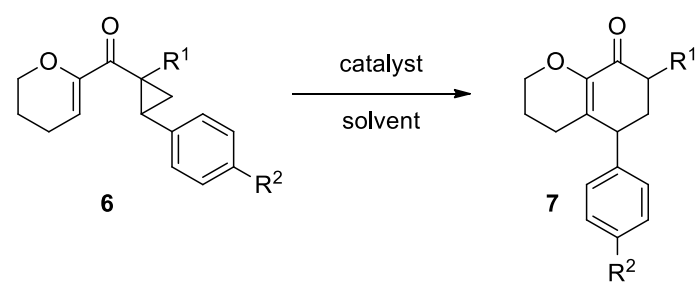

Cyclopropane 6c $(20 \mathrm{mg}, 70 \mu \mathrm{mol})$ was dissolved on the indicated solvent $(0.05 \mathrm{M})$. The indicated catalyst was added and the solution was stirred at the indicated temperature during the indicated time. The reaction was quenched with a saturated solution of $\mathrm{NaHCO}_{3}$, extracted with $\mathrm{Et}_{2} \mathrm{O}(3 \mathrm{x})$, dried over $\mathrm{Na}_{2} \mathrm{SO}_{4}$ and concentrated on reduced pressure.

Cyclopropane $6 \mathbf{a}$ and $\mathbf{6 b}$ were used at the following scale and concentration:

Entry $1\left(\mathbf{R}^{1}=\mathbf{H} ; \mathbf{R}^{2}=\mathbf{M e O}\right): \mathbf{6 a}(103 \mathrm{mg}, 0.399 \mathrm{mmol}) 0.04 \mathrm{M}$ in $\mathrm{CH}_{3} \mathrm{CN}$.

Entry $2\left(\mathbf{R}^{1}=\mathbf{C O} 2 \mathbf{M e} ; \mathbf{R}^{2}=\mathbf{M e O}\right): 6 \mathbf{b}(17 \mathrm{mg}, 54 \mu \mathrm{mol}) 0.05 \mathrm{M}$ in $\mathrm{CH}_{3} \mathrm{CN}$.

Entry $15\left(\mathbf{R}^{\mathbf{1}}=\mathbf{H} ; \mathbf{R}^{\mathbf{2}}=\mathbf{M e O}\right)$ : $6 \mathbf{a}(10 \mathrm{mg}, 39 \mu \mathrm{mol}) 0.04 \mathrm{M}$ in $\mathrm{CH}_{2} \mathrm{Cl}_{2}$.

5-(4-Methoxyphenyl)-3,4,6,7-tetrahydro-2H-chromen-8(5H)-one (7a)

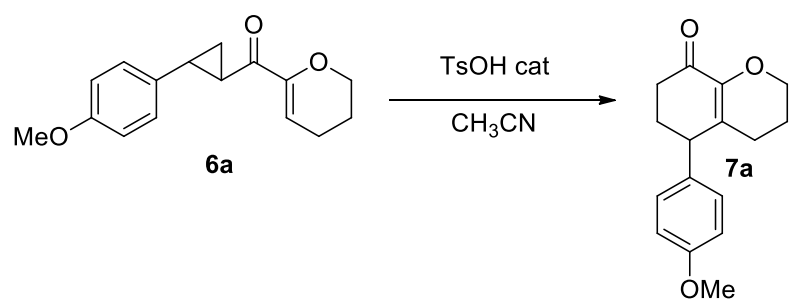

The reaction was performed following GP4, starting from cyclopropane 6a (100 $\mathrm{mg}, 0.40 \mathrm{mmol}$, 1.0 equiv) and tosic acid ( $15 \mathrm{mg}, 80 \mu \mathrm{mol}, 0.20$ equiv). The reaction was quenched after $18 \mathrm{~h}$. Purification by flash chromatography (PET/AcOEt, 4:1) afforded 7a (72 mg, 0.28 mmol, $70 \%$ ) as yellow oil. 
$R_{f} 0.35$ (PET/AcOEt 1:1, Anisaldehyde).

${ }^{1} \mathrm{H} \mathrm{NMR}\left(\mathrm{CDCl}_{3}, 400 \mathrm{MHz}\right) \delta 7.12(\mathrm{~d}, J=8.6 \mathrm{~Hz}, 2 \mathrm{H} ; \mathrm{ArH}), 6.88(\mathrm{~d}, J=8.6 \mathrm{~Hz}, 2 \mathrm{H} ; \mathrm{ArH})$, 4.20 - 3.99 (m, 2H; $\mathrm{CH}_{2} \mathrm{O}$ ), 3.81 (s, 3H; $\left.\mathrm{OCH}_{3}\right), 3.58$ (t, J = 5.2 Hz, 1H; CHPh), 2.59 - 2.47 (m, $\left.1 \mathrm{H} ; \mathrm{CH}_{2}\right), 2.45-2.27$ (m, 2H; $\left.\mathrm{CH}_{2}\right), 2.03-1.90$ (m, 3H; $\mathrm{CH}_{2}$ ), 1.85 (d, J = 6.0 Hz, 2H; $\mathrm{CH}_{2}$ ).

${ }^{1} \mathrm{H}$ NMR (benzene-d $\left.{ }^{6}, 400 \mathrm{MHz}\right) \delta$ 6.83-6.77 (m, 2H; ArH), 6.76-6.70 (m, 2H; ArH), 3.75-3.57 (m, 2H; $\mathrm{CH}_{2} \mathrm{O}$ ), 3.33 (s, 3H; $\mathrm{OCH}_{3}$ ), 3.02 (dd, $J=6.0,5.3 \mathrm{~Hz}, 1 \mathrm{H} ; \mathrm{CHAr}$ ), 2.37 (ddd, $J=16.6$, 9.6, 4.5 Hz, 1H; $\mathrm{CH}_{2} \mathrm{CO}$ ), 2.14 (ddd, $J=16.6,8.0,4.5 \mathrm{~Hz}, 1 \mathrm{H} ; \mathrm{CH}_{2} \mathrm{CO}$ ), 1.85 (tdd, $J=13.3,9.6$, 4.6 Hz, $\left.1 \mathrm{H} ; \mathrm{CH}_{2}\right), 1.62-1.49\left(\mathrm{~m}, 2 \mathrm{H} ; \mathrm{CH}_{2}\right), 1.45-1.35\left(\mathrm{~m}, 1 \mathrm{H} ; \mathrm{CH}_{2}\right), 1.32-1.20\left(\mathrm{~m}, 2 \mathrm{H} ; \mathrm{CH}_{2}\right)$.

${ }^{13} \mathrm{C} \mathrm{NMR}\left(\mathrm{CDCl}_{3}, 100 \mathrm{MHz}\right) \delta 193.2,158.5,146.8,133.3,132.1,128.9,114.1,65.8,55.2,45.0$, $34.9,30.9,25.1,21.8$.

IR $v\left(\mathrm{~cm}^{-1}\right)$ : $2934(\mathrm{~m}), 2870(\mathrm{w}), 1683$ (s), 1612 (w), 1511 (s), 1463 (w), 1385 (w), 1293 (w), 1247 (s), $1180(\mathrm{~m}), 1154(\mathrm{~m}), 1085(\mathrm{w}), 1035$ (m), $986(\mathrm{w}), 926(\mathrm{w}), 833(\mathrm{~m})$.

HRMS(ESI) calcd for $\mathrm{C}_{16} \mathrm{H}_{18} \mathrm{O}_{3}{ }^{+}(\mathrm{M}+\mathrm{H}) 259.1329$, found 259.1323 .

\section{Methyl 8-oxo-5-phenyl-3,4,5,6,7,8-hexahydro-2H-chromene-7-carboxylate (7c)}

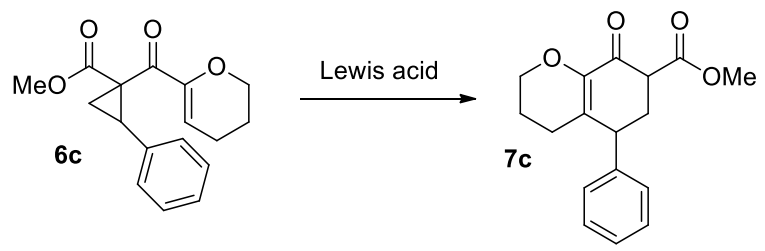

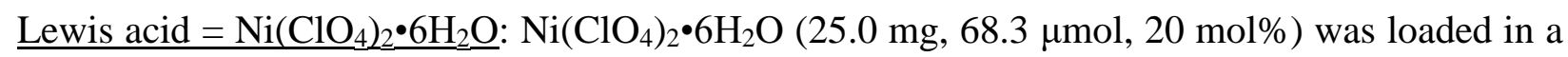
flask. Cyclopropane 6c (98 mg, 0.34 mmol, 1.0 equiv) diluted in DCM (3.4 mL) was added and the heterogeneous mixture was stirred at $23{ }^{\circ} \mathrm{C}$ for $26 \mathrm{~h}$. Then the reaction mixture was quenched with sat. $\mathrm{NaHCO}_{3}$ and diluted with DCM. The aqueous layer was extracted with DCM (x3). The organic layers were combined, dried over $\mathrm{MgSO}_{4}$ and evaporated under reduced pressure. The crude product was purified by column chromatography $\left(\mathrm{PET}_{\mathrm{Et}} \mathrm{O}\right.$ 1:1 then PET/AcOEt 2:1) to afford the homo-Nazarov product 7c $(62 \mathrm{mg}, 0.22 \mathrm{mmol}, 63 \%)$ as a mixture of anti/syn diastereoisomers and enol isomer.

$\underline{\text { Lewis acid }=\mathrm{BF}_{3}} \underline{\mathrm{Et}_{2}} \underline{\mathrm{O}}$ : Cyclopropane $\mathbf{6 c}(115 \mathrm{mg}, 402 \mu \mathrm{mol}, 1.00$ equiv) was dissolved in DCM $(3.8 \mathrm{~mL})$. A solution of the catalyst was prepared by diluting $500 \mu \mathrm{L}$ of $\mathrm{BF}_{3} \cdot \mathrm{Et}_{2} \mathrm{O}$ in 25 $\mathrm{mL}$ of DCM $(16 \mu \mathrm{mol} / 100 \mu \mathrm{L})$ and $250 \mu \mathrm{L}$ of this solution $(40.0 \mu \mathrm{mol}, 10 \mathrm{~mol} \%)$ were added to 
the reaction. The clear solution was stirred at $23^{\circ} \mathrm{C}$ for $2 \mathrm{~h} 45$. Then the reaction was quenched by adding sat. $\mathrm{NaHCO}_{3}$ and diluted with DCM. The aqueous layer was extracted with DCM (x3). The organic layers were combined, dried over $\mathrm{MgSO}_{4}$ and evaporated under reduced pressure. The crude product was purified by column chromatography (PET/Et $2 \mathrm{O} 1: 1$ then PET/AcOEt 2:1) to afford the homo-Nazarov product $7 \mathrm{c}(96 \mathrm{mg}, 0.34 \mathrm{mmol}, 83 \%)$ as a mixture of anti/syn diastereoisomers and enol isomer.

$\mathrm{R}_{f} 0.12(\mathrm{PET} / \mathrm{AcOEt} 3: 1)$.

${ }^{1} \mathrm{H} \mathrm{NMR}\left(\mathrm{CDCl}_{3}, 400 \mathrm{MHz}\right) \delta 12.35$ (s, 0.02H; OH), 7.39-7.32 (m, 2H; ArH), 7.31-7.27 (m, 1H; $\mathrm{ArH})$, 7.24-7.17 (m, 2H; ArH), 4.27 (m, 0.4H; $\left.\mathrm{CH}_{2} \mathrm{O}\right), 4.17-4.07$ (m, 1H; $\left.\mathrm{CH}_{2} \mathrm{O}\right), 3.85$ (m, 0.6H; $\mathrm{CH}_{2} \mathrm{O}$ ), 3.76 (m, 0.6H; CHCO), 3.72 (s, 1.6H; $\left.\mathrm{OCH}_{3}\right), 3.70\left(\mathrm{~s}, 1.1 \mathrm{H} ; \mathrm{OCH}_{3}\right), 3.68(\mathrm{~s}, 0.3 \mathrm{H}$; $\left.\mathrm{OCH}_{3}\right) 3.62(\mathrm{dd}, J=10.5,4.9 \mathrm{~Hz}, 0.4 \mathrm{H} ; \mathrm{CHCO}), 3.56(\mathrm{dd}, J=12.8,5.0 \mathrm{~Hz}, 0.4 \mathrm{H} ; \mathrm{CHPh}), 3.50$ (dd, $J=10.2,4.7 \mathrm{~Hz}, 0.6 \mathrm{H} ; \mathrm{CHPh}), 2.75$ (m, 0.6H; $\left.\mathrm{CH}_{2}\right), 2.54-2.33$ (m, 0.8H; $\left.\mathrm{CH}_{2}\right), 2.16$ (dt, $J=$ 13.5, 5.0 Hz, 0.6H; $\left.\mathrm{CH}_{2}\right), 2.08-1.73\left(\mathrm{~m}, 4 \mathrm{H} ; \mathrm{CH}_{2}\right)$.

Methyl 8-oxo-5-p-tolyl-3,4,5,6,7,8-hexahydro-2H-chromene-7-carboxylate (7d)

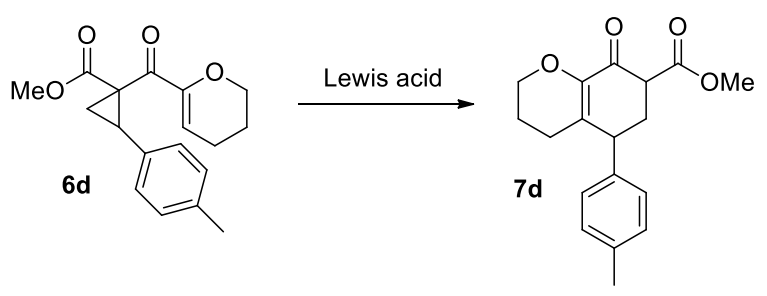

Lewis acid $=\mathrm{Ni}\left(\mathrm{ClO}_{4}\right)_{2} \cdot 6 \mathrm{H}_{2} \underline{\mathrm{O}}: \mathrm{Ni}\left(\mathrm{ClO}_{4}\right)_{2} \bullet 6 \mathrm{H}_{2} \mathrm{O}(27.9 \mathrm{mg}, 76.2 \mu \mathrm{mol}, 20 \mathrm{~mol} \%)$ was loaded in a flask. Cyclopropane $\mathbf{6 d}$ (115 mg, $383 \mu \mathrm{mol}, 1.00$ equiv) diluted in DCM (3.8 mL) was added and the heterogeneous mixture was stirred at $23{ }^{\circ} \mathrm{C}$ for $26 \mathrm{~h}$. Then the reaction mixture was quenched with sat. $\mathrm{NaHCO}_{3}$ and diluted with DCM. The aqueous layer was extracted with DCM (x3). The organic layers were combined, dried over $\mathrm{MgSO}_{4}$ and evaporated under reduced pressure. The crude product was purified by column chromatography $\left(\mathrm{PET}_{\mathrm{Et}} \mathrm{O}\right.$ 1:1 then PET/AcOEt 2:1) to afford the homo-Nazarov product $7 \mathbf{d}(97 \mathrm{mg}, 0.32 \mathrm{mmol}, 84 \%)$ as a mixture of anti/syn diastereoisomers and enol isomer.

Lewis acid $=\mathrm{BF}_{3} \cdot \mathrm{Et}_{2} \mathrm{O}$ : Cyclopropane $6 \mathbf{d}(121 \mathrm{mg}, 403 \mu \mathrm{mol}, 1.00$ equiv) was dissolved in DCM $(3.8 \mathrm{~mL})$. A solution of the catalyst was prepared by diluting $500 \mu \mathrm{L}$ of $\mathrm{BF}_{3} \bullet \mathrm{Et}_{2} \mathrm{O}$ in $25 \mathrm{ml}$ of DCM $(16 \mu \mathrm{mol} / 100 \mu \mathrm{L})$ and $250 \mu \mathrm{L}$ of this solution $(40.0 \mu \mathrm{mol}, 10 \mathrm{~mol} \%)$ were added to the reaction. The clear solution was stirred at $23^{\circ} \mathrm{C}$ for $2 \mathrm{~h} 45$. Then the reaction was quenched by 
adding sat. $\mathrm{NaHCO}_{3}$ and diluted with DCM. The aqueous layer was extracted with DCM (x3). The organic layers were combined, dried over $\mathrm{MgSO}_{4}$ and evaporated under reduced pressure. The crude product was purified by column chromatography (PET/Et $2 \mathrm{O} 1: 1$ then PET/AcOEt 2:1) to afford the homo-Nazarov product $7 \mathbf{d}(109 \mathrm{mg}, 363 \mu \mathrm{mol}, 90 \%)$ as a mixture of anti/syn diastereoisomers and enol isomer.

$\mathrm{R}_{f} 0.12$ (PET/AcOEt 3:1).

${ }^{1} \mathrm{H}$ NMR $\left(\mathrm{CDCl}_{3}, 400 \mathrm{MHz}\right) \delta$ 7.19-7.13 (m, 2H; ArH), 7.13-7.04 (m, 2H; ArH), 4.26 (m, 0.4H; $\mathrm{CH}_{2} \mathrm{O}$ ), 4.15-4.05 (m, $1 \mathrm{H} ; \mathrm{CH}_{2} \mathrm{O}$ ), 3.83 (m, 0.6H; $\mathrm{CH}_{2} \mathrm{O}$ ), 3.75-3.69 (m, 0.6H; CHCO), 3.72 (s, $\left.1.8 \mathrm{H} ; \mathrm{OCH}_{3}\right), 3.70\left(\mathrm{~s}, 1.1 \mathrm{H} ; \mathrm{OCH}_{3}\right), 3.68\left(\mathrm{~s}, 0.1 \mathrm{H} ; \mathrm{OCH}_{3}\right), 3.62-3.46(\mathrm{~m}, 1.4 \mathrm{H} ; \mathrm{CHCO}$ and $\mathrm{CHPh}), 2.72\left(\mathrm{~m}, 0.6 \mathrm{H} ; \mathrm{CH}_{2}\right), 2.50-2.31\left(\mathrm{~m}, 0.8 \mathrm{H} ; \mathrm{CH}_{2}\right), 2.34\left(\mathrm{~s}, 3 \mathrm{H} ; \mathrm{CH}_{3}\right), 2.13(\mathrm{dt}, J=13.4,4.9$ $\left.\mathrm{Hz}, 0.6 \mathrm{H} ; \mathrm{CH}_{2}\right), 2.05-1.73\left(\mathrm{~m}, 4 \mathrm{H} ; \mathrm{CH}_{2}\right)$.

\section{Methyl 5-(4-chlorophenyl)-8-oxo-3,4,5,6,7,8-hexahydro-2H-chromene-7-carboxylate (7e)}

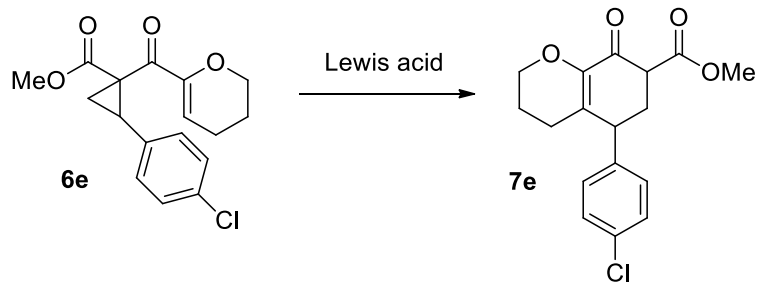

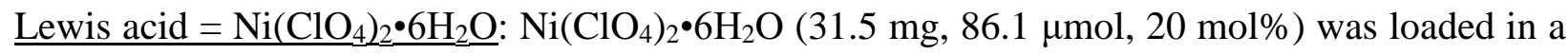
flask. Cyclopropane 6e (138 mg, $430 \mu \mathrm{mol}, 1.00$ equiv) diluted in DCM (4.3 mL) was added and the heterogeneous mixture was stirred at $23{ }^{\circ} \mathrm{C}$ for $26 \mathrm{~h}$. Then the reaction mixture was quenched with sat. $\mathrm{NaHCO}_{3}$ and diluted with DCM. The aqueous layer was extracted with DCM (x3). The organic layers were combined, dried over $\mathrm{MgSO}_{4}$ and evaporated under reduced pressure. The crude was purified by column chromatography (PET/Et $2 \mathrm{O}$ 1:1 then PET/AcOEt 2:1) to afford the homo-Nazarov product 7 e $(90 \mathrm{mg}, 0.28 \mathrm{mmol}, 65 \%)$ as a mixture of anti/syn diastereoisomers and enol isomer.

$\underline{\text { Lewis acid }=\mathrm{BF}_{3}} \cdot \mathrm{Et}_{2} \underline{\mathrm{O}}$ : Cyclopropane $6 \mathrm{e}(140 \mathrm{mg}, 436 \mu \mathrm{mol}, 1.00$ equiv $)$ was dissolved in $\operatorname{DCM}(4.1 \mathrm{~mL})$. A solution of the catalyst was prepared by diluting $500 \mu \mathrm{L}$ of $\mathrm{BF}_{3} \cdot \mathrm{Et}_{2} \mathrm{O}$ in 25 $\mathrm{mL}$ of DCM $(16 \mu \mathrm{mol} / 100 \mu \mathrm{L})$ and $280 \mu \mathrm{L}$ of this solution $(44.8 \mu \mathrm{mol}, 10 \mathrm{~mol} \%)$ were added to the reaction. The clear solution was stirred at $23^{\circ} \mathrm{C}$ for $2 \mathrm{~h} 45$. Then the reaction was quenched by adding sat. $\mathrm{NaHCO}_{3}$ and diluted with DCM. The aqueous layer was extracted with DCM (x3). The organic layers were combined, dried over $\mathrm{MgSO}_{4}$ and evaporated under reduced pressure. 
The crude product was purified by column chromatography (PET/Et $\mathrm{Ot}_{2} \mathrm{O}$ 1:1 then PET/AcOEt 2:1) to afford the homo-Nazarov product $7 \mathrm{e}(119 \mathrm{mg}, 371 \mu \mathrm{mol}, 85 \%)$ as a mixture of anti/syn diastereoisomers and enol isomer.

$\mathrm{R}_{f} 0.12$ (PET/AcOEt 3:1).

${ }^{1} \mathrm{H}$ NMR $\left(\mathrm{CDCl}_{3}, 400 \mathrm{MHz}\right) \delta$ 7.36-7.28 (m, 2H; ArH), 7.19-7.08 (m, 2H; ArH), 4.27 (m, 0.4H; $\mathrm{CH}_{2} \mathrm{O}$ ), 4.14-4.07 (m, 1H; $\mathrm{CH}_{2} \mathrm{O}$ ), 3.84 (m, 0.6H; $\mathrm{CH}_{2} \mathrm{O}$ ), 3.77-3.58 (m, 0.6H; CHCO), 3.73 (s, 1.7H; $\left.\mathrm{OCH}_{3}\right), 3.70\left(\mathrm{~s}, 1.1 \mathrm{H} ; \mathrm{OCH}_{3}\right), 3.69\left(\mathrm{~s}, 0.2 \mathrm{H} ; \mathrm{OCH}_{3}\right), 3.62(\mathrm{dd}, J=10.0,5.2 \mathrm{~Hz}, 0.4 \mathrm{H}$; CHCO), 3.55 (dd, $J=12.2,5.5 \mathrm{~Hz}, 0.4 \mathrm{H} ; \mathrm{CHPh}), 3.46$ (dd, $J=9.9,4.7 \mathrm{~Hz}, 0.6 \mathrm{H} ; \mathrm{CHPh}), 2.73$ (m, 0.6H; $\left.\mathrm{CH}_{2}\right), 2.48-2.30\left(\mathrm{~m}, 0.8 \mathrm{H} ; \mathrm{CH}_{2}\right), 2.11$ (dt, $\left.J=13.5,5.0 \mathrm{~Hz}, 0.6 \mathrm{H} ; \mathrm{CH}_{2}\right), 2.07-1.76(\mathrm{~m}$, $\left.4 \mathrm{H} ; \mathrm{CH}_{2}\right)$.

\section{5-Phenyl-3,4,6,7-tetrahydro-2H-chromen-8(5H)-one (7f)}

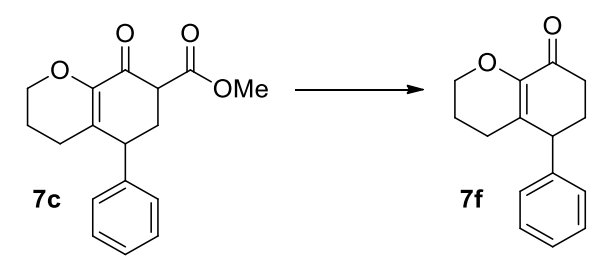

Ketoester 7c (62 mg, $0.22 \mathrm{mmol}, 1.0$ equiv) was dissolved in DMSO (1 mL) and water (0.1 mL). $\mathrm{NaCl}$ (30 mg, $0.51 \mathrm{mmol}, 2.4$ equiv) was added and the mixture was heated on microwave at $150^{\circ} \mathrm{C}$ for $1 \mathrm{~h}$. The reaction mixture was diluted with AcOEt and the organic layer was washed with brine (x5). The organic layer was dried over $\mathrm{MgSO}_{4}$ and evaporated under reduced pressure. The crude product was purified by column chromatography (PET/AcOEt 2:1) to afford the decarboxylated product $7 \mathbf{f}$ (49 $\mathrm{mg}, 0.22 \mathrm{mmol}$, quantitative).

$\mathrm{R}_{f} 0.50$ (PET/AcOEt 1:1).

${ }^{1} \mathrm{H}$ NMR $\left(\mathrm{CDCl}_{3}, 400 \mathrm{MHz}\right) \delta$ 7.42-7.33 (m, 2H; ArH), 7.33-7.27 (m, 1H; ArH), 7.26-7.20 (m, 2H; ArH), 4.12 (m, 2H; $\mathrm{OCH}_{2}$ ), 3.65 (t, $\left.J=5.3 \mathrm{~Hz}, 1 \mathrm{H} ; \mathrm{CHPh}\right), 2.55$ (m, 1H; $\mathrm{CH}_{2}$ ), 2.48-2.35 (m, 2H; $\mathrm{CH}_{2}$ ), 2.07-1.97 (m, 3H; $\left.\mathrm{CH}_{2}\right), 1.92-1.82\left(\mathrm{~m}, 2 \mathrm{H} ; \mathrm{CH}_{2}\right)$.

${ }^{13} \mathrm{C} \mathrm{NMR}\left(\mathrm{CDCl}_{3} 100 \mathrm{MHz}\right) \delta 193.2,147.1,141.4,131.7,128.8,128.1,127.05,66.0,45.9,35.0$, $30.9,25.2,21.9$.

IR v (cm-1): $3347(\mathrm{w}), 2945(\mathrm{w}), 2864(\mathrm{w}), 2146(\mathrm{w}), 1682(\mathrm{~s}), 1631(\mathrm{w}), 1491(\mathrm{w}), 1453(\mathrm{w})$, $1386(\mathrm{w}), 1293$ (m), 1154 (s), $1086(\mathrm{w}), 985$ (w), $912(\mathrm{w}), 738(\mathrm{~m}), 705$ (m). 
HRMS (ESI) calcd for $\mathrm{C}_{15} \mathrm{H}_{17} \mathrm{O}_{2}{ }^{+}(\mathrm{M}+\mathrm{H})$ 229.1228; found 229.1231.

\section{5-p-Tolyl-3,4,6,7-tetrahydro-2H-chromen-8(5H)-one (7g)}

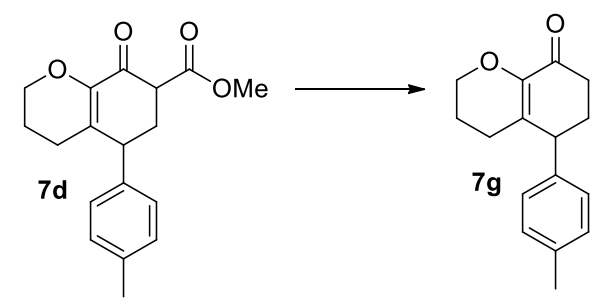

Ketoester 7d (100 mg, $333 \mu \mathrm{mol}, 1.00$ equiv) was dissolved in DMSO (1 mL) and water (0.1 $\mathrm{mL}$ ). $\mathrm{NaCl}$ (50 mg, $0.90 \mathrm{mmol}, 2.6$ equiv) was added and the mixture was heated on microwave at $150^{\circ} \mathrm{C}$ for $1 \mathrm{~h} 40$. The reaction mixture was diluted with AcOEt and the organic layer was washed with brine (x5). The organic layer was dried over $\mathrm{MgSO}_{4}$ and evaporated under reduced pressure. The crude product was purified by column chromatography (PET/AcOEt 2:1) to afford the decarboxylated product $\mathbf{7 g}$ (62 $\mathrm{mg}, 0.26 \mathrm{mmol}, 77 \%)$.

$\mathrm{R}_{f} 0.53$ (PET/AcOEt 1:1).

${ }^{1} \mathrm{H} \mathrm{NMR}\left(\mathrm{CDCl}_{3}, 400 \mathrm{MHz}\right) \delta 7.18(\mathrm{~d}, J=7.7 \mathrm{~Hz}, 2 \mathrm{H} ; \mathrm{ArH}), 7.12(\mathrm{~d}, J=8.1 \mathrm{~Hz}, 2 \mathrm{H} ; \mathrm{ArH}), 4.12$ (m, 2H; $\left.\mathrm{OCH}_{2}\right), 3.61$ (t, J = 5.5 Hz, 1H; CHPh), 2.55 (m, 1H; $\left.\mathrm{CH}_{2}\right), 2.47-2.31\left(\mathrm{~m}, 2 \mathrm{H} ; \mathrm{CH}_{2}\right)$, 2.37 (s, 3H; $\left.\mathrm{CH}_{3}\right), 2.05-1.94\left(\mathrm{~m}, 3 \mathrm{H} ; \mathrm{CH}_{2}\right), 1.91-1.81\left(\mathrm{~m}, 2 \mathrm{H} ; \mathrm{CH}_{2}\right)$.

${ }^{13} \mathrm{C} \mathrm{NMR}\left(\mathrm{CDCl}_{3} 100 \mathrm{MHz}\right) \delta 193.3,147.0,138.4,136.7,132.0,129.5,128.0,66.0,45.5,35.0$, $30.9,25.2,21.9,21.1$.

IR v (cm-1): 2933 (w), 2867 (w), $2246(w), 1683$ (s), 1628 (w), $1513(w), 1450(w), 1384(w)$, 1292 (m), 1184 (w), 1151 (s), 1085 (w), 986 (w), 913 (w), 819 (w), 732 (m).

HRMS (ESI) calcd for $\mathrm{C}_{16} \mathrm{H}_{19} \mathrm{O}_{2}{ }^{+}(\mathrm{M}+\mathrm{H})$ 243.1385; found 243.1397.

\section{5-(4-Chlorophenyl)-3,4,6,7-tetrahydro-2H-chromen-8(5H)-one (7h)}

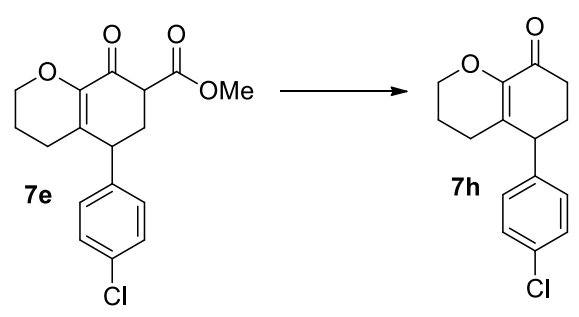


Ketoester 7e (90 mg, $0.28 \mathrm{mmol}, 1.0$ equiv) was dissolved in DMSO (2 mL) and water (0.2 mL). $\mathrm{NaCl}$ (120 mg, $2.05 \mathrm{mmol}, 7.10$ equiv) was added and the mixture was heated on microwave at $150^{\circ} \mathrm{C}$ for $1 \mathrm{~h} 30$. The reaction mixture was diluted with AcOEt and the organic layer was washed with brine (x5). The organic layer was dried over $\mathrm{MgSO}_{4}$ and evaporated under reduced pressure. The crude was purified by column chromatography (PET/AcOEt 2:1) to afford the decarboxylated product $7 \mathbf{h}$ (42 $\mathrm{mg}, 0.16 \mathrm{mmol}, 57 \%)$.

$\mathrm{R}_{f} 0.45$ (PET/AcOEt 1:1).

${ }^{1} \mathrm{H} \mathrm{NMR}\left(\mathrm{CDCl}_{3}, 400 \mathrm{MHz}\right) \delta 7.34(\mathrm{~d}, J=8.4 \mathrm{~Hz}, 2 \mathrm{H} ; \mathrm{ArH}), 7.17(\mathrm{~d}, J=8.4 \mathrm{~Hz}, 2 \mathrm{H} ; \mathrm{ArH}), 4.12$ (m, 2H; $\left.\mathrm{OCH}_{2}\right), 3.63$ (t, $\left.J=5.5 \mathrm{~Hz}, 1 \mathrm{H} ; \mathrm{CHPh}\right), 2.59-2.30\left(\mathrm{~m}, 3 \mathrm{H} ; \mathrm{CH}_{2}\right), 2.05-1.92(\mathrm{~m}, 3 \mathrm{H}$; $\left.\mathrm{CH}_{2}\right), 1.92-1.83\left(\mathrm{~m}, 2 \mathrm{H} ; \mathrm{CH}_{2}\right)$.

${ }^{13} \mathrm{C} \mathrm{NMR}\left(\mathrm{CDCl}_{3} 100 \mathrm{MHz}\right) \delta 192.9,147.19,140.0,132.9,130.9,129.4,129.0,66.0,45.3,34.9$, $30.8,25.2,21.8$.

IR $v\left(\mathrm{~cm}^{-1}\right)$ : $2934(\mathrm{w}), 2872(\mathrm{w}), 2249$ (w), $1683(\mathrm{~s}), 1631(\mathrm{w}), 1489(\mathrm{w}), 1385(\mathrm{w}), 1293(\mathrm{w})$, $1155(\mathrm{~m}), 1088(\mathrm{w}), 1015(\mathrm{w}), 913(\mathrm{w}), 832(\mathrm{w}), 735(\mathrm{~m})$.

HRMS (ESI) calcd for $\mathrm{C}_{15} \mathrm{H}_{16} \mathrm{ClO}_{2}^{+}(\mathrm{M}+\mathrm{H})$ 263.0839; found 263.0844.

\section{Asymmetric induction}

1-tert-Butyl 3-methyl 2-diazomalonate (81)

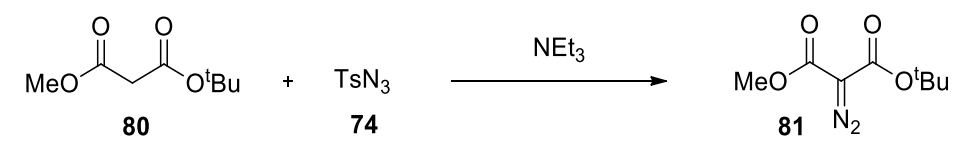

Following the same procedure described precedently, ${ }^{[6]}$ tert-butyl methyl malonate diester $(\mathbf{8 0})$ ( $0.49 \mathrm{~mL}, 2.9 \mathrm{mmol}, 1.0$ equiv), triethylamine $(0.44 \mathrm{~mL}, 3.2 \mathrm{mmol}, 1.1$ equiv) and tosyl azide (74) (630 mg, $3.16 \mathrm{mmol}, 1.10$ equiv) afforded tert-butyl-3-methyl-2-diazomalonate (81) (460 $\mathrm{mg}, 2.32 \mathrm{mmol}, 81 \%)$.

$\mathrm{R}_{f} 0.59\left(\mathrm{PET}_{\mathrm{Et}} \mathrm{O}\right.$ 1:1).

${ }^{1} \mathrm{H}$ NMR $\left(\mathrm{CDCl}_{3}, 400 \mathrm{MHz}\right) \delta 3.85\left(\mathrm{~s}, 1 \mathrm{H} ; \mathrm{OCH}_{3}\right), 1.54\left(\mathrm{~s}, 9 \mathrm{H} ; \mathrm{CH}_{3}\right)$. 


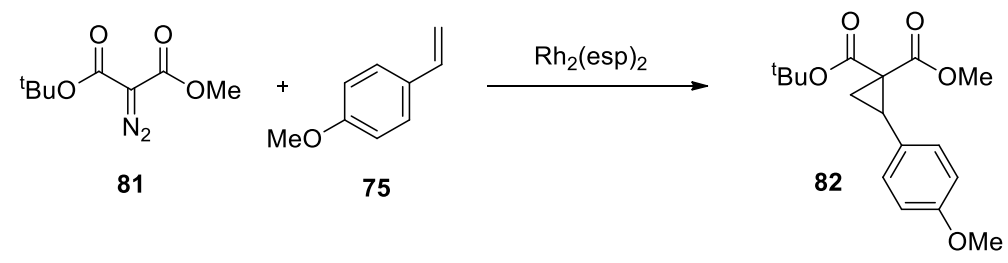

The reaction was performed following the GP1, using $\mathrm{Rh}_{2}(\mathrm{esp})_{2}(0.9 \mathrm{mg}, 1 \mu \mathrm{mol}, 0.1 \mathrm{~mol} \%)$, 4 methoxystyrene (75) $(0.16 \mathrm{~mL}, 1.2 \mathrm{mmol}, 1.00$ equiv) and diazomalonate 81 (200 mg, 1.00 mmol, 1.00 equiv). Purification by column chromatography ( $\mathrm{PET}_{\mathrm{Et}} \mathrm{O}$ 10:1 to 5:1) afforded cyclopropane 82 (276 mg, $0.90 \mathrm{mmol}, 90 \%$ ).

$\mathrm{R}_{f} 0.45\left(\mathrm{PET}_{\mathrm{Et}} \mathrm{O}\right.$ 5:1).

${ }^{1} \mathrm{H} \mathrm{NMR}\left(\mathrm{CDCl}_{3}, 400 \mathrm{MHz}\right) \delta 7.14(\mathrm{~d}, J=8.8 \mathrm{~Hz}, 2 \mathrm{H} ; \mathrm{ArH}), 6.82(\mathrm{~d}, J=8.8 \mathrm{~Hz}, 2 \mathrm{H} ; \mathrm{ArH}), 3.80$ (s, 3H; $\left.\mathrm{OCH}_{3}\right), 3.43$ (s, 3H; $\left.\mathrm{OCH}_{3}\right), 3.10(\mathrm{t}, J=8.2 \mathrm{~Hz}, 1 \mathrm{H} ; \mathrm{CHPh}), 2.07$ (dd, $J=7.7,5.0 \mathrm{~Hz}$, $\left.1 \mathrm{H} ; \mathrm{CH}_{2}\right), 1.65$ (dd, $\left.J=9.3,5.2 \mathrm{~Hz}, 1 \mathrm{H} ; \mathrm{CH}_{2}\right), 1.50\left(\mathrm{~s}, 9 \mathrm{H} ; \mathrm{CH}_{3}\right)$.

tButyl-1-(3,4-dihydro-2H-pyran-6-carbonyl)-2-(4-methoxyphenyl)cyclopropanecarboxylate (6g) (trans/cis)

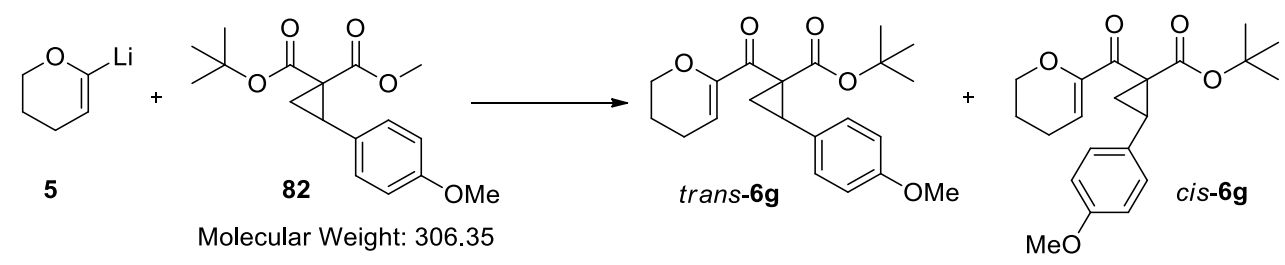

Following a reported procedure, ${ }^{[11]}$ dihydropyran (44) $(85 \mu \mathrm{L}, 0.93 \mathrm{mmol}, 1.0$ equiv) was dissolved in THF $(0.13 \mathrm{~mL})$ and the mixture was cooled to $-78^{\circ} \mathrm{C}$. $t \mathrm{BuLi}(1.6 \mathrm{M}$ solution in pentane, $0.56 \mathrm{~mL}, 0.90 \mathrm{mmol}, 1.0$ equiv) was added dropwise to this solution at the same temperature. The reaction mixture was stirred at $0^{\circ} \mathrm{C}$ for $30 \mathrm{~min}$. Then THF $(3.0 \mathrm{~mL})$ was added and the solution was cooled to $-95^{\circ} \mathrm{C}$. The 1,1-cyclopropane diester 82 ( $270 \mathrm{mg}, 0.90 \mathrm{mmol}, 1.00$ equiv) dissolved in THF ( $1 \mathrm{~mL})$ was added in one portion to this solution. The reaction mixture was allowed until the temperature of the cooling bath was $-40^{\circ} \mathrm{C}(1.30 \mathrm{~h})$. Then the reaction mixture was poured in brine and diluted with DCM. The aqueous layer was extracted with DCM (x3). The organic layers were combined, dried over $\mathrm{Na}_{2} \mathrm{SO}_{4}$ and evaporated under reduced pressure. A 3/1 mixture of trans/cis is detected by ${ }^{1} \mathrm{H}$ NMR of the crude. Purification by column

[11] Boeckman, R. K.; Bruza, K. J. Tetrahedron 1981, 37, 3997. 
chromatography (PET/Et $2 \mathrm{O} 4: 1)$ afforded the trans isomer of $\mathbf{6 g}(111 \mathrm{mg}, 0.31 \mathrm{mmol}, 34 \%)$, a mixture of cis/trans isomers of $\mathbf{6 g}(34 \mathrm{mg}, 11 \mu \mathrm{mol}, 12 \%)$ and starting material $82(71 \mathrm{mg}, 0.23$ mmol, $25 \%$ ) as oils.

Trans isomer

$\mathrm{R}_{f} 0.41$ (PET/AcOEt 4:1).

${ }^{1} \mathrm{H} \mathrm{NMR}\left(\mathrm{CDCl}_{3}, 400 \mathrm{MHz}\right) \delta 7.03(\mathrm{~d}, J=8.6 \mathrm{~Hz}, 2 \mathrm{H} ; \mathrm{ArH}), 6.73(\mathrm{~d}, J=8.8 \mathrm{~Hz}, 2 \mathrm{H}$; ArH), 5.67 (t, $J=4.2 \mathrm{~Hz}, 1 \mathrm{H}$; alkeneH), $3.98\left(\mathrm{~m}, 1 \mathrm{H} ; \mathrm{OCH}_{2}\right), 3.88\left(\mathrm{~m}, 1 \mathrm{H} ; \mathrm{OCH}_{2}\right), 3.76\left(\mathrm{~s}, 3 \mathrm{H} ; \mathrm{OCH}_{3}\right), 3.21$ (t, $J=8.3 \mathrm{~Hz}, 1 \mathrm{H} ; \mathrm{CHPh}), 2.13\left(\mathrm{dd}, J=7.9,5.0 \mathrm{~Hz}, 1 \mathrm{H} ; \mathrm{CH}_{2}\right), 2.10-1.96\left(\mathrm{~m}, 2 \mathrm{H} ; \mathrm{CH}_{2}\right), 1.71$ (td, $\left.J=6.7,3.5 \mathrm{~Hz}, 2 \mathrm{H} ; \mathrm{CH}_{2}\right), 1.51-1.44\left(\mathrm{~m}, 1 \mathrm{H} ; \mathrm{CH}_{2}\right), 1.44\left(\mathrm{~s}, 9 \mathrm{H} ; \mathrm{CH}_{3}\right)$.

${ }^{13} \mathrm{C} \mathrm{NMR}\left(\mathrm{CDCl}_{3} 125 \mathrm{MHz}\right) \delta 189.4,169.8,158.4,150.8,129.5,126.3,113.3,109.2,101.1$, 100.0, 81.6, 66.0, 55.1, 41.3, 31.8, 27.9, 21.6, 20.5, 16.7.

IR $v\left(\mathrm{~cm}^{-1}\right): 2986(\mathrm{w}), 2953(\mathrm{w}), 2936$ (w), $1724(\mathrm{~m}), 1723(\mathrm{~m}), 1697(\mathrm{w}), 1632$ (m), $1614(\mathrm{w})$, 1517 (s), 1463 (w), 1460 (w), 1459 (w), 1442 (w), 1369 (w), 1339 (w), 1320 (m), 1290 (s), 1251 (s), $1213(\mathrm{w}), 1212$ (w), 1176 (m), 1150 (s), 1120 (w), 1062 (w), 1036 (w), 1020 (w), 920 (m), $914(\mathrm{~m}), 913(\mathrm{~m}), 838(\mathrm{~m}), 737(\mathrm{~s})$.

HRMS (ESI) calcd for $\mathrm{C}_{21} \mathrm{H}_{27} \mathrm{O}_{5}^{+}(\mathrm{M}+\mathrm{H}) 359.1853$; found 359.1843.

\section{5-(4-Methoxyphenyl)-3,4,6,7-tetrahydro-2H-chromen-8(5H)-one (7a)}

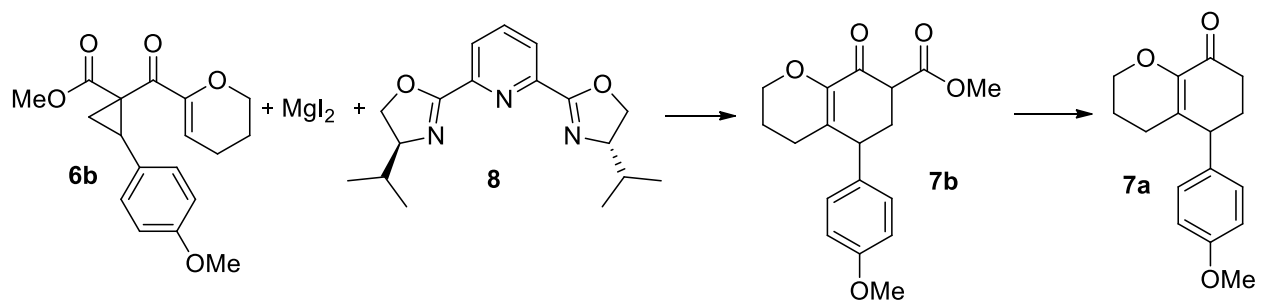

$\mathrm{MgI}_{2}(20.2 \mathrm{mg}, 72.6 \mu \mathrm{mol}, 0.28$ equiv) and PyBOX ligand 8 (24.1 mg, $80.0 \mu \mathrm{mol}, 0.30$ equiv) were stirred in DCM $(1 \mathrm{~mL})$ with dried MS $4 \AA$ for $45 \mathrm{~min}$ at $23^{\circ} \mathrm{C}$. Then, cyclopropane $6 \mathbf{b}(83$ $\mathrm{mg}, 0.26 \mathrm{mmol}, 1$ equiv) dissolved in DCM (1.6 mL) was added and the reaction mixture was stirred at $23^{\circ} \mathrm{C}$ for 4.5 days (complete conversion was achieved). The reaction mixture was filtered over a short column of silica gel (PET/Et ${ }_{2} \mathrm{O} 1 / 1$ then PET/AcOEt 1/1). The collected fractions were dissolved in AcOEt and the organic layer was washed with an aqueous solution of $\mathrm{Na}_{2} \mathrm{~S}_{2} \mathrm{O}_{3}$ (x2). The organic layer was dried over $\mathrm{MgSO}_{4}$ and concentrated under reduced 
pressure. Purification by column chromatography (PE/AcOEt $3 / 1$ to $2 / 1)$ afforded $\mathbf{7 b}$ (40 mg, $0.12 \mathrm{mmol}, 48 \%$ ) as a yellow oil.

Part of $7 \mathbf{b}(10 \mathrm{mg}, 31.6 \mu \mathrm{mol}, 1$ equiv) was dissolved in DMSO $(2 \mathrm{~mL})$ and water $(0.2 \mathrm{~mL})$. $\mathrm{NaCl}(100 \mathrm{mg}, 1.7 \mathrm{mmol})$ was added and the reaction mixture was heated in microwave at $150^{\circ} \mathrm{C}$ for $30 \mathrm{~min}$. The reaction was diluted with AcOEt and the organic layer was washed with brine (x5). The organic layer was dried over $\mathrm{MgSO}_{4}$ and concentrated under reduced pressure to give 7a (no complete conversion was achieved but the product is detected by ${ }^{1} \mathrm{H} N \mathrm{NM}$ ).

Optical purity determined by chiral HPLC analysis: $11 \%$.

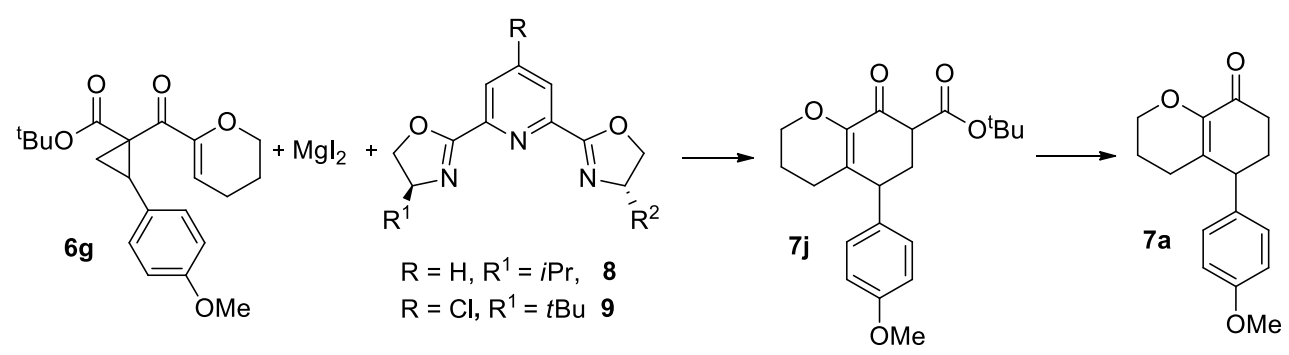

Following a reported procedure, ${ }^{[12]} \mathrm{MgI}_{2}(2.1 \mathrm{mg}, 7.6 \mu \mathrm{mol}, 0.14$ equiv) and PyBOX ligand 8 (2.6 mg, $8.6 \mu \mathrm{mol}, 0.15$ equiv) or 9 (3.1 mg, $8.6 \mu \mathrm{mol}, 0.15$ equiv) were stirred in DCM $(0.5 \mathrm{~mL})$ for $45 \mathrm{~min}$ at $23^{\circ} \mathrm{C}$. Then, cyclopropane $\mathbf{6 g}(20 \mathrm{mg}, 56 \mu \mathrm{mol}, 1$ equiv) dissolved in DCM (0.5 $\mathrm{mL}$ ) was added and the reaction mixture was stirred at $23^{\circ} \mathrm{C}$ for $22 \mathrm{~h}$ (no complete conversion was achieved). The reaction was quenched by adding sat. $\mathrm{NaHCO}_{3}$. The aqueous layer was extracted with AcOEt, dried over $\mathrm{MgSO}_{4}$ and evaporated under reduced pressure. The crude product was purified by column chromatography (PET/AcOEt 2/1) and the collected fractions were directly engaged in the decarboxylation step. Following a reported procedure, ${ }^{[13]} \mathbf{7} \mathbf{j}$ was dissolved in a mixture DCM/TFA $(250 \mu \mathrm{l} / 50 \mu \mathrm{l})$ and stirred at RT for $20 \mathrm{~h}$. The solution was diluted with DCM and saturated aqueous $\mathrm{NaHCO}_{3}$ was added. After gas evolution had ceased, the layers were separated and the aqueous layer was washed with additional DCM (x2). The combined organic layers were dried over $\mathrm{MgSO}_{4}$ and concentrated under reduced pressure to give $7 \mathbf{a}$.

Optical purity determined by chiral HPLC analysis : 25\% (with both ligands). 


\section{Amino- and oxycyclopropanes synthesis}

\section{3,4-Dihydro-2H-pyridine-1-carboxylic acid benzyl ester (26)}

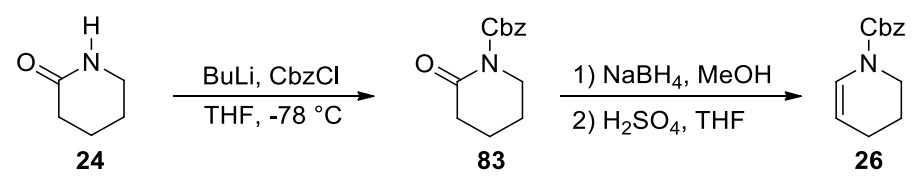

Following a reported procedure, ${ }^{[14]} \delta$-valerolactam 24 (3.00 g, $30.2 \mathrm{mmol}, 1.00$ equiv) was dissolved in THF $(130 \mathrm{~mL})$. The reaction mixture was cooled to $-78{ }^{\circ} \mathrm{C}$ and ${ }^{n} \mathrm{BuLi}(2.5 \mathrm{M}$ in hexane, $12 \mathrm{~mL}, 30 \mathrm{mmol}, 1.0$ equiv) was added dropwise to the resulting suspension. After 30 min at $-78{ }^{\circ} \mathrm{C}$, a solution of benzylchloroformate $(4.3 \mathrm{~mL}, 30 \mathrm{mmol}, 1.0$ equiv) in THF (30 mL) was added dropwise. After $4 \mathrm{~h}$ at $-78^{\circ} \mathrm{C}$, the reaction mixture was quenched with sat. $\mathrm{NH}_{4} \mathrm{Cl}(40$ $\mathrm{mL})$ and warmed to $23{ }^{\circ} \mathrm{C}$. The reaction mixture was extracted with $\mathrm{Et}_{2} \mathrm{O}(3 \times 50 \mathrm{~mL})$. The combined organic layers were washed with brine $(50 \mathrm{~mL})$, dried over $\mathrm{MgSO}_{4}$ and the solvent was removed under reduced pressure to give the crude $\mathrm{Cbz}$ protected lactam $\mathbf{8 3}$ (6.95 g, 29.8 mmol, 99\%), which was used without further purification.

$R_{f} 0.30$ (PET/AcOEt 2:1, Anisaldehyde);

${ }^{1} \mathrm{H}$ NMR $\left(\mathrm{CDCl}_{3}, 400 \mathrm{MHz}\right) \delta$ 7.47-7.28 (m, 5H; ArH), $5.28\left(\mathrm{~s}, 2 \mathrm{H} ; \mathrm{OCH}_{2}\right), 3.74(\mathrm{~m}, 2 \mathrm{H}$; $\left.\mathrm{CH}_{2} \mathrm{~N}\right), 2.54\left(\mathrm{~m}, 2 \mathrm{H} ; \mathrm{CH}_{2} \mathrm{CO}\right), 1.83\left(\mathrm{~m}, 4 \mathrm{H} ; \mathrm{CH}_{2}\right)$.

Following a reported procedure, ${ }^{[15]}$ protected lactam 83 (2.82 g, $12.1 \mathrm{mmol}, 1.00$ equiv) was dissolved in methanol $(52 \mathrm{~mL})$ at $0{ }^{\circ} \mathrm{C}$ and sodium borohydride $(0.46 \mathrm{~g}, 12 \mathrm{mmol}, 1.0$ equiv) was added portionswise. After the end of the addition, the reaction mixture was stirred at $0{ }^{\circ} \mathrm{C}$ for 15 min and poured onto ice-water $(150 \mathrm{~mL})$. The reaction mixture was extracted with AcOEt $(3 \times 100 \mathrm{~mL})$. The combined organic layers were washed with brine $(50 \mathrm{~mL})$, the combined water layers were extracted with AcOEt $(100 \mathrm{~mL})$ and the combined organic layers were dried over $\mathrm{MgSO}_{4}$ and the solvent was removed under reduced pressure.

The residues were dried in HV for $15 \mathrm{~min}$ and dissolved in THF (25 mL). Conc. sulfuric acid $(0.13 \mathrm{~mL})$ was added and the reaction was monitored via TLC (PET/AcOEt 10:1-2:1). After $1 \mathrm{~h}$ the reaction mixture was poured onto sat. $\mathrm{NaHCO}_{3}(100 \mathrm{~mL})$ and extracted with AcOEt $(3 \times 100$

[14] A. Giovannini, D. Savoia, A. Umani-Ronchi, J. Org. Chem. 1989, 54, 228.

[15] Y. Takeuchi, K. Azuma, M. Oshige, H. Abe, H. Nishioka, K. Sasaki, T. Harayama, Tetrahedron 2003, 59, 1639. 
$\mathrm{mL}$ ). The combined organic layers were dried over $\mathrm{MgSO}_{4}$ and the solvent was removed under reduced pressure. The crude product was purified by flash column chromatography (PET/AcOEt 10:1-2:1) to yield enamide $\mathbf{2 6}(1.76 \mathrm{~g}, 8.11 \mathrm{mmol}, 67 \%)$ as a colorless oil.

$R_{f} 0.80$ (PET/AcOEt 2:1, Anisaldehyde).

${ }^{1} \mathrm{H} \mathrm{NMR}\left(\mathrm{CDCl}_{3}, 400 \mathrm{MHz}\right) \delta$ 7.43-7.27 (m, 5H; ArH), 6.89 (d, $J=8.6 \mathrm{~Hz}, 0.4 \mathrm{H}$; alkeneH), 6.80 $\left(\mathrm{d}, J=8.6 \mathrm{~Hz}, 0.6 \mathrm{H}\right.$; alkeneH), $5.18\left(\mathrm{~s}, 2 \mathrm{H} ; \mathrm{CH}_{2} \mathrm{O}\right), 4.97(\mathrm{~m}, 0.4 \mathrm{H}$; alkeneH), $4.86(\mathrm{~m}, 0.6 \mathrm{H}$; alkeneH), $3.63\left(\mathrm{~m}, 2 \mathrm{H} ; \mathrm{CH}_{2} \mathrm{~N}\right), 2.04\left(\mathrm{~m}, 2 \mathrm{H} ; \mathrm{CH}_{2}\right), 1.83\left(\mathrm{~m}, 2 \mathrm{H} ; \mathrm{CH}_{2}\right)$.

${ }^{13} \mathrm{C} \mathrm{NMR}\left(\mathrm{CDCl}_{3}, 100 \mathrm{MHz}\right)$ (rotamers!) $\delta 151.7,136.3,128.5,128.5,128.2,128.0,128.0$, $125.3,124.8,106.7,106.4,67.4,67.3,42.3,42.2,21.6,21.4,21.2$.

IR v (cm-1): $2951(\mathrm{w}), 1703$ (s), 1653 (m), 1409 (s), 1347 (s), 1256 (s), 1227 (m), 1183 (w), 1108 (s), 1054 (m), $912(\mathrm{~m}), 764(\mathrm{~m}), 731$ (s), 697 (s).

${ }^{1} \mathrm{H}$ NMR corresponded to the literature values. ${ }^{[15]}$

2-Benzyl 7-ethyl 2-azabicyclo[4.1.0]heptane-2,7-dicarboxylate (27) and 2(benzyloxycarbonyl)-2-azabicyclo[4.1.0]heptane-7-carboxylic acid (28)

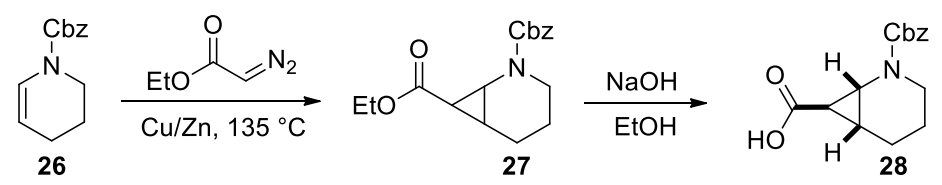

Following a reported procedure, ${ }^{[16]}$ a mixture of enamide 26 (1.70 g, $7.82 \mathrm{mmol}, 1.00$ equiv) and copper/bronze $(67 \mathrm{mg}$, prepared freshly as following: Zinc powder $(0.20 \mathrm{~g})$ was added to a solution of $\mathrm{CuSO}_{4} \cdot 5 \mathrm{H}_{2} \mathrm{O}(1.0 \mathrm{~g})$ in water $(2 \mathrm{~mL})$ over $10 \mathrm{~min}$ at RT. The resulting suspension was filtered, the solid was washed with water $(3 \times 5 \mathrm{~mL})$, ethanol $(3 \times 5 \mathrm{~mL})$ and $\mathrm{Et}_{2} \mathrm{O}(2 \times 5 \mathrm{~mL})$ and dried $1 \mathrm{~h}$ in $\mathrm{HV}$ ) was heated to $135^{\circ} \mathrm{C}$. Ethyl diazoacetate $(4.2 \mathrm{~mL}, 40 \mathrm{mmol}, 5.0$ equiv) was added via syringe pump over $90 \mathrm{~min}$, and the reaction was stirred for further $30 \mathrm{~min}$ before cooling down to RT. The crude product was purified by two successive flash column chromatography (PET/AcOEt 10:1-2:1) to yield cyclopropane $27\left(1.18 \mathrm{~g}, 3.89 \mathrm{mmol}, 50 \%, \mathrm{R}_{\mathrm{f}}=\right.$ 0.30 (PET/AcOEt 3:1), still containing some polymeric impurities) as a colorless oil.

[16] P. A. Grieco, M. D. Kaufman, J. Org. Chem. 1999, 64, 7586. 
The crude ester 27 (1.18 g, $3.89 \mathrm{mmol}, 1.00$ equiv) was dissolved in ethanol (4 mL) at $0{ }^{\circ} \mathrm{C}$ and $\mathrm{NaOH}\left(2.2 \mathrm{~g}, 55 \mathrm{mmol}, 14\right.$ equiv) was added. The reaction mixture was stirred $1 \mathrm{~h}$ at $0{ }^{\circ} \mathrm{C}$ and $12 \mathrm{~h}$ at $\mathrm{RT}$, diluted with water $(10 \mathrm{~mL})$, acidified to $\mathrm{pH}=1$ with $1 \mathrm{M} \mathrm{HCl}$ and extracted with $\mathrm{CH}_{2} \mathrm{Cl}_{2}(3 \times 20 \mathrm{~mL})$. The combined organic layers were dried over $\mathrm{MgSO}_{4}$ and the solvent was removed under reduced pressure to give nearly pure acid $\mathbf{2 8}(0.89 \mathrm{~g}, 3.2 \mathrm{mmol}, 84 \%, 42 \%$ from enamide 26) as a colorless oil.

${ }^{1} \mathrm{H} \mathrm{NMR}\left(\mathrm{CDCl}_{3}, 400 \mathrm{MHz}\right) \delta$ 7.39-7.26 (m, 5H; $\left.\mathrm{ArH}\right), 5.26-5.11\left(\mathrm{~m}, 2 \mathrm{H} ; \mathrm{OCH}_{2}\right), 3.88(\mathrm{dt}, J=$ $12.5 \mathrm{~Hz}, 3.2 \mathrm{~Hz}, 0.7 \mathrm{H} ; \mathrm{NCH}_{2}$ or $\mathrm{NCH}$ rotamer A), 3.74 (s, $0.3 \mathrm{H} ; \mathrm{NCH}_{2}$ or $\mathrm{NCH}$ rotamer $\left.\mathrm{B}\right), 3.57$ (m, $0.3 \mathrm{H} ; \mathrm{NCH}_{2}$ or $\mathrm{NCH}$ rotamer $\left.\mathrm{B}\right), 3.50\left(\mathrm{dd}, J=8.6 \mathrm{~Hz}, 2.6 \mathrm{~Hz}, 0.7 \mathrm{H} ; \mathrm{NCH}_{2}\right.$ or $\mathrm{NCH}$ rotamer A), $2.77\left(\mathrm{dd}, J=1.3 \mathrm{~Hz}, 0.6 \mathrm{~Hz}, 0.3 \mathrm{H} ; \mathrm{NCH}_{2}\right.$ or $\mathrm{NCH}$ rotamer B), $2.67(\mathrm{td}, J=12.5 \mathrm{~Hz}, 2.2 \mathrm{~Hz}$, $0.7 \mathrm{H} ; \mathrm{NCH}_{2}$ or $\mathrm{NCH}$ rotamer A), 2.08-1.78 (m, 3H; $\mathrm{CHCO}$ and $\left.\mathrm{CH}_{2}\right), 1.77-1.58(\mathrm{~m}, 2 \mathrm{H}$; $\mathrm{CH}$ or $\left.\mathrm{CH}_{2}\right), 1.40-1.20\left(\mathrm{~m}, 1 \mathrm{H} ; \mathrm{CH}\right.$ or $\left.\mathrm{CH}_{2}\right)$.

${ }^{13} \mathrm{C} \mathrm{NMR}\left(\mathrm{CDCl}_{3}, 100 \mathrm{MHz}\right)$ (rotamers!) $\delta$ 178.0, 177.0, 156.4, 136.5, 136.3, 128.8, 128.6, $128.5,128.3,127.7,127.6,127.5,127.2,126.7,126.4,68.4,67.0,39.8,39.7,38.1,38.0,26.5$, $26.4,25.5,25.4,22.8,22.6,19.6,19.4$.

IR v (cm-1): 3062 (w), 2946 (w), 2870 (w), 1689 (m), 1448 (w), 1423 (m), 1350 (w), 1302 (w), 1266 (m), 1195 (m), 1135 (w), 1098 (w), 1041 (w), 1002 (w), 909 (w), 731 (s), 699 (s).

HRMS(ESI) calcd for $\mathrm{C}_{15} \mathrm{H}_{18} \mathrm{NO}_{4}{ }^{+}(\mathrm{M}+\mathrm{H})$ 276.1236, found 276.1232.

Benzyl 7-(methoxy(methyl)carbamoyl)-2-azabicyclo[4.1.0]heptane-2-carboxylate (29) and benzyl 7-(1-methyl-1H-indole-2-carbonyl)-2-azabicyclo[4.1.0]heptane-2-carboxylate (14)

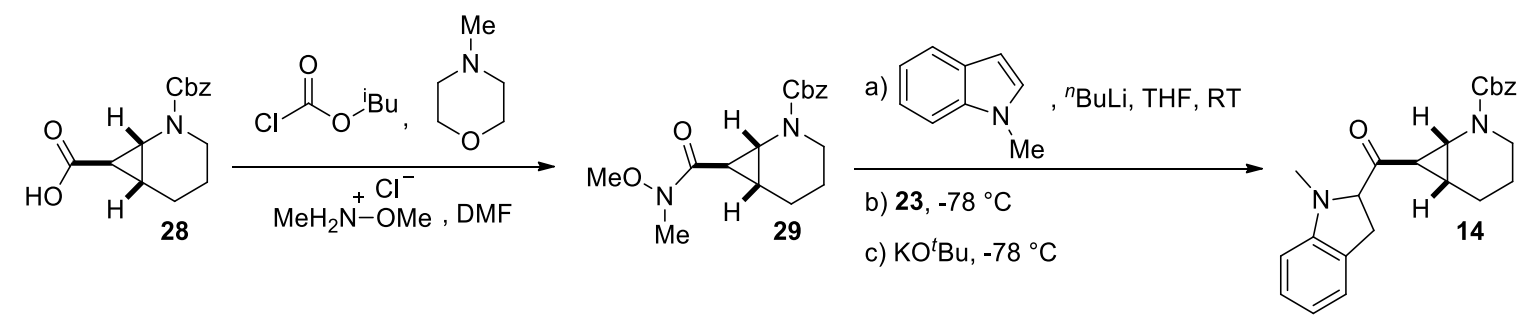

Following a reported procedure, ${ }^{[17]} \mathrm{N}$-methylmorpholine $(0.40 \mathrm{~mL}, 3.6 \mathrm{mmol}, 1.1$ equiv) was added to a solution of acid 28 ( $0.90 \mathrm{~g}, 3.3 \mathrm{mmol}, 1.0$ equiv) in DMF (3.3 mL) at $0{ }^{\circ} \mathrm{C}$. After 25

[17] S. R. Nagarajan, H. F. Lu, A. F. Gasiecki, I. K. Khanna, M. D. Parikh, B. N. Desai, T. E. Rogers, M. Clare, B. B. Chen, M. A. Russell, J. L. Keene, T. Duffin, V. W. Engleman, M. B. Finn, S. K. Freeman, J. A. Klover, G. 
min, isobutylchloroformate $\left(0.47 \mathrm{~mL}, 3.6 \mathrm{mmol}, 1.1\right.$ equiv) was added dropwise at $0{ }^{\circ} \mathrm{C}$. After $10 \mathrm{~min}, \mathrm{~N}, \mathrm{O}$-dimethylhydroxylamine hydrochloride $(0.35 \mathrm{~g}, 3.6 \mathrm{mmol}, 1.1$ equiv) was added, followed by $N$-methylmorpholine $(0.46 \mathrm{~mL}, 4.2 \mathrm{mmol}, 1.3$ equiv) and the reaction mixture was warmed to $23{ }^{\circ} \mathrm{C}$. After $12 \mathrm{~h}$, the reaction was quenched with $0.5 \mathrm{M} \mathrm{HCl}(6 \mathrm{~mL})$ and extracted with $\mathrm{CH}_{2} \mathrm{Cl}_{2}(3 \times 10 \mathrm{~mL})$. The combined organic layers were washed with $0.5 \mathrm{M} \mathrm{NaOH}(2 \times 10$ $\mathrm{mL})$, brine $(10 \mathrm{~mL})$, dried over $\mathrm{MgSO}_{4}$ and the solvent was removed under reduced pressure. The crude product was purified by flash column chromatography (PET/AcOEt 4:1-1:1) to yield Weinreb amide 29 (665 g, 2.09 mmol, 64\%, $\mathrm{R}_{\mathrm{f}}=0.30$ (PET/AcOEt 1:1) as a colorless oil, which was used directly in the next step.

$N$-Methyl indole $\left(0.16 \mathrm{~mL}, 1.2 \mathrm{mmol}, 1.2\right.$ equiv) was diluted in THF (4 mL) at $0{ }^{\circ} \mathrm{C}$ and ${ }^{n} \mathrm{BuLi}$ ( $0.47 \mathrm{~mL}, 1.2 \mathrm{mmol}, 1.2$ equiv) was added dropwise. The reaction mixture was stirred $1 \mathrm{~h}$ at $\mathrm{RT}$ and cooled to $-78{ }^{\circ} \mathrm{C}$. Weinreb amide 29 (dried through 3 co-evaporation with toluene, $0.31 \mathrm{~g}$, $0.97 \mathrm{mmol}, 1.0$ equiv) was added dropwise via cannula as a cooled $\left(-78^{\circ} \mathrm{C}\right)$ solution in THF $(2$ $\mathrm{mL})$. After further stirring $2 \mathrm{~h}$ at $-78{ }^{\circ} \mathrm{C}$, a solution of $\mathrm{KO} t \mathrm{Bu}(0.22 \mathrm{~g}, 2.0 \mathrm{mmol}, 2.0$ equiv) in THF ( $2 \mathrm{~mL}$ ) was added. After $2 \mathrm{~h}$, the reaction mixture was warmed to $0{ }^{\circ} \mathrm{C}$, whereas the yellow suspension became an orange solution. After $5 \mathrm{~min}$ at $0{ }^{\circ} \mathrm{C}$, the reaction mixture was quenched with sat. $\mathrm{NaHCO}_{3}(10 \mathrm{~mL})$ and extracted with $\mathrm{Et}_{2} \mathrm{O}(3 \times 10 \mathrm{~mL})$. The combined organic layers were washed with brine $(10 \mathrm{~mL})$, dried over $\mathrm{MgSO}_{4}$ and the solvent was removed under reduced pressure. The crude product was purified by flash column chromatography (PET/AcOEt 5:1-3:1) to yield indole 14 (251 mg, $0.646 \mathrm{mmol}, 67 \%)$ as a colorless solid.

$R_{f} 0.30$ (PET/AcOEt 3:1, Anisaldehyde).

$\operatorname{Mp} 119-121^{\circ} \mathrm{C}$.

${ }^{1} \mathrm{H} \mathrm{NMR}\left(\mathrm{CDCl}_{3}, 400 \mathrm{MHz}\right) \delta 7.70(\mathrm{~d}, J=8.0 \mathrm{~Hz}, 1 \mathrm{H} ; \mathrm{ArH}), 7.43-7.28$ (m, 4H; ArH), 7.22-7.14 (m, 1H; ArH), 7.10-7.02 (m, 2H; ArH), 6.99-6.89 (m, 2H; ArH), $5.16(\mathrm{~d}, J=12.5 \mathrm{~Hz}, 1 \mathrm{H}$; $\left.\mathrm{OCH}_{2}\right), 4.99\left(\mathrm{~d}, J=12.5 \mathrm{~Hz}, 1 \mathrm{H} ; \mathrm{OCH}_{2}\right), 4.06\left(\mathrm{~s}, 0.8 \mathrm{H} ; \mathrm{CH}_{3} \operatorname{rotamer} \mathrm{B}\right), 4.00\left(\mathrm{~s}, 2.2 \mathrm{H} ; \mathrm{CH}_{3}\right.$ rotamer A), $3.95\left(\mathrm{t}, J=3.8 \mathrm{~Hz}, 0.8 \mathrm{H}\right.$; $\mathrm{NCH}$ or $\mathrm{NCH}_{2}$ rotamer A), $3.84\left(\mathrm{~m}, 0.2 \mathrm{H} ; \mathrm{NCH}\right.$ or $\mathrm{NCH}_{2}$ rotamer B), $3.67\left(\mathrm{~m}, 0.2 \mathrm{H}\right.$; $\mathrm{NCH}$ or $\mathrm{NCH}_{2}$ rotamer B), $3.55(\mathrm{dd}, J=8.3 \mathrm{~Hz}, 2.2 \mathrm{~Hz}, 0.8 \mathrm{H}$; $\mathrm{NCH}$ or $\mathrm{NCH}_{2}$ rotamer A), $2.85\left(\mathrm{t}, J=12.5 \mathrm{~Hz}, 0.2 \mathrm{H} ; \mathrm{NCH}\right.$ or $\left.\mathrm{NCH}_{2} \operatorname{rotamer} \mathrm{B}\right), 2.76(\mathrm{td}, J=12.5$ $\mathrm{Hz}, 2.2 \mathrm{~Hz}, 0.8 \mathrm{H}$; $\mathrm{NCH}$ or $\mathrm{NCH}_{2}$ rotamer B), 2.63 (dd, J=5.8 Hz, 2.2 Hz, 1H; COCH), 2.28 (m, $1 \mathrm{H} ; \mathrm{CH}_{2} \mathrm{CH}$ ), 2.06-1.90 (m, 2H; $\left.\mathrm{CH}_{2}\right), 1.81-1.71\left(\mathrm{~m}, 1 \mathrm{H} ; \mathrm{CH}_{2}\right), 1.48\left(\mathrm{~m}, 1 \mathrm{H} ; \mathrm{CH}_{2}\right)$. 
${ }^{13} \mathrm{C} \mathrm{NMR}\left(\mathrm{CDCl}_{3}, 100 \mathrm{MHz}\right)$ (rotamers!) $\delta 189.9,156.4,140.0,136.2,135.4,128.4,128.0$, $127.5,127.5,126.8,125.9,125.7,125.5,122.8,122.6,120.6,120.3,111.3,111.1,110.2$, 67.1, 65.1, 41.6, 41.0, 33.5, 32.8, 32.0, 29.6, 23.8, 21.8, 19.9.

IR v (cm-1): $2941(\mathrm{w}), 2866$ (w), 1702 (s), 1639 (s), 1615 (w), 1513 (m), 1428 (s), 1407 (s), 1347 (s), 1299 (m), 1264 (m), 1195 (s), 1152 (m), 1129 (s), 1095 (m), 1034 (s), 908 (m), 794 (w), $769(\mathrm{w}), 752(\mathrm{~s}), 731(\mathrm{~s}), 698(\mathrm{~s}), 648(\mathrm{w})$.

HRMS(ESI) calcd for $\mathrm{C}_{24} \mathrm{H}_{25} \mathrm{~N}_{2} \mathrm{O}_{3}{ }^{+}(\mathrm{M}+\mathrm{H}) 389.1865$, found 389.1854.

Benzyl

7-methyl-6-oxo-2,3,4,4a,5,6,7,11c-octahydro- $1 H$-pyrido[3,2-c]carbazole-1carboxylate (32) and 7-methyl-3,4,4a,5,7,11c-hexahydro- $1 H$-pyrido[3,2-c]carbazol-6(2H)one (33)
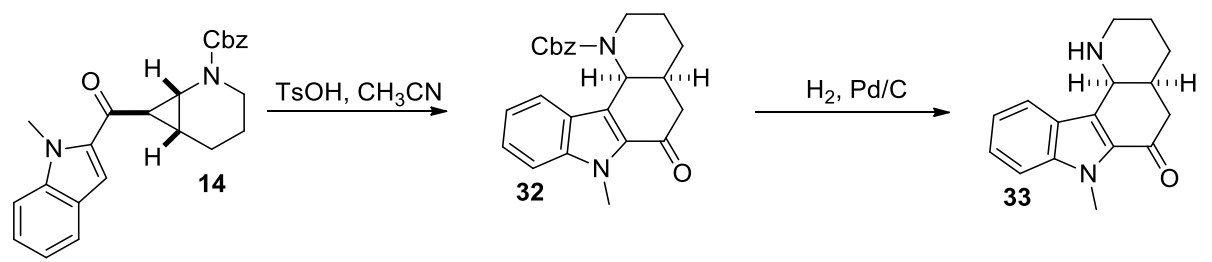

The reaction was performed following GP4, starting from cyclopropane 14 (50 mg, $0.13 \mathrm{mmol}$, 1.0 equiv) and tosic acid ( $4.9 \mathrm{mg}, 0.030 \mathrm{mmol}, 0.20$ equiv). The reaction was quenched after 12 h. Purification by flash chromatography (PET/AcOEt, 3:7) afforded 32 (45 mg, 0.12 mmol, 90 $\%$ ) as yellow oil. $R_{f} 0.60$ (PET/AcOEt 7:3, Anisaldehyde). The indole derivative 32 (35 mg, $0.090 \mathrm{mmol}, 1.0$ equiv) was deprotected following general procedure GP5, using Pd/C (10 mg, $10 \% \mathrm{w} / \mathrm{w})$ in $\mathrm{Et}_{2} \mathrm{O}(3.5 \mathrm{~mL})$ and $\mathrm{a}_{2}$ balloon. The suspension was filtered on celite and washed with DCM to afford $\mathbf{3 3}$ as yellow oil in quantitative yield (23 $\mathrm{mg}, 0.090 \mathrm{mmol}, 1.0$ equiv).

$R_{f} 0.80\left(\mathrm{DCM} / \mathrm{MeOH} / \mathrm{Et}_{3} \mathrm{~N} 3: 1: 2 \%\right.$, Anisaldehyde).

${ }^{1} \mathrm{H} \mathrm{NMR}\left(\mathrm{CDCl}_{3}, 400 \mathrm{MHz}\right) 7.88\left(\mathrm{~d}, J=8.1 \mathrm{~Hz}, 1 \mathrm{H} ; \mathrm{H}_{\mathrm{m}}\right.$ or $\left.\mathrm{H}_{\mathrm{h}}\right), 7.43-7.31\left(\mathrm{~m}, 2 \mathrm{H} ; \mathrm{H}_{\mathrm{m}}\right.$ or $\mathrm{H}_{\mathrm{h}}$ and $\mathrm{H}_{\mathrm{i}}$ or $\left.\mathrm{H}_{\mathrm{l}}\right), 7.18$ (t, $J=7.4 \mathrm{~Hz}, 1 \mathrm{H} ; \mathrm{H}_{\mathrm{i}}$ or $\left.\mathrm{H}_{\mathrm{l}}\right), 4.35\left(\mathrm{~d}, J=3.3 \mathrm{~Hz}, 1 \mathrm{H} ; \mathrm{H}_{\mathrm{f}}\right), 4.06\left(\mathrm{~s}, 3 \mathrm{H} ; 3 \mathrm{H}_{\mathrm{g}}\right), 3.23$ $\left(\mathrm{dd}, J=16.5 \mathrm{~Hz}, 12.0 \mathrm{~Hz}, 1 \mathrm{H} ; \mathrm{H}^{1}{ }_{\mathrm{e}}\right), 2.96\left(\mathrm{~m}, 1 \mathrm{H} ; \mathrm{H}^{1}{ }_{\mathrm{a}}\right), 2.83\left(\mathrm{~m}, 1 \mathrm{H} ; \mathrm{H}^{2}{ }_{\mathrm{a}}\right), 2.52\left(\mathrm{~m}, 1 \mathrm{H} ; \mathrm{H}_{\mathrm{d}}\right), 2.41$ (dd, $J=16.5 \mathrm{~Hz}, 4.0 \mathrm{~Hz}, 1 \mathrm{H} ; \mathrm{H}^{2}$ ) , 1.87-1.60 (m, $4 \mathrm{H} ; \mathrm{H}^{1}{ }_{\mathrm{c}}$ and/or $\mathrm{H}^{2}{ }_{\mathrm{c}}$ and/or $\mathrm{H}^{1}{ }_{\mathrm{b}}$ and/or $\mathrm{H}^{2}{ }_{\mathrm{b}}$ and $\mathrm{NH}), 1.57-1.45\left(\mathrm{~m}, 1 \mathrm{H} ; \mathrm{H}^{1}{ }_{\mathrm{b}}\right.$ or $\mathrm{H}^{2}{ }_{\mathrm{b}}$ or $\left.\mathrm{H}^{1}{ }_{\mathrm{c}}\right)$.

${ }^{13} \mathrm{C}$ NMR $\left(\mathrm{CDCl}_{3}, 100 \mathrm{MHz}\right) \delta 192.2,139.7,130.2,126.6,123.9,121.4$ 120.7, 110.3, 110.3, 51.4, 45.3, 41.0, 35.9, 31.5, 29.7, 28.5. 
IR v (cm-1): 3299 (w), 2926 (s), 2855 (m), 2149 (w), 1662 (s), 1616 (w), 1469 (m), 1432 (m), $1419(\mathrm{w}), 1386(\mathrm{w}), 1245(\mathrm{~m}), 1062(\mathrm{w}), 758(\mathrm{~s}), 746(\mathrm{~s}), 732(\mathrm{w}), 655(\mathrm{~m})$.

HRMS(ESI) calcd for $\mathrm{C}_{16} \mathrm{H}_{19} \mathrm{~N}_{2} \mathrm{O}^{+}(\mathrm{M}+\mathrm{H})$ 255.1497, found 255.1490.

Important correlations ROESY: $\mathrm{H}_{\mathrm{f}}-\mathrm{H}_{\mathrm{a}}{ }^{2} ; \mathrm{H}_{\mathrm{f}}-\mathrm{H}_{\mathrm{c}}{ }^{2} ; \mathrm{H}_{\mathrm{f}}-\mathrm{H}_{\mathrm{d}} ; \mathrm{H}_{\mathrm{f}}-\mathrm{H}_{\mathrm{e}}{ }^{2}$ (see the 2D spectra in section 6)

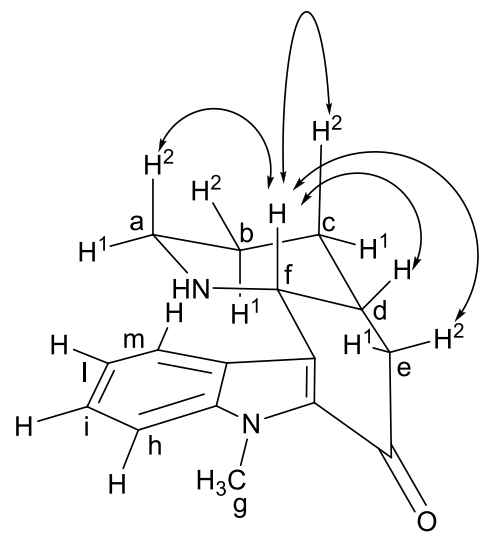

\section{3,4-Dihydro-2H-3-ethylpyridine-1-carboxylic acid benzyl ester (35)}

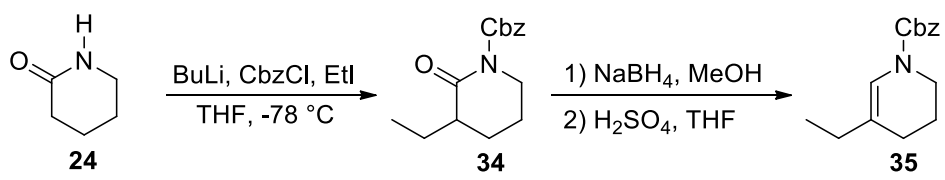

Following a reported procedure, ${ }^{[16]}$ a $2.5 \mathrm{M}$ solution of $n \mathrm{BuLi}$ in pentane $(88.0 \mathrm{~mL}, 220 \mathrm{mmol}$ 2.20 equiv) was added dropwise to a solution of $\delta$-valerolactam 24 (10.0 g, $100 \mathrm{mmol}, 1.00$ equiv) in $\mathrm{THF}(200 \mathrm{~mL})$ at $0^{\circ} \mathrm{C}$. The reaction mixture was stirred during $30 \mathrm{~min}$ and distilled ethyl iodide (12.2 mL, $150 \mathrm{mmol}, 1.50$ equiv) was added. The solution was stirred for additional 20 minutes before benzylchloroformate $(14.9 \mathrm{~mL}, 105 \mathrm{mmol}, 1.05$ equiv) in THF (50 mL) was added. The reaction was stirred further 20 minutes, diluted with ether $(250 \mathrm{~mL})$ and washed with brine $(2 \times 50 \mathrm{~mL})$. The organic layer was dried over magnesium sulfate and concentrated under reduced pressure. The crude product was purified by flash column chromatography (PET/AcOEt 5:1) to yield lactame $34(17.4 \mathrm{~g}, 66.5 \mathrm{mmol}, 67 \%)$ as colorless oil.

$R_{f} 0.25$ (PET/AcOEt 5:1, Anisaldehyde).

${ }^{1} \mathrm{H} \mathrm{NMR}\left(\mathrm{CDCl}_{3}, 400 \mathrm{MHz}\right) \delta 7.43(\mathrm{~m}, 2 \mathrm{H} ; \mathrm{ArH}), 7.39-7.28(\mathrm{~m}, 3 \mathrm{H} ; \mathrm{ArH}), 5.27$ (s, 2H; $\left.\mathrm{OCH}_{2}\right)$, 3.81 (m, 1H; $\left.\mathrm{NCH}_{2}\right), 3.69$ (m, 1H; $\left.\mathrm{NCH}_{2}\right), 2.35$ (m, 1H; CHCO), 2.07-1.73 (m, 4H; $\mathrm{CH}_{2} \mathrm{CH}_{2}$ ), 1.58-1.46 (m, 2H; $\left.\mathrm{CH}_{2} \mathrm{CH}_{3}\right), 0.96\left(\mathrm{t}, J=7.5 \mathrm{~Hz}, 3 \mathrm{H} ; \mathrm{CH}_{3}\right)$. 
Following a reported procedure,${ }^{[15]}$ sodium borohydride (829 mg, $21.9 \mathrm{mmol}, 1.05$ equiv) was added portionswise into a solution of lactame 34 (5.45 g, $20.9 \mathrm{mmol}, 1.00$ equiv) in methanol $(100 \mathrm{~mL})$ at $0{ }^{\circ} \mathrm{C}$. After the end of the addition, the reaction mixture was stirred at $0{ }^{\circ} \mathrm{C}$ for 15 min and poured onto ice-water $(150 \mathrm{~mL})$. The reaction mixture was extracted with AcOEt $(3 \times 100 \mathrm{~mL})$. The combined organic layers were washed with brine $(50 \mathrm{~mL})$, the combined water layers were extracted with AcOEt $(100 \mathrm{~mL})$ and the combined organic layers were dried over $\mathrm{MgSO}_{4}$ and the solvent was removed under reduced pressure. The residue was dissolved in $\mathrm{Et}_{2} \mathrm{O}$ $(50 \mathrm{~mL})$ and $0.5 \mathrm{~mL}$ of concentrated sulfuric acid was added dropwise. The reaction was stirred at RT for 1 hour then quenched with $\mathrm{K}_{2} \mathrm{CO}_{3}$ and dried on $\mathrm{Na}_{2} \mathrm{SO}_{4}$. The suspension was filtered and concentrated to afford 35 (4.75 g, $19.4 \mathrm{mmol}$, 93\% overall) without further purification as a colorless oil.

$R_{f} 0.36$ (PET/AcOEt 9:1, Anisaldehyde).

${ }^{1} \mathrm{H}$ NMR $\left(\mathrm{CDCl}_{3}, 400 \mathrm{MHz}\right) \delta$ 7.42-7.28 (m, 5H; ArH), 6.70 (s, 0.45H; alkeneH rotamer A), 6.60 (s, 0.55H; alkeneH rotamer B), $5.19\left(\mathrm{~s}, 1.1 \mathrm{H} ; \mathrm{OCH}_{2}\right.$ rotamer $\left.\mathrm{B}\right), 5.17\left(\mathrm{~s}, 0.9 \mathrm{H} ; \mathrm{OCH}_{2}\right.$ rotamer A), 3.57 (m, 2H; $\left.\mathrm{NCH}_{2}\right), 2.07-1.94\left(\mathrm{~m}, 4 \mathrm{H}\right.$; allylic $\left.\mathrm{CH}_{2}\right), 1.82\left(\mathrm{~m}, 2 \mathrm{H} ; \mathrm{CH}_{2} \mathrm{CH}_{2}\right), 1.06-0.97$ (m, $\left.3 \mathrm{H} ; \mathrm{CH}_{3}\right)$.

${ }^{13} \mathrm{C}$ NMR $\left(\mathrm{CDCl}_{3}, 100 \mathrm{MHz}\right)$ (rotamers!) $\delta 153.4,153.0,136.5,136.4,128.3,127.9,127.8$, 121.0, 120.6, 119.1, 118.7, 67.1, 67.0, 41.9, 41.8, 28.1, 28.1, 24.8, 24.7, 21.6, 21.6, 12.6, 12.4 .

IR v (cm-1): 2962 (w), 2934 (w), 2880 (w), 1703 (s), 1499 (w), 1409 (s), 1345 (m), 1313 (m), 1258 (s), 1202 (m), 1176 (m), 1111 (m), 1041 (m), 988 (m), 914 (m), 882 (m), 761 (m), 738 (m), $698(\mathrm{~m}), 635(\mathrm{~m}), 607(\mathrm{~m})$.

HRMS(ESI) calcd for $\mathrm{C}_{15} \mathrm{H}_{20} \mathrm{NO}_{2}{ }^{+}(\mathrm{M}+\mathrm{H})$ 246.1494, found 246.1496.

1-Benzyloxycarbonyl-5-ethyl-1-azabicyclo[4.1.0]heptan-7-carboxylic acid ethyl ester (36) 1Benzyloxycarbonyl-5-ethyl-1-azabicyclo[4.1.0]heptan-7-carboxylic acid (37)

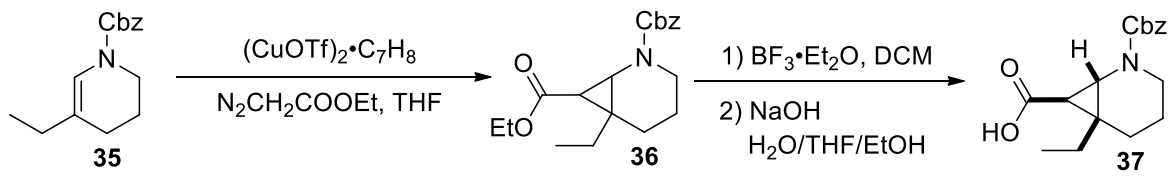


Following a slight modification of a reported procedure, ${ }^{[18]}$ a solution of ethyl diazoacetate $(6.2$ $\mathrm{mL}, 59 \mathrm{mmol}, 4.0$ equiv) in DCM (15 mL) was added to a solution of enamine 35 (3.64 g, 14.9 mmol, 1.00 equiv) and copper triflate (I) toluene complex (192 mg, $0.370 \mathrm{mmol}, 0.0200$ equiv) in DCM (15mL) over $18 \mathrm{~h}(1.3 \mathrm{~mL} / \mathrm{h})$ via syringe pump. After the addition was complete, the reaction was concentrated and purified by flash column chromatography (PET/AcOEt 15:1 until PET/AcOEt 9:1) to yield $36(3.74 \mathrm{~g}, 11.3 \mathrm{mmol}, 76 \%)$ as colorless oil. Following a reported procedure, ${ }^{[5]}$ the mixture of exo and endo esters $(1.50 \mathrm{~g}, 4.53 \mathrm{mmol}, 1.00$ equiv) in dichlorometane $(20 \mathrm{~mL})$ at $-20^{\circ} \mathrm{C}$ was treated with $\mathrm{BF}_{3} \bullet \mathrm{Et}_{2} \mathrm{O}(96 \mathrm{mg}, 0.68 \mathrm{mmol}, 0.15$ equiv). The reaction was allowed to warm at $0^{\circ} \mathrm{C}$ and stirred at the same temperature until the isomerization was finished (from $R_{f}$ 0.28-0.32 PET/AcOEt 9:1 to $R_{f}$ 0.30 PET/AcOEt 9:1, Anisaldehyde). Triethylamine ( $1 \mathrm{~mL})$ was added dropwise to the reaction and the mixture was diluted with $\mathrm{Et}_{2} \mathrm{O}(50 \mathrm{~mL})$ and washed with water and brine. The organic layer was dried over $\mathrm{Na}_{2} \mathrm{SO}_{4}$ and evaporated under reduced pressure to give a pale yellow oil ( $\left.1.50 \mathrm{~g}\right)$ which was used directly in the next step.

The crude oil (1.50 g, $4.53 \mathrm{mmol}, 1.00$ equiv) was dissolved in a solution of water/THF/EtOH $1 / 1 / 3$ (25 mL total) at $0^{\circ} \mathrm{C}$ and $\mathrm{NaOH}$ (1.63 g, $40.3 \mathrm{mmol}, 9.00$ equiv) was added portionswise. The reaction was heated to $60^{\circ} \mathrm{C}$ and stirred during 2 hours. The solution was concentrated, then diluted with water $(30 \mathrm{~mL})$ and washed with $\mathrm{Et}_{2} \mathrm{O}(3 \times 20 \mathrm{~mL})$. The aqueous layer was acidified with $\mathrm{HCl}$ (1 M aqueous solution) until $\mathrm{pH} 2$ and extracted with DCM (3x20 mL) to give 37 as a colorless oil which turns solid upon storage (1.25 g, $4.12 \mathrm{mmol}$, 91\% overall). No further purification was needed.

${ }^{1} \mathrm{H} \mathrm{NMR}\left(\mathrm{CDCl}_{3}, 400 \mathrm{MHz}\right) \delta$ 7.39-7.21 (m, 5H; $\left.\mathrm{ArH}\right), 5.23-5.09\left(\mathrm{~m}, 2 \mathrm{H} ; \mathrm{OCH}_{2}\right), 3.86(\mathrm{dt}, J=$ $12.5 \mathrm{~Hz}, 3.1 \mathrm{~Hz}, 0.7 \mathrm{H} ; \mathrm{NCH}_{2}$ rotamer A), $3.73\left(\mathrm{~m}, 0.3 \mathrm{H} ; \mathrm{NCH}_{2}\right.$ rotamer $\left.\mathrm{B}\right), 3.55$ (d, J = $3.5 \mathrm{~Hz}$, $0.3 \mathrm{H}$; NCH rotamer B), $3.49(\mathrm{~d}, J=3.6 \mathrm{~Hz}, 0.7 \mathrm{H}$; NCH rotamer A), $2.77(\mathrm{dt}, J=12.5 \mathrm{~Hz}, 1.7$ $\mathrm{Hz}, 0.3 \mathrm{H} ; \mathrm{NCH}_{2}$ rotamer B), $2.66\left(\mathrm{dt}, J=12.5 \mathrm{~Hz}, 3.4 \mathrm{~Hz}, 0.7 \mathrm{H} ; \mathrm{NCH}_{2}\right.$ rotamer A), 2.05 (m, 1H; CHCO), 1.93-1.12 (m, 6H; $\mathrm{CH}_{2}$ ), 0.99 (t, $\left.J=7.2 \mathrm{~Hz}, 1 \mathrm{H} ; \mathrm{CH}_{3} \operatorname{rotamer} \mathrm{B}\right), 0.93$ (t, $J=7.4 \mathrm{~Hz}$, $2 \mathrm{H} ; \mathrm{CH}_{3}$ rotamer $\left.\mathrm{A}\right)$.

${ }^{13} \mathrm{C}$ NMR $\left(\mathrm{CDCl}_{3}, 100 \mathrm{MHz}\right)$ (rotamers!) $\delta$ 177.2, 176.9, 156.2, 136.5, 128.5, 128.4, 128.1, 127.7, 127.2, 67.1, 67.0, 44.9, 44.8, 41.5, 41.1, 34.7, 34.0, 31.1, 30.6, 25.9, 25.7, 21.5, 21.3, 20.9, 10.3, 9.9.

[18] R. Beumer, C. Bubert, C. Cabrele, O. Vielhauer, M. Pietzsch, O. Reiser, J. Org. Chem. 2000, 65, 8960. 
IR v (cm-1): 2956 (w), 2939 (w), 2864 (w), 1704 (s), 1584 (w), 1456 (s), 1423 (s), 1348 (m), 1270 (m), 1240 (m), 1214 (s), 1129 (m), 1036 (m), 1017 (w), 948 (w), 909 (m), 883 (m), 752 (m), $736(\mathrm{~s}), 698(\mathrm{~m}), 676(\mathrm{~m}), 668(\mathrm{~m}), 635(\mathrm{~m})$.

HRMS(ESI) calcd for $\mathrm{C}_{17} \mathrm{H}_{22} \mathrm{NO}_{4}{ }^{+}(\mathrm{M}+\mathrm{H}) 304.1549$, found 304.1548.

\section{$N$-Methoxy- $N$-methylcarbamo-6-yl-5-ethyl-1-azabicyclo[4.1.0]heptanes-1- benzylcarboxylate (38)}

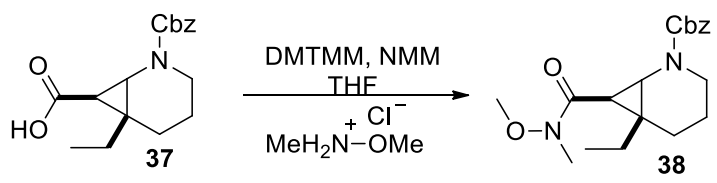

Dimethoxytriazin- $N$-methylmorpholinium chloride ${ }^{[19]}$ (DMTMM, $900 \mathrm{mg}, 3.24 \mathrm{mmol}, 1.50$ equiv) was suspended into a solution of acid $37(655 \mathrm{mg}, 2.16 \mathrm{mmol}, 1.00$ equiv) in THF (7.5 $\mathrm{mL}$ ) and the reaction mixture was stirred at RT during 60 min. $N, O$-dimethylhydroxylamine hydrochloride ( $97.5 \mathrm{mg}, 2.16 \mathrm{mmol}, 1.00$ equiv) was added, followed by $N$-methylmorpholine (475 $\mu \mathrm{L}, 4.32 \mathrm{mmol}, 2.00$ equiv) and the reaction mixture was stirred during 36 hours. The reaction was quenched with a $5 \%$ aqueous solution of citric acid and extracted with $\mathrm{Et}_{2} \mathrm{O}$. The combined organic layers were dried over $\mathrm{MgSO}_{4}$ and the solvent was removed under reduced pressure. The crude reaction mixture was purified by flash column chromatography (AcOEt/PET 3:7) to afford $\mathbf{3 8}$ (695 $\mathrm{mg}, 2.00 \mathrm{mmol}, 93 \%)$ as colorless oil.

$R_{f} 0.30$ (PET/AcOEt 7:3, Anisaldehyde).

${ }^{1} \mathrm{H}$ NMR $\left(\mathrm{CDCl}_{3}, 400 \mathrm{MHz}\right) \delta$ 7.35-7.19 (m, 5H; $\left.\mathrm{ArH}\right), 5.23\left(\mathrm{~d}, J=12.9 \mathrm{~Hz}, 0.8 \mathrm{H} ; \mathrm{OCH}_{2}\right.$ rotamer A), $5.14\left(\mathrm{~d}, J=12.5 \mathrm{~Hz}, 0.2 \mathrm{H} ; \mathrm{OCH}_{2}\right.$ rotamer B), $5.07\left(\mathrm{~d}, J=13.0 \mathrm{~Hz}, 1 \mathrm{H} ; \mathrm{OCH}_{2}\right), 3.84$ (dt, $J=12.5 \mathrm{~Hz}, 3.4 \mathrm{~Hz}, 0.8 \mathrm{H} ; \mathrm{NCH}_{2}$ rotamer A), $3.71\left(\mathrm{~m}, 0.2 \mathrm{H} ; \mathrm{NCH}_{2}\right.$, rotamer $\left.\mathrm{B}\right), 3.64$ (s, $0.6 \mathrm{H} ; \mathrm{OCH}_{3}$ rotamer $\left.\mathrm{B}\right), 3.58-3.52\left(\mathrm{~m}, 2.4 \mathrm{H} ; \mathrm{OCH}_{3}\right.$ rotamer $\mathrm{A}$ and $\mathrm{NCH}$ rotamer $\left.\mathrm{B}\right), 3.49(\mathrm{~d}, J=$ $3.7 \mathrm{~Hz}, 0.8 \mathrm{H}$; $\mathrm{NCH}$ rotamer A), $3.15\left(\mathrm{~s}, 0.6 \mathrm{H} ; \mathrm{NCH}_{3}\right.$ rotamer $\left.\mathrm{B}\right), 3.12\left(\mathrm{~s}, 2.4 \mathrm{H} ; \mathrm{NCH}_{3}\right.$ rotamer A), $2.73\left(\mathrm{t}, J=11.7 \mathrm{~Hz}, 0.2 \mathrm{H} ; \mathrm{NCH}_{2} \operatorname{rotamer} \mathrm{B}\right), 2.64\left(\mathrm{dt}, J=12.5 \mathrm{~Hz}, 2.2 \mathrm{~Hz}, 0.8 \mathrm{H} ; \mathrm{NCH}_{2}\right.$ rotamer A), 2.09-1.87 (m, 2H; $\mathrm{CHCO}$ and $\left.\mathrm{CH}_{2}\right), 1.77-1.54\left(\mathrm{~m}, 4 \mathrm{H} ; \mathrm{CH}_{2}\right), 1.36\left(\mathrm{~m}, 1 \mathrm{H} ; \mathrm{CH}_{2}\right)$, $0.85\left(\mathrm{t}, J=7.4 \mathrm{~Hz}, 3 \mathrm{H} ; \mathrm{CH}_{3}\right)$.

[19] M. Kunishima, C. Kawachi, F. Iwasaki, K. Terao, S. Tani, Tetrahedron Letters 1999, 40, 5327. 
${ }^{13} \mathrm{C} \mathrm{NMR}\left(\mathrm{CDCl}_{3}, 100 \mathrm{MHz}\right) \delta$ (rotamers!) 171.2, 156.2, 136.8, 128.2, 128.1, 127.8, 127.8, $127.4,127.0,66.8,66.5,61.2,42.9,42.7,41.5,41.0,33.0,32.4,29.1,28.5,26.0,25.9,25.2$, 21.6, 10.3.

IR v (cm-1): $2964(\mathrm{w}), 2937(\mathrm{w}), 2876$ (w), 1703 (s), 1651 (s), 1458 (m), 1417 (s), $1384(\mathrm{~m})$, 1358 (m), 1344 (m) (m), 1295 (m), 1266 (m), 1209 (m), 1180 (m), 1123 (m), 1016 (m), 913 (m), $769(\mathrm{w}), 734$ (s), $700(\mathrm{w})$.

HRMS(ESI) calcd for $\mathrm{C}_{19} \mathrm{H}_{27} \mathrm{~N}_{2} \mathrm{O}_{4}{ }^{+}(\mathrm{M}+\mathrm{H}) 347.1971$, found 347.1978.

6-(1-Methyl-1H-indol-2-carbonyl)-5-ethyl-1-azabicyclo[4.1.0]heptane-1-benzylcarboxylate (15)

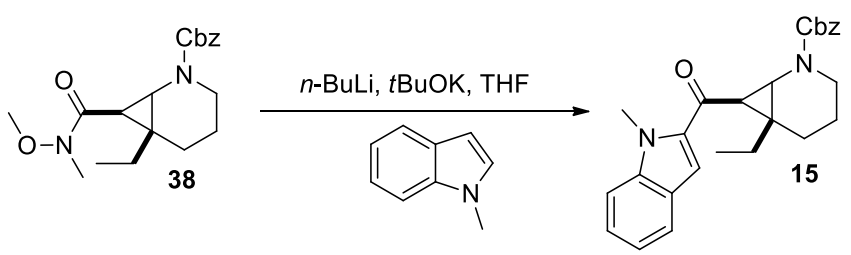

A solution of $N$-methylindole $\left(13 \mu \mathrm{L}, 0.10 \mathrm{mmol}, 1.3\right.$ equiv) in THF $(0.5 \mathrm{~mL})$ was stirred at $0^{\circ}$ C. Then a solution of $n \mathrm{BuLi}(2.5 \mathrm{M}$ in pentane, $38 \mu \mathrm{L}, 0.10 \mathrm{mmol}, 1.2$ equiv) diluted in THF (0.2 $\mathrm{mL}$ ) was added dropwise. The solution was warmed to RT, stirred for 1 hour and then cooled to $78^{\circ} \mathrm{C}$. From a separate flask, a solution of Weinreb amide 38 (27 mg, $0.080 \mathrm{mmol}, 1.0$ equiv) in THF $(0.4 \mathrm{~mL})$ was added via cannula into the solution. The mixture was stirred 2 hours at $-78^{\circ}$ $\mathrm{C}$, then a solution of $t \mathrm{BuOK}$ in THF $(0.3 \mathrm{~mL})$ was added dropwise. The reaction was stirred 2 hours at $-78^{\circ} \mathrm{C}$ and $5 \mathrm{~min}$ at $0^{\circ} \mathrm{C}$, quenched with $\mathrm{NaHCO}_{3}$ and extracted with $\mathrm{Et}_{2} \mathrm{O}(5 \times 3 \mathrm{~mL})$. The organic layers were dried over $\mathrm{Na}_{2} \mathrm{SO}_{4}$, evaporated on reduced pressure and purified on flash chromatography (AcOEt/PET 1:5) to give 15 (16 $\mathrm{mg}, 0.038 \mathrm{mmol} 48 \%$ ) as a yellow oil.

$R_{f} 0.60$ (PET/AcOEt 7:3, Anisaldehyde).

${ }^{1} \mathrm{H} \mathrm{NMR}\left(\mathrm{CDCl}_{3}, 400 \mathrm{MHz}\right) \delta 7.70(\mathrm{~d}, J=8.1 \mathrm{~Hz}, 1 \mathrm{H} ; \mathrm{ArH}), 7.45-7.29$ (m, 4H; ArH), 7.25-7.00 (m, 5H; ArH), 5.19 (d, J=12.7 Hz, 1H; $\left.\mathrm{OCH}_{2}\right), 5.04$ (d, J=12.7 Hz, 1H; $\left.\mathrm{OCH}_{2}\right), 4.08$ (s, 0.6H; $\mathrm{CH}_{3}$ rotamer B), $3.97\left(\mathrm{~s}, 2.4 \mathrm{H} ; \mathrm{CH}_{3} \operatorname{rotamer} \mathrm{A}\right), 3.95\left(\mathrm{dt}, J=13.2 \mathrm{~Hz}, 3.4 \mathrm{~Hz}, 0.8 \mathrm{H} ; \mathrm{NCH}_{2}\right.$, rotamer A), 3.88-3.82 (m, 0.2H; $\mathrm{NCH}_{2}$ rotamer B), $3.77(\mathrm{~d}, J=3.4 \mathrm{~Hz}, 1 \mathrm{H} ; \mathrm{NCH}), 2.83(\mathrm{t}, J=$ $11.9 \mathrm{~Hz}, 0.2 \mathrm{H} ; \mathrm{NCH}_{2}$, rotamer B), $2.74\left(\mathrm{dt}, J=12.0 \mathrm{~Hz}, 1.9 \mathrm{~Hz}, 0.8 \mathrm{H} ; \mathrm{NCH}_{2}\right.$, rotamer A), 2.68 $(\mathrm{d}, J=3.1 \mathrm{~Hz}, 0.2 \mathrm{H}$; COCH; rotamer B), $2.65(\mathrm{~d}, J=3.6 \mathrm{~Hz}, 0.8 \mathrm{H} ; \mathrm{COCH}$; rotamer A), 2.18 (m, $\left.1 \mathrm{H} ; \mathrm{CH}_{2}\right), 1.90-1.48\left(\mathrm{~m}, 5 \mathrm{H} ; \mathrm{CH}_{2}\right), 0.85\left(\mathrm{t}, J=7.4 \mathrm{~Hz}, 3 \mathrm{H} ; \mathrm{CH}_{3}\right)$. 
${ }^{13} \mathrm{C} \mathrm{NMR}\left(\mathrm{CDCl}_{3}, 100 \mathrm{MHz}\right)$ (rotamers!) $\delta 190.8,157.3,140.9,137.6,137.5,129.4,129.1$, 128.5, 128.2, 126.9, 126.6, 126.3, 123.7, 121.7, 111.7, 111.3, 67.9, 46.2, 45.9, 42.3, 42.6, 39.2, $38.8,38.4,33.0,27.3,27.0,26.0,23.0,22.811 .7,11.5$.

IR v (cm-1): $3064(\mathrm{w}), 3033(\mathrm{w}), 2938$ (w), $2876(\mathrm{w}), 1703$ (s), 1646 (s), $1614(\mathrm{w}), 1512(\mathrm{~m})$, 1464 (s), 1428 (s), 1408 (s), 1348 (s), 1268 (m), 1211 (s), 1196 (s), 1163 (m), 1129 (m), 1049 (m), 1027 (m), $910(\mathrm{~m}), 769(\mathrm{~m}), 737$ (s), $698(\mathrm{~m})$.

HRMS(ESI) calcd for $\mathrm{C}_{26} \mathrm{H}_{29} \mathrm{~N}_{2} \mathrm{O}_{3}{ }^{+}(\mathrm{M}+\mathrm{H})$ 417.2178, found 417.2181.

\section{(E)-2,4-Dimetoxy-cis-cinnamic acid (85)}
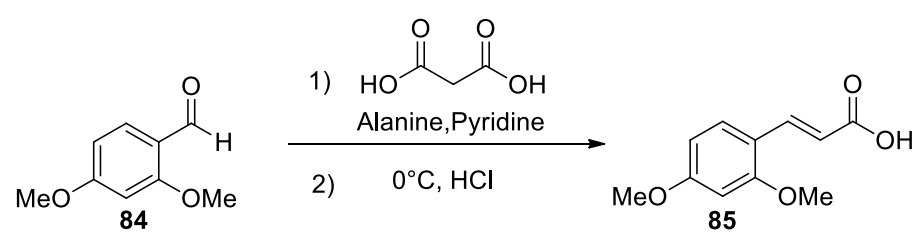

Following a reported procedure, ${ }^{[20]}$ a solution of aldehyde 84 (11.0 g, 66.7 mmol, 1.00 equiv), malonic acid (17.5 g, $168 \mathrm{mmol}, 2.50$ equiv) and $\beta$-alanine (1.0 g, $89 \mathrm{mmol}, 0.20$ equiv) in pyridine $(3 \mathrm{~mL})$ was stirred under reflux for $90 \mathrm{~min}$. After cooling to RT, the flask was transferred in an ice bath and a concentrated solution of $\mathrm{HCl}(8 \mathrm{~mL})$ was added dropwise. The precipitate was filtered, washed with cold water $(2 \times 10 \mathrm{~mL})$ and dried without further purification to give $\mathbf{8 5}$ as light yellow solid (12.5 g, $60.0 \mathrm{mmol}, 90 \%)$.

${ }^{1} \mathrm{H}$ NMR (DMSO-d $\left.6,400 \mathrm{MHz}\right) \delta 12.11(\mathrm{~s}, 1 \mathrm{H} ; \mathrm{OH}), 7.75(\mathrm{~d}, J=16.1 \mathrm{~Hz}, 1 \mathrm{H}$; alkeneH), 7.61 (d, $J=8.6 \mathrm{~Hz}, 1 \mathrm{H} ; \mathrm{ArH}), 6.64-6.54$ (m, 2H; ArH), 6.37 (d, $J=16.1 \mathrm{~Hz}, 1 \mathrm{H} ; \mathrm{CHCO}), 3.86$ (s, $\left.3 \mathrm{H} ; \mathrm{OCH}_{3}\right), 3.81$ (s, 3H; $\left.\mathrm{OCH}_{3}\right) .{ }^{1} \mathrm{H}$ NMR spectra corresponded to the literature values. ${ }^{[21]}$

(E)- $N$-Methoxy- $N$-methyl-3-(2,4-dimethoxyphenyl)-acrylamide (86) and $N$-methoxy- $N$ methyl-1-[2-(2,4-dimethoxyphenyl)-cyclopropan-1-yl]-formamide (87)

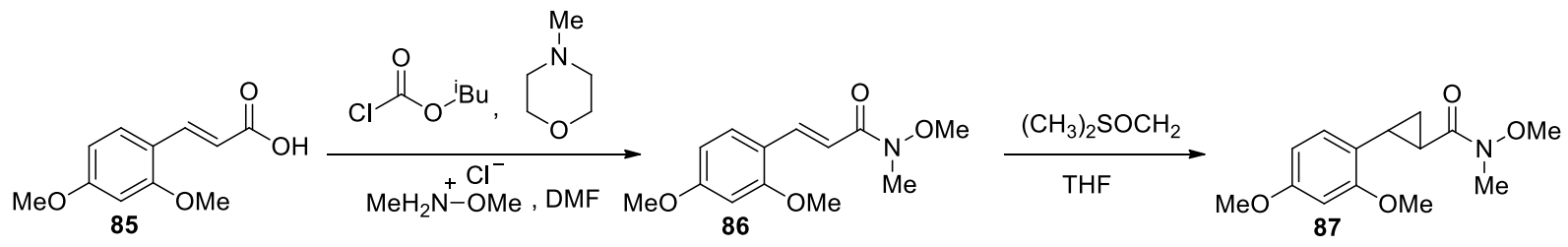

[20] Stabile, R. G.; Dicks, A. R. J. Chem. Educ. 2004, 81, 1488.

[21] Luadthong, C.; Tachaprutinun, A.; Wanichwecharungruang, S. P. Eur. Polym. J. 2008, 44, 1285. 
Following the reported procedure ${ }^{[22]} N$-methylmorpholine (7.23 mL, $65.9 \mathrm{mmol}, 1.10$ equiv) was added to a solution of acid 85 (12.47 g, $59.89 \mathrm{mmol}, 1.000$ equiv) in DMF (60 mL) at $0{ }^{\circ} \mathrm{C}$. After $25 \mathrm{~min}$, iso-butylchloroformate $\left(8.59 \mathrm{~mL}, 65.9 \mathrm{mmol} 1.10\right.$ equiv) was added dropwise at $0{ }^{\circ} \mathrm{C}$. After $10 \mathrm{~min}, \mathrm{~N}, \mathrm{O}$-dimethylhydroxylamine hydrochloride $(6.43 \mathrm{~g}, 65.9 \mathrm{mmol} 1.10$ equiv) was added, followed by $N$-methylmorpholine $(8.53 \mathrm{~mL}, 77.8 \mathrm{mmol}, 1.3$ equiv) and the reaction mixture was warmed to $23^{\circ} \mathrm{C}$. After $6 \mathrm{~h}$, the reaction was quenched with $0.5 \mathrm{M} \mathrm{HCl}(102 \mathrm{~mL})$ and extracted with $\mathrm{CH}_{2} \mathrm{Cl}_{2}(3 \times 120 \mathrm{~mL})$. The combined organic layers were washed with $0.5 \mathrm{M}$ $\mathrm{NaOH}(2 \times 120 \mathrm{~mL})$, brine $(2 \times 120 \mathrm{~mL})$, dried over $\mathrm{MgSO}_{4}$ and the solvent was removed under reduced pressure. After $30 \mathrm{~min}$ in high vacuum, the residues were dissolved in $\mathrm{Et}_{2} \mathrm{O}(360 \mathrm{~mL})$ and washed with brine $(2 \times 120 \mathrm{~mL})$, dried over $\mathrm{MgSO}_{4}$ and the solvent was removed under reduced pressure to give the Weinreb amide $\mathbf{8 6}$ which was used directly without purification.

A solution of ylide ${ }^{[23]}(53.4 \mathrm{~mL}, 28.8 \mathrm{mmol}, 1.20 \mathrm{eq})$ in anhydrous THF was added dropwise to a solution of the amide 86 (6.00 g, $23.9 \mathrm{mmol}, 1.00$ equiv) in THF (240 mL) at RT under nitrogen. The reaction was stirred at RT overnight then quenched with $\mathrm{NaHCO}_{3}(240 \mathrm{~mL})$ and extracted with $\mathrm{Et}_{2} \mathrm{O}(3 \times 240 \mathrm{~mL})$. The combined organic layers were washed with brine $(2 \times 240 \mathrm{~mL})$, dried over $\mathrm{MgSO}_{4}$ and the solvent was removed under reduced pressure. Purification by flash column chromatography (PET/AcOEt, 3:2) gave 87 (3.93 g, $14.8 \mathrm{mmol}, 62 \%)$ over 2 steps as white solid.

$R_{f} 0.35$ (PET/AcOEt 3:2, Anisaldehyde).

$\operatorname{Mp} 58-59^{\circ} \mathrm{C}$.

${ }^{1} \mathrm{H} \mathrm{NMR}\left(\mathrm{CDCl}_{3}, 400 \mathrm{MHz}\right) \delta 6.88(\mathrm{~d}, J=8.3 \mathrm{~Hz}, 1 \mathrm{H}$; ArH), $6.46-6.37$ (m, 2H; ArH), 3.80 (s, $\left.3 \mathrm{H} ; \mathrm{OCH}_{3}\right), 3.79$ (s, 3H; $\left.\mathrm{OCH}_{3}\right), 3.70\left(\mathrm{~s}, 3 \mathrm{H} ; \mathrm{OCH}_{3}\right), 3.24\left(\mathrm{~s}, 3 \mathrm{H} ; \mathrm{NCH}_{3}\right), 2.64-2.51(\mathrm{~m}, 1 \mathrm{H}$; CH-CH $), 2.25$ (m, 1H; $\left.\mathrm{CHCH}_{2}\right), 1.57-1.49\left(\mathrm{~m}, 1 \mathrm{H} ; \mathrm{CH}_{2} \mathrm{CH}\right), 1.30-1.20\left(\mathrm{~m}, 1 \mathrm{H} ; \mathrm{CH}_{2} \mathrm{CH}\right)$.

${ }^{13} \mathrm{C} \mathrm{NMR}\left(\mathrm{CDCl}_{3}, 100 \mathrm{MHz}\right) \delta 173.6,159.4,159.2,126.6,121.2,103.7,98.3,61.3,55.2,55.2$, $32.5,20.7,19.8,14.3$.

[22] Nagarajan, S. R.; Lu, H. F.; Gasiecki, A. F.; Khanna, I. K.; Parikh, M. D.; Desai, B. N.; Rogers, T. E.; Clare, M.; Chen, B. B.; Russell, M. A.; Keene, J. L.; Duffin, T.; Engleman, V. W.; Finn, M. B.; Freeman, S. K.; Klover, J. A.; Nickols, G. A.; Nickols, M. A.; Shannon, K. E.; Steininger, C. A.; Westlin, W. F.; Westlin, M. M.; Williams, M. L. Bioorg. Med. Chem. 2007, 15, 3390.

[23] $n$ BuLi (2.5 M, 1.0 equiv) was added dropwise to a solution of trimethylsufoxonium iodide (1.1 equiv) in anhydrous THF $(0.75 \mathrm{M})$ at $0^{\circ} \mathrm{C}$. The solution was allowed to warm to RT and stirring was continued under nitrogen for 1 hour. A solution $0.54 \mathrm{M}$ of ylide was obtained. 
IR v (cm-1): $3002(\mathrm{w}), 2960$ (w), 2938 (w), $2837(\mathrm{w}), 1650$ (s), 1614 (m), 1585 (m), 1510 (s), 1461 (s), 1438 (s), 1417 (s), 1394 (m), 1364 (m), 1321 (w), 1289 (s), 1263 (m), 1208 (s), 1176 (s), 1159 (s), 1155 (s), 1127 (s), 1096 (m), 1033 (s), 933 (m), 920 (m), 874 (w), 834 (s), 800 (w), $775(\mathrm{w}), 729(\mathrm{w})$.

HRMS(ESI) calcd for $\mathrm{C}_{14} \mathrm{H}_{20} \mathrm{NO}_{4}{ }^{+}(\mathrm{M}+\mathrm{H})$ 266.1387, found 266.1381.

\section{2-[2-(4-Methoxyphenyl)-1-cyclopropanecarbonyl]-indole (39)}

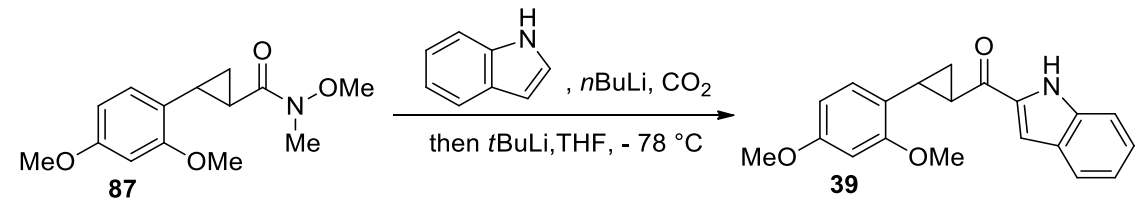

Following a slight modification of a reported procedure ${ }^{[24]}$ indole ( $66 \mathrm{mg}, 0.59 \mathrm{mmol}, 1.6$ equiv) was diluted in THF $(1.5 \mathrm{~mL})$ at $-70{ }^{\circ} \mathrm{C}$ and $n \mathrm{BuLi}(0.24 \mathrm{~mL}, 1.59 \mathrm{mmol}, 1.6$ equiv) was added dropwise. The reaction mixture was stirred at the same temperature for 30 min then $\mathrm{CO}_{2}$ was bubbled in during $20 \mathrm{~min}$. The solution was left to warm up to $\mathrm{rt}$ and the $\mathrm{CO}_{2}$ was removed in vacuo with a part of solvent. After the addition of freshly distilled THF (0.4 mL) the reaction was cooled down to $-78^{\circ} \mathrm{C}$. ${ }^{t} \mathrm{BuLi}(1.6 \mathrm{M}$ in pentane, $0.37 \mathrm{~mL}, 1.59 \mathrm{mmol}, 1.6$ equiv) was added dropwise during $10 \mathrm{~min}$ and the reaction was stirred $2 \mathrm{~h}$ at $-78^{\circ} \mathrm{C}$. Weinreb amide 87 (dried through 3 co-evaporation with toluene, $0.10 \mathrm{~g}, 0.38 \mathrm{mmol}, 1.0$ equiv) was added dropwise via cannula as a cooled $\left(-78^{\circ} \mathrm{C}\right)$ solution in THF $(0.4 \mathrm{~mL})$. After further stirring $1.5 \mathrm{~h}$ at $-78{ }^{\circ} \mathrm{C}$, the reaction mixture was transferred via cannula into a saturated aqueous solution of $\mathrm{NH}_{4} \mathrm{Cl}(20 \mathrm{~mL})$ at $0^{\circ} \mathrm{C}$ and stirred for 20 minutes before extraction with $\mathrm{Et}_{2} \mathrm{O}(3 \times 20 \mathrm{~mL})$. The combined organic layers were washed with brine $(10 \mathrm{~mL})$, dried over $\mathrm{MgSO}_{4}$ and the solvent was removed under reduced pressure. The crude product was purified by flash column chromatography (PET/AcOEt 9:1) to yield indole 39 (60 $\mathrm{mg}, 0.19 \mathrm{mmol}, 51 \%)$ as a white solid.

$R_{f} 0.25$ (PET/AcOEt 9:1, Anisaldehyde).

$\operatorname{Mp} 177-179^{\circ} \mathrm{C}$.

${ }^{1} \mathrm{H}$ NMR $\left(\mathrm{CDCl}_{3}, 400 \mathrm{MHz}\right) \delta 9.78(\mathrm{~s}, 1 \mathrm{H} ; \mathrm{NH}), 7.71(\mathrm{dd}, J=8.1,0.9 \mathrm{~Hz}, 1 \mathrm{H} ; \mathrm{ArH}), 7.49(\mathrm{dd}, J$ = 8.4, $0.9 \mathrm{~Hz}, 1 \mathrm{H} ; \operatorname{ArH}), 7.35(\mathrm{~m}, 1 \mathrm{H} ; \mathrm{ArH}), 7.30(\mathrm{dd}, J=2.1,0.9 \mathrm{~Hz}, 1 \mathrm{H} ; \operatorname{ArH}), 7.15(\mathrm{~m}, 1 \mathrm{H}$; ArH), 7.00 (m, 1H; ArH), 6.47 (m, 2H; ArH), 3.82 (s, 3H; OMe), 3.75 (s, 3H; OMe), 2.89 (ddd, 
$\left.J=9.0,7.1,4.1 \mathrm{~Hz}, 1 \mathrm{H} ; \mathrm{CHCH}_{2}\right), 2.75\left(\mathrm{~m}, 1 \mathrm{H} ; \mathrm{CHCH}_{2}\right), 1.91\left(\mathrm{~m}, 1 \mathrm{H} ; \mathrm{CH}_{2} \mathrm{CH}\right), 1.59(\mathrm{~m}, 1 \mathrm{H}$; $\mathrm{CH}_{2} \mathrm{CH}$ ).

${ }^{13} \mathrm{C}$ NMR $\left(\mathrm{CDCl}_{3}, 100 \mathrm{MHz}\right) \delta 191.6,159.7,159.5,137.4,136.0,127.7,127.0,126.0,122.9$, $121.1,120.7,112.3,109.2,103.8,98.4,55.4,55.4,28.1,24.9,17.2$.

IR v (cm-1): 3290 (m), 2957 (w), 2923 (w), 2853 (w), 1636 (s), 1617 (m), 1583 (w), 1520 (m), 1509 (m), 1465 (w), 1455 (w), 1437 (w), 1416 (w), 1402 (m), 1346 (w), 1330 (w), 1317 (w), 1289 (w), 1261 (m), 1231 (w), 1208 (s), 1182 (s), 1169 (m), 1160 (s), 1141 (m), 1120 (s), 1068 (m), 1048 (m), 1027 (m), 951 (w), 922 (w), 844 (m), 818 (w), 797 (m), 796 (m), 753 (m), 737 (s), $702(\mathrm{~m}), 687(\mathrm{~m}), 626(\mathrm{~m})$.

HRMS(ESI) calcd for $\mathrm{C}_{20} \mathrm{H}_{20} \mathrm{NO}_{3}{ }^{+}(\mathrm{M}+\mathrm{H}) 322.1443$, found 322.1444.

\section{4-(3,4-Dimethoxyphenyl)-2,3,4,9-tetrahydro-1H-carbazol-1-one (40)}

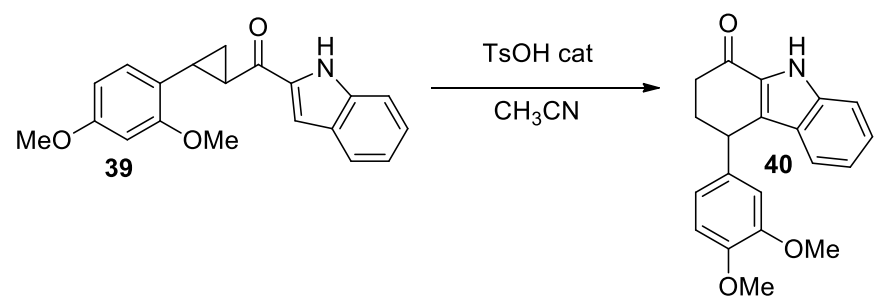

The reaction was performed following GP4, starting from cyclopropane 39 (45 mg, 0.14 mmol, 1.0 equiv) and tosic acid ( $5.4 \mathrm{mg}, 30 \mu \mathrm{mol}, 0.2$ equiv). The reaction was quenched after $20 \mathrm{~min}$ to give 40 (45 mg, $0.14 \mathrm{mmol}$, quantitative) as yellow solid without further purification.

$R_{f} 0.30$ (PET/AcOEt 7:3, Anisaldehyde).

$\operatorname{Mp} 183-185^{\circ} \mathrm{C}$.

${ }^{1} \mathrm{H}$ NMR $\left(\mathrm{CDCl}_{3}, 400 \mathrm{MHz}\right) \delta 8.81(\mathrm{~s}, 1 \mathrm{H} ; \mathrm{NH}), 7.41(\mathrm{~m}, 1 \mathrm{H} ; \mathrm{ArH}), 7.31(\mathrm{~m}, 1 \mathrm{H} ; \mathrm{ArH}), 7.11$ $(\mathrm{dd}, J=8.1,0.7 \mathrm{~Hz}, 1 \mathrm{H} ; \mathrm{ArH}), 6.96(\mathrm{~m}, 1 \mathrm{H} ; \mathrm{ArH}), 6.71$ (d, $J=8.4 \mathrm{~Hz}, 1 \mathrm{H} ; \mathrm{ArH}), 6.56(\mathrm{~d}, J=$ $2.4 \mathrm{~Hz}, 1 \mathrm{H}$; ArH), 6.31 (dd, $J=8.4,2.4 \mathrm{~Hz}, 1 \mathrm{H}$; $\mathrm{ArH}), 4.87\left(\mathrm{dd}, J=6.5,5.1 \mathrm{~Hz}, 1 \mathrm{H} ; \mathrm{CHCH}_{2}\right.$ ), 3.89 (s, 3H; OMe), 3.78 (s, 3H; OMe), 2.44-2.73 (m, 3H; $\left.\mathrm{CH}_{2}\right), 2.32\left(\mathrm{~m}, 1 \mathrm{H} ; \mathrm{CH}_{2}\right)$.

${ }^{13} \mathrm{C}$ NMR $\left(\mathrm{CDCl}_{3}, 100 \mathrm{MHz}\right) \delta 191.9,159.6,157.9,138.1,132.0,131.1,129.4,126.8,125.6$, $122.9,122.3,120.2,112.4,103.8,98.6,55.5,55.3,36.2,32.4,32.1$. 
IR v (cm-1): $3287(\mathrm{w}), 3280(\mathrm{w}), 3279(\mathrm{w}), 3274(\mathrm{w}), 2956(\mathrm{w}), 2927(\mathrm{w}), 1650$ (s), 1617 (m), 1616 (m), 1588 (w), 1537 (w), 1536 (w), 1505 (m), 1469 (m), 1440 (w), 1418 (w), 1334 (w), 1292 (m), 1257 (w), 1209 (m), 1167 (w), 1158 (w), 1116 (w), 1040 (w), 1040 (w), 912 (m), 834 (w), $826(\mathrm{w}), 735(\mathrm{~s})$.

HRMS(ESI) calcd for $\mathrm{C}_{20} \mathrm{H}_{20} \mathrm{NO}_{3}{ }^{+}(\mathrm{M}+\mathrm{H}) 322.1443$, found 322.1440.

\section{6-(1H-indol-2-carbonyl)-5-ethyl-1-azabicyclo[4.1.0]heptane-1-benzylcarboxylate (16)}

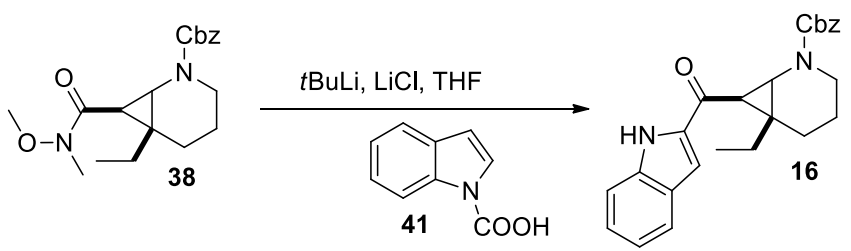

${ }^{t} \mathrm{BuLi}(1.6 \mathrm{M}$ in pentane, $38 \mu \mathrm{L}, 0.80 \mathrm{mmol}, 2.2$ equiv) was added dropwise into a solution of carboxylindole 41 prepared following GP5 (64 mg, $0.40 \mathrm{mmol}, 1.1$ equiv) and $\mathrm{LiCl}^{[25]}$ (17 mg, $0.40 \mathrm{mmol}, 1.1$ equiv) in THF $(1 \mathrm{~mL})$ at $-78^{\circ} \mathrm{C}$. The solution was stirred for 3 hours and then transferred via cannula into a solution of amide $\mathbf{3 8}$ (125 mg, $360 \mu \mathrm{mol}, 1.00$ equiv) in THF (1 $\mathrm{mL})$ at $-78^{\circ} \mathrm{C}$. The reaction was allowed to warm to $-20^{\circ} \mathrm{C}$ over 5 hours then transferred via cannula into saturated aqueous $\mathrm{NaHCO}_{3}$ solution $(10 \mathrm{~mL})$ at $0^{\circ} \mathrm{C}$. The aqueous phase was extracted with $\mathrm{Et}_{2} \mathrm{O}(5 \times 10 \mathrm{~mL})$. The organic layers were dried over $\mathrm{Na}_{2} \mathrm{SO}_{4}$, evaporated under reduced pressure and purified on flash chromatography with deactivated silica (AcOEt/PET 1:9) to give 16 (97 mg, $24 \mathrm{mmol}, 67 \%$ ) as yellow oil.

$R_{f} 0.70$ (PET/AcOEt 7:3, Anisaldehyde).

${ }^{1} \mathrm{H}$ NMR $\left(\mathrm{CDCl}_{3}, 400 \mathrm{MHz}\right) \delta 9.39(\mathrm{~d}, J=0.4 \mathrm{~Hz}, 0.2 \mathrm{H}$; NH rotamer B), $9.29(\mathrm{~m}, 0.8 \mathrm{H} ; \mathrm{NH}$ rotamer A), 7.72 (d, J = 8.0 Hz, 1H; ArH), 7.45 (m, 1H; ArH), 7.36 (m, 2H; ArH), 7.16 (m, 4H; $\mathrm{ArH}), 7.02(\mathrm{~m}, 2 \mathrm{H} ; \mathrm{ArH}), 5.21-5.08\left(\mathrm{~m}, 0.4 \mathrm{H} ; \mathrm{OCH}_{2}\right.$ rotamer $\left.\mathrm{B}\right), 5.15(\mathrm{~d}, J=12.7 \mathrm{~Hz}, 0.8 \mathrm{H}$; $\mathrm{OCH}_{2}$ rotamer A) $5.02\left(\mathrm{~d}, J=12.7 \mathrm{~Hz}, 0.8 \mathrm{H} ; \mathrm{OCH}_{2} \operatorname{rotamer} \mathrm{A}\right), 3.93\left(\mathrm{~m}, 1 \mathrm{H} ; \mathrm{NCH}_{2}\right), 3.81(\mathrm{~d}, J$ $=3.3 \mathrm{~Hz}, 1 \mathrm{H} ; \mathrm{NCH}), 2.84\left(\mathrm{t}, J=12.2 \mathrm{~Hz}, 0.2 \mathrm{H} ; \mathrm{NCH}_{2}\right.$ rotamer $\left.\mathrm{B}\right), 2.75(\mathrm{t}, J=12.1 \mathrm{~Hz}, 0.8 \mathrm{H}$; $\mathrm{NCH}_{2}$ rotamer A), $2.61(\mathrm{~d}, J=3.3 \mathrm{~Hz}, 1 \mathrm{H} ; \mathrm{COCH}), 2.15\left(\mathrm{~m}, 1 \mathrm{H} ; \mathrm{CH}_{2}\right), 1.77\left(\mathrm{~m}, 4 \mathrm{H} ; \mathrm{CH}_{2}\right), 1.54$ (m, $\left.1 \mathrm{H} ; \mathrm{CH}_{2}\right), 0.91\left(\mathrm{~m}, 0.6 \mathrm{H} ; \mathrm{CH}_{3}\right.$ rotamer $\left.\mathrm{B}\right), 0.82\left(\mathrm{t}, J=7.3 \mathrm{~Hz}, 2.4 \mathrm{H} ; \mathrm{CH}_{3}\right.$ rotamer A).

[25] $\mathrm{LiCl}$ was dried under $\mathrm{HV}(<0.05 \mathrm{Torr})$, warmed at $600^{\circ} \mathrm{C}(\mathrm{Mp})$ and stirred. It was then cooled down to RT and dissolved into $1 \mathrm{~mL}$ of dry THF. 
${ }^{13} \mathrm{C}$ NMR $\left(\mathrm{CDCl}_{3}, 100 \mathrm{MHz}\right)$ (rotamers!) $\delta$ 188.6, 156.3, 137.1, 136.6, 136.5, 128.5, 128.1, $128.1,127.7,127.5,127.3,126.1,122.9,122.7,120.9,120.7,112.1,108.6,108.4,66.9,46.0$, $45.6,41.7,41.3,38.3,36.7,36.0,26.3,26.1,25.0,21.9,21.8,10.7,10.6$.

IR v (cm-1): 3309 (w), 2960 (w), 2936 (w), $2876(\mathrm{w}), 1700$ (s), 1629 (s), $1521(\mathrm{~m}), 1446(\mathrm{~m})$, 1409 (s), 1349 (s), 1313 (w), 1299 (w), 1268 (m), 1232 (w), 1210 (m), 1192 (m), 1164 (m), 1140 (s), $1080(\mathrm{w}), 1034(\mathrm{w}), 1010(\mathrm{w}), 978(\mathrm{w}), 911(\mathrm{~m}), 799$ (w), $746(\mathrm{~s}), 736(\mathrm{~s}), 698(\mathrm{~m}), 606(\mathrm{w})$.

HRMS(ESI) calcd for $\mathrm{C}_{25} \mathrm{H}_{27} \mathrm{~N}_{2} \mathrm{O}_{3}{ }^{+}(\mathrm{M}+\mathrm{H})$ 403.2022, found 403.2034.

\section{3-Iodo-1-methyl-1H-indole (42)}

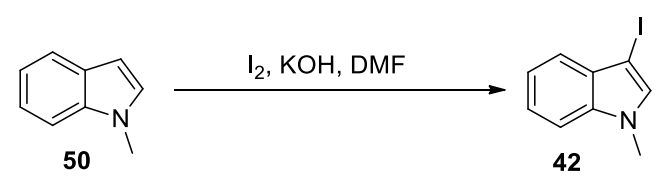

Following a reported procedure, ${ }^{[26]} \mathrm{I}_{2}(3.86 \mathrm{~g}, 15.2 \mathrm{mmol}, 2.00$ equiv) was added in one pot to a solution of $N$-methylindole $50(980 \mu \mathrm{L}, 7.60 \mathrm{mmol}, 1.00$ equiv) and $\mathrm{KOH}$ (1.60 g, $28.5 \mathrm{mmol}$, 3.75 equiv) in DMF $(20 \mathrm{~mL})$ at $\mathrm{rt}$. The reaction was stirred at the same temperature for 10 minutes then poured into a suspension of ice and water $(400 \mathrm{~mL})$ containing ammonia $(0.5 \%)$ and sodium metabisulphite $(0.1 \%)$. The emulsion was then extracted with a solution of hexane/ethyl acetate 1:1 (5x100 mL). The organic phase was washed with cold water, dried over $\mathrm{Na}_{2} \mathrm{SO}_{4}$ and evaporated under reduced pressure to afford iodoindole 42 (1.9 g, 74 mmol, 97\%). without further purification.

${ }^{1} \mathrm{H}$ NMR (DMSO-d $\left.6,400 \mathrm{MHz}\right) \delta 7.49(\mathrm{~m}, 1 \mathrm{H} ; \mathrm{ArH}), 7.44(\mathrm{~d}, J=8.2 \mathrm{~Hz}, 1 \mathrm{H} ; \mathrm{ArH}), 7.23(\mathrm{~m}$, 2H; $\mathrm{ArH}), 7.13$ (m, 1H; $\mathrm{ArH}), 3.77\left(\mathrm{~m}, 3 \mathrm{H} ; \mathrm{CH}_{3}\right)$.

${ }^{1} \mathrm{H}$ NMR spectra corresponded to the literature values. ${ }^{26}$

6-(1-Methyl-1H-indol-3-carbonyl)-5-ethyl-1-azabicyclo[4.1.0]heptane-1-benzylcarboxylate (17)

[26] Bocchi, V.; Palla, G., Synthesis 1982, 1096. 


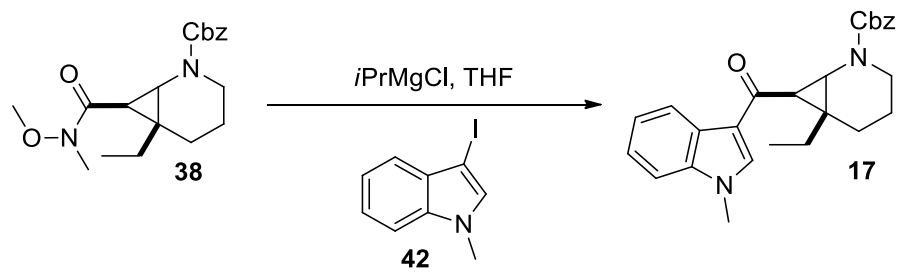

Following a slight modification of a reported procedure, ${ }^{[27]} i \mathrm{PrMgBr}$ was added dropwise into a solution of iodoindole 42 (165 mg, $64.0 \mathrm{mmol}, 3.00$ equiv) in THF $(2.5 \mathrm{~mL})$ at $-20^{\circ} \mathrm{C}$. The reaction was stirred $30 \mathrm{~min}$ at $-20^{\circ} \mathrm{C}$ then transferred via cannula into a solution of Weinreb amide 38 (74 mg, $0.21 \mathrm{mmol}, 1.0$ equiv) in THF $(2.5 \mathrm{~mL})$ at $0^{\circ} \mathrm{C}$. After $1 \mathrm{~h}$ at $0{ }^{\circ} \mathrm{C}$, the reaction was transferred via cannula into a saturated aqueous solution of $\mathrm{NaHCO}_{3}(10 \mathrm{~mL})$ at $0^{\circ} \mathrm{C}$ and stirred for 10 minutes before extraction with $\mathrm{Et}_{2} \mathrm{O}(3 \times 10 \mathrm{~mL})$. The combined organic layers were washed with brine $(10 \mathrm{~mL})$, dried over $\mathrm{MgSO}_{4}$ and the solvent was removed under reduced pressure. The crude product was purified by flash column chromatography (PET/AcOEt 8:2) to yield indole 17 (44 mg, $0.17 \mathrm{mmol}, 51 \%$ ) as a yellow oil.

$R_{f} 0.20$ (PET/AcOEt 8:2, Anisaldehyde).

${ }^{1} \mathrm{H}$ NMR $\left(\mathrm{CDCl}_{3}, 400 \mathrm{MHz}\right) \delta 8.38(\mathrm{~m}, 1 \mathrm{H} ; \mathrm{ArH}), 7.63$ (s, 1H; ArH), 7.39-7.28 (m, 4H; ArH), 7.20 (m, 2H; ArH), 7.09-6.98 (m, 2H; ArH), 5.17 (d, $\left.J=12.8 \mathrm{~Hz}, 1 \mathrm{H} ; \mathrm{OCH}_{2}\right), 5.03$ (d, $J=12.9$ $\left.\mathrm{Hz}, 1 \mathrm{H} ; \mathrm{OCH}_{2}\right), 3.92$ (m, 1H; $\left.\mathrm{NCH}_{2}\right), 3.84$ (s, 3H; $\left.\mathrm{NCH}_{3}\right), 3.80$ (d, J = 3.5 Hz, 1H; $\left.\mathrm{NCH}\right), 2.83$ $\left(\mathrm{t}, J=12.0,0.1 \mathrm{H} ; \mathrm{NCH}_{2}\right.$ rotamer B), $2.74\left(\mathrm{t}, J=12.4,0.9 \mathrm{H} ; \mathrm{NCH}_{2} \operatorname{rotamer} \mathrm{A}\right), 2.42(\mathrm{~d}, J=3.3$ $\mathrm{Hz}, 0.1 \mathrm{H}$; COCH rotamer B), 2.39 (d, J = 3.5 Hz, 0.9H; COCH rotamer A), $2.13\left(\mathrm{~m}, 1 \mathrm{H} ; \mathrm{CH}_{2}\right)$, 1.90-1.47 (m, 5H; $\left.\mathrm{CH}_{2}\right), 0.83\left(\mathrm{t}, J=7.3 \mathrm{~Hz}, 3 \mathrm{H} ; \mathrm{CH}_{3}\right)$.

${ }^{13} \mathrm{C} \mathrm{NMR}\left(\mathrm{CDCl}_{3}, 100 \mathrm{MHz}\right)$ (rotamers!) $\delta$ 191.0, 156.5, 137.4, 136.7, 134.8, 134.6, 128.4, $128.1,127.3,127.1,126.2,123.3,123.0,122.5,122.4,122.2,118.4,109.5,67.0,66.7,44.1$, $43.9,41.8,41.3,37.5,37.0,36.2,35.1,33.4,26.5,26.2,25.2,25.1,22.1,10.8$.

IR v (cm-1): 2938 (w), 2873 (w), 1701 (s), 1629 (m), 1531 (s), 1465 (s), 1410 (s), 1374 (m), 1358 (m), 1348 (m), 1297 (w), 1268 (w), 1215 (m), 1193 (w), 1180 (w), 1144 (w), 1127 (m), $1104(w), 1089$ (s), 1052 (w), $1012(w), 998$ (w), $914(w), 770(w), 745$ (s), 737 (s).

HRMS(ESI) calcd for $\mathrm{C}_{26} \mathrm{H}_{29} \mathrm{~N}_{2} \mathrm{O}_{3}{ }^{+}(\mathrm{M}+\mathrm{H})$ 417.2178, found 417.2181.

[27] Sapountzis, I.; Lin, W. W.; Kofink, C. C.; Despotopoulou, C.; Knochel, P., Angew. Chem, Int. Ed. 2005, 44, 1654. 


\section{Benzyl 6-ethyl-7-(1H-pyrrole-2-carbonyl)-2-azabicyclo[4.1.0]heptane-2-carboxylate (18)}

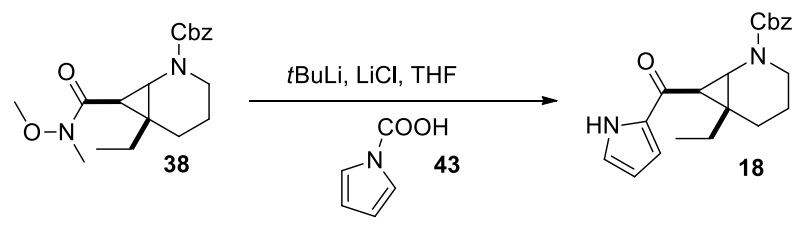

$t$ BuLi (0.94 mL, $1.5 \mathrm{mmol}, 2.2$ equiv) was added dropwise into a solution of $N$-carboxypyrrole 43 prepared following GP6 (84 mg, $0.75 \mathrm{mmol}, 1.1$ equiv) and $\mathrm{LiCl}$ (32 mg, $0.75 \mathrm{mmol}, 1.1$ equiv) in THF $(3 \mathrm{~mL})$ at $-78^{\circ} \mathrm{C}$. The reaction mixture was stirred $3 \mathrm{~h}$ at $-78{ }^{\circ} \mathrm{C}$ then added dropwise via cannula into a solution of Weinreb amide 38 ( $0.24 \mathrm{~g}, 0.68 \mathrm{mmol}, 1.0$ equiv) in THF ( $3 \mathrm{~mL}$ ) at $0{ }^{\circ} \mathrm{C}$ during 20 minutes. The reaction mixture was left to warm up to $-20{ }^{\circ} \mathrm{C}$ and stirred for additional 20 minutes. After that, the reaction was transferred via cannula into a saturated aqueous solution of $\mathrm{NaHCO}_{3}(15 \mathrm{~mL})$ at $0^{\circ} \mathrm{C}$ and stirred for 20 minutes and extracted with $\mathrm{Et}_{2} \mathrm{O}$ $(3 \times 10 \mathrm{~mL})$. The combined organic layers were washed with brine $(10 \mathrm{~mL})$, dried over $\mathrm{MgSO}_{4}$ and the solvent was removed under reduced pressure. The crude product was purified by flash column chromatography (AcOEt/PET 2:8 on deactivated silica) to yield pyrrole 18 (58 mg, 0.16 mmol, 24\%) as a yellowish oil.

$R_{f} 0.4$ (PET/AcOEt 7:3, Anisaldehyde).

${ }^{1} \mathrm{H}$ NMR $\left(\mathrm{CDCl}_{3}, 400 \mathrm{MHz}\right) \delta 9.77(\mathrm{~s}, 0.3 \mathrm{H} ; \mathrm{NH}), 9.56(\mathrm{~s}, 0.7 \mathrm{H} ; \mathrm{NH}), 7.34(\mathrm{~m}, 1.5 \mathrm{H} ; \operatorname{ArH}$ rotamer B), 7.23-7.07 (m, 3.5H; ArH rotamer A), $7.01(\mathrm{~m}, 0.7 \mathrm{H}$; pyrroleH rotamer A), 6.97 (m, 0.3H; pyrroleH rotamer B), $6.87(\mathrm{~m}, 1 \mathrm{H}$; pyrroleH), $6.29(\mathrm{~m}, 0.7 \mathrm{H}$; pyrroleH rotamer $\mathrm{A}), 6.25$ (s, $0.3 \mathrm{H}$; CH-pyrrole rotamer B), 5.17 (d, $\left.J=12.8 \mathrm{~Hz}, 0.7 \mathrm{H} ; \mathrm{OCH}_{2} \operatorname{rotamer} \mathrm{A}\right), 5.09$ (d, $J=12.5$ $\mathrm{Hz}, 0.3 \mathrm{H} ; \mathrm{OCH}_{2}$ rotamer B), $5.04\left(\mathrm{~d}, J=12.8 \mathrm{~Hz}, 0.7 \mathrm{H} ; \mathrm{OCH}_{2} \operatorname{rotamer} \mathrm{A}\right), 4.98(\mathrm{~d}, J=12.5 \mathrm{~Hz}$, $0.3 \mathrm{H} ; \mathrm{OCH}_{2}$ rotamer B), $3.91\left(\mathrm{~m}, 1 \mathrm{H} ; \mathrm{NCH}_{2}\right), 3.78(\mathrm{~d}, J=3.8 \mathrm{~Hz}, 0.3 \mathrm{H}$; $\mathrm{NCH}$ rotamer B), 3.72 $\left(\mathrm{d}, J=3.6 \mathrm{~Hz}, 0.8 \mathrm{H} ; \mathrm{NCH}\right.$ rotamer A), $2.80\left(\mathrm{~m}, 0.3 \mathrm{H} ; \mathrm{NCH}_{2} \operatorname{rotamer} \mathrm{B}\right), 2.71\left(\mathrm{~m}, 0.7 \mathrm{H} ; \mathrm{NCH}_{2}\right.$ rotamer A), $2.40(\mathrm{~d}, J=3.6 \mathrm{~Hz}, 1 \mathrm{H} ; \mathrm{COCH}), 2.09\left(\mathrm{~m}, 1 \mathrm{H} ; \mathrm{CH}_{2}\right), 1.86-1.62\left(\mathrm{~m}, 4 \mathrm{H} ; \mathrm{CH}_{2}\right), 1.50$ $\left(\mathrm{m}, 1 \mathrm{H} ; \mathrm{CH}_{2}\right), 0.82\left(\mathrm{t}, J=7.4 \mathrm{~Hz}, 3 \mathrm{H} ; \mathrm{CH}_{2}\right)$.

${ }^{13} \mathrm{C} \mathrm{NMR}\left(\mathrm{CDCl}_{3}, 100 \mathrm{MHz}\right)$ (rotamers!) $\delta 186.3,156.3,136.7,133.4,128.4,128.2,128.1$, 128.0, 127.5, 127.1, 123.8, 123.7, 115.4, 115.3, 110.6, 110.4, 67.2, 66.8, 45.1, 44.7, 41.7, 41.2, $37.0,36.0,35.4,26.3,26.0,25.2,25.0,21.9,21.8,19.4,10.7,10.6$.

IR v (cm-1): 3276 (w), $3266(w), 3242(w), 2962(w), 2956(w), 2949(w), 2936(w), 2876(w)$, 1704 (s), 1619 (m), 1544 (w), 1498 (w), 1457 (m), 1428 (s), 1414 (s), 1351 (m), 1323 (m), 1294 
(m), 1267 (m), 1209 (m), 1194 (w), 1193 (w), 1181 (w), 1115 (s), 1061 (m), 1040 (w), 1039 (w), $1029(w), 1011(w), 974(w), 939(w), 911(w), 902(w), 901(w), 885(w), 848(w), 833(w), 781$ (m), $767(\mathrm{~m}), 746(\mathrm{~m}), 699(\mathrm{~m}), 647(\mathrm{w})$.

HRMS(ESI) calcd for $\mathrm{C}_{21} \mathrm{H}_{25} \mathrm{~N}_{2} \mathrm{O}_{3}{ }^{+}(\mathrm{M}+\mathrm{H}) 353.1865$, found 353.1861 .

\section{Benzyl 7-(3,4-dihydro-2H-pyran-6-carbonyl)-6-ethyl-2 -azabicyclo[4.1.0] heptane -2- carboxylate (19)}
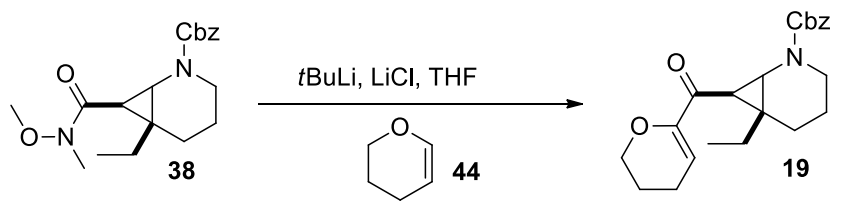

A solution of dihydropyran $44(33 \mu \mathrm{L}, 0.37 \mathrm{mmol}, 1.3$ equiv) and $\mathrm{LiCl}$ (30 mg, $0.58 \mathrm{mmol}, 2.0$ equiv) in THF $(1.5 \mathrm{~mL})$ was stirred at $-78^{\circ} \mathrm{C}$. Then a solution of $t \mathrm{BuLi}(1.6 \mathrm{M}$ in hexane, 0.22 $\mathrm{mL}, 0.35 \mathrm{mmol}, 1.2$ equiv) was added dropwise. The solution was warmed to $0^{\circ} \mathrm{C}$ and stirred for 40 minutes before cooling down again at $-78^{\circ} \mathrm{C}$. The lithiated dihydropyran was then added via cannula into the solution of Weinreb amide 38 ( $0.10 \mathrm{~g}, 0.29 \mathrm{mmol}, 1.0$ equiv) in THF (1.5 mL) at $0^{\circ} \mathrm{C}$ during 20 minutes. The mixture was stirred for additional 20 minutes, transferred via cannula into a saturated aqueous solution of $\mathrm{NaHCO}_{3}(5 \mathrm{~mL})$ at $0^{\circ} \mathrm{C}$ and extracted with $\mathrm{Et}_{2} \mathrm{O}$ $(5 \times 3 \mathrm{~mL})$. The organic layers were dried over $\mathrm{Na}_{2} \mathrm{SO}_{4}$, evaporated on reduced pressure and purified on flash chromatography (AcOEt/PET 2:8 on deactivated silica) to give 19 (30 mg, 81 $\mu \mathrm{mol}, 29 \%$ ) as a yellow oil.

$R_{f} 0.6$ (PET/AcOEt 7:3, Anisaldehyde).

${ }^{1} \mathrm{H}$ NMR $\left(\mathrm{CDCl}_{3}, 400 \mathrm{MHz}\right) \delta$ 7.39-7.23 (m, 5H; ArH), $5.88\left(\mathrm{t}, J=4.2 \mathrm{~Hz}, 1 \mathrm{H} ; \mathrm{CHCH}_{2}\right), 5.19$ $\left(\mathrm{d}, J=12.8 \mathrm{~Hz}, 1 \mathrm{H} ; \mathrm{PhCH}_{2}\right), 5.08$ (d, $\left.J=12.8 \mathrm{~Hz}, 1 \mathrm{H} ; \mathrm{PhCH}_{2}\right), 4.16-3.98$ (m, 2H; $\mathrm{OCH}_{2}$ rotamer A and rotamer B), $3.86\left(\mathrm{dt}, J=12.9,3.2 \mathrm{~Hz}, 0.8 \mathrm{H} ; \mathrm{NCH}_{2} \operatorname{rotamer} \mathrm{A}\right), 3.72(\mathrm{~m}, 0.2 \mathrm{H}$; $\mathrm{NCH}_{2}$ rotamer B), $3.70(\mathrm{~d}, J=3.5 \mathrm{~Hz}, 0.2 \mathrm{H}$; $\mathrm{NCH}$ rotamer $\mathrm{B}), 3.64(\mathrm{~d}, J=3.7 \mathrm{~Hz}, 0.8 \mathrm{H}$; $\mathrm{NCH}$ rotamer A), $2.75\left(\mathrm{~m}, 0.2 \mathrm{H} ; \mathrm{NCH}_{2}\right.$ rotamer $\left.\mathrm{B}\right), 2.67\left(\mathrm{~m}, 0.8 \mathrm{H} ; \mathrm{NCH}_{2}\right.$ rotamer $\left.\mathrm{A}\right), 2.46(\mathrm{~d}, J=3.7$ $\mathrm{Hz}, 1 \mathrm{H} ; \mathrm{COCH}), 2.19\left(\mathrm{~m}, 2 \mathrm{H} ; \mathrm{CH}_{2} \mathrm{CH}\right), 2.07\left(\mathrm{~m}, 1 \mathrm{H} ; \mathrm{CH}_{2}\right), 1.84\left(\mathrm{~m}, 2 \mathrm{H} ; \mathrm{CH}_{2}\right), 1.78-1.41(\mathrm{~m}$, $\left.5 \mathrm{H} ; \mathrm{CH}_{2}\right), 0.81\left(\mathrm{t}, J=7.3 \mathrm{~Hz}, 3 \mathrm{H} ; \mathrm{CH}_{3}\right)$. 
${ }^{13} \mathrm{C} \mathrm{NMR}\left(\mathrm{CDCl}_{3}, 100 \mathrm{MHz}\right)$ (rotamers!) $\delta$ 192.8, 169.6, 151.9, 128.4, 128.3, 128.0, 128.0, 127.5, 127.5, 127.3, 127.2, 127.0, 108.0, 66.8, 66.3, 46.0, 41.2, 38.1, 34.8, 26.2, 24.6, 21.8, 21.6, 20.7, 10.8 .

IR v (cm-1): $2956(\mathrm{w}), 2935(\mathrm{w}), 2876(\mathrm{w}), 1705(\mathrm{~s}), 1678(\mathrm{~m}), 1626(\mathrm{~m}), 1498(\mathrm{w}), 1447(\mathrm{~m})$, 1429 (m), 1406 (s), 1369 (m), 1349 (m), 1309 (m), 1287 (m), 1268 (m), 1239 (w), 1213 (m), 1198 (m), 1170 (m), 1122 (m), 1092 (m), 1061 (s), 1027 (m), 1011 (m), 981 (w), 973 (w), 918 (m), $901(\mathrm{w}), 873(\mathrm{w}), 768(\mathrm{~m}), 739(\mathrm{~m}), 699(\mathrm{~m}), 630(\mathrm{w})$.

HRMS(ESI) calcd for $\mathrm{C}_{22} \mathrm{H}_{28} \mathrm{NO}_{4}{ }^{+}(\mathrm{M}+\mathrm{H}) 370.2018$, found 370.2033

Benzyl 6-ethyl-7-(thiophene-2-carbonyl)-2-azabicyclo[4.1.0]heptane-2-carboxylate (20)

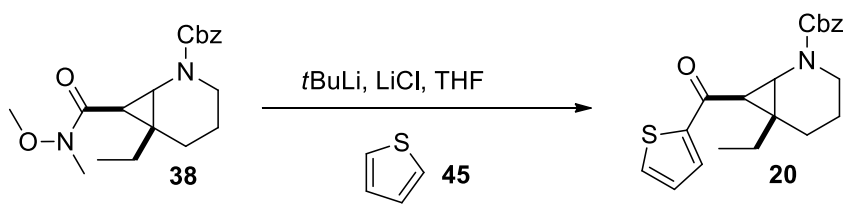

A solution of thiophene $45(30 \mu \mathrm{L}, 0.37 \mathrm{mmol}, 1.3$ equiv) and $\mathrm{LiCl}$ (30 mg, $0.58 \mathrm{mmol}, 2.0$ equiv) in THF $(1.5 \mathrm{~mL})$ was stirred at $-78^{\circ} \mathrm{C}$. Then a solution of $t \mathrm{BuLi}(1.6 \mathrm{M}$ in hexane, 0.22 $\mathrm{mL}, 0.35 \mathrm{mmol}, 1.2$ equiv) was added dropwise. The solution was warmed to $0^{\circ} \mathrm{C}$ and stirred for 40 minutes before cooling down again at $-78^{\circ} \mathrm{C}$. The lithiated thiophene was then added via cannula into the solution of Weinreb amide 38 ( $0.10 \mathrm{~g}, 0.29 \mathrm{mmol}, 1.0$ equiv) in THF (1.5 mL) at $0^{\circ} \mathrm{C}$ during 20 minutes. The mixture was stirred for additional 20 minutes, transferred via cannula into a saturated aqueous solution of $\mathrm{NaHCO}_{3}(5 \mathrm{~mL})$ at $0^{\circ} \mathrm{C}$ and extracted with $\mathrm{Et}_{2} \mathrm{O}$ $(5 \times 3 \mathrm{~mL})$. The organic layers were dried over $\mathrm{Na}_{2} \mathrm{SO}_{4}$, evaporated under reduced pressure and purified on flash chromatography (AcOEt/PET 2:8 on deactivated silica) to give $\mathbf{2 0}$ (27 $\mathrm{mg}, 73$ $\mu \mathrm{mol}, 25 \%$ ) as a yellow oil.

$R_{f} 0.3$ (PET/AcOEt 8:2, Anisaldehyde).

${ }^{1} \mathrm{H} \mathrm{NMR}\left(\mathrm{CDCl}_{3}, 400 \mathrm{MHz}\right) \delta 7.69(\mathrm{~d}, J=0.9 \mathrm{~Hz}, 0.2 \mathrm{H}$; thiopheneH rotamer $\mathrm{B}), 7.66(\mathrm{dd}, J=$ 3.5, $0.7 \mathrm{~Hz}, 0.8 \mathrm{H}$; thiopheneH rotamer A), $7.61(\mathrm{dd}, J=4.8,0.7 \mathrm{~Hz}, 0.8 \mathrm{H}$; thiopheneH rotamer A), $7.58(\mathrm{~d}, J=0.9 \mathrm{~Hz}, 0.2 \mathrm{H}$; thiopheneH rotamer B), $7.33(\mathrm{~m}, 1 \mathrm{H}$; thiopheneH), 7.23-7.08 (m, $5 \mathrm{H} ; \mathrm{ArH}), 5.19\left(\mathrm{~d}, J=12.8 \mathrm{~Hz}, 0.8 \mathrm{H} ; \mathrm{OCH}_{2} \operatorname{rotamer} \mathrm{A}\right), 5.18\left(\mathrm{~d}, J=12.0 \mathrm{~Hz}, 0.2 \mathrm{H} ; \mathrm{OCH}_{2}\right.$ rotamer B), $5.08\left(\mathrm{~d}, J=12.5 \mathrm{~Hz}, 0.2 \mathrm{H} ; \mathrm{OCH}_{2} \operatorname{rotamer} \mathrm{B}\right), 5.04\left(\mathrm{~d}, J=12.8 \mathrm{~Hz}, 0.8 \mathrm{H} ; \mathrm{OCH}_{2}\right.$ rotamer A), $3.91\left(\mathrm{~m}, 0.8 \mathrm{H} ; \mathrm{NCH}_{2}\right.$ rotamer $\left.\mathrm{A}\right), 3.85\left(\mathrm{~m}, 0.2 \mathrm{H} ; \mathrm{NCH}_{2}\right.$ rotamer $\left.\mathrm{B}\right), 3.78(\mathrm{~d}, J=3.5$ 
$\mathrm{Hz}, 1 \mathrm{H} ; \mathrm{NCH}), 2.82\left(\mathrm{~m}, 0.2 \mathrm{H} ; \mathrm{NCH}_{2}\right.$ rotamer $\left.\mathrm{B}\right), 2.72\left(\mathrm{~m}, 0.8 \mathrm{H} ; \mathrm{NCH}_{2}\right.$ rotamer $\left.\mathrm{B}\right), 2.50$ (br s, $0.2 \mathrm{H}$; COCH rotamer B), $2.48(\mathrm{~d}, J=3.5 \mathrm{~Hz} ; 0.8 \mathrm{H}$; $\mathrm{COCH}$ rotamer A), $2.12(\mathrm{dd}, J=12.3,4.6$ $\left.\mathrm{Hz}, 1 \mathrm{H} ; \mathrm{CH}_{2}\right), 1.89-1.64\left(\mathrm{~m}, 4 \mathrm{H} ; \mathrm{CH}_{2}\right), 1.50\left(\mathrm{~m}, 1 \mathrm{H} ; \mathrm{CH}_{2}\right), 0.81$ (t, J=7.3 Hz, 3H; $\left.\mathrm{CH}_{3}\right)$.

${ }^{13} \mathrm{C} \mathrm{NMR}\left(\mathrm{CDCl}_{3}, 100 \mathrm{MHz}\right)$ (rotamers!) $\delta$ 188.8, 156.2, 145.9, 136.6, 134.7, 132.9, 132.7, 131.0, 128.4, 128.2, 128.0, 127.8, 127.6, 127.2, 67.2, 66.8, 45.9, 45.7, 41.7, 41.2, 38.1, 37.3, $36.6,26.3,26.1,25.0,21.9,10.7$

IR v (cm-1): $2960(\mathrm{w}), 2936(\mathrm{w}), 2875$ (w), 1706 (s), 1666 (s), 1665 (s), 1460 (m), 1416 (m), 1356 (w), 1311 (w), 1310 (w), 1267 (w), 1237 (w), 1236 (w), 1202 (w), 1201 (w), 1127 (w), $1107(w), 1106(w), 1080(w), 1079$ (w), 1069 (w), 1068 (w), 1062 (w), $1036($ w), $1035(w)$, $1019(\mathrm{w}), 752(\mathrm{~m}), 740(\mathrm{~s}), 731(\mathrm{~s}), 709(\mathrm{~m})$.

HRMS(ESI) calcd for $\mathrm{C}_{21} \mathrm{H}_{24} \mathrm{NO}_{3} \mathrm{~S}^{+}(\mathrm{M}+\mathrm{H}) 370.1471$, found 370.1408 .

\section{Benzyl- $N$-benzyl- $N$-vinylcarbamate (46)}

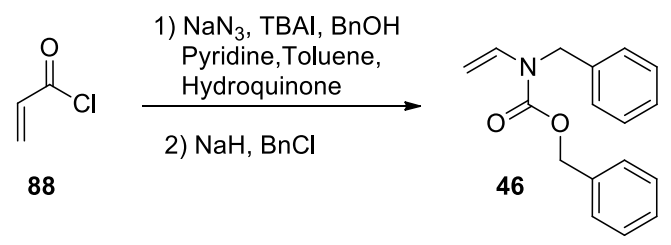

Following a slight modification of a reported procedure, ${ }^{[28,29]}$ a solution of acryloyl chloride $\mathbf{8 8}$ ( $8.97 \mathrm{~mL}, 110 \mathrm{mmol}, 1.00$ equiv) and TBAI (2.04 g, $5.52 \mathrm{mmol}, 0.05$ equiv) in toluene (100 mL) was added dropwise to a solution of sodium azide ( $8.60 \mathrm{~g}, 132 \mathrm{mmol}, 1.20$ equiv) in $\mathrm{H}_{2} \mathrm{O}$ (100 $\mathrm{mL}$ ) at $0^{\circ} \mathrm{C}$. The biphasic reaction was stirred at $0^{\circ} \mathrm{C}$ for 5 hours then the layers were separated. The organic layer was dried over $\mathrm{Na}_{2} \mathrm{SO}_{4}$ and filtered. The filtrate was added carefully into a solution of benzyl alcohol (120 g, $1.10 \mathrm{~mol}, 10$ equiv), pyridine (523 mg, $6.63 \mathrm{mmol}, 0.06$ equiv) and hydroquinone $\left(607 \mathrm{mg}, 5.52 \mathrm{mmol}, 0.05\right.$ equiv) at $85^{\circ} \mathrm{C}$. The reaction was stirred for 1 hour at $85^{\circ} \mathrm{C}$ then distilled under reduced pressure. The crude distillate $\left(140^{\circ} \mathrm{C}, 0.13 \mathrm{mmHg}\right)$ was recrystallized in cyclohexane to give benzyl-vinylcarbamate as colorless crystals $(4.82 \mathrm{~g}, 27.2$ mmol, $25 \%)$.

$R_{f} 0.3$ (AcOEt/PET 1:9 Anisaldehyde).

[28] Wieber, G. M.; Hegedus, L. S.; Akermark, B.; Michalson, E. T., J. Org. Chem. 1989, 54, 4649.

[29] Kamatani, A.; Overman, L. E., J. Org. Chem. 1999, 64 (23), 8743. 
${ }^{1} \mathrm{H}$ NMR $\left(\mathrm{CDCl}_{3}, 400 \mathrm{MHz}\right) \delta 7.33$ (m, 5H; ArH), 6.70 (m, 1H; CHN), 6.41 (br s, 1H; NH), 5.15 (s, 2H; $\left.\mathrm{CH}_{2} \mathrm{Ar}\right), 4.48$ (d, $J=15.7 \mathrm{~Hz}, 1 \mathrm{H}$; vinylH, cis), 4.30 (d, $J=8.4 \mathrm{~Hz}, 1 \mathrm{H}$, vinylH, trans).

A solution of benzyl-vinylcarbamate $(800 \mathrm{mg}, 4.52 \mathrm{mmol}, 1.00$ equiv) in THF (10 mL) was added dropwise into a suspension of $\mathrm{NaH}(163 \mathrm{mg}, 6.80 \mathrm{mmol}, 1.5$ equiv) in THF (40 mL) at $0^{\circ} \mathrm{C}$. The mixture was stirred at $0^{\circ} \mathrm{C}$ for 1.5 hour then benzyl bromide was added dropwise. The reaction was allowed to warm to $\mathrm{rt}$ and stirred overnight before quenching with aqueous $\mathrm{NaHCO}_{3}$ at $0^{\circ} \mathrm{C}$. The suspension was extracted with $\mathrm{Et}_{2} \mathrm{O}\left(30 \mathrm{~mL}\right.$ x 3), dried on $\mathrm{Na}_{2} \mathrm{SO}_{4}$, filtered and concentrated under reduced pressure. The crude product was purified by flash column chromatography (pentane/AcOEt 30:1-9:1) to give the protected enamide 46 (850 $\mathrm{mg}, 3.18$ mmol, $70 \%$ ) as a pale yellow oil.

$R_{f} 0.7$ (AcOEt/PET 1:9 Anisaldehyde).

${ }^{1} \mathrm{H}$ NMR $\left(\mathrm{CDCl}_{3}, 400 \mathrm{MHz}\right) \delta$ 7.46-7.07 (m, 11H; ArH and CHN), 5.23 (m, 2H; $\left.\mathrm{OCH}_{2} \mathrm{Ar}\right), 4.80$ (m, 2H; $\left.\mathrm{CH}_{2} \mathrm{Ar}\right), 4.44-4.18$ (m, 2H; vinylH).

\section{2-(Benzyl((benzyloxy)carbonyl)amino)cyclopropanecarboxylic acid (48)}

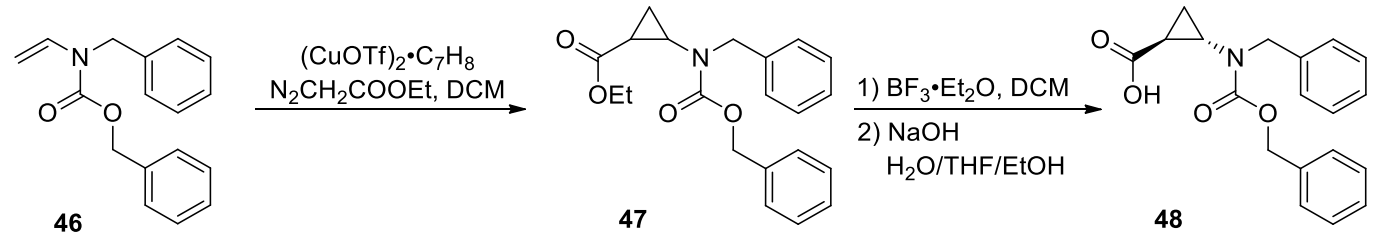

Following the reported procedure, ${ }^{[30]}$ a solution of ethyl diazoacetate $(1.5 \mathrm{~mL}, 15 \mathrm{mmol}, 4.6$ equiv) in DCM $(8 \mathrm{~mL})$ was added via a syringe pump $(0.5 \mathrm{~mL} / \mathrm{h})$ into a suspension of vinyl amide 46 (0.85 g, $3.2 \mathrm{mmol}, 1.0$ equiv) and (CuOTf) ${ }_{2} \cdot \mathrm{Tol}$ (47 mg, $92 \mu \mathrm{mol}, 0.030$ equiv) in DCM $(8 \mathrm{~mL})$. The suspension was then filtered and purified by column chromatography (pentane/AcOEt 9:1) to afford the cyclopropane derivative $47(0.78 \mathrm{~g}, 2.2 \mathrm{mmol}, 69 \%)$ as a diastereomeric mixture.

Following a reported procedure ${ }^{[31]}$ the diastereomeric mixture of esters $47(0.49 \mathrm{~g}, 1.4 \mathrm{mmol}$, 1.0 equiv) in dichlorometane $(9 \mathrm{~mL})$ at $-20^{\circ} \mathrm{C}$ was treated with $\mathrm{BF}_{3} \cdot \mathrm{Et}_{2} \mathrm{O}(40 \mathrm{mg}, 0.38 \mathrm{mmol}$, 0.20 equiv). The reaction was allowed to warm at $0^{\circ} \mathrm{C}$ and stirred at the same temperature until the isomerization was finished (from $R_{f}$ 0.2-0.3 PET/AcOEt 8:2 to $R_{f} 0.3$ PET/AcOEt 8:2, 
Anisaldehyde). Triethylamine $(1 \mathrm{~mL})$ was added dropwise to the reaction and the mixture was diluted with $\mathrm{Et}_{2} \mathrm{O}(20 \mathrm{~mL})$ and washed with water and brine. The organic layer was dried over $\mathrm{Na}_{2} \mathrm{SO}_{4}$ and evaporated under reduced pressure to give a yellow oil $(0.49 \mathrm{~g})$ which was used directly in the next step.

The crude oil ( $0.49 \mathrm{~g}, 1.4 \mathrm{mmol}, 1.0$ equiv) was dissolved in a solution of water/THF/EtOH 1/1/3 (7.5 mL total) at $0^{\circ} \mathrm{C}$ and $\mathrm{NaOH}(0.56 \mathrm{~g}, 14 \mathrm{mmol}, 10$ equiv) was added portionswise. The reaction was stirred at $0^{\circ} \mathrm{C}$ during one hour then concentrated, diluted with water $(20 \mathrm{~mL})$ and washed with $\mathrm{Et}_{2} \mathrm{O}(3 \times 20 \mathrm{~mL})$. The aqueous layer was acidified with $\mathrm{HCl}$ (1 M aqueous solution) until $\mathrm{pH} 2$ and extracted with DCM $(3 \times 20 \mathrm{~mL})$ to give $\mathbf{4 8}$ as a colorless oil which turns solid upon storage (370 mg, $1.14 \mathrm{mmol}, 82 \%$ overall). No further purification was needed.

$R_{f} 0.0$ (AcOEt/PET 3:7 Anisaldehyde).

${ }^{1} \mathrm{H}$ NMR $\left(\mathrm{CDCl}_{3}, 400 \mathrm{MHz}\right) \delta$ 7.41-7.17 (m, 10H, ArH), $5.22\left(\mathrm{~d}, J=12.4 \mathrm{~Hz}, 1 \mathrm{H} ; \mathrm{OCH}_{2} \mathrm{Ar}\right)$, $5.16\left(\mathrm{~d}, J=12.3 \mathrm{~Hz}, 1 \mathrm{H} ; \mathrm{OCH}_{2} \mathrm{Ar}\right), 4.57\left(\mathrm{~d}, J=15.4 \mathrm{~Hz}, 1 \mathrm{H} ; \mathrm{CH}_{2} \mathrm{Ar}\right), 4.42(\mathrm{~d}, J=15.4 \mathrm{~Hz}, 1 \mathrm{H}$; $\left.\mathrm{CH}_{2} \mathrm{Ar}\right), 3.06$ (m, 1H; CHNH), 1.87 (m, 1H; CHCO), $1.44\left(\mathrm{~m}, 1 \mathrm{H} ; \mathrm{CH}_{2}\right), 1.35\left(\mathrm{~m}, 1 \mathrm{H} ; \mathrm{CH}_{2}\right)$.

${ }^{13} \mathrm{C} \mathrm{NMR}\left(\mathrm{CDCl}_{3}, 100 \mathrm{MHz}\right) \delta 177.6,157.7,137.5,137.4,136.2,128.7,128.5,128.5,128.4$, $128.1,128.0,127.5,127.3,67.7,51.4,38.3,23.4,17.1 .^{[32]}$

IR v (cm-1): 3065 (w), 3035 (w), 2996 (w), 2948 (w), 1700 (s), 1700 (s), 1699 (s), 1699 (s), 1587 (w), 1543 (w), 1498 (w), 1453 (m), 1410 (s), 1410 (s), 1409 (s), 1409 (s), 1356 (m), 1325 (w), 1291 (m), 1290 (m), 1273 (m), 1238 (m), 1237 (m), 1218 (m), 1189 (m), 1178 (m), 1134 (m), 1033 (w), 980 (w), 912 (w), 911 (w), 774 (w), 737 (m), 737 (m), 737 (m), 700 (m), 700 (m), $639(\mathrm{w}), 630(\mathrm{~m}), 630(\mathrm{~m}), 630(\mathrm{~m}), 621(\mathrm{~s}), 621(\mathrm{~s}), 621(\mathrm{~s})$.

HRMS(ESI) calcd for $\mathrm{C}_{19} \mathrm{H}_{20} \mathrm{NO}_{4}{ }^{+}(\mathrm{M}+\mathrm{H}) 326.1392$, found 326.1389 .

\section{Benzyl benzyl(2-(1-methyl-1H-indole-2-carbonyl)cyclopropyl)carbamate (21)}
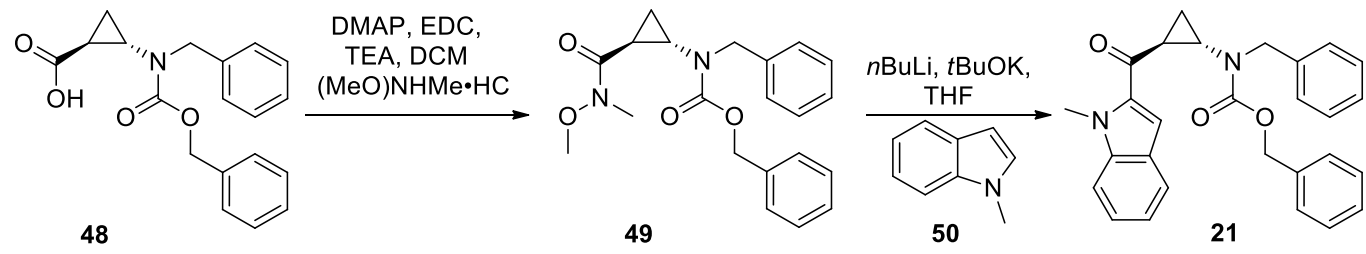

[32] Not all the aromatic signals were resolved 
Dimethylhydroxylamine hydrochloride ( $55 \mathrm{mg}, 0.57 \mathrm{mmol}, 1.5$ equiv) was added to a solution of acid 48 (0.12 g, $0.38 \mathrm{mmol}, 1.0$ equiv) in DCM (3 mL) at rt. The reaction was stirred until complete dissolution then DMAP ( $9 \mathrm{mg}, 7 \mu \mathrm{mol}, 0.2$ equiv) and EDC ( $0.11 \mathrm{~g}, 0.57 \mathrm{mmol}, 1.5$ equiv) were added portionwise followed by the dropwise addition of TEA ( $79 \mu \mathrm{L}, 0.57 \mathrm{mmol}$, 1.5 equiv). The solution was stirred overnight then quenched with a $1 \mathrm{M}$ solution of $\mathrm{HCl}(2 \mathrm{~mL})$, diluted in DCM $(20 \mathrm{~mL})$, washed with a $1 \mathrm{M}$ solution of $\mathrm{HCl}(3 \times 4 \mathrm{~mL})$ and a saturated aqueous solution of $\mathrm{NaHCO}_{3}(2 \times 4 \mathrm{~mL})$, dried over $\mathrm{MgSO}_{4}$ and concentrated under reduced pressure. The crude product was purified by flash column chromatography $\left(\mathrm{Et}_{2} \mathrm{O} /\right.$ pentane $\left.7: 3\right)$ to yield the Weinreb amide $49\left(0.22 \mathrm{~g}, 0.33 \mathrm{mmol}, 86 \%, \mathrm{R}_{f}=0.5\left(\mathrm{Et}_{2} \mathrm{O} /\right.\right.$ pentane 8:2) as a colorless oil, which was used directly in the next step.

$N$-Methyl indole 50 (0.15 g, $1.1 \mathrm{mmol}, 1.3$ equiv) was diluted in THF (7 mL) at $0{ }^{\circ} \mathrm{C}$ and ${ }^{n} \mathrm{BuLi}$ ( $0.42 \mathrm{~mL}, 1.0 \mathrm{mmol}, 1.2$ equiv) was added dropwise. The reaction mixture was stirred $1 \mathrm{~h}$ at $\mathrm{rt}$ and cooled to $-78{ }^{\circ} \mathrm{C}$. Weinreb amide 49 (dried through 3 co-evaporation with toluene, $0.32 \mathrm{~g}$, $0.87 \mathrm{mmol}, 1.0$ equiv) was added dropwise via cannula as a cooled $\left(-78^{\circ} \mathrm{C}\right)$ solution in THF $(7$ $\mathrm{mL})$. After further stirring $2 \mathrm{~h}$ at $-78{ }^{\circ} \mathrm{C}$, a solution of $\mathrm{KO} t \mathrm{Bu}(0.11 \mathrm{~g}, 1.7 \mathrm{mmol}, 2.0$ equiv) in THF ( $1 \mathrm{~mL})$ was added. After $2 \mathrm{~h}$, the reaction mixture was left to warm up to $-20{ }^{\circ} \mathrm{C}$ and stirred. After 20 min at $-20{ }^{\circ} \mathrm{C}$, the reaction mixture was transferred via cannula into a saturated aqueous solution of $\mathrm{NaHCO}_{3}(20 \mathrm{~mL})$ at $0^{\circ} \mathrm{C}$ and stirred for 20 minutes before extraction with $\mathrm{Et}_{2} \mathrm{O}(3 \times 20$ $\mathrm{mL})$. The combined organic layers were washed with brine $(10 \mathrm{~mL})$, dried over $\mathrm{MgSO}_{4}$ and the solvent was removed under reduced pressure. The crude product was purified by flash column chromatography (PET/AcOEt 9:1-8:2) to yield indole 21 (243 mg, $0.554 \mathrm{mmol}, 64 \%$ ) as an orange oil.

$R_{f}$ 0.6 (AcOEt/PET 3:7 Anisaldehyde).

${ }^{1} \mathrm{H}$ NMR $\left(\mathrm{CDCl}_{3}, 400 \mathrm{MHz}\right) \delta 7.70(\mathrm{~d}, J=8.0 \mathrm{~Hz}, 1 \mathrm{H} ; \mathrm{ArH}), 7.47-7.06(\mathrm{~m}, 14 \mathrm{H} ; \mathrm{ArH}), 5.20(\mathrm{~d}$, $\left.J=12.2 \mathrm{~Hz}, 1 \mathrm{H} ; \mathrm{OCH}_{2} \mathrm{Ar}\right), 5.13\left(\mathrm{~d}, J=12.2 \mathrm{~Hz}, 1 \mathrm{H} ; \mathrm{OCH}_{2} \mathrm{Ar}\right), 4.76(\mathrm{~d}, J=15.3 \mathrm{~Hz}, 1 \mathrm{H}$; $\mathrm{CH}_{2} \mathrm{Ar}$ ), 4.45 (d, $J=15.4 \mathrm{~Hz}, 1 \mathrm{H} ; \mathrm{CH}_{2} \mathrm{Ar}$ ), 3.99 (s, $3 \mathrm{H}, \mathrm{NCH}_{3}$ ), 3.20 (m, 1H; CHNH), 2.93 (m, 1H, CHCO), 1.67 (d, J=5.4 Hz, 1H; $\left.\mathrm{CH}_{2}\right), 1.45\left(\mathrm{~m}, 1 \mathrm{H} ; \mathrm{CH}_{2}\right)$.

${ }^{13} \mathrm{C} \mathrm{NMR}\left(\mathrm{CDCl}_{3}, 100 \mathrm{MHz}\right) \delta 190.3,157.2,140.1,137.8,136.0,135.1,128.6,128.2,128.0$, $127.8,127.5,127.4,125.9,125.9,122.9,120.6,112.0,110.3,67.6,51.5,40.4,32.0,29.3$, 18.0.

IR v (cm-1): 3059 (w), $3031(w), 3031(w), 2949$ (w), 1706 (s), 1649 (s), 1615 (w), 1514 (m), 1466 (m), 1456 (m), 1430 (m), 1404 (s), 1385 (m), 1353 (m), 1321 (m), 1229 (m), 1228 (m), 
1200 (m), 1166 (m), 1152 (m), 1129 (m), 1041 (w), 1005 (m), 936 (w), 771 (m), 744 (s), 700 (s), $640(\mathrm{~m}), 631(\mathrm{~m}), 605(\mathrm{~m})$.

HRMS(ESI) calcd for $\mathrm{C}_{28} \mathrm{H}_{27} \mathrm{~N}_{2} \mathrm{O}_{3}{ }^{+}(\mathrm{M}+\mathrm{H}) 439.2022$, found 439.2033.

\section{Benzyl (2-(1H-indole-2-carbonyl)cyclopropyl)(benzyl)carbamate (22)}
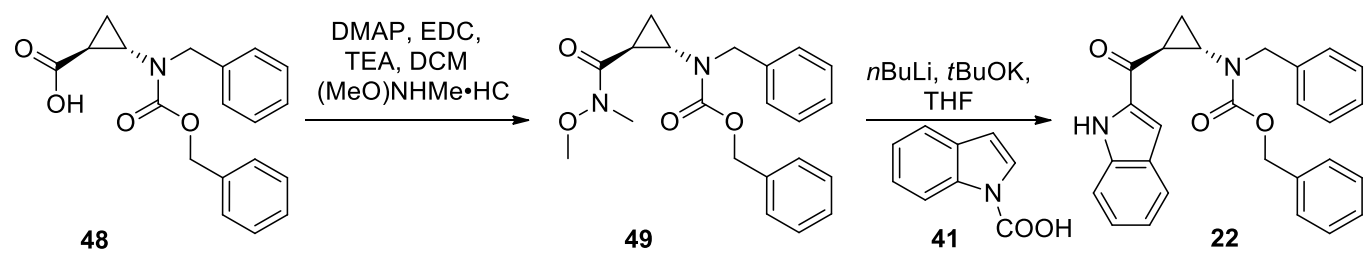

Dimethylhydroxylamine hydrochloride ( $55 \mathrm{mg}, 0.57 \mathrm{mmol}, 1.5$ equiv) was added to a solution of acid 48 (0.12 g, $0.38 \mathrm{mmol}, 1.0$ equiv) in DCM (3 mL) at rt. The reaction was stirred until complete dissolution then DMAP ( $9 \mathrm{mg}, 7 \mu \mathrm{mol}, 0.2$ equiv) and EDC $(0.11 \mathrm{~g}, 0.57 \mathrm{mmol}, 1.5$ equiv) were added portionwise followed by the dropwise addition of TEA ( $79 \mu \mathrm{L}, 0.57 \mathrm{mmol}$, 1.5 equiv). The solution was stirred overnight then quenched with a $1 \mathrm{M}$ solution of $\mathrm{HCl}(2 \mathrm{~mL})$, diluted in DCM (20 mL), washed with a $1 \mathrm{M}$ solution of $\mathrm{HCl}(3 \times 4 \mathrm{~mL})$ and a saturated aqueous solution of $\mathrm{NaHCO}_{3}(2 \times 4 \mathrm{~mL})$, dried over $\mathrm{MgSO}_{4}$ and concentrated under reduced pressure. The crude product was purified by flash column chromatography $\left(\mathrm{Et}_{2} \mathrm{O} /\right.$ pentane $\left.7: 3\right)$ to yield Weinreb amide $49\left(0.22 \mathrm{~g}, 0.33 \mathrm{mmol}, 86 \%, \mathrm{R}_{\mathrm{f}}=0.5\left(\mathrm{Et}_{2} \mathrm{O} /\right.\right.$ pentane 8:2) as a colorless oil, which was used directly in the next step.

$t \operatorname{BuLi}(0.74 \mathrm{~mL}, 1.2 \mathrm{mmol}, 2.2$ equiv) was added dropwise into a solution of $N$-carboxylindole prepared following GP6 (96 mg, $0.59 \mathrm{mmol}, 1.1$ equiv) and $\mathrm{LiCl}$ (25 mg, $0.59 \mathrm{mmol}, 1.1$ equiv) in THF $(1.5 \mathrm{~mL})$ at $-78^{\circ} \mathrm{C}$. The reaction mixture was stirred $3 \mathrm{~h}$ at $-78^{\circ} \mathrm{C}$ then a solution of Weinreb amide 49 (0.20 g, $0.54 \mathrm{mmol}, 1.0$ equiv) was added dropwise via cannula as a cooled ($\left.78{ }^{\circ} \mathrm{C}\right)$ solution in THF $(1.5 \mathrm{~mL})$. The reaction mixture was left to warm up to $-20{ }^{\circ} \mathrm{C}$ and stirred. After 20 min at $-20{ }^{\circ} \mathrm{C}$, the reaction was transferred via cannula into a saturated aqueous solution of $\mathrm{NaHCO}_{3}(10 \mathrm{~mL})$ at $0^{\circ} \mathrm{C}$ and stirred for 20 minutes before the extraction with $\mathrm{Et}_{2} \mathrm{O}(3 \times 10$ $\mathrm{mL})$. The combined organic layers were washed with brine $(10 \mathrm{~mL})$, dried over $\mathrm{MgSO}_{4}$ and the solvent was removed under reduced pressure. The crude product was purified by flash column chromatography (PET/AcOEt 9:1-8:2) to yield indole 22 (82 $\mathrm{mg}, 0.19 \mathrm{mmol}, 36 \%$ ) as a yellow oil. 
$R_{f} 0.7$ (AcOEt/PET 3:7 Anisaldehyde).

${ }^{1} \mathrm{H}$ NMR $\left(\mathrm{CDCl}_{3}, 400 \mathrm{MHz}\right) \delta 9.78(\mathrm{br} \mathrm{s}, 1 \mathrm{H} ; \mathrm{NH}), 7.71(\mathrm{~d}, J=8.1 \mathrm{~Hz}, 1 \mathrm{H} ; \mathrm{ArH}), 7.45-7.06(\mathrm{~m}$, $14 \mathrm{H} ; \mathrm{ArH}$ ), 5.24 (d, $J=12.2 \mathrm{~Hz}, 1 \mathrm{H} ; \mathrm{OCH}_{2} \mathrm{Ar}$ ), 5.14 (br m, $1 \mathrm{H} ; \mathrm{OCH}_{2} \mathrm{Ar}$ ), 4.78 (d, $J=15.2 \mathrm{~Hz}$, $\left.1 \mathrm{H} ; \mathrm{CH}_{2} \mathrm{Ar}\right), 4.45$ (d, $\left.J=15.4 \mathrm{~Hz}, 1 \mathrm{H} ; \mathrm{CH}_{2} \mathrm{Ar}\right), 3.28(\mathrm{~m}, 1 \mathrm{H} ; \mathrm{CHN}), 2.88(\mathrm{~m}, 1 \mathrm{H} ; \mathrm{CHCO}), 1.76$ (m, $\left.1 \mathrm{H} ; \mathrm{CH}_{2}\right), 1.50\left(\mathrm{~m}, 1 \mathrm{H} ; \mathrm{CH}_{2}\right)$.

${ }^{13} \mathrm{C} \mathrm{NMR}\left(\mathrm{CDCl}_{3}, 100 \mathrm{MHz}\right)$ (rotamers!) $\delta 189.6,157.1,137.6,137.5,136.0,135.3,128.6$, $128.4,128.2,127.9,127.9,127.5,127.4,126.9,126.5,126.2,124.1,123.0,121.7,120.7,120.6$, 119.6, 112.2, 111.0, 110.0, 102.3, 67.6, 65.1, 51.5, 40.5, 28.1, 26.1, 18.2.

IR v (cm-1): $3308(\mathrm{w}), 3307(\mathrm{w}), 3281(\mathrm{w}), 3063(\mathrm{w}), 3032(\mathrm{w}), 2957(\mathrm{w}), 1699(\mathrm{~s}), 1636(\mathrm{~m})$, 1574 (w), 1523 (m), 1497 (m), 1455 (m), 1436 (m), 1400 (s), 1353 (m), 1344 (m), 1299 (m), 1298 (m), 1265 (m), 1229 (m), 1204 (m), 1165 (m), 1140 (s), 1079 (m), 1068 (m), 1049 (w), 1048 (w), 1029 (m), 982 (w), 982 (w), 960 (w), 910 (m), 805 (m), 771 (m), 736 (s), 736 (s), 699 (s), $619(\mathrm{~m})$.

HRMS(ESI) calcd for $\mathrm{C}_{27} \mathrm{H}_{25} \mathrm{~N}_{2} \mathrm{O}_{3}{ }^{+}(\mathrm{M}+\mathrm{H}) 425.1860$, found 425.1852.

\section{7-Carbethoxy-2-oxabicyclo[4.1.0]heptane (51)}

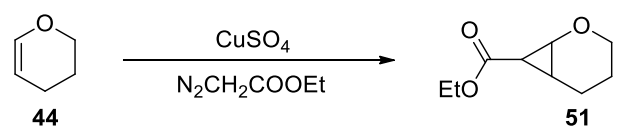

Following a reported procedure, ${ }^{[33]}$ a solution of ethyl diazoacetate $(5.0 \mathrm{~mL}, 48 \mathrm{mmol}, 1.0$ equiv) in freshly distilled dihydropyran $44(9.0 \mathrm{~mL})$ was added via a syringe pump to a refluxing solution of $\mathrm{CuSO}_{4}$ (0.38 mg, $2.4 \mathrm{mmol}, 0.050$ equiv) in dihydropyran 44 (21 mL) over 2.5 hours. The reaction was refluxed for additional $2 \mathrm{~h}$. The excess of dihydropyran was removed under atmospheric pressure and the resulting solution was distilled $\left(90{ }^{\circ} \mathrm{C}, 3 \mathrm{mmHg}\right)$ to give cyclopropane $\mathbf{5 1}$ (5.0 g, $29 \mathrm{mmol}, 62 \%)$ as a colorless oil.

$R_{f} 0.8$ (AcOEt/PET 2:8 Anisaldehyde).

${ }^{1} \mathrm{H} \mathrm{NMR}\left(\mathrm{CDCl}_{3}, 400 \mathrm{MHz}\right) \delta 4.12\left(\mathrm{~m}, 2 \mathrm{H} ; \mathrm{COOCH}_{2}\right), 3.91(\mathrm{~m}, 1 \mathrm{H} ; \mathrm{OCH}), 3.60(\mathrm{dtd}, J=10.8$, $\left.3.3,1.0 \mathrm{~Hz}, 1 \mathrm{H} ; \mathrm{OCH}_{2}\right), 3.36\left(\mathrm{td}, J=11.3,2.2 \mathrm{~Hz}, 1 \mathrm{H} ; \mathrm{OCH}_{2}\right), 2.11-1.83(\mathrm{~m}, 3 \mathrm{H} ; \mathrm{CHCO}, \mathrm{CH}$

[33] Temme, O.; Taj, S. A.; Andersson, P. G., J. Org. Chem. 1998, 63, 6007. 
and $\left.\mathrm{CH}_{2}\right), 1.75\left(\mathrm{~m}, 1 \mathrm{H} ; \mathrm{CH}\right.$ or $\left.\mathrm{CH}_{2}\right), 1.60-1.40\left(\mathrm{~m}, 2 \mathrm{H} ; \mathrm{CH}\right.$ or $\left.\mathrm{CH}_{2}\right), 1.26(\mathrm{t}, 3 \mathrm{H}, J=7.2 \mathrm{~Hz}$, $\left.\mathrm{CH}_{3}\right)$.

\section{2-Oxabicyclo[4.1.0]heptan-7-yl(1H-indol-2-yl)methanone (23)}

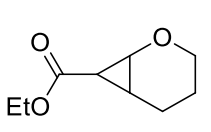

51

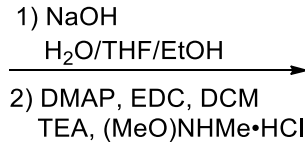

TEA, (MeO)NHMe•HC

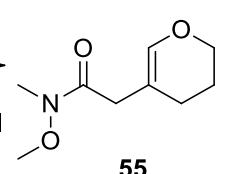

55

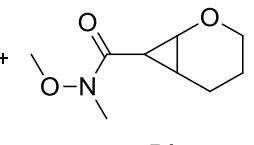

54

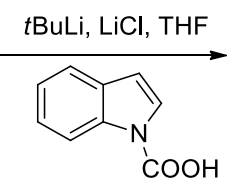

41

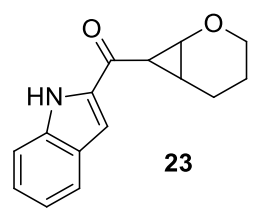

The ester 51 (1.50 g, $8.82 \mathrm{mmol}, 1.00$ equiv) was dissolved in a solution of water/THF/EtOH $1 / 1 / 3(25 \mathrm{~mL}$ total $)$ at $0^{\circ} \mathrm{C}$ and $\mathrm{NaOH}(2.47 \mathrm{~g}, 61.8 \mathrm{mmol}, 7.00$ equiv) was added portionswise. The reaction was stirred during 20 minutes at $0^{\circ} \mathrm{C}$ then diluted with water $(30 \mathrm{~mL})$ and washed with $\mathrm{Et}_{2} \mathrm{O}(3 \times 20 \mathrm{~mL})$. The aqueous layer was acidified with $\mathrm{HCl}(1 \mathrm{M}$ aqueous solution) until $\mathrm{pH}$ 2 and extracted with DCM $(3 \times 20 \mathrm{~mL})$ to give the carboxylic acid $\mathbf{5 2}(1.16 \mathrm{~g}, 8.17 \mathrm{mmol}, 93 \%$, 7:1 mixture of open and closed form) as a white solid which was used directly in the next step.

Dimethylhydroxylamine hydrochloride $(0.54 \mathrm{~g}, 5.6 \mathrm{mmol}, 1.5$ equiv $)$ was added to a solution of acid 52 (0.53 g, $3.7 \mathrm{mmol}, 1.0$ equiv) in DCM $(30 \mathrm{~mL})$ at rt. The reaction was stirred until complete dissolution, then DMAP (91 mg, $0.75 \mathrm{mmol}, 0.2$ equiv) and EDC (1.0 g, $5.6 \mathrm{mmol}, 1.5$ equiv) were added portionwise followed by the dropwise addition of TEA (0.74 mL, $5.6 \mathrm{mmol}$, 1.5 equiv). The solution was stirred overnight then quenched with a $1 \mathrm{M}$ solution of $\mathrm{HCl}(2 \mathrm{~mL})$, diluted in DCM (20 mL), dried over $\mathrm{MgSO}_{4}$ and concentrated under reduced pressure. The crude product was purified by flash column chromatography (AcOEt/PET 3:7) to yield Weinreb amide $54\left(0.41 \mathrm{~g}, 0.22 \mathrm{mmol}, 59 \%, \mathrm{R}_{\mathrm{f}}=0.3(\right.$ AcOEt/PET 3:7) as an inseparable mixture of open and closed form in 2:1 ratio respectively.

$t$ BuLi (1.6 M in pentane, $3.6 \mathrm{~mL}, 5.8 \mathrm{mmol}, 2.6$ equiv) was added dropwise into a solution of $N$ carboxylindole prepared following GP6 (0.46 g, $2.9 \mathrm{mmol}, 1.3$ equiv) and $\mathrm{LiCl}(0.24 \mathrm{~g}, 5.8$ mmol, 2.6 equiv) in THF $(20 \mathrm{~mL})$ at $-78^{\circ} \mathrm{C}$. The lithiated indole was then added via cannula into the solution of Weinreb amide 54 (as a mixture of open and closed form in 2:1 ratio respectively) ( $0.41 \mathrm{~g}, 2.2 \mathrm{mmol}, 1.0$ equiv) in THF $(20 \mathrm{~mL})$ at $0^{\circ} \mathrm{C}$ during 20 minutes. The mixture was stirred for additional 15 minutes, transferred via cannula into a saturated aqueous solution of $\mathrm{NaHCO}_{3}$ $(20 \mathrm{~mL})$ at $0^{\circ} \mathrm{C}$ and extracted with $\mathrm{Et}_{2} \mathrm{O}(3 \times 20 \mathrm{~mL})$. The organic layers were dried over $\mathrm{Na}_{2} \mathrm{SO}_{4}$, evaporated under reduced pressure and purified on flash chromatography (AcOEt/PET 2:8 on deactivated silica) to give $23(0.22 \mathrm{~g}, 0.90 \mathrm{mmol}, 41 \%)$ (60\% calculated on purity of starting material) as a yellow oil. 
$R_{f} 0.4$ (AcOEt/PET 2:8 Anisaldehyde).

${ }^{1} \mathrm{H} \mathrm{NMR}\left(\mathrm{CDCl}_{3}, 400 \mathrm{MHz}\right) \delta 9.18($ br s, $1 \mathrm{H} ; \mathrm{NH}), 7.73(\mathrm{~d}, J=7.8 \mathrm{~Hz}, 1 \mathrm{H} ; \mathrm{ArH}), 7.42(\mathrm{~d}, J=$ $8.3 \mathrm{~Hz}, 1 \mathrm{H} ; \mathrm{ArH}), 7.34$ (m, 2H; ArH), 7.16 (t, $J=7.7 \mathrm{~Hz}, 1 \mathrm{H} ; \mathrm{ArH}) 4.13$ (dd, $J=7.2,1.4 \mathrm{~Hz}$, 1H; OCH), 3.72 (dt, $\left.J=10.8,2.8 \mathrm{~Hz}, 1 \mathrm{H} ; \mathrm{OCH}_{2}\right), 3.47$ (m, 1H; $\left.\mathrm{OCH}_{2}\right), 2.79(\mathrm{dd}, J=5.8,1.4 \mathrm{~Hz}$, 1H; CHCO), 2.17 (m, 1H; $\mathrm{CH}$ or $\mathrm{CH}_{2}$ ), 2.05 (m, 2H; $\mathrm{CH}$ or $\left.\mathrm{CH}_{2}\right), 1.66$ (m, 2H; $\mathrm{CH}$ or $\mathrm{CH}_{2}$ ).

${ }^{13} \mathrm{C} \mathrm{NMR}\left(\mathrm{CDCl}_{3}, 100 \mathrm{MHz}\right) \delta 189.7,137.1,135.7,127.7,126.1,123.0,120.8,112.1,109.1$, $64.8,63.6,32.4,25.2,22.0,19.2$.

IR v (cm-1): 3307 (w), 2952 (w), 2865 (w), 1629 (m), 1523 (m), 1424 (w), 1343 (w), 1276 (w), 1232 (w), 1207 (w), 1177 (w), 1132 (m), 1065 (m), 958 (w), 925 (w), $896(\mathrm{w}), 874(\mathrm{w}), 850(\mathrm{w})$, $810(\mathrm{~m}), 743(\mathrm{~s}), 667(\mathrm{~s})$.

HRMS(ESI) calcd for $\mathrm{C}_{15} \mathrm{H}_{16} \mathrm{NO}_{2}^{+}(\mathrm{M}+\mathrm{H})$ 242.1181, found 242.1187.

\section{Cyclization of amino- and oxycyclopropanes}

Benzyl 4a-ethyl-7-methyl-6-oxo-2,3,4,4a,5,6,7,11c-octahydro-1H-pyrido[3,2-c]carbazole-1carboxylate (56) and 4a-ethyl-7-methyl-3,4,4a,5,7,11c-hexahydro-1H-pyrido[3,2c]carbazol-6(2H)-one (89)
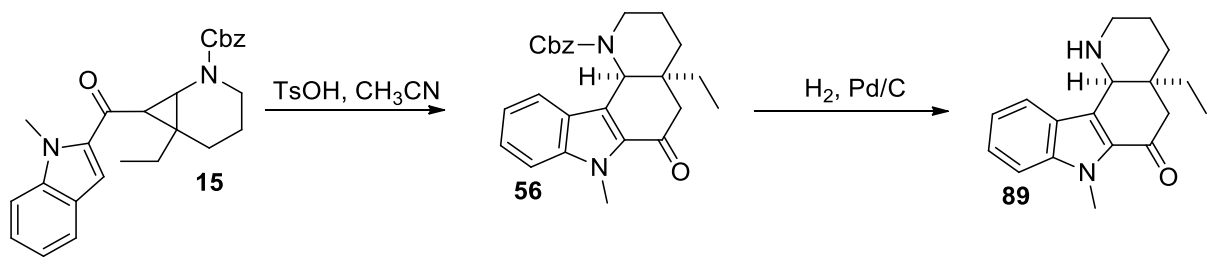

The reaction was performed following general procedure GP4, starting from cyclopropane 15 (14 mg, $0.034 \mathrm{mmol}, 1.0$ equiv) and tosic acid ( $1 \mathrm{mg}, 7 \mu \mathrm{mol}, 0.2$ equiv). The reaction was quenched after $5 \mathrm{~min}$ to give 56 (14 $\mathrm{mg}, 0.034 \mathrm{mmol}$, quant) without further purification as yellow oil ( $R_{f} 0.65$ (PET/AcOEt 7:3, Anisaldehyde)). The indole derivative 56 (14 mg, $34 \mu \mathrm{mol}$, 1.0 equiv) was deprotected following general procedure GP5, using Pd/C (3 mg 10\% w/w) in $\mathrm{Et}_{2} \mathrm{O}(1.5 \mathrm{~mL})$ and $\mathrm{H}_{2}$ balloon. The suspension was filtered on celite and washed with DCM to afford $\mathbf{8 9}$ as green oil in quantitative yield $(9.6 \mathrm{mg}, 34 \mu \mathrm{mol})$.

$R_{f} 0.75\left(\mathrm{DCM} / \mathrm{MeOH} / \mathrm{Et}_{3} \mathrm{~N}\right.$ 3:1:2\%, Anisaldehyde). 
${ }^{1} \mathrm{H}$ NMR $\left(\mathrm{CDCl}_{3}, 400 \mathrm{MHz}\right) \delta 8.01(\mathrm{~d}, J=8.2 \mathrm{~Hz}, 1 \mathrm{H} ; \mathrm{ArH}), 7.45-7.32(\mathrm{~m}, 2 \mathrm{H} ; \mathrm{ArH}), 7.22(\mathrm{t}, J$ $=7.2 \mathrm{~Hz}, 1 \mathrm{H} ; \mathrm{ArH}), 4.16(\mathrm{~s}, 1 \mathrm{H} ; \mathrm{CHN}), 4.08\left(\mathrm{~s}, 3 \mathrm{H} ; \mathrm{NCH}_{3}\right), 3.53\left(\mathrm{~d}, J=17.0 \mathrm{~Hz}, 1 \mathrm{H} ; \mathrm{CH}_{2} \mathrm{CO}\right)$, $2.52\left(\mathrm{~m}, J=14.6 \mathrm{~Hz}, 2 \mathrm{H} ; \mathrm{CH}_{2} \mathrm{~N}\right), 2.26\left(\mathrm{~d}, J=16.8 \mathrm{~Hz}, 1 \mathrm{H} ; \mathrm{CH}_{2} \mathrm{CO}\right), 1.87-1.21\left(\mathrm{~m}, 7 \mathrm{H} ; \mathrm{CH}_{2}\right.$ and $\mathrm{NH}), 0.75\left(\mathrm{t}, J=7.4 \mathrm{~Hz}, 3 \mathrm{H} ; \mathrm{CH}_{3}\right)$.

${ }^{13} \mathrm{C} \mathrm{NMR}\left(\mathrm{CDCl}_{3}, 100 \mathrm{MHz}\right) \delta 191.6,139.8,129.9,126.6,124.3,121.5,120.8,110.3,110.3$, 55.6, 45.0, 44.0, 40.2, 32.6, 31.6, 31.2, 29.7, 27.5.

IR v (cm-1): 3300 (w), 2960 (m), 2935 (m), 2902 (w), 1702 (s), 1662 (s), 1615 (w), 1601 (w), 1542 (w), 1448 (m), 1411 (m), 1314 (m), 1252 (m), 1179 (w), 1117 (w), 1070 (w), 751 (m), 698 (m), $641(\mathrm{~m}), 627(\mathrm{~m}), 607(\mathrm{~m})$.

HRMS(ESI) calcd for $\mathrm{C}_{18} \mathrm{H}_{23} \mathrm{~N}_{2} \mathrm{O}^{+}(\mathrm{M}+\mathrm{H})$ 283.1810, found 283.1813.

Benzyl 4a-ethyl-6-oxo-2,3,4,4a,5,6,7,11c-octahydro-1H-pyrido[3,2-c]carbazole-1carboxylate (57); 4a-ethyl-7-3,4,4a,5,7,11c-hexahydro- $1 \mathrm{H}$-pyrido[3,2-c]carbazol-6(2H)-one (58); benzyl 4a-ethyl-6-oxo-2,3,4,4a,5,6-hexahydroindolo[1,2-a][1,8]naphthyridine-1(12aH)carboxylate (90); 4a-ethyl-1,2,3,4,4a,5-hexahydroindolo[1,2-a][1,8]naphthyridin-6(12aH)one (91)

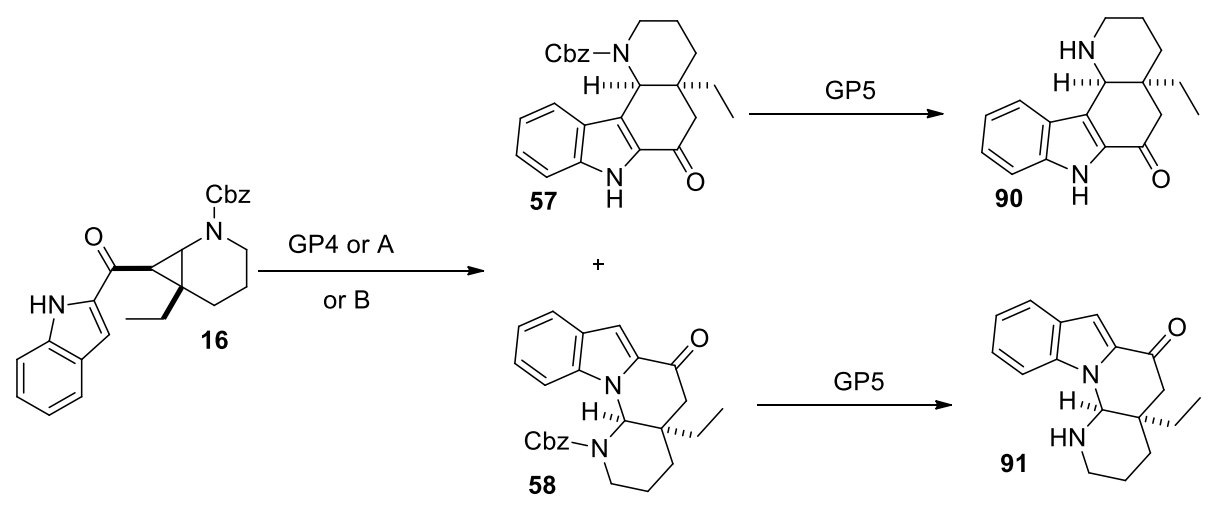

The reaction was performed following general procedure GP4, starting from cyclopropane 16 (35 mg, $0.087 \mathrm{mmol}, 1.0$ equiv) and tosic acid ( $3.0 \mathrm{mg}, 16 \mu \mathrm{mol}, 0.20$ equiv). The reaction was quenched after $15 \mathrm{~min}$ and the crude oil was purified by column chromatography (PET/AcOEt 9:1, Anisaldehyde) to give the product $57(R f 0.65,16 \mathrm{mg}, 39 \mu \mathrm{mol}, 45 \%)$ and the product $\mathbf{5 8}$ (Rf 0.75, $10 \mathrm{mg}, 25 \mu \mathrm{mol}, 29 \%$ ). The tetracyclic compound 57 (16 mg, $16 \mu \mathrm{mol}, 1.0$ equiv) was deprotected following the general procedure GP5 to give 90 (10 mg, $38 \mu \mathrm{mol}, 97 \%)$ as a yellow oil without further purification. Product 58 (10 mg, $25 \mu \mathrm{mol}, 1.0$ equiv) was deprotected 
following the general procedure GP5 to give $91(6.7 \mathrm{mg}, 25 \mu \mathrm{mol}, 99 \%)$ as a yellow oil without further purification.

\section{A: C-3 carbon cyclization}

A solution of Copper(II) triflate $\left(9.0 \mathrm{mg}, 25 \mu \mathrm{mol}, 0.10\right.$ equiv) in anhydrous $\mathrm{CH}_{3} \mathrm{CN}(250 \mu \mathrm{L})$ was added dropwise to a solution cyclopropane 16 (100 $\mathrm{mg}, 0.250 \mathrm{mmol}, 1.00$ equiv) in anhydrous $\mathrm{CH}_{3} \mathrm{CN}(12.5 \mathrm{~mL})$. The reaction was stirred during $15 \mathrm{~min}$ then quenched with $\mathrm{NaHCO}_{3}(10 \mathrm{~mL})$ and extracted with $\mathrm{Et}_{2} \mathrm{O}(3 \times 10 \mathrm{~mL})$. The combined organic layers were washed with brine, dried over $\mathrm{MgSO}_{4}$ and the solvent was removed under reduced pressure. The crude oil was purified by column chromatography (PET/AcOEt 7:3, Anisaldehyde) to give the product $57(R f 0.65,80 \mathrm{mg}, 0.20 \mathrm{mmol}, 80 \%)$ and the product $58(R f 0.75,11 \mathrm{mg}, 30 \mu \mathrm{mol}$, 11\%). The tetracyclic compound $57(70 \mathrm{mg}, 0.17 \mathrm{mmol}, 1.0$ equiv) was deprotected following the general procedure GP5 to give 90 (45 mg, $0.17 \mathrm{mmol}$, quant.) as a yellow oil without further purification.

\section{B: $\mathbf{N}-1$ nitrogen cyclization}

Toluenesulfonic acid (4.7 mg, $25 \mu \mathrm{mol}, 0.1$ equiv) was added to a solution of cyclopropane $\mathbf{1 6}$ $(0.10 \mathrm{~g}, 0.25 \mathrm{mmol}, 1.0$ equiv) in DCM $(12.5 \mathrm{~mL})$. The reaction was stirred during $15 \mathrm{~min}$ then quenched with $\mathrm{NaHCO}_{3}(10 \mathrm{~mL})$ and extracted with $\mathrm{Et}_{2} \mathrm{O}(3 \times 10 \mathrm{~mL})$. The combined organic layers were washed with brine, dried over $\mathrm{MgSO}_{4}$ and the solvent was removed under reduced pressure. The crude oil was purified by column chromatography (PET/AcOEt 7:3, Anisaldehyde) to give the product $\mathbf{5 7}(R f 0.65,4.0 \mathrm{mg}, 10 \mu \mathrm{mol}, 4 \%)$ and the product $\mathbf{5 8}(R f 0.75,85 \mathrm{mg}, 0.22$ mmol, $85 \%)$. The tetracyclic compound $58(50 \mathrm{mg}, 0.12 \mathrm{mmol}, 1.0$ equiv) was deprotected following the general procedure GP5 to give 91 (33 $\mathrm{mg}, 0.12 \mathrm{mmol}$, quant.) as a yellow oil without further purification.

\section{From 58 to 57}

Copper II triflate $(4.0 \mathrm{mg}, 11 \mu \mathrm{mol}, 0.20$ equiv) was added into a solution of $\mathbf{5 8}$ (22 $\mathrm{mg}, 55 \mu \mathrm{mol}$, 1.0 equiv) in $\mathrm{CH}_{3} \mathrm{CN}(2 \mathrm{~mL})$. The reaction was stirred at $\mathrm{rt}$ during $48 \mathrm{~h}$ then quenched with a saturated aqueous solution of $\mathrm{NaHCO}_{3}$, extracted with $\mathrm{Et}_{2} \mathrm{O}(3 \mathrm{x})$, dried on $\mathrm{Na}_{2} \mathrm{SO}_{4}$ and concentrated on reduced pressure. NMR of crude compound showed exclusively the presence of 57. 
$R_{f} 0.0$ (AcOEt/PET 6:4, Anisaldehyde).

${ }^{1} \mathrm{H} \mathrm{NMR}\left(\mathrm{CD}_{3} \mathrm{OD}, 400 \mathrm{MHz}\right) \delta 7.91(\mathrm{~d}, J=8.2 \mathrm{~Hz}, 1 \mathrm{H}$; ArH), $7.51(\mathrm{~d}, J=8.4 \mathrm{~Hz}, 1 \mathrm{H} ; \mathrm{ArH})$, 7.40 (d, $J=7.2 \mathrm{~Hz}, 1 \mathrm{H}$; ArH), 7.23 (t, $J=7.5 \mathrm{~Hz}, 1 \mathrm{H} ; \mathrm{ArH}), 4.61$ (s, 1H; CHN), 3.40-3.11 (m, $3 \mathrm{H} ; \mathrm{CH}_{2} \mathrm{~N}$ and $\mathrm{CH}_{2} \mathrm{CO}$ ), 2.37 (d, $\left.J=17.6 \mathrm{~Hz}, 1 \mathrm{H} ; \mathrm{CH}_{2} \mathrm{CO}\right), 2.16(\mathrm{~s}, 1 \mathrm{H} ; \mathrm{NH}), 1.92$ (m, 2H; $\left.\mathrm{CH}_{2}\right), 1.80$ (m, 2H; $\left.\mathrm{CH}_{2}\right), 1.45\left(\mathrm{~m}, 2 \mathrm{H} ; \mathrm{CH}_{2}\right), 0.83$ (t, J=7.5 Hz, 3H; $\left.\mathrm{CH}_{3}\right)$.

${ }^{13} \mathrm{C}$ NMR $\left(\mathrm{CD}_{3} \mathrm{OD}, 100 \mathrm{MHz}\right) \delta 191.2,140.0,132.6,128.2,126.1,122.4,122.0,121.6,114.1$, 55.6, 45.3, 42.7, 41.5, 33.0, 32.7, 20.4, 7.8.

IR v (cm-1): 2961 (s), 2943 (s), 2866 (s), 2142 (m), 1660 (w), 1543 (w), 1493 (m), 1464 (m), 1366 (w), 1263 (w), 1167 (m), 1105 (m), 1073 (m), 1021 (m), 884 (m), 804 (m), 751 (m), 673 (s), $635(\mathrm{~s})$.

HRMS(ESI) calcd for $\mathrm{C}_{17} \mathrm{H}_{21} \mathrm{~N}_{2} \mathrm{O}^{+}(\mathrm{M}+\mathrm{H}) 269.1654$, found 269.1655.

For X-ray image see the spectra in section 6.

\section{1}

$R_{f} 0.4$ (AcOEt/PET 3:7 Anisaldehyde).

${ }^{1} \mathrm{H}$ NMR $\left(\mathrm{CD}_{3} \mathrm{OD}, 400 \mathrm{MHz}\right) \delta 7.71\left(\mathrm{~d}, J=8.1 \mathrm{~Hz}, 1 \mathrm{H} ; \mathrm{H}_{\mathrm{h}}\right), 7.63\left(\mathrm{~d}, J=8.4 \mathrm{~Hz}, 1 \mathrm{H} ; \mathrm{H}_{\mathrm{m}}\right), 7.43$ $\left(\mathrm{t}, J=7.9 \mathrm{~Hz}, 1 \mathrm{H} ; \mathrm{H}_{\mathrm{i}}\right.$ or $\left.\mathrm{H}_{\mathrm{l}}\right), 7.28\left(\mathrm{~s}, 1 \mathrm{H} ; \mathrm{H}_{\mathrm{g}}\right), 7.18\left(\mathrm{t}, J=7.7 \mathrm{~Hz}, 1 \mathrm{H} ; \mathrm{H}_{\mathrm{i}}\right.$ or $\left.\mathrm{H}_{\mathrm{l}}\right), 5.22\left(\mathrm{~s}, 1 \mathrm{H} ; \mathrm{H}_{\mathrm{f}}\right)$, $3.36\left(\mathrm{~d}, J=17.6 \mathrm{~Hz}, 1 \mathrm{H} ; \mathrm{H}_{\mathrm{e}}{ }^{2}\right), 3.12\left(\mathrm{~m}, 1 \mathrm{H} ; \mathrm{H}_{\mathrm{a}}{ }^{2}\right), 2.97\left(\mathrm{~m}, 1 \mathrm{H} ; \mathrm{H}_{\mathrm{a}}{ }^{1}\right), 2.32(\mathrm{~d}, J=17.6 \mathrm{~Hz}, 1 \mathrm{H}$; $\left.\mathrm{H}_{\mathrm{e}}{ }^{1}\right), 1.87-1.60\left(\mathrm{~m}, 5 \mathrm{H} ; \mathrm{H}_{\mathrm{b}}{ }^{1} ; \mathrm{H}_{\mathrm{b}}{ }^{2} ; \mathrm{H}_{\mathrm{c}}{ }^{1} ; \mathrm{H}_{\mathrm{c}}{ }^{2}\right.$ and $\left.\mathrm{NH}\right), 1.31\left(\mathrm{q}, J=7.6 \mathrm{~Hz}, 2 \mathrm{H} ; 2 \mathrm{H}_{\mathrm{n}}\right), 0.80(\mathrm{t}, J=$ $7.5 \mathrm{~Hz}, 3 \mathrm{H} ; 3 \mathrm{H}_{\mathrm{o}}$ ).

${ }^{13} \mathrm{C}$ NMR $\left(\mathrm{CD}_{3} \mathrm{OD}, 100 \mathrm{MHz}\right) \delta 192.9,138.5,133.1,128.6,127.5,124.3,122.8,111.7,107.4$, 70.7, 45.9, 41.4, 40.2, 33.6, 31.6, 22.1, 7.4.

IR v (cm-1): 2934 (w), 2861 (w), 1676 (s), 1532 (s), 1446 (w), 1407 (w), 1360 (w), 1322 (m), 1247 (w), 1196 (w), 1173 (w), 1146 (w), 1119 (w), 1044 (w), 929 (w), 894 (w), 872 (w), 846 (w), $811(\mathrm{w}), 740(\mathrm{~m}), 634(\mathrm{w})$.

HRMS(ESI) calcd for $\mathrm{C}_{17} \mathrm{H}_{21} \mathrm{~N}_{2} \mathrm{O}^{+}(\mathrm{M}+\mathrm{H})$ 269.1654, found 269.1652.

Important correlations NOESY: $\mathrm{H}_{\mathrm{f}}-\mathrm{H}_{\mathrm{a}}{ }^{1} ; \mathrm{H}_{\mathrm{f}}-2 \mathrm{H}_{\mathrm{n}} ; \mathrm{H}_{\mathrm{f}}-3 \mathrm{H}_{\mathrm{o}} ; \mathrm{H}_{\mathrm{f}}-\mathrm{H}_{\mathrm{e}}{ }^{1} ; \mathrm{H}_{\mathrm{f}}-\mathrm{H}_{\mathrm{m}}$ (see the 2D spectra in section 6) 


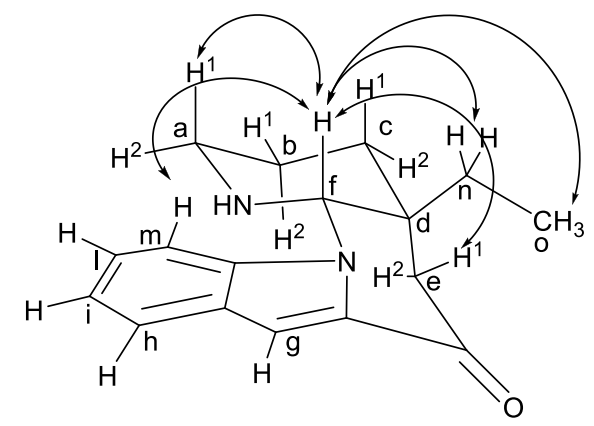

\section{Benzyl 4a-ethyl-6-oxo-2,3,4,4a,5,6,8,9,10,10b-decahydro-1H-pyrano[2,3-h]quinoline-1-} carboxylate (61)

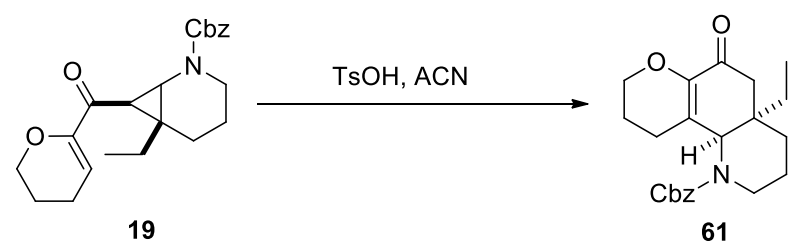

The reaction was performed following GP4, starting from cyclopropane 19 (22 mg, $60 \mu \mathrm{mol}, 1.0$ equiv) and tosic acid ( $2.3 \mathrm{mg}, 12 \mu \mathrm{mol}, 0.2$ equiv). The reaction was quenched after $10 \mathrm{~min}$ and purified on flash chromatography (AcOEt/PET 3:7) to yield 61 (17 $\mathrm{mg}, 46 \mu \mathrm{mol} 78 \%$ ) as colorless oil.

$R_{f} 0.3$ (PET/AcOEt 7:3, Anisaldehyde).

${ }^{1} \mathrm{H}$ NMR $\left(\mathrm{CDCl}_{3}, 400 \mathrm{MHz}\right) \delta$ 7.42-7.30 (m, 5H; $\left.\mathrm{H}_{\mathrm{m}}\right), 5.17\left(\mathrm{~m}, 2 \mathrm{H} ; \mathrm{H}_{\mathrm{l}}\right), 5.04\left(\mathrm{~s}, 0.5 \mathrm{H} ; \mathrm{H}_{\mathrm{f}}\right.$ rotamer A), $4.86\left(\mathrm{~s}, 0.5 \mathrm{H} ; \mathrm{H}_{\mathrm{f}}\right.$ rotamer $\left.\mathrm{B}\right), 4.30-4.17\left(\mathrm{~m}, 1.5 \mathrm{H} ; \mathrm{H}_{\mathrm{i}}\right.$ and $\mathrm{H}_{\mathrm{a}}$ rotamer $\left.\mathrm{A}\right), 4.11(\mathrm{~m}$, $0.5 \mathrm{H} ; \mathrm{H}_{\mathrm{a}}$ rotamer $\left.\mathrm{B}\right), 3.82(\mathrm{~m}, 1 \mathrm{H} ; \mathrm{Hi}), 2.56\left(\mathrm{~m}, 1 \mathrm{H} ; \mathrm{H}_{\mathrm{a}}\right), 2.41\left(\mathrm{~m}, 1 \mathrm{H} ; \mathrm{H}_{\mathrm{e}}^{2}\right), 2.32\left(\mathrm{~m}, 1 \mathrm{H} ; \mathrm{H}_{\mathrm{e}}{ }^{1}\right)$, 2.12-1.75 (m, 4H; $\mathrm{H}_{\mathrm{h}}$ and $\left.\mathrm{H}_{\mathrm{g}}\right), 1.64\left(\mathrm{~m}, 2 \mathrm{H} ; \mathrm{H}_{\mathrm{b}}\right), 1.53(\mathrm{~m}, 2 \mathrm{H} ; \mathrm{Hn}), 1.41\left(\mathrm{~m}, 2 \mathrm{H} ; \mathrm{H}_{\mathrm{c}}\right), 0.85(\mathrm{t}, J=$ $7.5 \mathrm{~Hz}, 1.5 \mathrm{H} ; \mathrm{H}_{\mathrm{o}}$ rotamer A), 0.78 (t, $J=7.6 \mathrm{~Hz}, 1.5 \mathrm{H} ; \mathrm{H}_{\mathrm{o}}$ rotamer $\left.\mathrm{B}\right)$.

${ }^{13} \mathrm{C} \mathrm{NMR}\left(\mathrm{CDCl}_{3}, 100 \mathrm{MHz}\right)$ (rotamers!) $\delta 192.3,192.1,156.3,156.0,146.5,136.6,136.3$, 128.5, 128.5, 128.3, 128.1, 127.7, 126.8, 126.4, 67.6, 67.4, 65.8, 65.7, 58.4, 58.1, 47.1, 47.0, $40.2,39.9,39.0,39.0,28.5,27.4,27.2,22.1,22.0,21.4,20.7,20.2,7.6,7.5$.

IR v (cm-1): 2953 (w), 2938 (w), 2937 (w), 2868 (w), 1688 (s), 1634 (w), 1519 (w), 1462 (w), 1444 (w), 1426 (m), 1386 (w), 1349 (w), 1314 (w), 1298 (w), 1271 (m), 1240 (w), 1209 (w), 


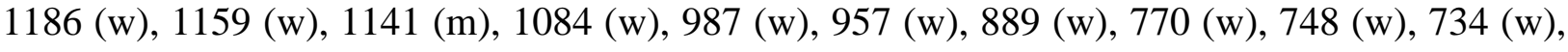
$728(w), 700(w), 677(w), 656(w), 634(m), 607(w)$.

HRMS(ESI) calcd for $\mathrm{C}_{22} \mathrm{H}_{28} \mathrm{NO}_{4}{ }^{+}(\mathrm{M}+\mathrm{H}) 370.2018$, found 370.2012.

Important correlations NOESY: $\mathrm{H}_{\mathrm{f}}-2 \mathrm{H}_{\mathrm{n}} ; \mathrm{H}_{\mathrm{f}}-\mathrm{H}_{\mathrm{e}}{ }^{1}$ (see the 2D spectra in section 6)

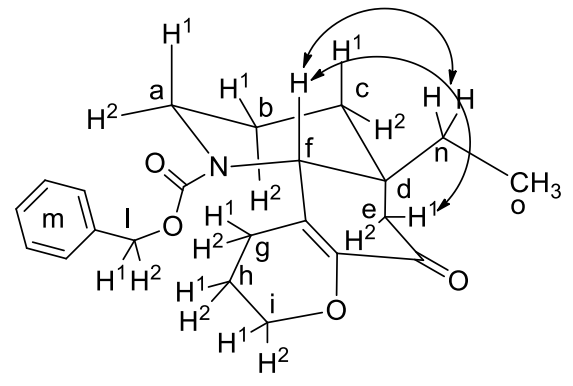

Table 2. Cyclization of acyclic carbamate 21 .

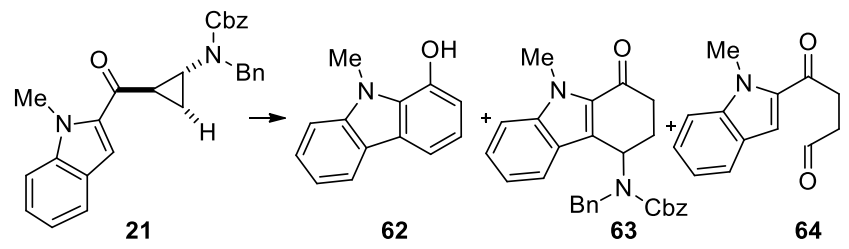

Cyclopropane 21 (10-50 mg, 20-120 $\mu \mathrm{mol})$ was dissolved on the indicated solvent (0.02 M). The indicated catalyst was added and the solution was stirred at $\mathrm{rt}$ during the indicated time. The reaction was quenched with a saturated solution of $\mathrm{NaHCO}_{3}$, extracted with $\mathrm{Et}_{2} \mathrm{O}(3 \mathrm{x})$, dried over $\mathrm{Na}_{2} \mathrm{SO}_{4}$ and concentrated on reduced pressure.

Scale of reactions with cyclopropane $\mathbf{2 1}$ is the following:

Entry 1, 2, 3, 5, 7, 8: 21 (10 mg, $22 \mu \mathrm{mol})$.

Entry 4, 6: 21 (40 mg, $91 \mu \mathrm{mol})$.

9-Methyl-9H-carbazol-1-ol (62)<smiles>Cn1c(C(=O)[C@@H]2C[C@H]2N(Cc2ccccc2)C(=O)OCc2ccccc2)cc2ccccc21</smiles>

21
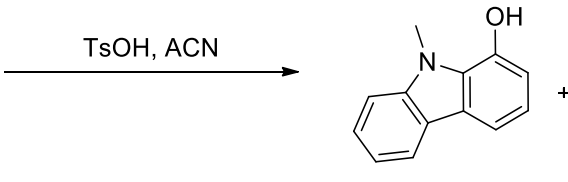

62

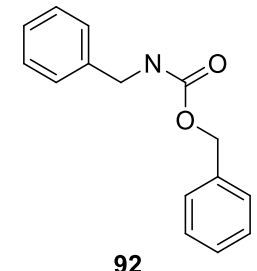

92 
The reaction was performed following GP4, starting from cyclopropane 21 (40 mg, $91 \mu \mathrm{mol}, 1.0$ equiv) and tosic acid ( $3.5 \mathrm{mg}, 18 \mu \mathrm{mol}, 0.2$ equiv). The reaction was quenched after $30 \mathrm{~min}$ and purified on flash chromatography (PET/AcOEt 8:2) affording 62 and $N$-benzyl-benzylcarbamate 92 as inseparable mixture (26 mg, 62/92 1:2.5 molar ratio, 1:3 mass ratio, 36\% yield of 62 by NMR estimation).

$R_{f}$ 0.5 (AcOEt/PET 3:7 Anisaldehyde).

${ }^{1} \mathrm{H}$ NMR (acetone-d $\left.6,400 \mathrm{MHz}\right) \delta 8.73(\mathrm{~s}, 1 \mathrm{H} ; \mathrm{OH}), 8.09$ (m, 1H; C(5)-H), 7.66 (dd, J = 7.6, 0.9 Hz, 1H; C(4)-H), 7.53-7.44 (m, 2H; C(7)-H, C(8)-H), 7.20 (m, 1H; C(6)-H), 7.01 (t, J = 7.7 Hz, $1 \mathrm{H} ; \mathrm{C}(3)-\mathrm{H}), 6.94$ (dd, $J=7.8,0.9 \mathrm{~Hz}, 1 \mathrm{H} ; \mathrm{C}(2)-\mathrm{H}), 4.23$ (s, 3H; $\mathrm{NCH}_{3}$ ).

The obtained values for ${ }^{1} \mathrm{H}$ NMR fitted perfectly with the reported ones. ${ }^{[34]}$

\section{4-(Benzylamino)-9-methyl-2,3,4,9-tetrahydro-1H-carbazol-1-one (93)}

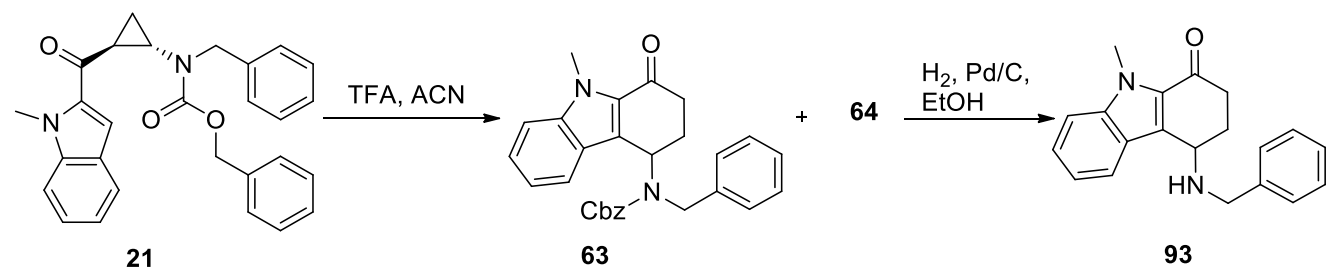

TFA (1.4 $\mu \mathrm{L}, 18 \mu \mathrm{mol}, 0.2$ equiv) was added to a solution of indole 21 (40 mg, $91 \mu \mathrm{mol}, 1.0$ equiv) in distilled acetonitrile $(4 \mathrm{~mL})$. The reaction was stirred at $\mathrm{rt}$ during $1.5 \mathrm{~h}$ then quenched with a saturated aqueous solution of $\mathrm{NaHCO}_{3}(4 \mathrm{~mL})$, extracted with $\mathrm{Et}_{2} \mathrm{O}(3 \mathrm{x} 4 \mathrm{~mL})$ dried over $\mathrm{MgSO}_{4}$ and concentrated under reduced pressure. The crude oil was obtained as a mix of product $63\left(\mathrm{R}_{\mathrm{f}}=0.6 \mathrm{AcOEt} /\right.$ pentane 8:2) and aldehyde 64 (38 mg, 63/64 7:1 molar ratio, 14:1 mass ratio, $89 \%$ by NMR estimation). Indole $\mathbf{6 3}$ was deprotected following the general procedure GP5 to

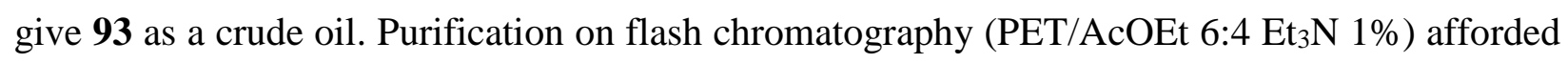
$\mathbf{9 3}$ as a yellow oil (10 mg, $33 \mu \mathrm{mol}, 36 \%)$.

$R_{f} 0.5$ (AcOEt/PET 4:6 Et ${ }_{3} \mathrm{~N} 1 \%$ Anisaldehyde).

${ }^{1} \mathrm{H}$ NMR $\left(\mathrm{CDCl}_{3}, 400 \mathrm{MHz}\right) \delta 7.76(\mathrm{~d}, J=8.1 \mathrm{~Hz}, 1 \mathrm{H} ; \mathrm{ArH}), 7.45-7.32(\mathrm{~m}, 6 \mathrm{H} ; \mathrm{ArH}), 7.29(\mathrm{~m}$, 1H; $\operatorname{ArH}), 7.14(\mathrm{~m}, 1 \mathrm{H} ; \mathrm{ArH}), 4.40(\mathrm{t}, J=4.4 \mathrm{~Hz}, 1 \mathrm{H} ; \mathrm{CHNH}), 4.08\left(\mathrm{~s}, 3 \mathrm{H} ; \mathrm{NCH}_{3}\right), 4.04(\mathrm{~d}, J=$

[34] Oliveira, M. M.; Carvalho, L. M.; Moustrou, C.; Samat, A.; Guglielmetti, R.; Oliveira-Campos, A. M. F., Helv. Chim. Acta 2001, 84, 1163-1171. 
13.2 Hz, 1H; $\mathrm{CH}_{2} \mathrm{Ar}$ ), 3.93 (d, $J=13.2 \mathrm{~Hz}, 1 \mathrm{H} ; \mathrm{CH}_{2} \mathrm{Ar}$ ), 3.05 (ddd, $J=16.9,10.4,4.6 \mathrm{~Hz}, 1 \mathrm{H}$; $\left.\mathrm{CH}_{2}\right), 2.53\left(\mathrm{~m}, 1 \mathrm{H} ; \mathrm{CH}_{2}\right), 2.36\left(\mathrm{~m}, 1 \mathrm{H} ; \mathrm{CH}_{2}\right), 2.27\left(\mathrm{~m}, 1 \mathrm{H} ; \mathrm{CH}_{2}\right)$.

${ }^{13} \mathrm{C}$ NMR $\left(\mathrm{CDCl}_{3}, 100 \mathrm{MHz}\right) \delta 192.4,140.5,129.6,128.5,128.1,127.1,126.5,124.3,121.8$, $120.4,110.3,51.8,49.9,36.1,31.5,30.2 .^{[35]}$

IR v (cm-1): 3466 (w), 3385 (w), $3384(w), 3383(w), 3371(w), 3361(w), 2950(w), 2949(w)$, 2937 (w), 1657 (s), 1615 (w), 1530 (w), 1520 (w), 1494 (w), 1474 (w), 1473 (w), 1456 (w), 1430 (w), 1413 (w), 1412 (w), 1355 (w), 1317 (w), 1247 (w), 1165 (w), 1158 (w), 1132 (w), 1125 (w), $1124(\mathrm{w}), 1074(\mathrm{w}), 1009(\mathrm{w}), 911(\mathrm{~m}), 744(\mathrm{~s}), 702(\mathrm{w}), 692(\mathrm{w}), 652(\mathrm{w}), 642(\mathrm{~m}), 631(\mathrm{~s}), 615$ (m).

HRMS(ESI) calcd for $\mathrm{C}_{20} \mathrm{H}_{21} \mathrm{~N}_{2} \mathrm{O}^{+}(\mathrm{M}+\mathrm{H}) 305.1654$, found 305.1667.

\section{4-(1-Methyl-1H-indol-2-yl)-4-oxobutanal (64)}

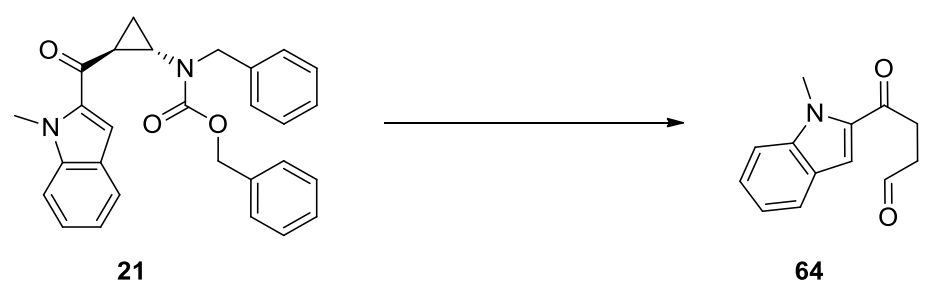

The aldehyde 64 was obtained as major compound in the cyclization reactions carried out in presence of trace of water.

$R_{f} 0.6$ (AcOEt/PET 3:7 Anisaldehyde).

${ }^{1} \mathrm{H}$ NMR $\left(\mathrm{CDCl}_{3}, 400 \mathrm{MHz}\right) \delta 9.92(\mathrm{~s}, 1 \mathrm{H} ; \mathrm{CHO}), 7.70(\mathrm{dt}, J=8.0,0.9 \mathrm{~Hz}, 1 \mathrm{H} ; \mathrm{ArH}), 7.39(\mathrm{~m}$, $3 \mathrm{H} ; \mathrm{ArH}), 7.16(\mathrm{~m}, 1 \mathrm{H} ; \mathrm{ArH}), 4.06\left(\mathrm{~s}, 3 \mathrm{H} ; \mathrm{NCH}_{3}\right), 3.38$ (t, $\left.J=6.3 \mathrm{~Hz}, 2 \mathrm{H} ; \mathrm{CH}_{2} \mathrm{CO}\right), 2.92(\mathrm{t}, J=$ $\left.6.5 \mathrm{~Hz}, 2 \mathrm{H} ; \mathrm{CH}_{2} \mathrm{CHO}\right)$.

${ }^{13} \mathrm{C}$ NMR $\left(\mathrm{CDCl}_{3}, 100 \mathrm{MHz}\right) \delta 200.7,191.6,128.1,127.5,126.0,125.8,122.9,120.8,111.5$, $110.4,37.8,32.2,32.0$.

IR v (cm-1): 2922 (w), 2907 (w), 2819 (w), 2717 (w), 1729 (m), 1661 (s), 1611 (w), 1514 (m), 1466 (m), 1410 (m), 1392 (m), 1355 (w), 1320 (w), 1284 (m), 1168 (m), 1127 (w), 995 (m), 912 (w), $807(\mathrm{~m}), 754(\mathrm{~s}), 745(\mathrm{~m}), 680(\mathrm{w}), 634(\mathrm{w})$.

[35] Not all the aromatic signals were resolved 
HRMS(ESI) calcd for $\mathrm{C}_{13} \mathrm{H}_{14} \mathrm{NO}_{2}^{+}(\mathrm{M}+\mathrm{H}) 216.1024$, found 216.1025.

Table 3. Selective cyclization of acyclic carbamate 22.

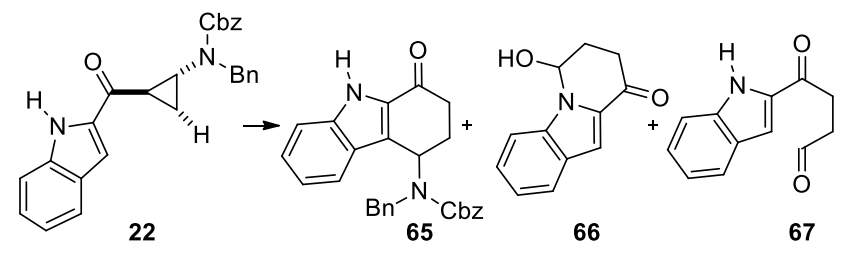

Cyclopropane 21 (5-30 mg, 12-70 $\mu \mathrm{mol})$ was dissolved on the indicated solvent $(0.02 \mathrm{M})$. The indicated catalyst was added and the solution was stirred at $\mathrm{rt}$ during the indicated time. The reaction was quenched with a saturated solution of $\mathrm{NaHCO}_{3}$, extracted with $\mathrm{Et}_{2} \mathrm{O}(3 \mathrm{x})$, dried over $\mathrm{Na}_{2} \mathrm{SO}_{4}$ and concentrated on reduced pressure.

Scale of reactions with cyclopropane $\mathbf{2 2}$ is the following:

Entry 1: 22 (5 mg, $12 \mu \mathrm{mol})$.

Entry 2: 22 (30 mg, $71 \mu \mathrm{mol})$.

Entry 3: 22 (30 mg, $71 \mu \mathrm{mol})$.

Benzyl benzyl(1-oxo-2,3,4,9-tetrahydro-1H-carbazol-4-yl)carbamate (65)

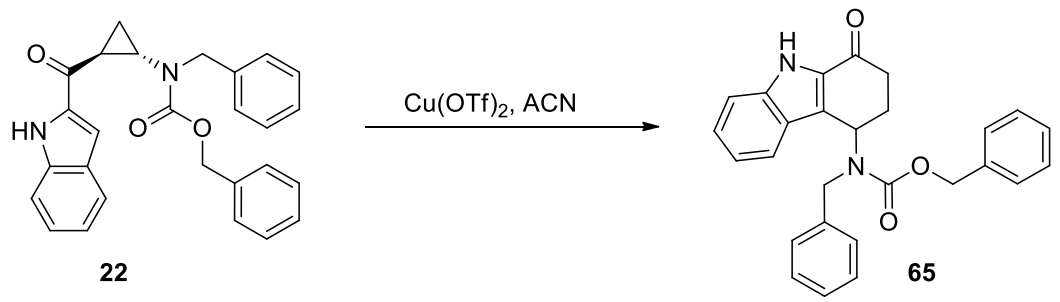

$\mathrm{Cu}(\mathrm{OTf})_{2}(4.7 \mathrm{mg}, 13 \mu \mathrm{mol}, 0.2$ equiv) was added to a solution of indole 22 (28 $\mathrm{mg}, 66 \mu \mathrm{mol}$, 1.0 equiv) in distilled acetonitrile $(3 \mathrm{~mL})$. The reaction was stirred at $\mathrm{rt}$ during $10 \mathrm{~min}$ then quenched with a saturated aqueous solution of $\mathrm{NaHCO}_{3}(3 \mathrm{~mL})$, extracted with $\mathrm{Et}_{2} \mathrm{O}(3 \mathrm{x} 4 \mathrm{~mL})$ dried over $\mathrm{MgSO}_{4}$ and concentrated under reduced pressure. The crude product $(70 \%$ by NMR estimation) was purified on flash chromatography (PET/AcOEt 8:2 on deactivated silica) affording $\mathbf{6 5}$ as a yellow oil with tendency to aromatize (12 mg, $28 \mu \mathrm{mol}, 43 \%)$.

$R_{f} 0.5(\mathrm{AcOEt} / \mathrm{PET} 3: 7)$

${ }^{1} \mathrm{H} \mathrm{NMR}\left(\mathrm{CDCl}_{3}, 400 \mathrm{MHz}\right) \delta 8.78(\mathrm{~m}, 1 \mathrm{H} ; \mathrm{NH}), 7.47-7.15(\mathrm{~m}, 12 \mathrm{H} ; \mathrm{ArH}), 7.08(\mathrm{~m}, 2 \mathrm{H} ; \mathrm{ArH})$, 6.15 (m, 0.6H; CHN rotamer A), $5.83\left(\mathrm{~m}, 0.4 \mathrm{H}\right.$; CHN rotamer B), 5.42-5.16 (m, 2H; $\left.\mathrm{OCH}_{2} \mathrm{Ar}\right)$, 
$4.86\left(\mathrm{~m}, 0.4 \mathrm{H} ; \mathrm{CH}_{2} \mathrm{Ar}\right.$ rotamer B), 4.77 (m, 0.6H; $\mathrm{CH}_{2} \mathrm{Ar}$ rotamer A), $4.11\left(\mathrm{~m}, 0.4 \mathrm{H} ; \mathrm{CH}_{2} \mathrm{Ar}\right.$ rotamer B), 4.00 (m, 0.6H; $\mathrm{CH}_{2} \mathrm{Ar}$ rotamer A), $2.59\left(\mathrm{~m}, 2 \mathrm{H} ; \mathrm{CNCH}_{2}\right), 2.25\left(\mathrm{~m}, 2 \mathrm{H} ; \mathrm{COCH}_{2}\right)$.

${ }^{13} \mathrm{C}$ NMR $\left(\mathrm{CDCl}_{3}, 100 \mathrm{MHz}\right) \delta 189.9,156.8,139.0,137.6,136.4,132.0,128.5,128.1,127.2$, $126.9,126.4,126.2,125.0,121.6,121.5,112.6,67.7,52.1,47.4,36.7,30.8 .^{[36]}$

IR v (cm-1): $3324(\mathrm{w}), 3283(\mathrm{w}), 3274(\mathrm{w}), 3262(\mathrm{w}), 3250(\mathrm{w}), 3064(\mathrm{w}), 3033(\mathrm{w}), 2952(\mathrm{w})$, 2937 (w), 1699 (s), 1660 (s), 1537 (m), 1471 (m), 1455 (m), 1454 (m), 1413 (m), 1364 (m), 1346 (m), 1332 (m), 1289 (m), 1252 (m), 1211 (m), 1114 (m), 1079 (w), 1048 (w), 1030 (w), 960 (w), $921(\mathrm{w}), 737(\mathrm{~s}), 699(\mathrm{~s}), 628(\mathrm{~m})$.

HRMS(ESI) calcd for $\mathrm{C}_{27} \mathrm{H}_{25} \mathrm{~N}_{2} \mathrm{O}_{3}{ }^{+}(\mathrm{M}+\mathrm{H})$ 425.1865, found 425.1877.

\section{6-Hydroxy-7,8-dihydropyrido[1,2-a]indol-9(6H)-one (66)}

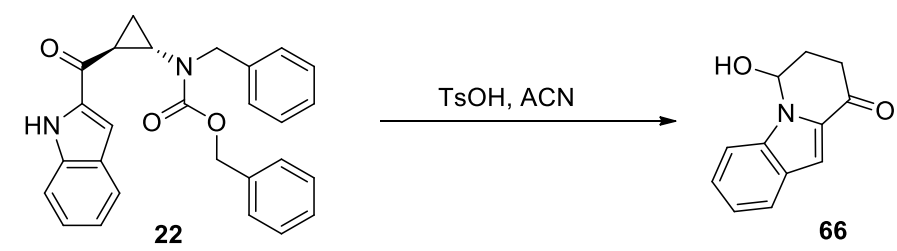

The reaction was performed following GP4, starting from cyclopropane 22 ( $28 \mathrm{mg}, 66 \mu \mathrm{mol}, 1.0$ equiv) and tosic acid ( $2.5 \mathrm{mg}, 13 \mu \mathrm{mol}, 0.2$ equiv). The reaction was quenched after $30 \mathrm{~min}$. The crude product was purified on flash chromatography (PET/AcOEt 8:2 on deactivated silica) affording 66 as a yellow oil (10 mg, $50 \mu \mathrm{mol}, 75 \%)$.

$R_{f} 0.5(\mathrm{AcOEt} / \mathrm{PET} 3: 7)$

${ }^{1} \mathrm{H} \mathrm{NMR}\left(\mathrm{CDCl}_{3}, 400 \mathrm{MHz}\right) \delta 7.74(\mathrm{~d}, J=8.1 \mathrm{~Hz}, 1 \mathrm{H} ; \mathrm{ArH}), 7.51(\mathrm{~m}, 1 \mathrm{H} ; \mathrm{ArH}), 7.42(\mathrm{~m}, 1 \mathrm{H}$; ArH), 7.31 (s, 1H; ArH), $7.21(\mathrm{~m}, 1 \mathrm{H} ; \mathrm{ArH}), 6.23$ (t, $J=2.8 \mathrm{~Hz}, 1 \mathrm{H} ; \mathrm{CH}-\mathrm{OH}), 3.10$ (m, 1H; $\left.\mathrm{CH}_{2}\right), 2.68\left(\mathrm{~m}, 1 \mathrm{H} ; \mathrm{CH}_{2}\right), 2.41-2.59\left(\mathrm{~m}, 2 \mathrm{H} ; \mathrm{CH}_{2}\right)$.

${ }^{13} \mathrm{C}$ NMR $\left(\mathrm{CDCl}_{3}, 100 \mathrm{MHz}\right) \delta 190.1,136.5,132.4,127.2,126.4,123.7,121.8,110.2,107.3$, $72.9,31.6,30.2$.

IR v (cm-1): 3348 (w), 3068 (w), 2958 (w), 1673 (s), 1530 (s), 1446 (m), 1404 (m), 1354 (m), 1328 (m), 1249 (m), 1207 (m), 1168 (m), 1141 (m), 1066 (m), 1026 (m), 955 (m), 910 (m), 814 (m), $742(\mathrm{~s}), 701(\mathrm{~m}), 673(\mathrm{~m})$

[36] Not all the aromatic signals were resolved 
HRMS(ESI) calcd for $\mathrm{C}_{12} \mathrm{H}_{12} \mathrm{NO}_{2}{ }^{+}(\mathrm{M}+\mathrm{H})$ 202.0868, found 202.0862.

\section{3,4,4a,5-Tetrahydro-2H-pyrano[3',2':5,6]pyrido[1,2-a]indol-6(12aH)-one (68)}

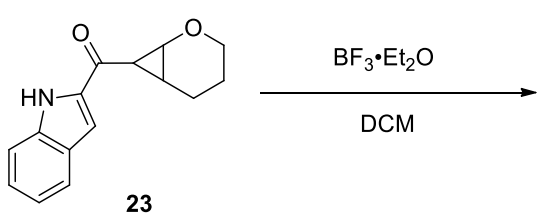

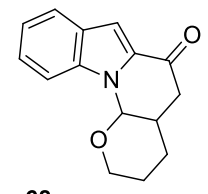

68

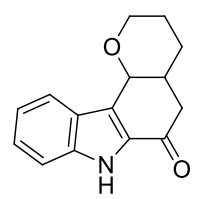

94

$\mathrm{BF}_{3} \cdot \mathrm{Et}_{2} \mathrm{O}(2.0 \mu \mathrm{L}, 16 \mu \mathrm{mol}, 0.20$ equiv) was added to a solution of indole 23 (18 $\mathrm{mg}, 75 \mu \mathrm{mol}$, 1.0 equiv) in distilled DCM $(5 \mathrm{~mL})$. The reaction was stirred at $\mathrm{rt}$ during 15 min then quenched with a saturated aqueous solution of $\mathrm{NaHCO}_{3}(3 \mathrm{~mL})$, extracted with $\mathrm{Et}_{2} \mathrm{O}(3 \mathrm{x} 4 \mathrm{~mL})$ dried over $\mathrm{MgSO}_{4}$ and concentrated under reduced pressure. The crude product was purified on flash chromatography (PET/AcOEt 7:3) to afford 68 and its regioisomer 95 (as pink oil (14 mg, 58 $\mu \mathrm{mol}, 65 \% 68$ and $14 \%$ 95). The pure 68 was obtained resubmitting the mixture of regioisomers to stoichiometric amount of $\mathrm{BF}_{3} \cdot \mathrm{Et}_{2} \mathrm{O}$ during 24 hours.

68

$R_{f} 0.3($ AcOEt/PET 3:7).

${ }^{1} \mathrm{H}$ NMR $\left(\mathrm{CDCl}_{3}, 400 \mathrm{MHz}\right) \delta 7.72(\mathrm{dt}, J=8.1,0.9 \mathrm{~Hz}, 1 \mathrm{H}$; $\mathrm{ArH}), 7.51(\mathrm{dd}, J=8.4,0.8 \mathrm{~Hz}, 1 \mathrm{H}$; $\operatorname{ArH}), 7.41(\mathrm{~m}, 1 \mathrm{H} ; \operatorname{ArH}), 7.32(\mathrm{~m}, 1 \mathrm{H} ; \operatorname{ArH}), 7.20(\mathrm{~m}, 1 \mathrm{H} ; \operatorname{ArH}), 5.72\left(\mathrm{~d}, J=2.7 \mathrm{~Hz}, 1 \mathrm{H} ; \mathrm{H}_{\mathrm{f}}\right)$, $4.04\left(\mathrm{~m}, 1 \mathrm{H} ; \mathrm{H}_{\mathrm{a}}{ }^{2}\right), 3.82\left(\mathrm{~m}, 1 \mathrm{H} ; \mathrm{H}_{\mathrm{a}}{ }^{1}\right), 3.16\left(\mathrm{dd}, J=16.5,12.4 \mathrm{~Hz}, 1 \mathrm{H} ; \mathrm{H}_{\mathrm{e}}{ }^{1}\right), 2.62\left(\mathrm{~m}, 1 \mathrm{H}, \mathrm{H}_{\mathrm{d}}\right)$, $2.53\left(\mathrm{~m}, 1 \mathrm{H}\right.$ dd, $\left.J=16.5,4.1 \mathrm{~Hz}, 1 \mathrm{H} ; \mathrm{H}_{\mathrm{e}}{ }^{2}\right), 2.08\left(\mathrm{~m}, 1 \mathrm{H} ; \mathrm{H}_{\mathrm{b}}{ }^{1}\right), 1.89\left(\mathrm{~m}, 2 \mathrm{H} ; \mathrm{H}_{\mathrm{c}}{ }^{1-2}\right), 1.58(\mathrm{~m}, 1 \mathrm{H}$; $\mathrm{H}_{\mathrm{b}}^{2}$.

${ }^{13} \mathrm{C} \mathrm{NMR}\left(\mathrm{CDCl}_{3}, 100 \mathrm{MHz}\right) \delta 190.1,137.1,132.4,127.3,126.3,123.5,121.8,110.9,106.9$, $80.3,66.8,37.7,33.9,27.3,20.4$.

IR v (cm-1): 3297 (w), $2852(\mathrm{w}), 1678$ (s), 1619 (w), 1593 (w), 1532 (s), 1485 (m), 1449 (m), 1383 (m), 1367 (m), 1342 (m), 1325 (s), 1248 (m), 1223 (m), 1211 (m), 1184 (m), 1170 (m), 1146 (m), 1090 (m), 1068 (m), 1047 (s), 994 (w), 938 (m), 919 (m), 905 (m), 904 (m), 821 (m), $808(\mathrm{~m}), 754(\mathrm{~s}), 636(\mathrm{~s}), 627(\mathrm{~m})$.

HRMS(ESI) calcd for $\mathrm{C}_{15} \mathrm{H}_{16} \mathrm{NO}_{2}^{+}(\mathrm{M}+\mathrm{H})$ 242.1176, found 242.1182.

Important correlations ROESY: $\mathrm{H}_{\mathrm{f}}-\mathrm{H}_{\mathrm{d}}, \mathrm{H}_{\mathrm{f}}-\mathrm{H}_{\mathrm{c}}{ }^{1}, \mathrm{H}_{\mathrm{f}}-\mathrm{H}_{\mathrm{b}}{ }^{1}, \mathrm{H}_{\mathrm{f}}-\mathrm{H}_{\mathrm{e}}{ }^{1}$ (see $2 \mathrm{D}$ spectra in section 6) 


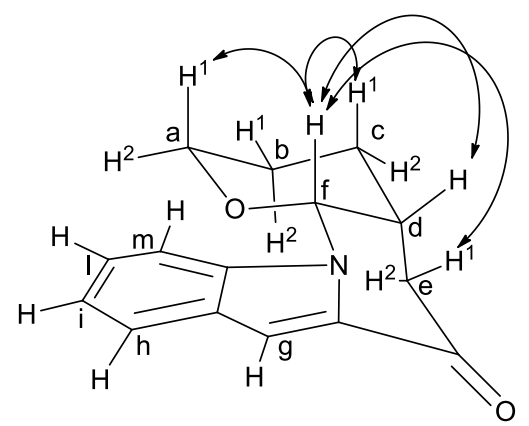

\section{Computational data}

\section{Cartesian coordinates for $57-60$}

\section{7}

56

scf done: -1303.604044
C $\quad 2.243115 \quad 0.145332$
$-0.159380$
$\begin{array}{llll}\text { C } & 3.541420 & -0.206153 & 0.178326\end{array}$
$\begin{array}{llll}\text { C } & -1.129513 & -0.230333 & -0.924092\end{array}$
$\begin{array}{llll}\text { O } & -0.977974 & -0.278499 & -2.147026\end{array}$
$\begin{array}{llll}\text { O } & -2.316581 & 0.086257 & -0.345740\end{array}$
$\begin{array}{llll}\text { C } & -3.412862 & 0.375868 & -1.260717\end{array}$

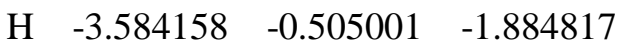
$\begin{array}{llll}\mathrm{H} & -3.117316 & 1.209685 & -1.902626\end{array}$
$\begin{array}{llll}\text { C } & -4.623308 & 0.713396 & -0.431623\end{array}$
$\begin{array}{llll}\text { C } & -4.885519 & 2.040429 & -0.060762\end{array}$
$\begin{array}{llll}\text { C } & -5.502317 & -0.296468 & -0.013310\end{array}$
$\begin{array}{llll}\text { C } & -6.002353 & 2.353001 & 0.719913\end{array}$
$\begin{array}{llll}\mathrm{H} & -4.214700 & 2.831977 & -0.387776\end{array}$
$\begin{array}{llll}\text { C } & -6.619710 & 0.012181 & 0.767795\end{array}$
$\begin{array}{llll}\mathrm{H} & -5.312348 & -1.327501 & -0.303740\end{array}$
$\begin{array}{llll}\text { C } & -6.871020 & 1.338363 & 1.136397\end{array}$
H $\quad-6.196250 \quad 3.385576 \quad 0.998406$
$\begin{array}{llll}\mathrm{H} & -7.294767 & -0.778986 & 1.083566\end{array}$
$\begin{array}{llll}\mathrm{H} & -7.741736 & 1.580385 & 1.740323\end{array}$
$\begin{array}{llll}\text { C } & 2.199471 & 1.579525 & -0.165091\end{array}$
$\begin{array}{llll}\text { C } & 3.510454 & 2.031553 & 0.193597\end{array}$
$\begin{array}{llll}\text { C } & 1.200572 & 2.542408 & -0.437260\end{array}$
$\begin{array}{llll}\text { C } & 3.832092 & 3.395359 & 0.285995\end{array}$
$\begin{array}{llll}\text { C } & 1.520591 & 3.889353 & -0.348219\end{array}$
$\begin{array}{llll}\mathrm{H} & 0.197940 & 2.237050 & -0.717044\end{array}$
$\begin{array}{llll}\text { C } & 2.824711 & 4.311078 & 0.012111\end{array}$
$\begin{array}{llll}\mathrm{H} & 4.832223 & 3.719234 & 0.559852\end{array}$ 


$\begin{array}{crrr}\mathrm{H} & 0.760985 & 4.637305 & -0.558701 \\ \mathrm{H} & 3.040817 & 5.374361 & 0.073556 \\ \mathrm{~N} & 4.298732 & 0.927715 & 0.398514 \\ \mathrm{H} & 5.279833 & 0.923164 & 0.643946 \\ \mathrm{H} & 0.345371 & 0.328357 & 1.860551 \\ \mathrm{C} & -0.343769 & -0.421023 & 1.448322 \\ \mathrm{C} & -0.058201 & -1.791937 & 2.067209 \\ \mathrm{H} & -1.359272 & -0.090755 & 1.655733 \\ \mathrm{C} & 1.183239 & -0.885726 & -0.479068 \\ \mathrm{C} & 1.327079 & -2.301171 & 1.642853 \\ \mathrm{H} & -0.102154 & -1.705506 & 3.159954 \\ \mathrm{H} & -0.844208 & -2.497037 & 1.771498 \\ \mathrm{C} & 1.550142 & -2.298590 & 0.102815 \\ \mathrm{H} & 1.099598 & -0.964743 & -1.566091 \\ \mathrm{H} & 2.085714 & -1.670771 & 2.125014 \\ \mathrm{H} & 1.488484 & -3.319937 & 2.017483 \\ \mathrm{C} & 3.041254 & -2.618965 & -0.181729 \\ \mathrm{H} & 3.316260 & -3.575166 & 0.278478 \\ \mathrm{H} & 3.199427 & -2.732482 & -1.263638 \\ \mathrm{C} & 0.655886 & -3.399514 & -0.535530 \\ \mathrm{H} & 0.835337 & -4.329627 & 0.021294 \\ \mathrm{H} & -0.394416 & -3.142802 & -0.356767 \\ \mathrm{C} & 0.834224 & -3.687484 & -2.034110 \\ \mathrm{H} & 0.096361 & -4.433996 & -2.351147 \\ \mathrm{H} & 0.679197 & -2.797148 & -2.653657 \\ \mathrm{H} & 1.824703 & -4.094030 & -2.266779 \\ \mathrm{C} & 4.035629 & -1.564905 & 0.268908 \\ \mathrm{O} & 5.187062 & -1.838396 & 0.628491 \\ \mathrm{~N} & -0.149052 & -0.467805 & -0.009595\end{array}$

\section{8}

56

scf done: -1303.593543

$\begin{array}{lrrr}\mathrm{C} & -3.553101 & 0.415946 & -0.335030 \\ \mathrm{C} & 1.017901 & -0.346321 & 0.912913 \\ \mathrm{O} & 0.872429 & -0.371901 & 2.134963 \\ \mathrm{O} & 2.227355 & -0.265035 & 0.314078 \\ \mathrm{C} & 3.381429 & -0.195488 & 1.205225 \\ \mathrm{H} & 3.368182 & -1.074094 & 1.854451 \\ \mathrm{H} & 3.284604 & 0.701893 & 1.821623 \\ \mathrm{C} & 4.616711 & -0.153988 & 0.346442 \\ \mathrm{C} & 5.279358 & -1.339316 & -0.003956 \\ \mathrm{C} & 5.116472 & 1.070021 & -0.122329 \\ \mathrm{C} & 6.418060 & -1.304024 & -0.814189 \\ \mathrm{H} & 4.903877 & -2.292799 & 0.361060 \\ \mathrm{C} & 6.253928 & 1.108873 & -0.933570 \\ \mathrm{H} & 4.614526 & 1.995485 & 0.151346 \\ \mathrm{C} & 6.906411 & -0.079111 & -1.281468 \\ \mathrm{H} & 6.924426 & -2.229269 & -1.076515 \\ \mathrm{H} & 6.632874 & 2.063526 & -1.289049 \\ \mathrm{H} & 7.793377 & -0.049802 & -1.909041 \\ \mathrm{C} & -1.958293 & 1.877781 & 0.289996 \\ \mathrm{C} & -3.091444 & 2.618438 & -0.175296 \\ \mathrm{C} & -0.824015 & 2.539344 & 0.798737 \\ \mathrm{C} & -3.067039 & 4.030145 & -0.154314\end{array}$




$\begin{array}{lrrr}\mathrm{C} & -0.834805 & 3.928913 & 0.811193 \\ \mathrm{H} & 0.024826 & 1.999499 & 1.197292 \\ \mathrm{C} & -1.940281 & 4.673729 & 0.332192 \\ \mathrm{H} & -3.925246 & 4.595430 & -0.508656 \\ \mathrm{H} & 0.028303 & 4.457029 & 1.207953 \\ \mathrm{H} & -1.902979 & 5.759342 & 0.359158 \\ \mathrm{H} & -0.428692 & 0.441865 & -1.866894 \\ \mathrm{C} & 0.178895 & -0.372804 & -1.452618 \\ \mathrm{C} & -0.258924 & -1.713867 & -2.041704 \\ \mathrm{H} & 1.222091 & -0.153430 & -1.666999 \\ \mathrm{C} & -1.367082 & -0.611891 & 0.495574 \\ \mathrm{C} & -1.697025 & -2.032426 & -1.612622 \\ \mathrm{H} & -0.201871 & -1.664263 & -3.135749 \\ \mathrm{H} & 0.434453 & -2.499836 & -1.719614 \\ \mathrm{C} & -1.932494 & -1.953947 & -0.075096 \\ \mathrm{H} & -1.299264 & -0.671892 & 1.581968 \\ \mathrm{H} & -2.366229 & -1.330439 & -2.127542 \\ \mathrm{H} & -1.985420 & -3.034476 & -1.953042 \\ \mathrm{C} & -3.451463 & -2.033413 & 0.191364 \\ \mathrm{H} & -3.859543 & -2.975885 & -0.189217 \\ \mathrm{H} & -3.653810 & -2.005432 & 1.271553 \\ \mathrm{C} & -1.212361 & -3.149511 & 0.611779 \\ \mathrm{H} & -1.507616 & -4.054929 & 0.065375 \\ \mathrm{H} & -0.132234 & -3.046757 & 0.457876 \\ \mathrm{C} & -1.475866 & -3.377095 & 2.107987 \\ \mathrm{H} & -0.863522 & -4.215716 & 2.459248 \\ \mathrm{H} & -1.213002 & -2.507432 & 2.720387 \\ \mathrm{H} & -2.521489 & -3.632565 & 2.311609 \\ \mathrm{C} & -4.217082 & -0.881548 & -0.419435 \\ \mathrm{O} & -5.343447 & -1.014044 & -0.905330 \\ \mathrm{~N} & -0.009234 & -0.377637 & 0.008846 \\ \mathrm{~N} & -2.245331 & 0.530687 & 0.155285 \\ \mathrm{C} & -4.080988 & 1.673012 & -0.559820 \\ \mathrm{H} & -5.079739 & 1.874405 & -0.922907\end{array}$

59

56

scf done: -1303.585800

$\begin{array}{lrrr}\mathrm{C} & 0.882426 & 0.692011 & 0.078502 \\ \mathrm{C} & 1.045360 & -0.808517 & 0.085790 \\ \mathrm{C} & 2.428897 & -1.177229 & 0.734788 \\ \mathrm{C} & 2.297930 & -0.693214 & 2.207856 \\ \mathrm{C} & 1.267500 & 1.383705 & 1.220845 \\ \mathrm{H} & 0.305904 & -1.193872 & 0.797748 \\ \mathrm{H} & 1.496730 & -1.258789 & 2.708649 \\ \mathrm{H} & 3.211488 & -0.881751 & 2.780004 \\ \mathrm{C} & 2.530177 & -2.710773 & 0.645485 \\ \mathrm{H} & 1.642453 & -3.139169 & 1.129798 \\ \mathrm{H} & 3.393152 & -3.080053 & 1.210137 \\ \mathrm{C} & 1.864167 & -2.276957 & -1.817655 \\ \mathrm{H} & 1.419379 & -2.854500 & -2.626809 \\ \mathrm{H} & 2.553814 & -1.564596 & -2.279099 \\ \mathrm{C} & 2.602618 & -3.203457 & -0.820931 \\ \mathrm{H} & 2.178850 & -4.212639 & -0.874822 \\ \mathrm{H} & 3.646046 & -3.286698 & -1.147103\end{array}$




\begin{tabular}{|c|c|c|c|}
\hline N & 0.773192 & -1.528672 & \\
\hline$c$ & -0.510434 & 27147 & \\
\hline $\mathrm{O}$ & -0.827951 & 3273 & \\
\hline $\mathrm{O}$ & 2502 & & \\
\hline $\mathrm{C}$ & -2.821506 & -1.488466 & \\
\hline $\mathrm{H}$ & -2.989695 & & \\
\hline $\mathrm{H}$ & -3.017118 & & \\
\hline $\mathrm{C}$ & -3.6 & & \\
\hline $\mathrm{C}$ & -4.0 & & \\
\hline $\mathrm{C}$ & & & \\
\hline $\mathrm{C}$ & -4.7 & & \\
\hline $\mathrm{H}$ & -3.7 & & \\
\hline $\mathrm{C}$ & -4.8 & & \\
\hline $\mathrm{H}$ & -3.8 & & \\
\hline $\mathrm{C}$ & -5.2 & & \\
\hline $\mathrm{H}$ & -5.0 & & \\
\hline $\mathrm{H}$ & -5.1 & & \\
\hline $\mathrm{H}$ & -5.8 & & \\
\hline $\mathrm{C}$ & & & \\
\hline $\mathrm{H}$ & & & \\
\hline $\mathrm{H}$ & & & \\
\hline $\mathrm{C}$ & & & \\
\hline $\mathrm{H}$ & & & \\
\hline $\mathrm{H}$ & & & \\
\hline $\mathrm{H}$ & & & \\
\hline$C$ & & & \\
\hline $\mathrm{O}$ & & & \\
\hline$C$ & & & \\
\hline $\mathrm{C}$ & & & \\
\hline $\mathrm{C}$ & & & \\
\hline $\mathrm{C}$ & & & \\
\hline $\mathrm{C}$ & & & \\
\hline $\mathrm{H}$ & & & \\
\hline $\mathrm{C}$ & & & \\
\hline $\mathrm{H}$ & & & \\
\hline $\mathrm{H}$ & & & \\
\hline $\mathrm{H}$ & & & \\
\hline $\mathrm{N}$ & & & \\
\hline U & & & \\
\hline \\
\hline \multicolumn{4}{|c|}{ scf done: -1303.577653} \\
\hline $\mathrm{C}$ & & & \\
\hline $\mathrm{C}$ & & & \\
\hline $\mathrm{C}$ & & & \\
\hline $\mathrm{C}$ & & & \\
\hline $\mathrm{H}$ & & & \\
\hline $\mathrm{H}$ & & & \\
\hline $\mathrm{H}$ & & & \\
\hline $\mathrm{C}$ & & & \\
\hline $\mathrm{H}$ & & & \\
\hline $\mathrm{H}$ & & & \\
\hline $\mathrm{C}$ & & & \\
\hline $\mathrm{H}$ & 1.489777 & -2.556257 & -2.754 \\
\hline
\end{tabular}




$\begin{array}{lrrr}\mathrm{H} & 2.572045 & -1.253901 & -2.297444 \\ \mathrm{C} & 2.689616 & -3.007209 & -0.978504 \\ \mathrm{H} & 2.339014 & -4.031637 & -1.144866 \\ \mathrm{H} & 3.745828 & -2.985770 & -1.269997 \\ \mathrm{~N} & 0.795288 & -1.384514 & -1.196690 \\ \mathrm{C} & -0.487626 & -1.715295 & -1.571740 \\ \mathrm{O} & -0.772364 & -2.386618 & -2.559248 \\ \mathrm{O} & -1.410932 & -1.186402 & -0.739034 \\ \mathrm{C} & -2.806049 & -1.480429 & -1.054837 \\ \mathrm{H} & -2.925713 & -2.565921 & -1.092187 \\ \mathrm{H} & -3.027311 & -1.065172 & -2.041093 \\ \mathrm{C} & -3.657956 & -0.859344 & 0.018729 \\ \mathrm{C} & -3.934994 & -1.563150 & 1.200228 \\ \mathrm{C} & -4.180798 & 0.431652 & -0.143683 \\ \mathrm{C} & -4.714510 & -0.984576 & 2.205564 \\ \mathrm{H} & -3.541304 & -2.568754 & 1.331433 \\ \mathrm{C} & -4.962087 & 1.012925 & 0.859531 \\ \mathrm{H} & -3.978407 & 0.982490 & -1.059588 \\ \mathrm{C} & -5.228921 & 0.305838 & 2.036681 \\ \mathrm{H} & -4.923995 & -1.540731 & 3.115590 \\ \mathrm{H} & -5.363688 & 2.013327 & 0.720917 \\ \mathrm{H} & -5.838581 & 0.755577 & 2.816059 \\ \mathrm{C} & 3.632406 & -0.357978 & 0.198775 \\ \mathrm{H} & 3.648076 & -0.383277 & -0.893753 \\ \mathrm{H} & 3.523279 & 0.700037 & 0.461452 \\ \mathrm{C} & 4.998676 & -0.842311 & 0.710527 \\ \mathrm{H} & 5.786590 & -0.188358 & 0.319666 \\ \mathrm{H} & 5.069117 & -0.818092 & 1.803751 \\ \mathrm{H} & 5.227168 & -1.861824 & 0.382205 \\ \mathrm{C} & 1.711334 & 0.642655 & 2.473353 \\ \mathrm{O} & 1.815441 & 1.171118 & 3.583751 \\ \mathrm{C} & 0.497649 & 1.655100 & -0.809476 \\ \mathrm{C} & 0.420322 & 2.919549 & -0.134253 \\ \mathrm{C} & 0.184138 & 1.550508 & -2.180206 \\ \mathrm{C} & 0.016494 & 4.072470 & -0.844397 \\ \mathrm{C} & -0.210899 & 2.703852 & -2.844751 \\ \mathrm{H} & 0.264795 & 0.613377 & -2.715898 \\ \mathrm{C} & -0.299487 & 3.957183 & -2.187970 \\ \mathrm{H} & -0.039265 & 5.032163 & -0.336809 \\ \mathrm{H} & -0.452102 & 2.642707 & -3.902850 \\ \mathrm{H} & -0.611425 & 4.832498 & -2.751167 \\ \mathrm{C} & 0.821435 & 2.706866 & 1.212738 \\ \mathrm{H} & 0.911000 & 3.446519 & 1.996920 \\ \mathrm{~N} & 0.935614 & 0.724366 & 0.106489\end{array}$


9 Spectra of new compounds
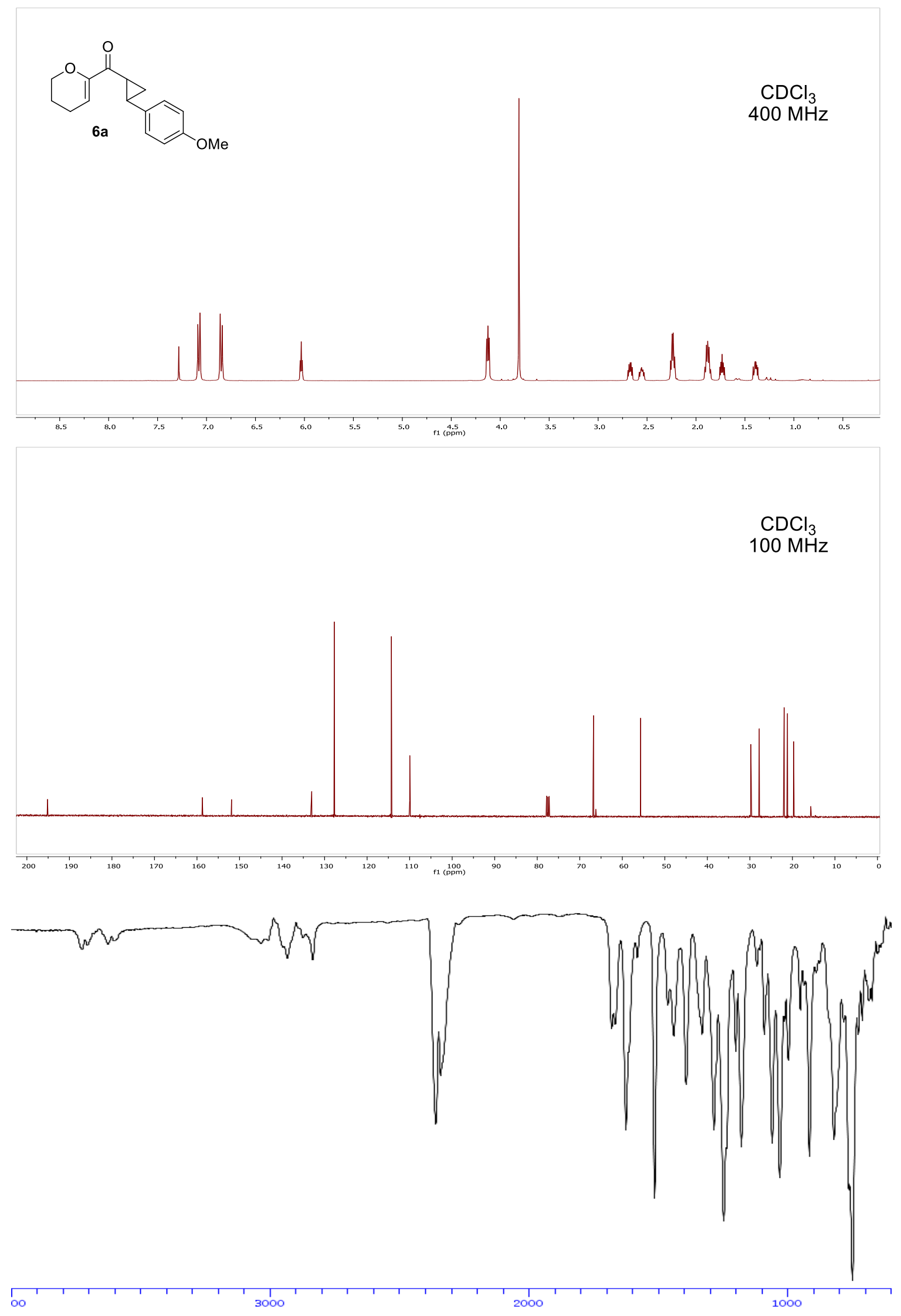

69 
solvent: $\angle \mathrm{CDCI} 3>$
Frequency. $400.13 \mathrm{MHz}$
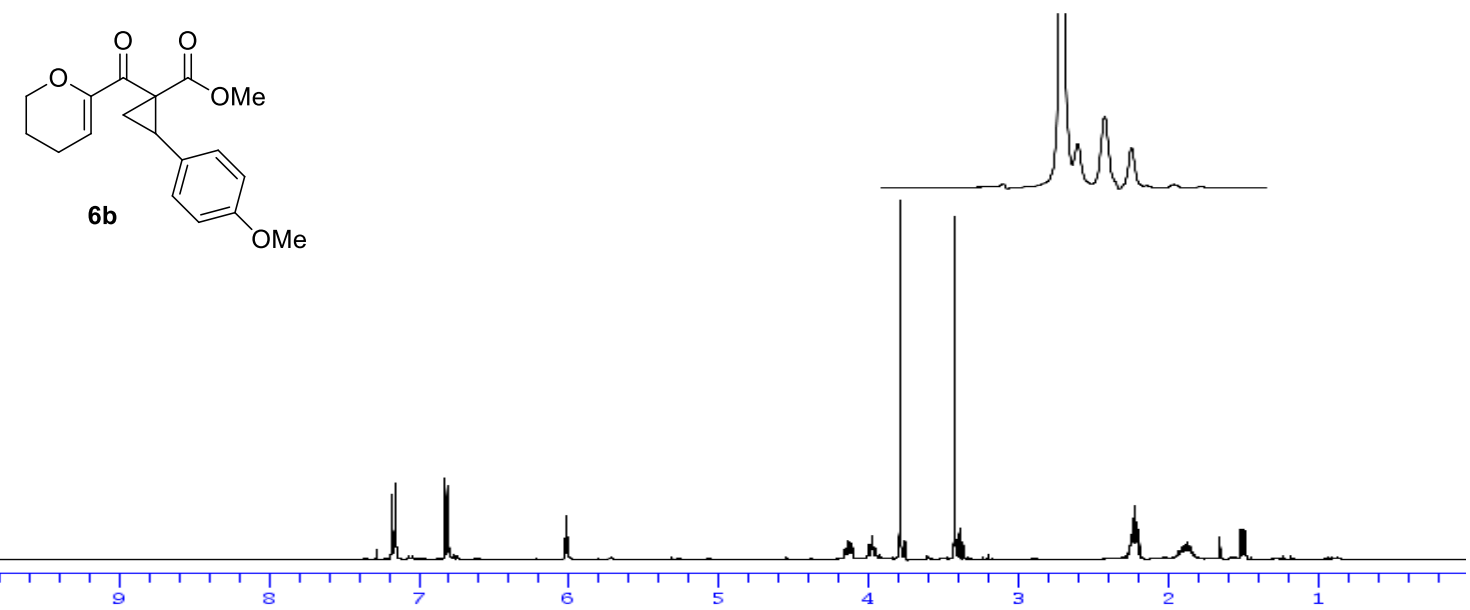

solvent: $<C D C I 3>$
Frequency. $100.612769 \mathrm{MHz}$
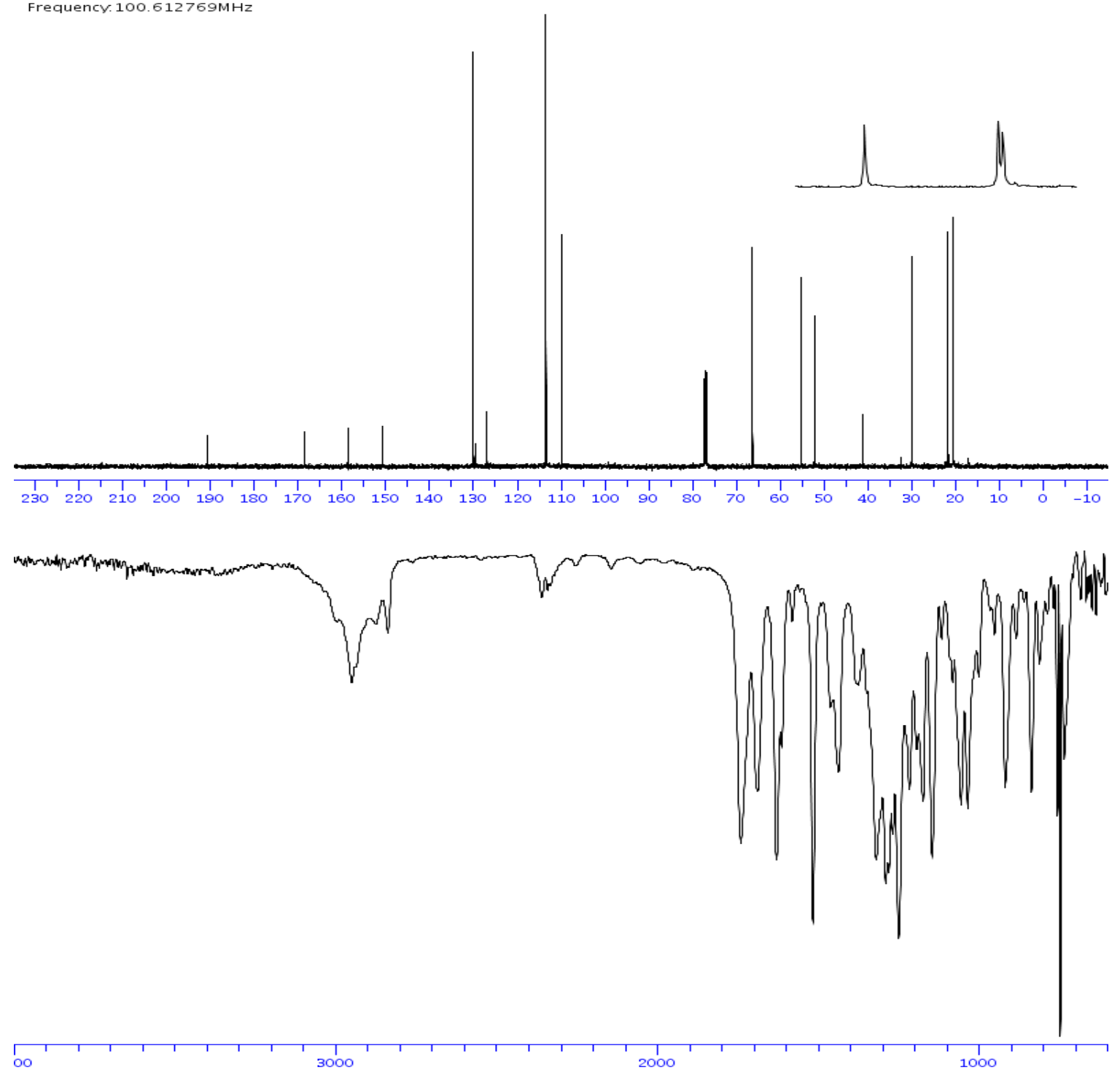

70 


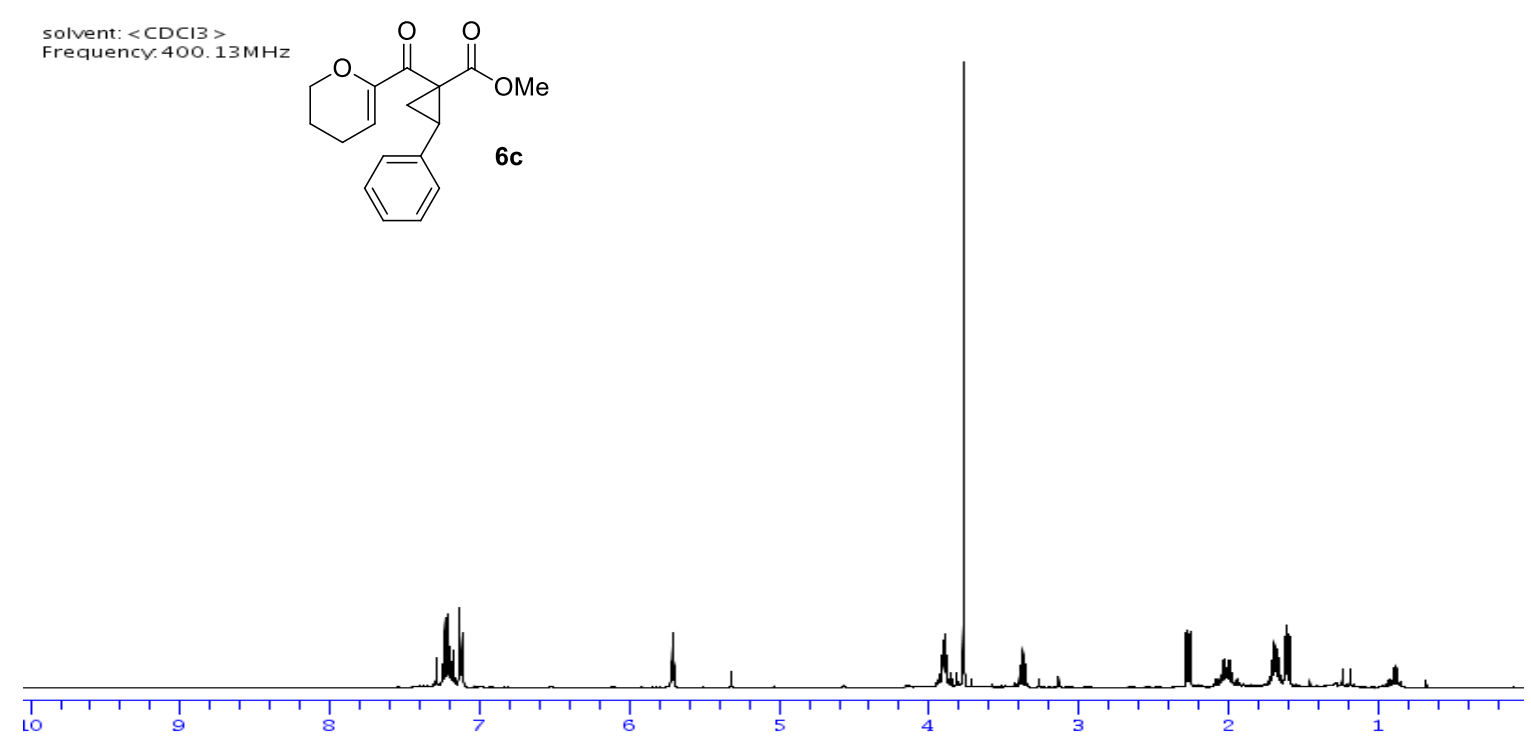

solvent: $<C D C 13>$

Frequency. 100.612769M Hz
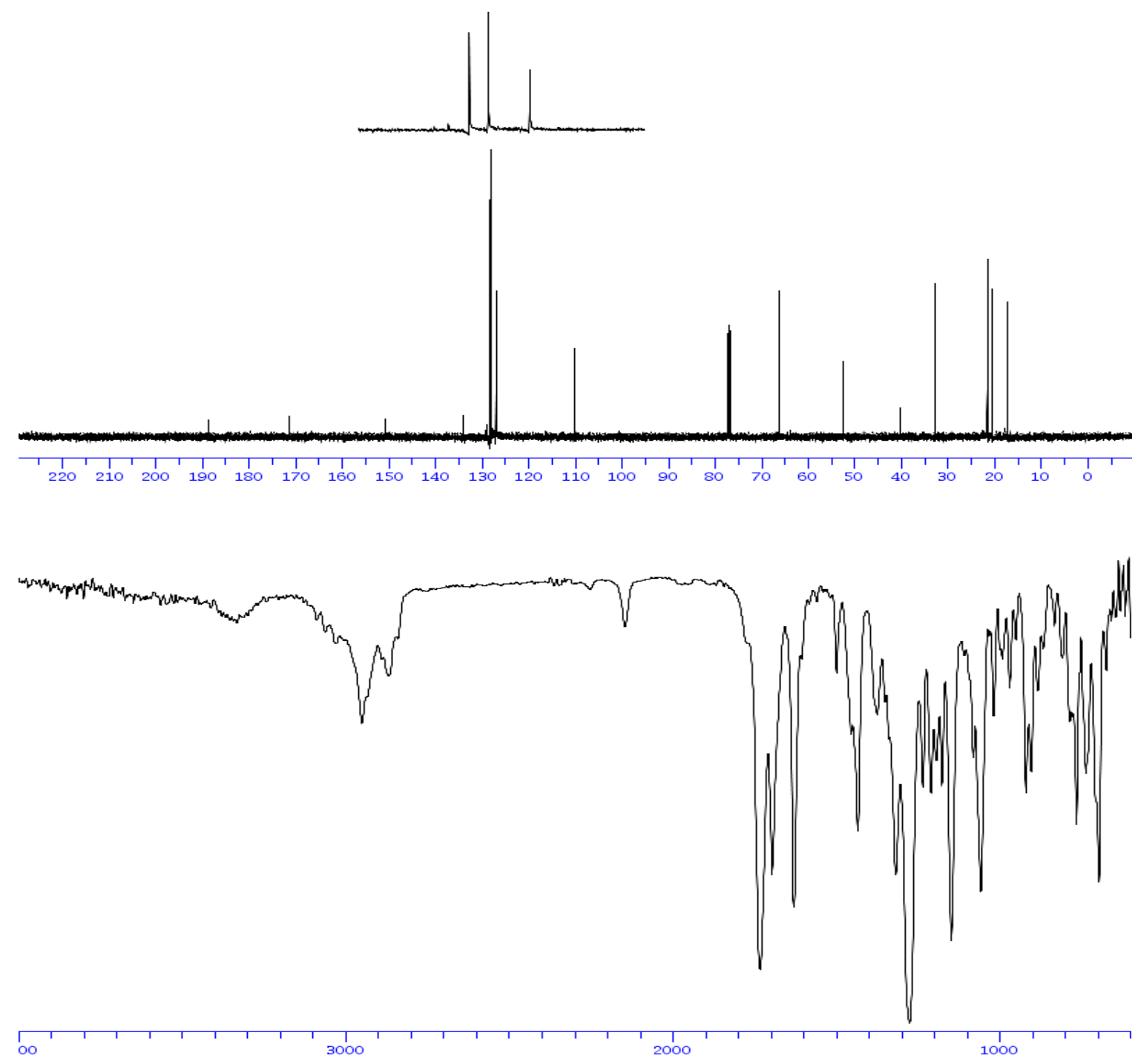


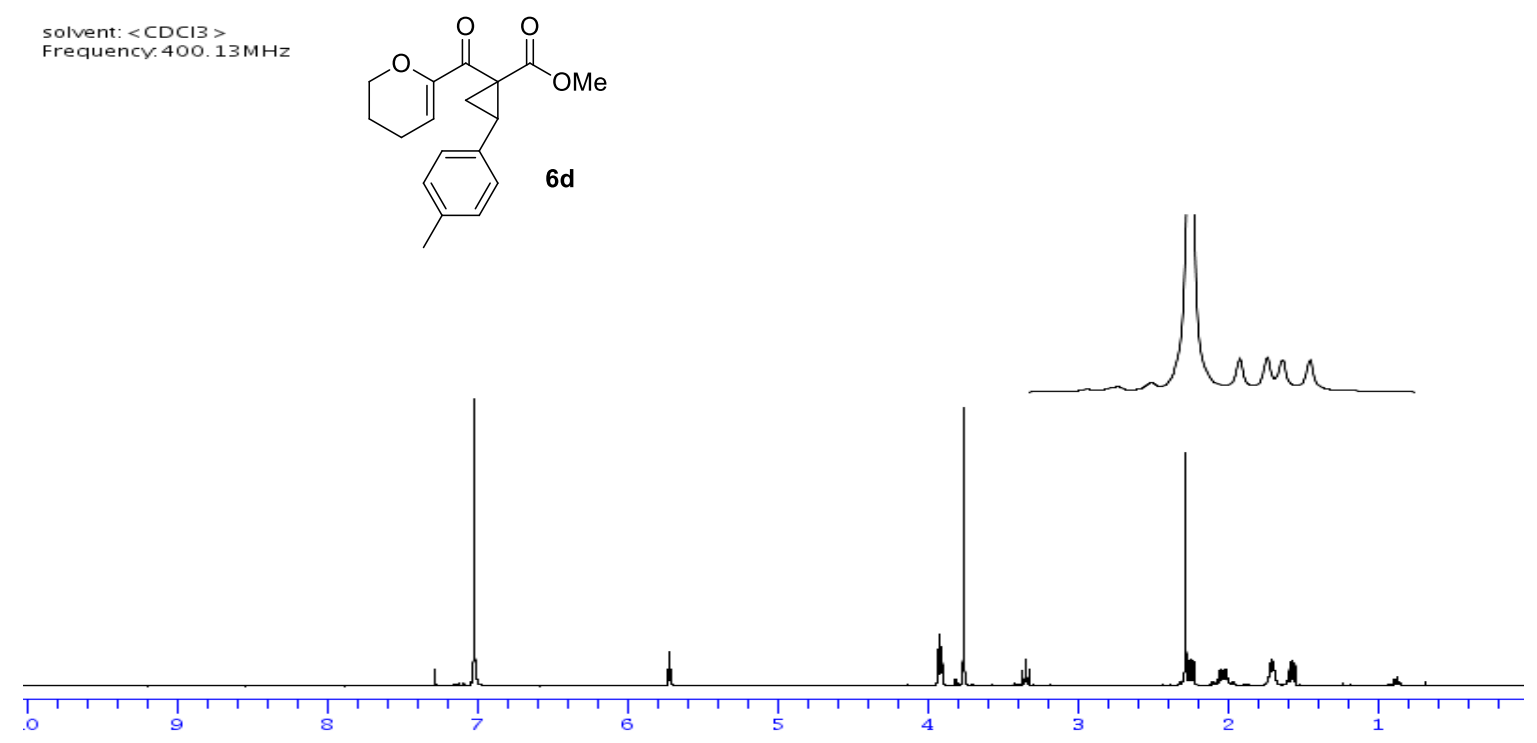

solvent: $<\mathrm{CDCl} 3>$
Frequency. $100.612769 \mathrm{MHz}$
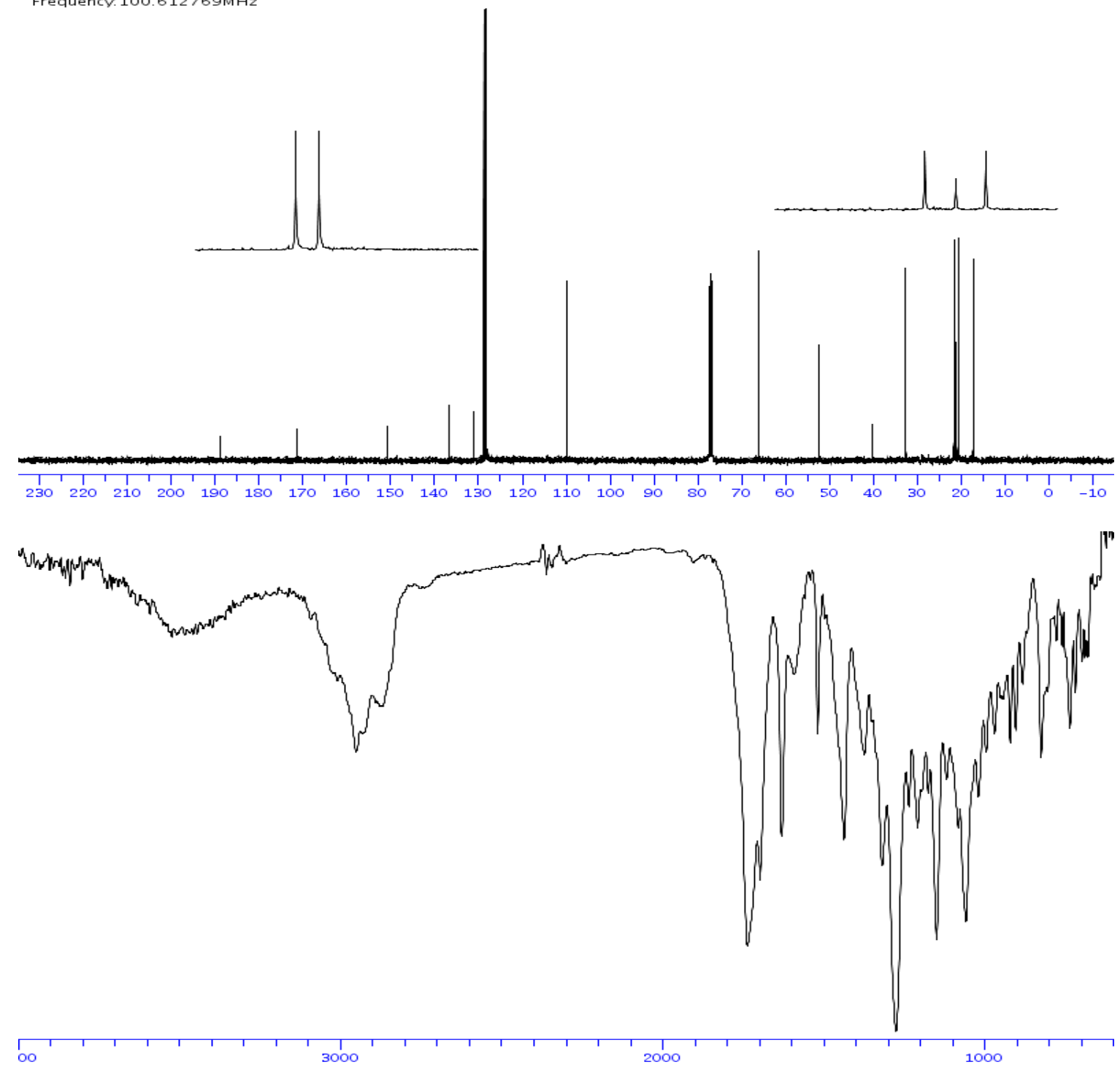
Solvent: $<\mathrm{CDCl} 3>$
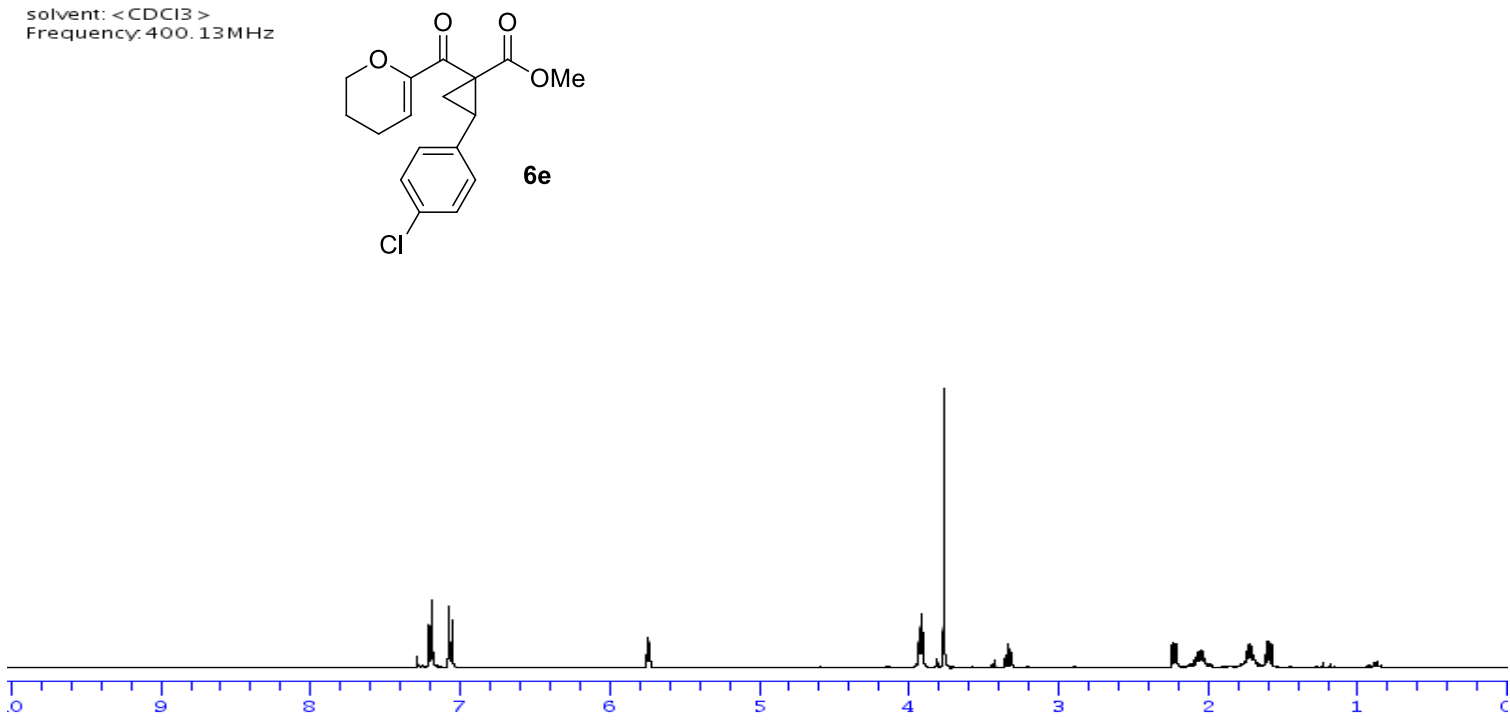

solvent: $\langle\mathrm{CDCI} 3\rangle$

Frequency $100.612769 \mathrm{MHz}$
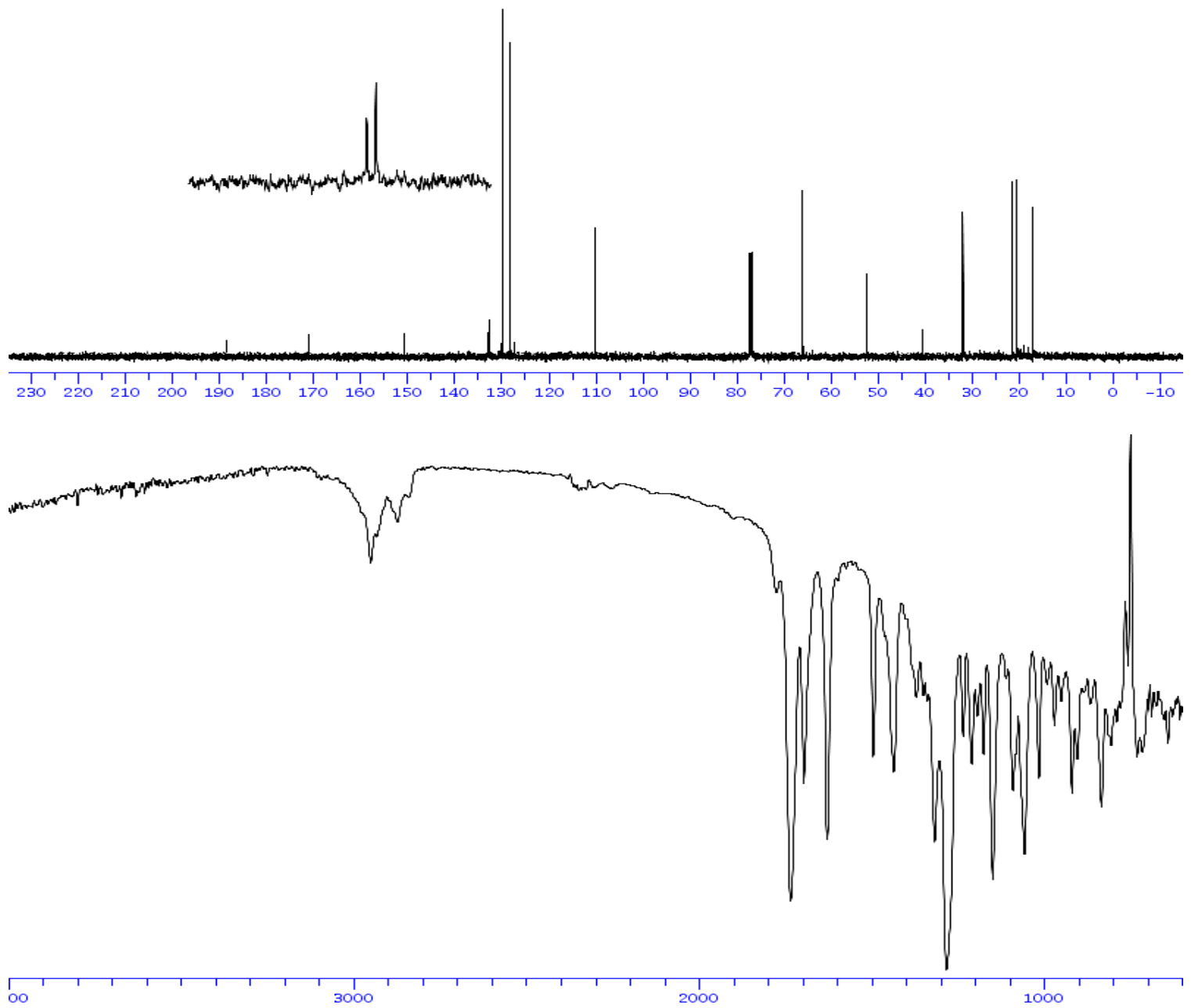
solvent: $\angle \mathrm{CDCI} 3>$
Frequency. $400.13 \mathrm{MHz}$
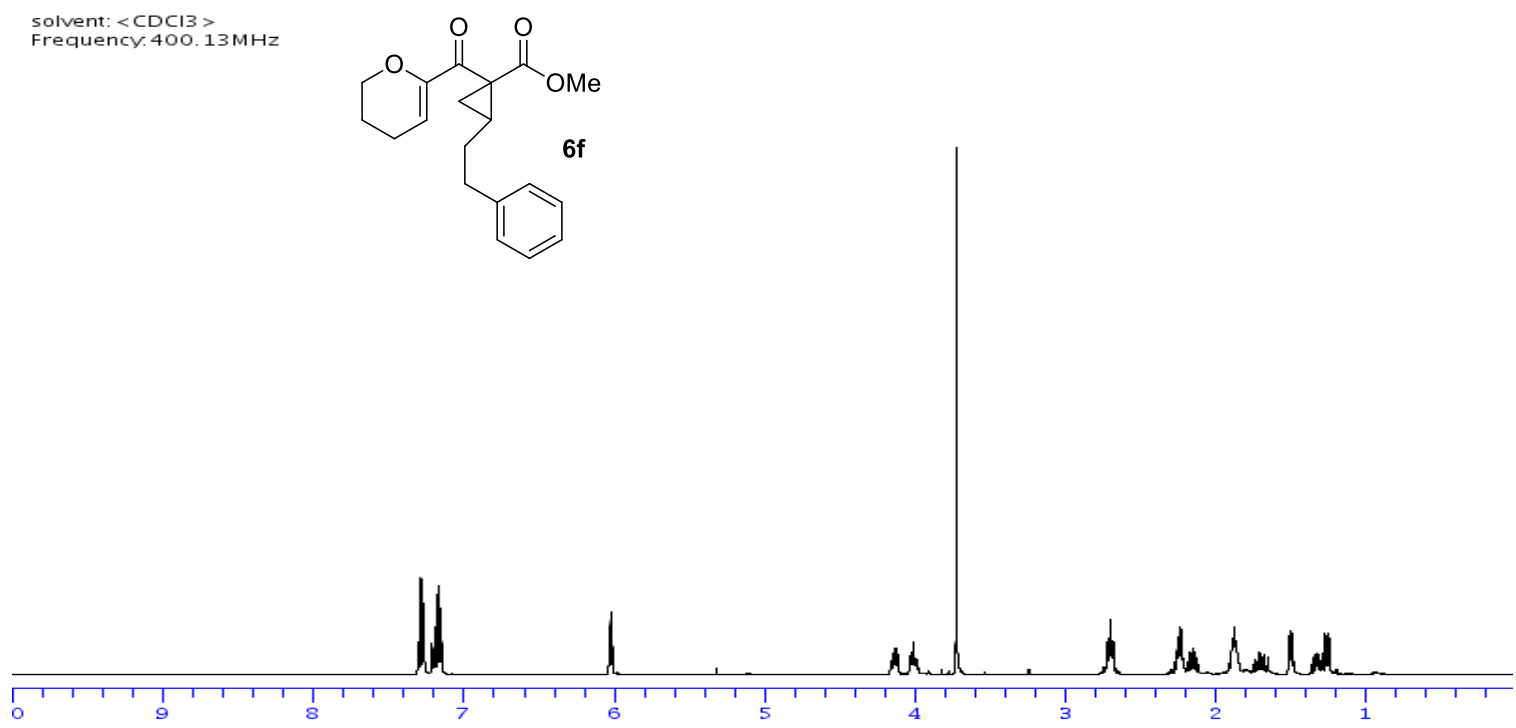

Solvent: $\angle C D C I 3>$
Frequency. $100.612769 \mathrm{MHz}$
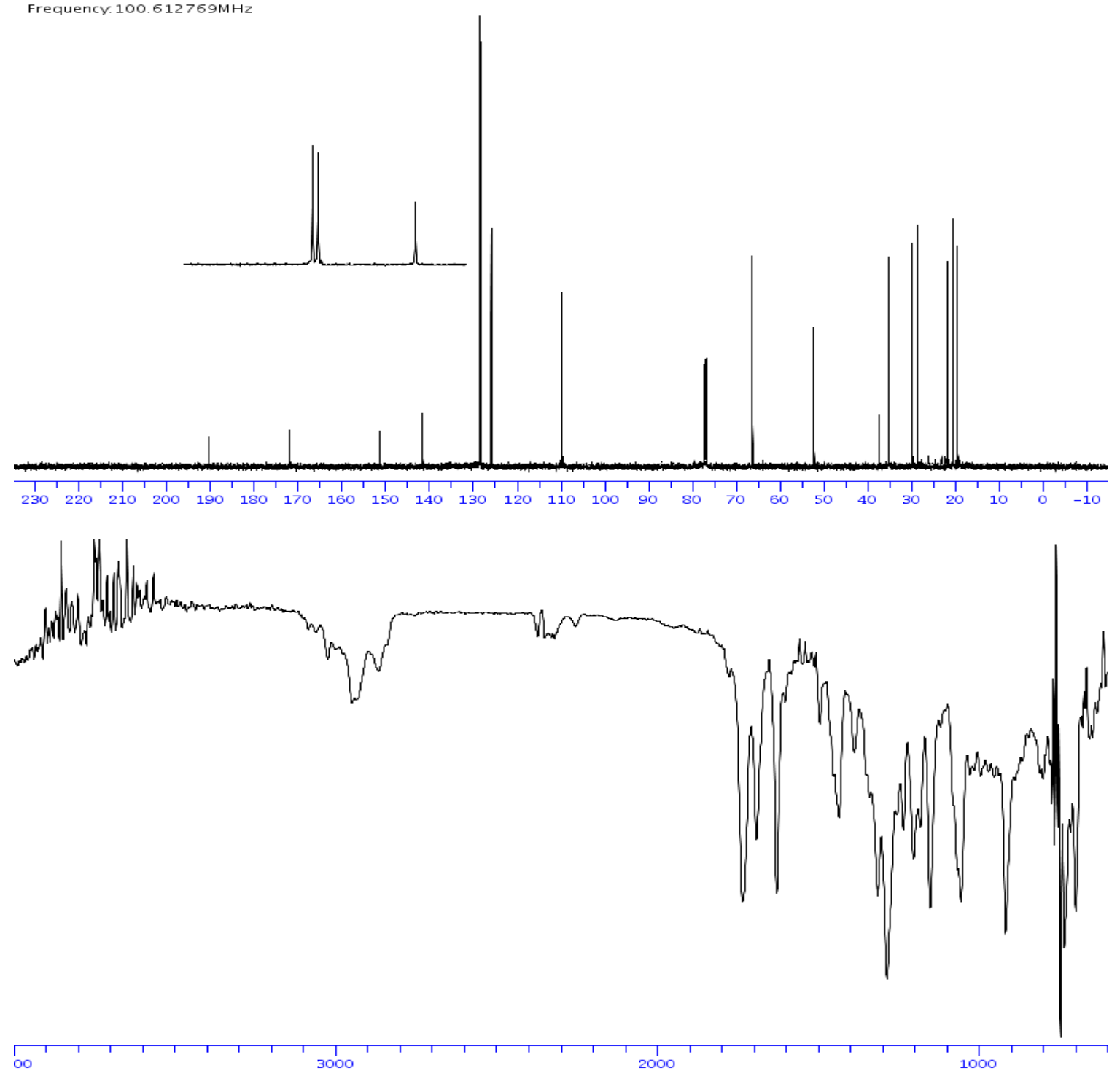

74 

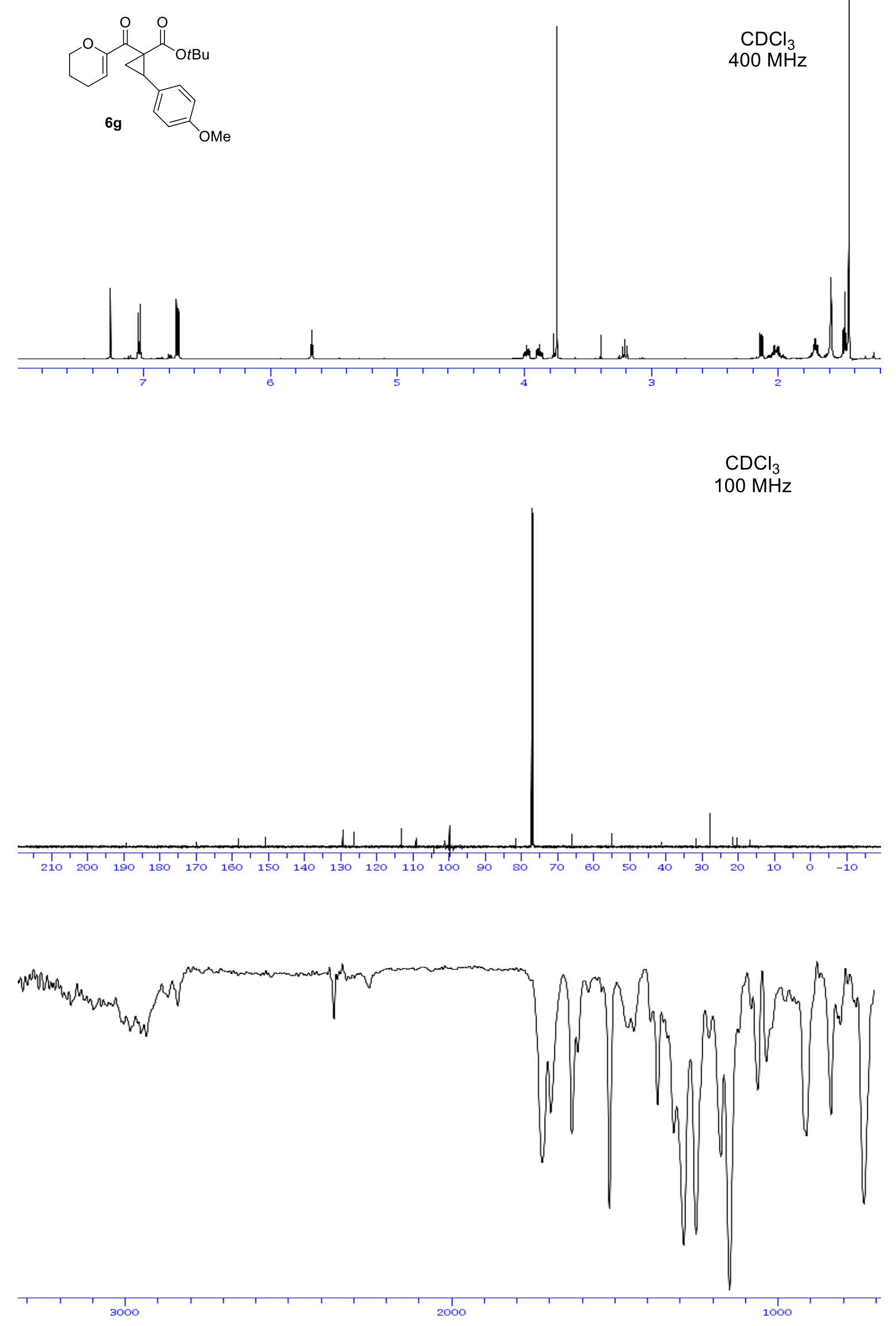

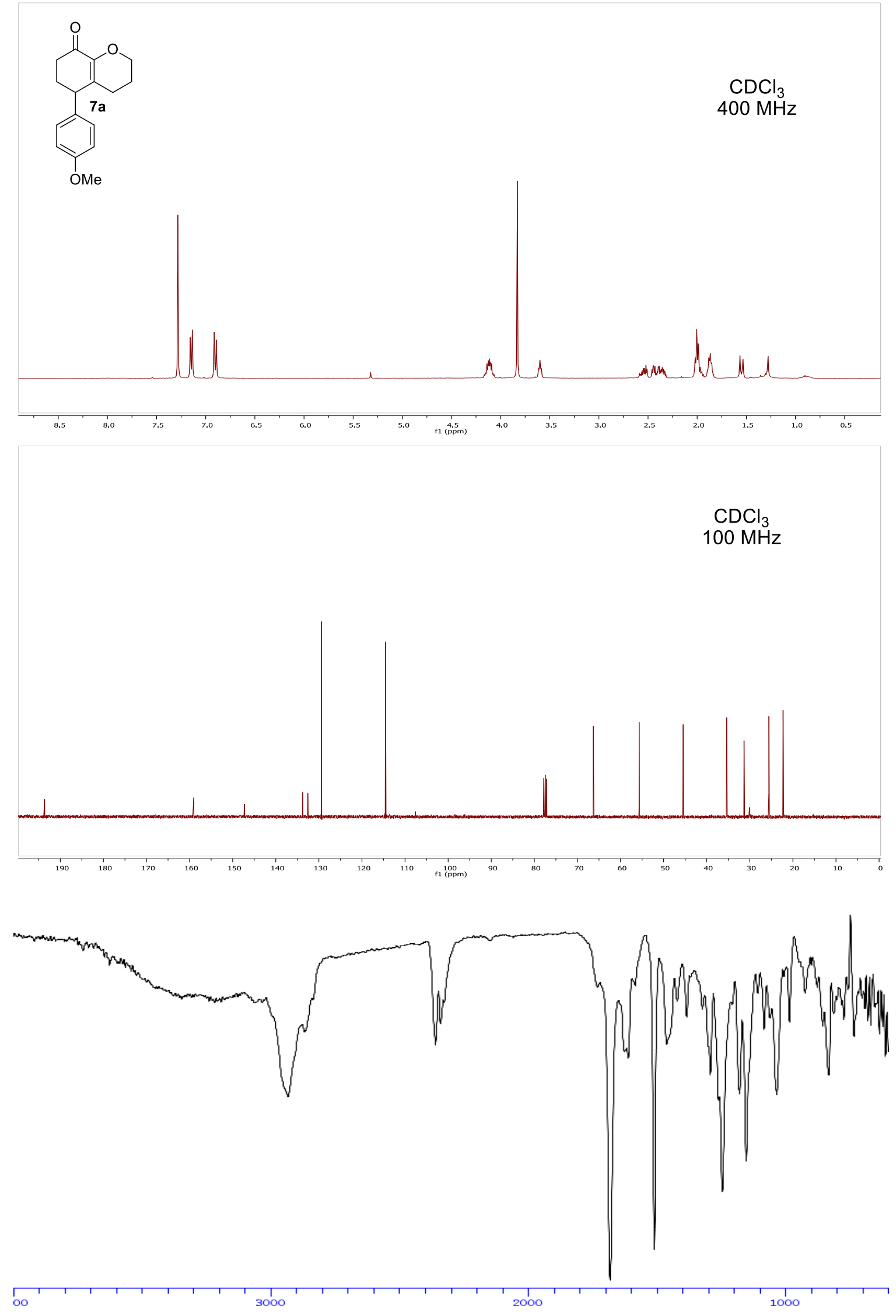
solvent: $<\mathrm{CDCI} 3>$
Frequency. $400.13 \mathrm{MHz}$

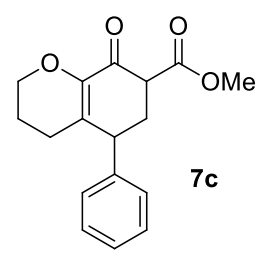

c

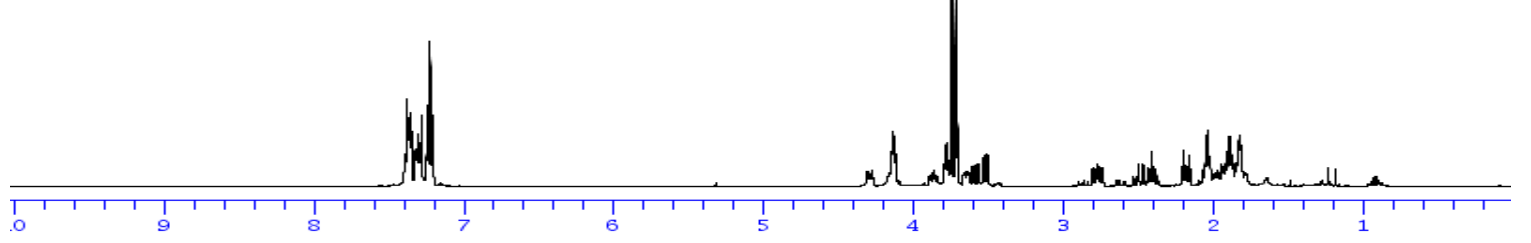

solvent: $\angle C D C I 3>$
Frequency. $400.13 \mathrm{MHz}$
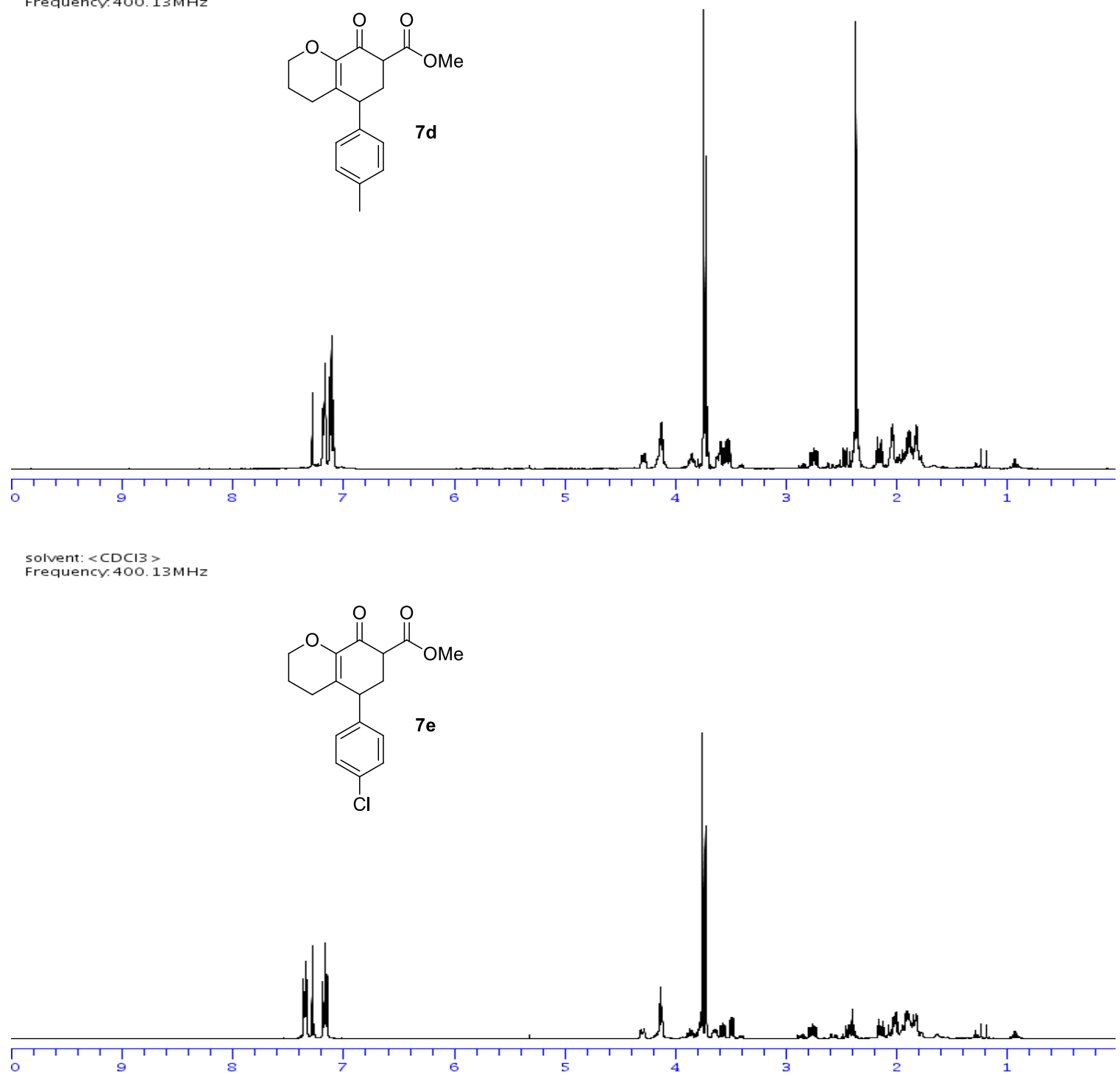

77 

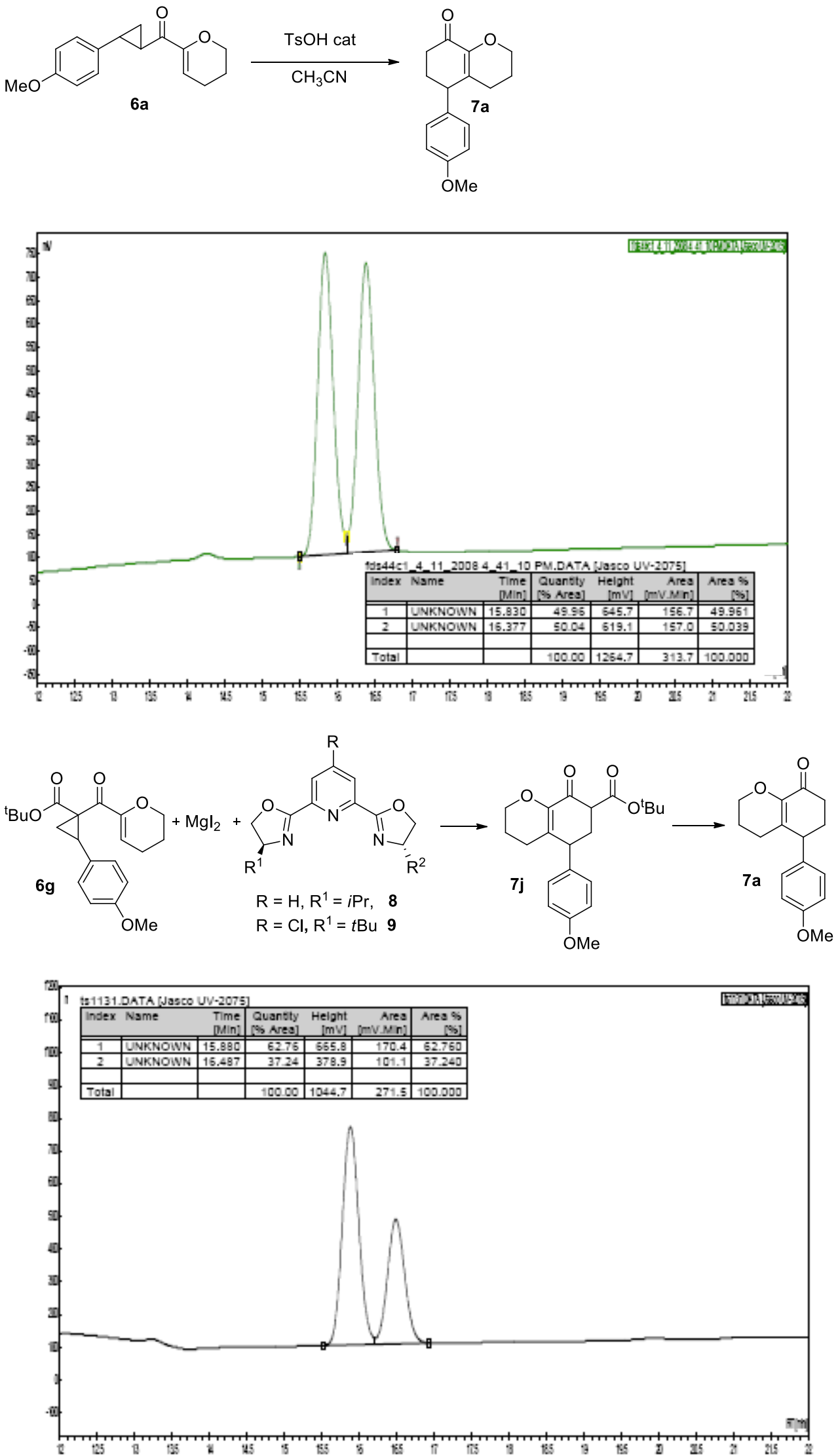

Acquisition time: $30 \mathrm{~min}$

Flow: $0.8 \mathrm{~mL} / \mathrm{min}$

Wavelength: $254 \mathrm{~nm}$

\begin{tabular}{|c|c|c|}
\hline Time $(\mathrm{min})$ & AcOEt $(\%)$ & Hexane (\%) \\
\hline 0 & 30 & 70 \\
\hline 8 & 50 & 50 \\
\hline 20 & 60 & 40 \\
\hline 30 & 100 & 0 \\
\hline
\end{tabular}


Solvent: $<\mathrm{CDCl} 3>$
Frequency, 400 . 13 $\mathrm{MHz}$<smiles>O=C1CCC(c2ccccc2)C2=C1OCCC2</smiles>

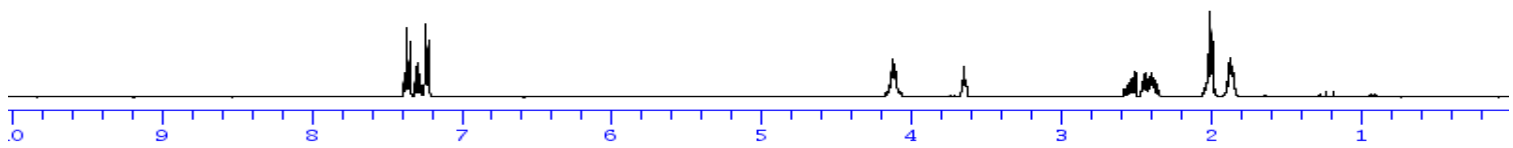

solvent: $\langle\mathrm{CDCI} 3\rangle$

.
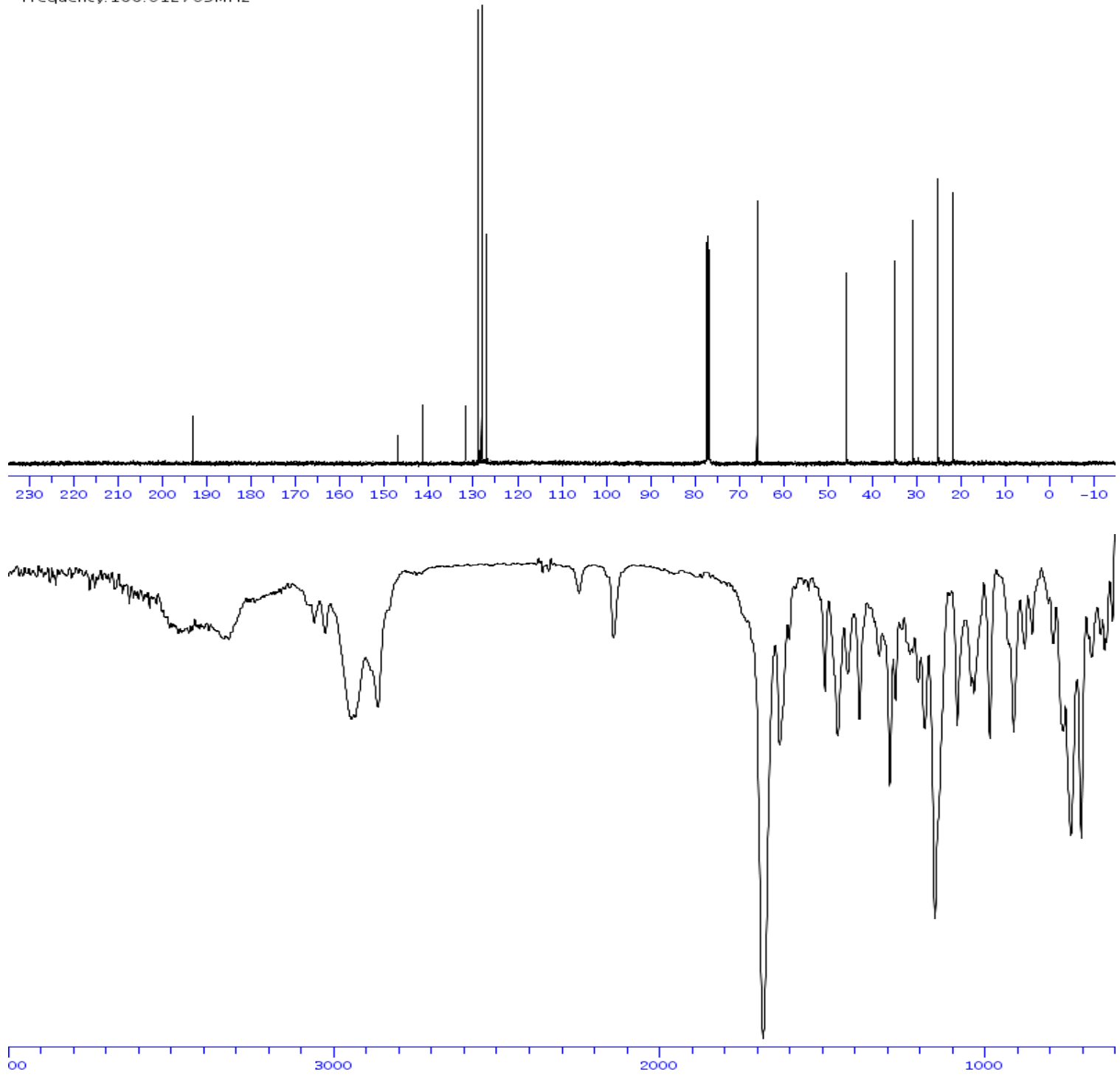
solvent: $\angle \mathrm{CDCl} 3\rangle$

$3 \mathrm{MHz}$
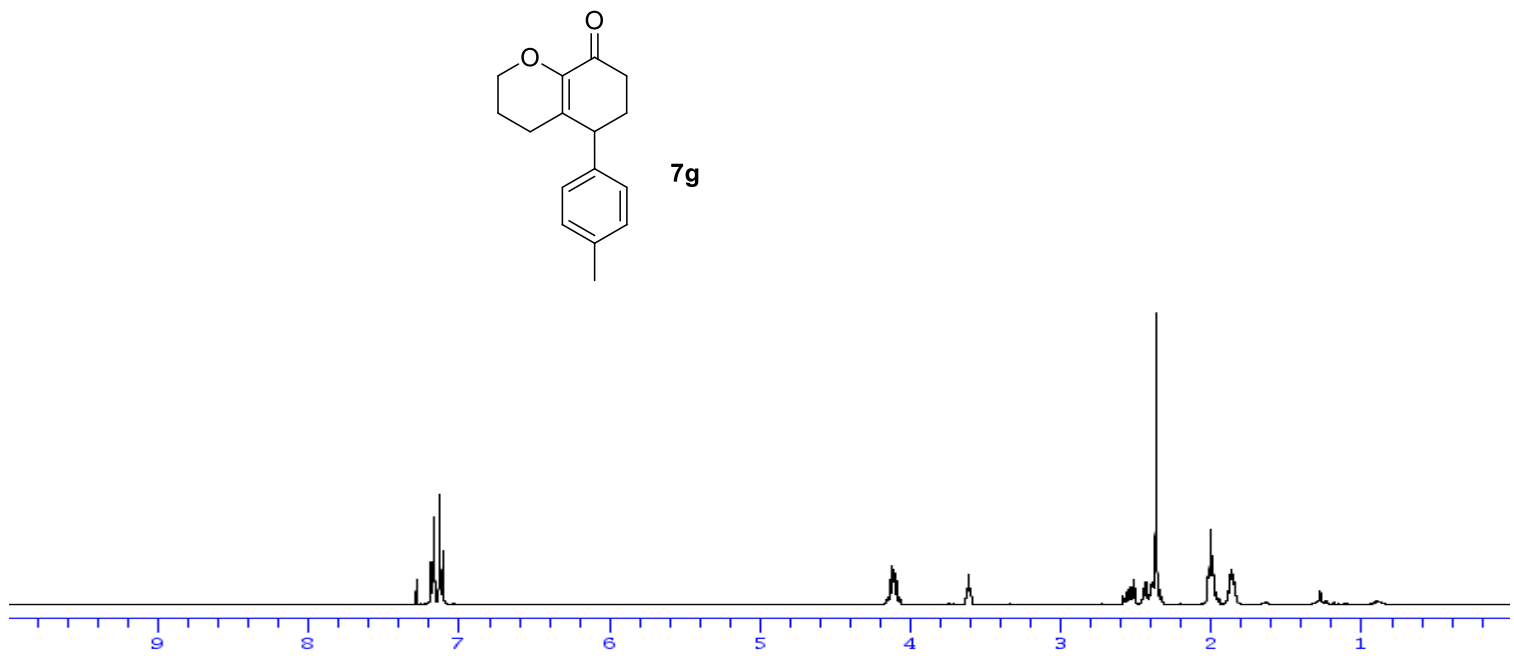

solvent: $<\mathrm{CDCI} 3>$

FrequencY $100.612769 \mathrm{MHz}$

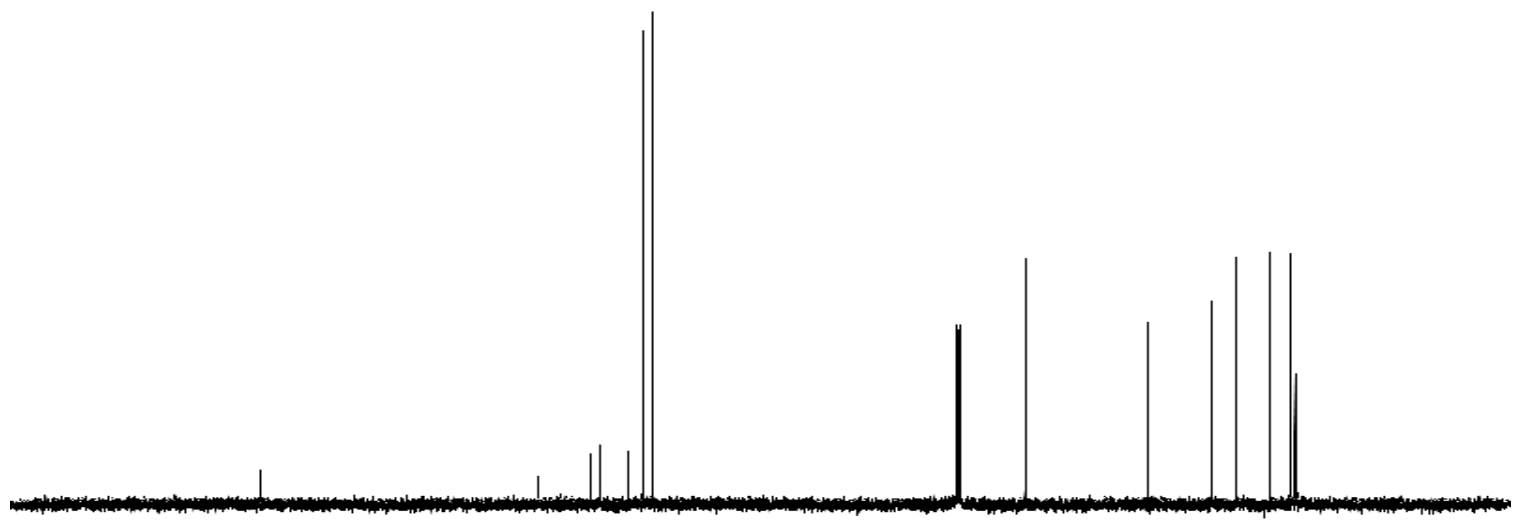

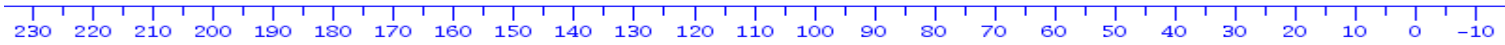

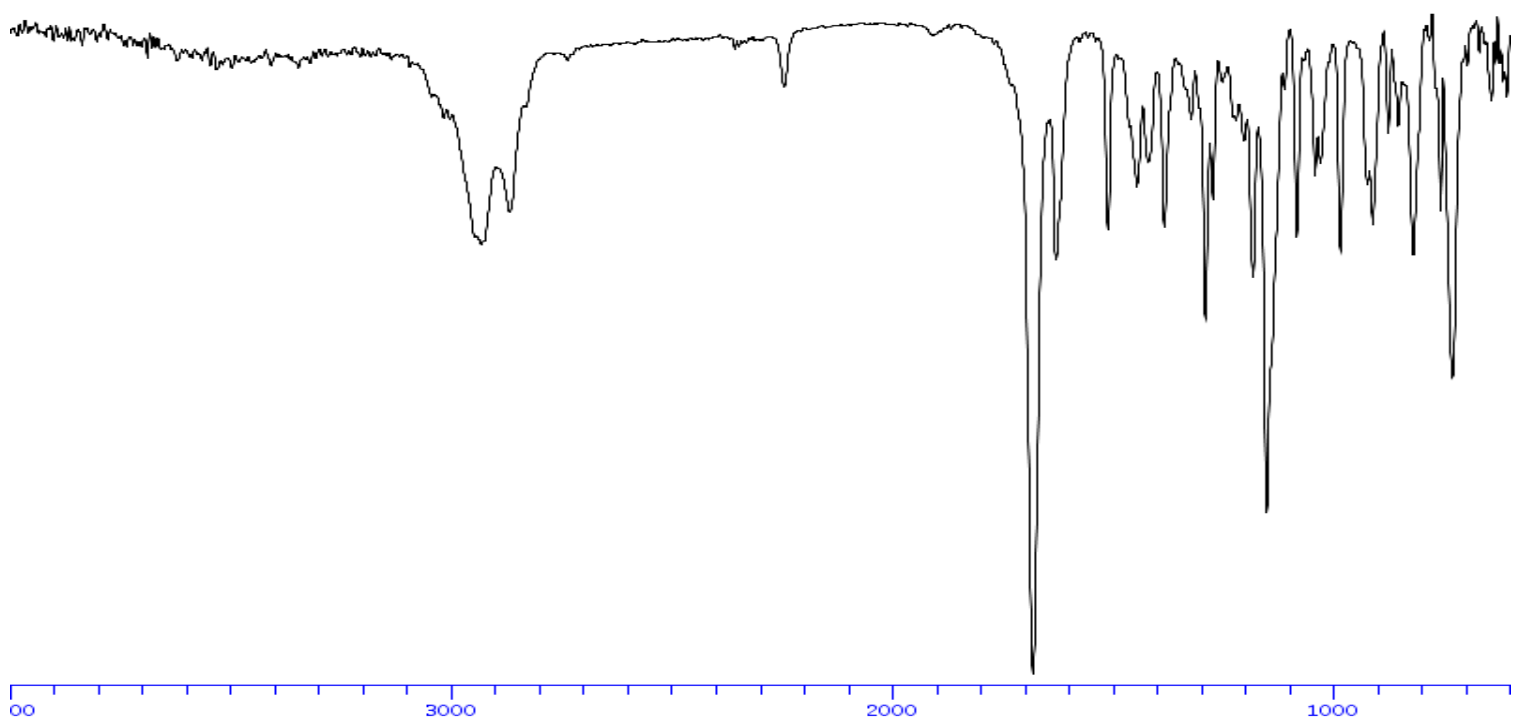


solvent: $\angle \mathrm{CDCI} 3\rangle$

OO. $13 \mathrm{MHz}$
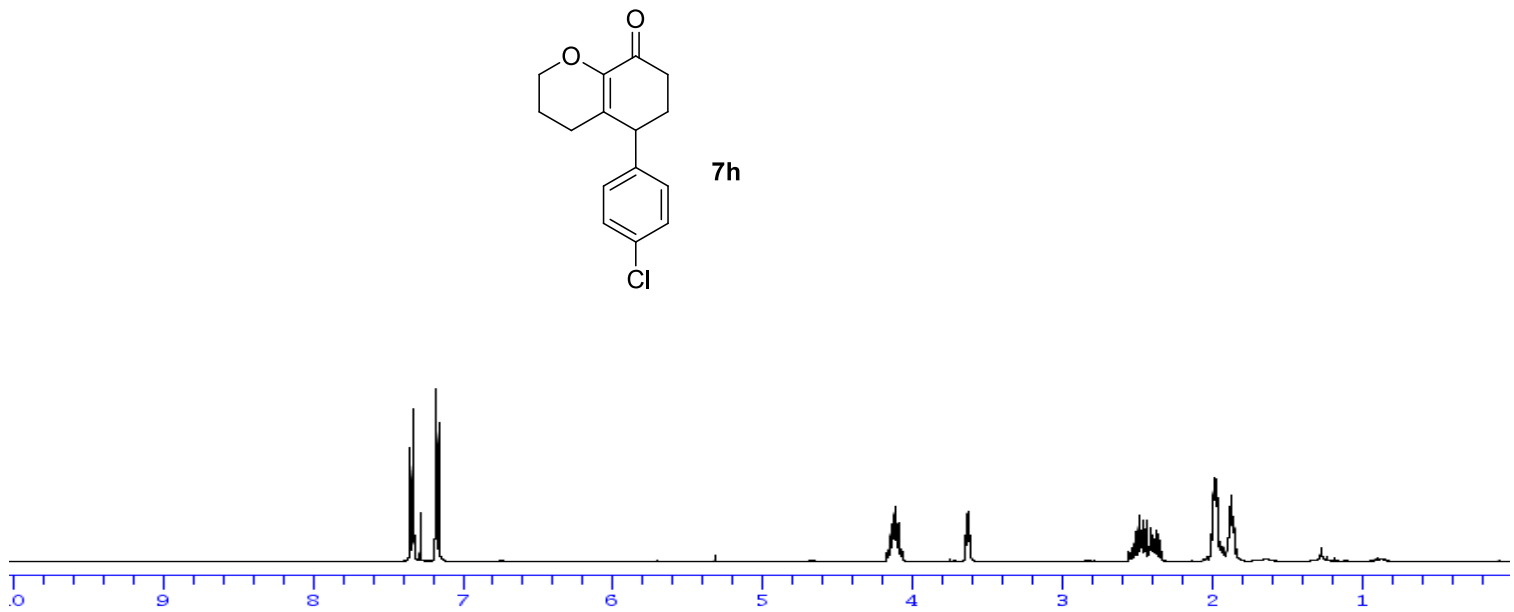

solvent: $\langle\mathrm{CDCI}\rangle$

$.612769 \mathrm{MHz}$

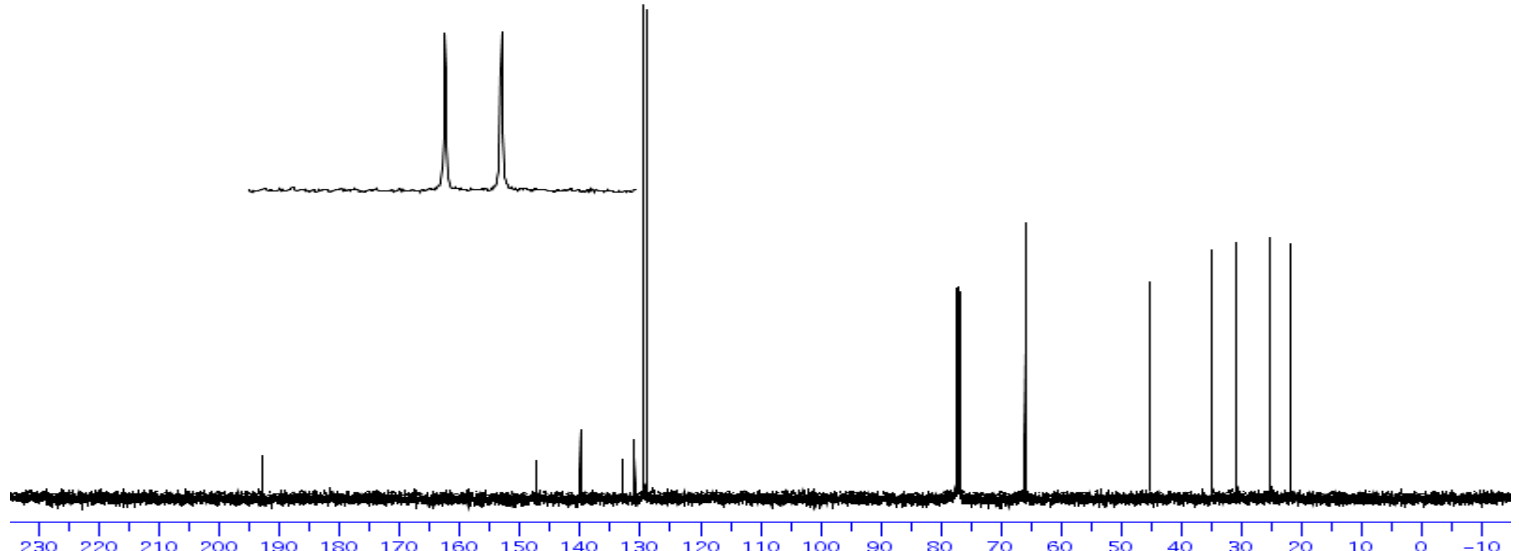

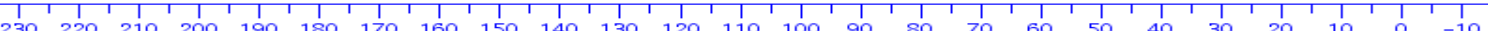

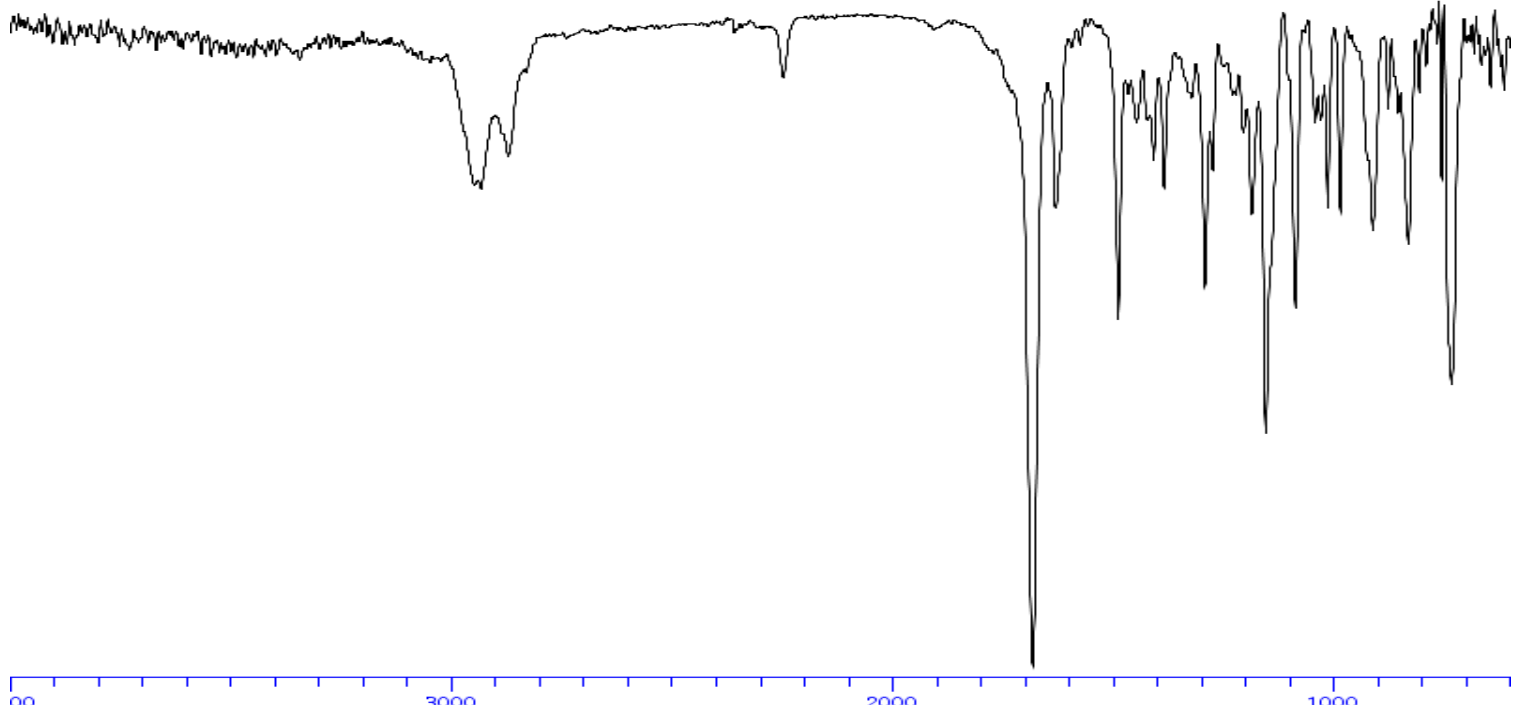



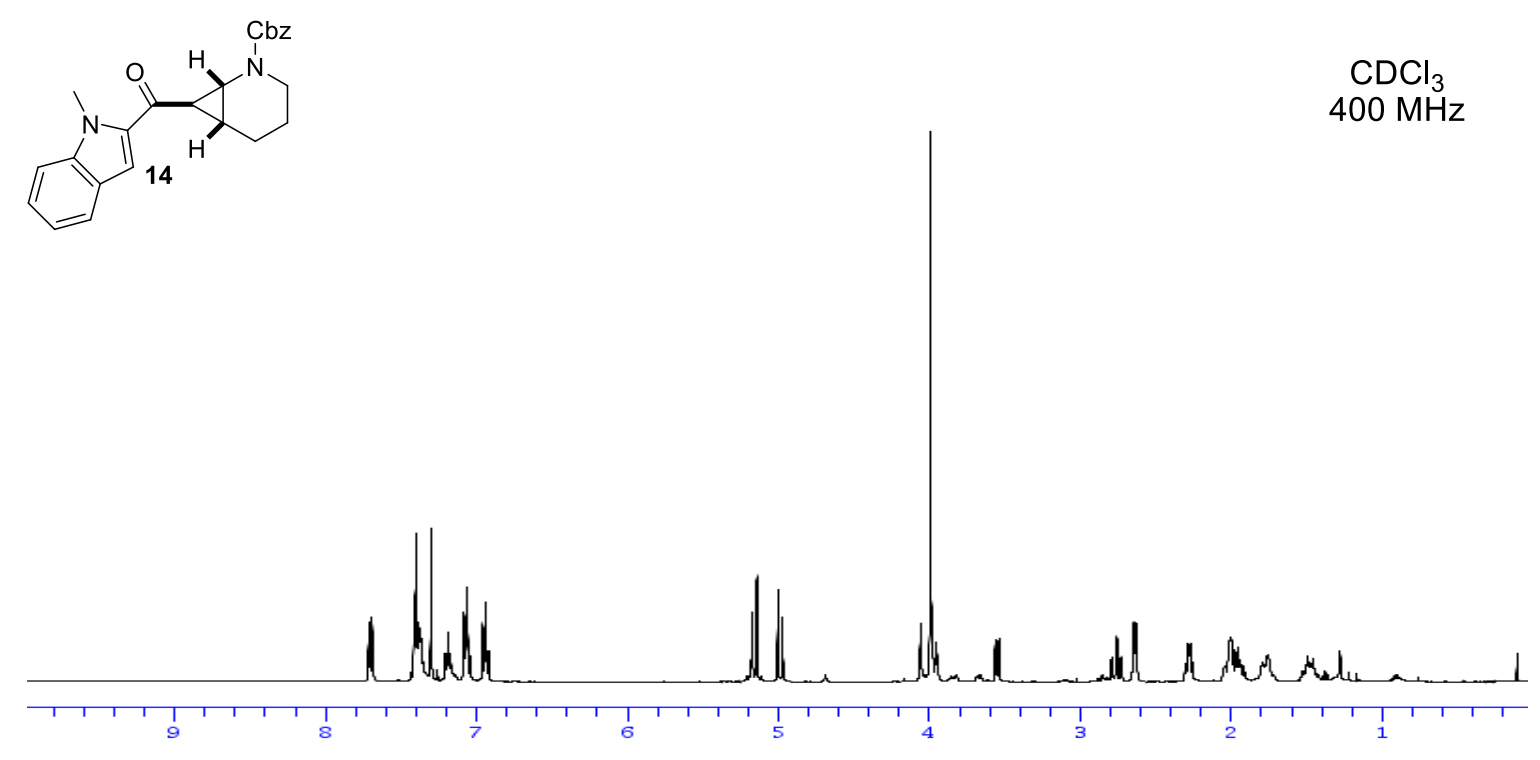

$\mathrm{CDCl}_{3}$

$100 \mathrm{MHz}$
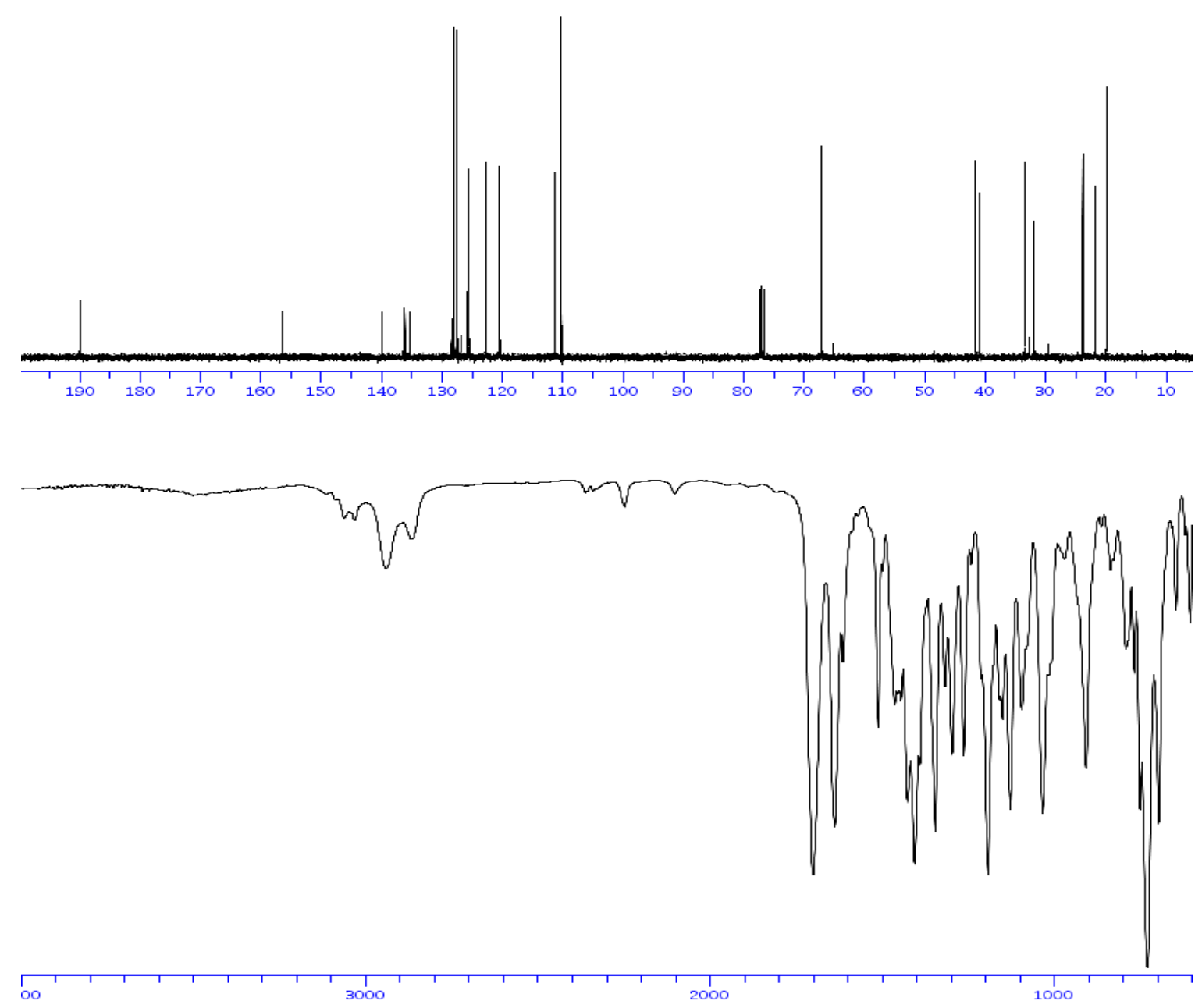


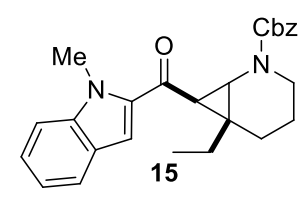

$\mathrm{CDCl}_{3}$

$400 \mathrm{MHz}$

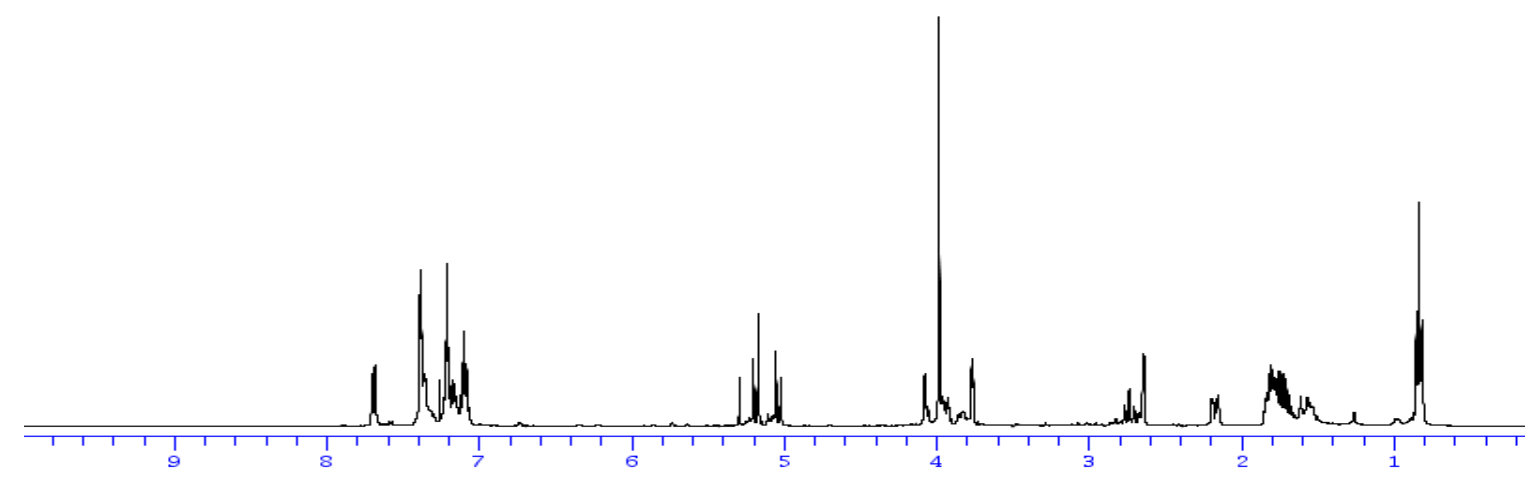

$\mathrm{CDCl}_{3}$

$100 \mathrm{MHz}$
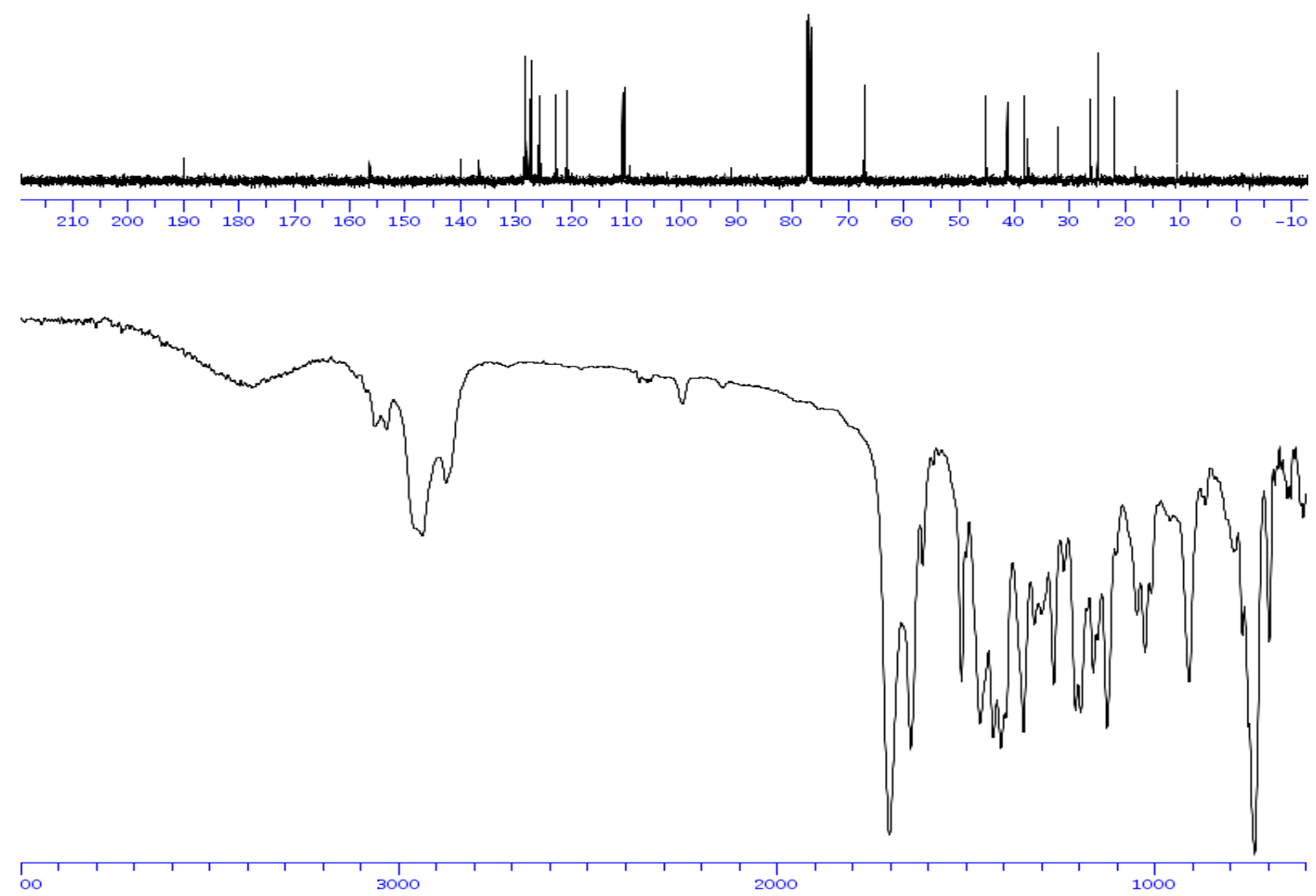


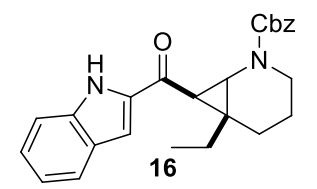

$\mathrm{CDCl}_{3}$

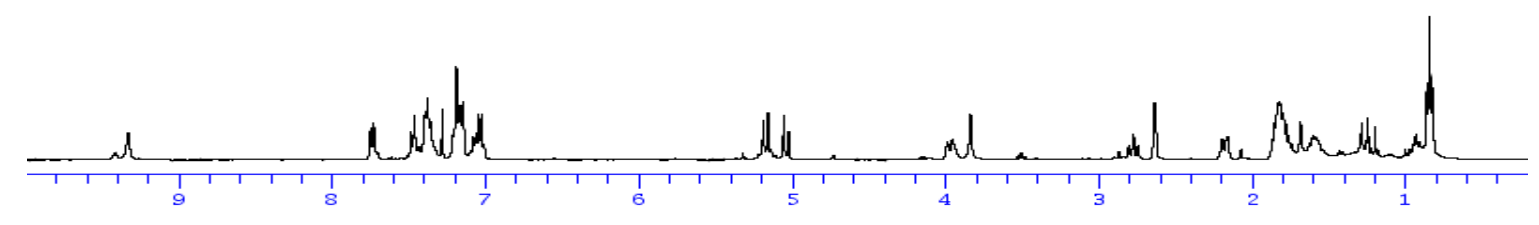

$\mathrm{CDCl}_{3}$

$100 \mathrm{MHz}$
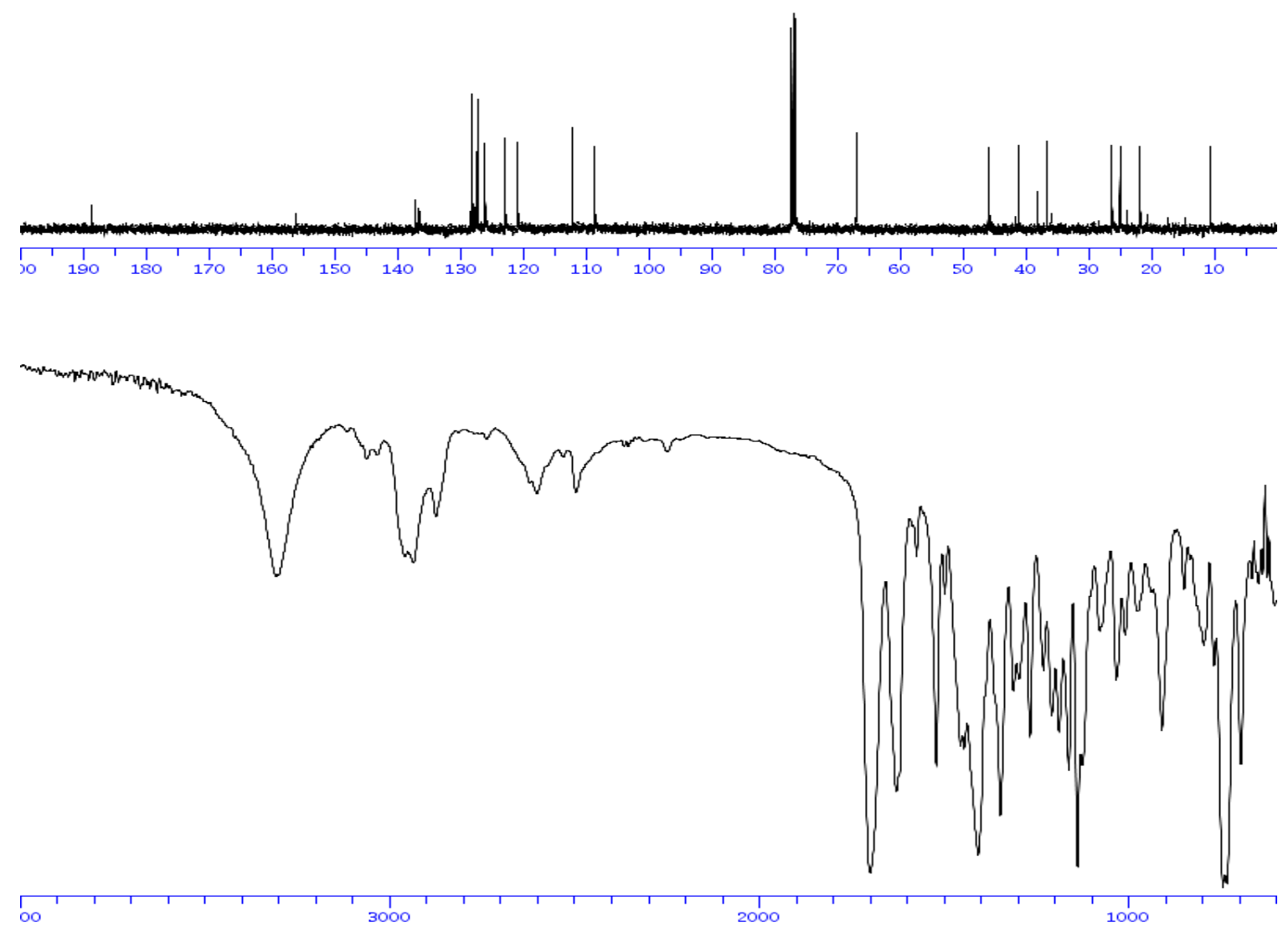


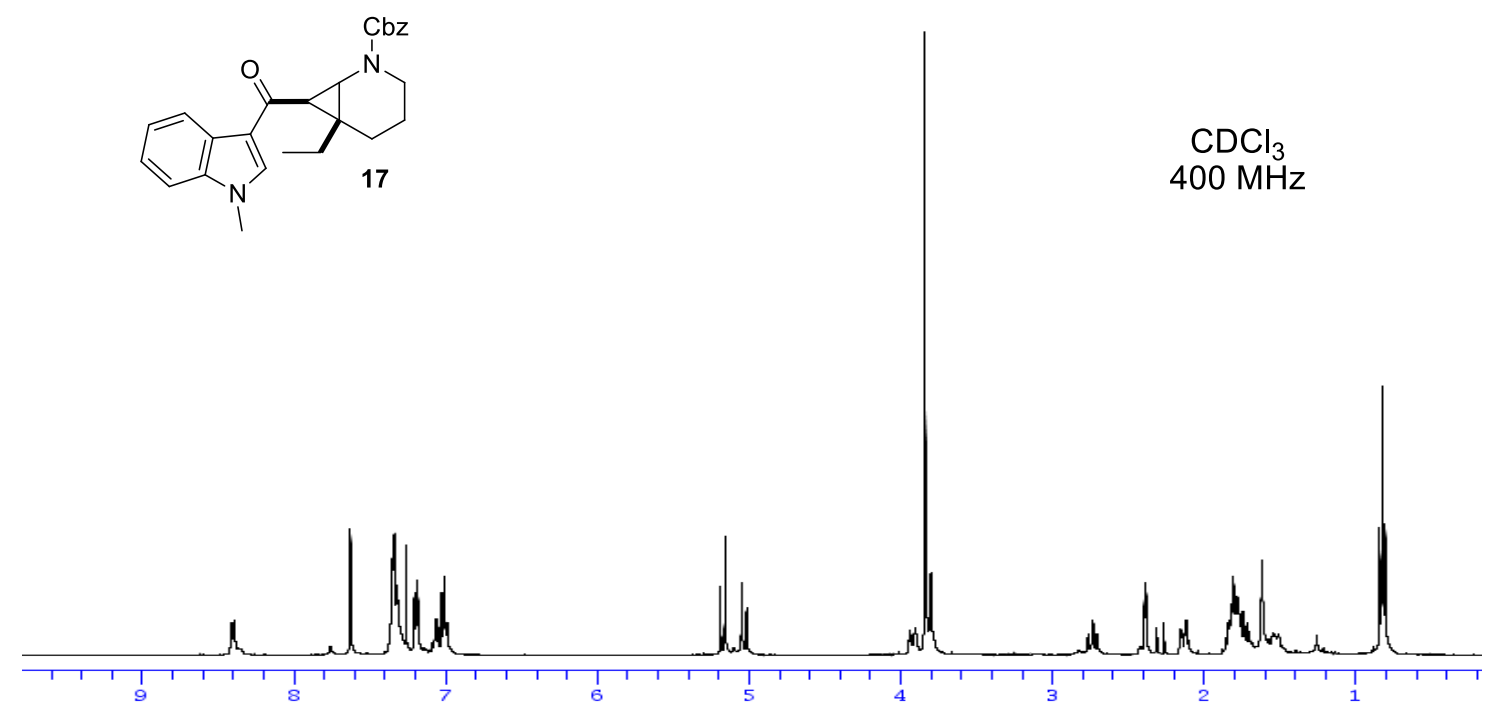

$\mathrm{CDCl}_{3}$

$100 \mathrm{MHz}$
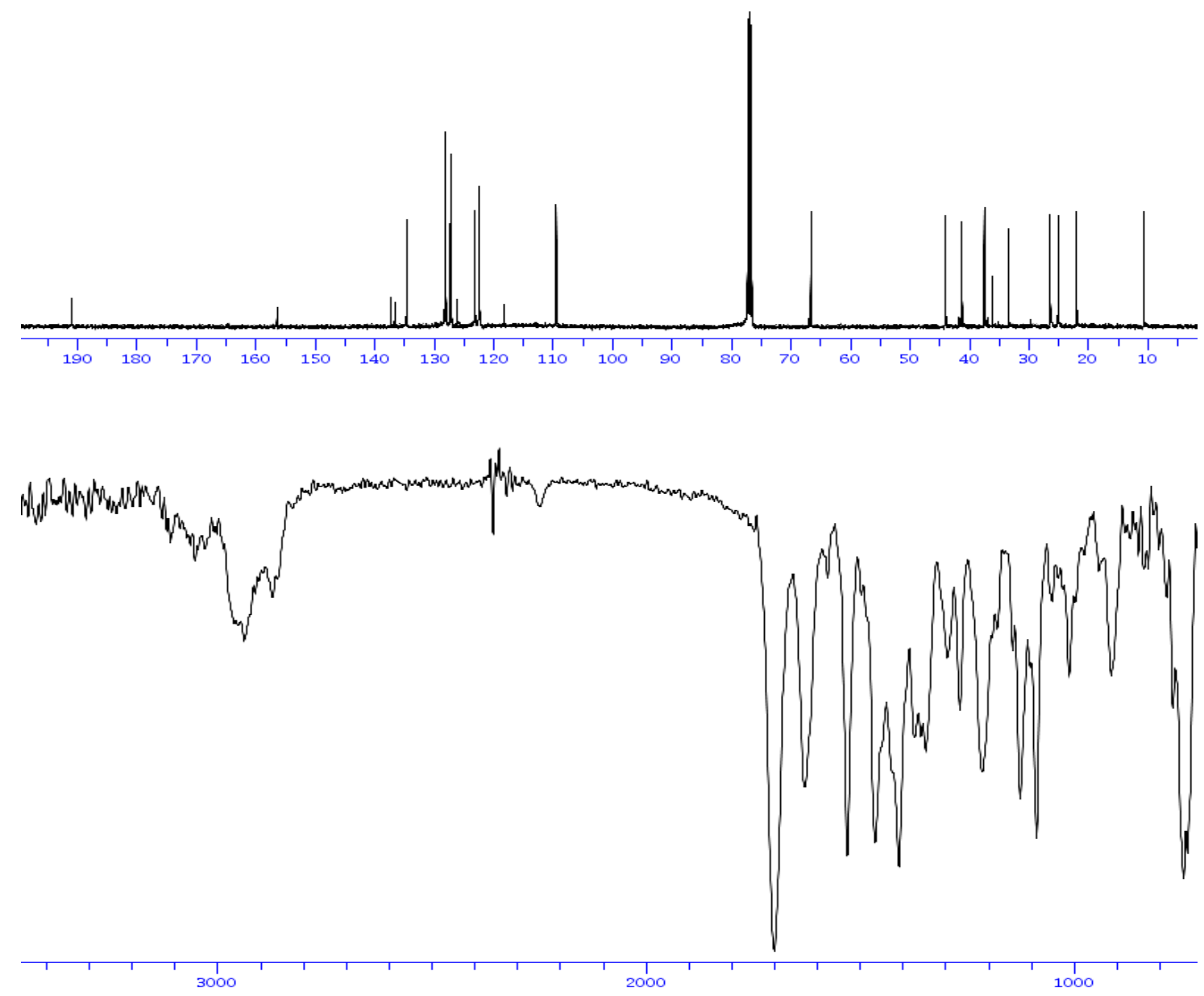


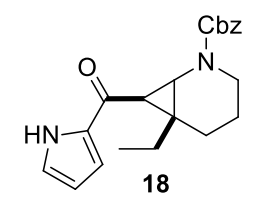

$\mathrm{CDCl}_{3}$

$400 \mathrm{MHz}$
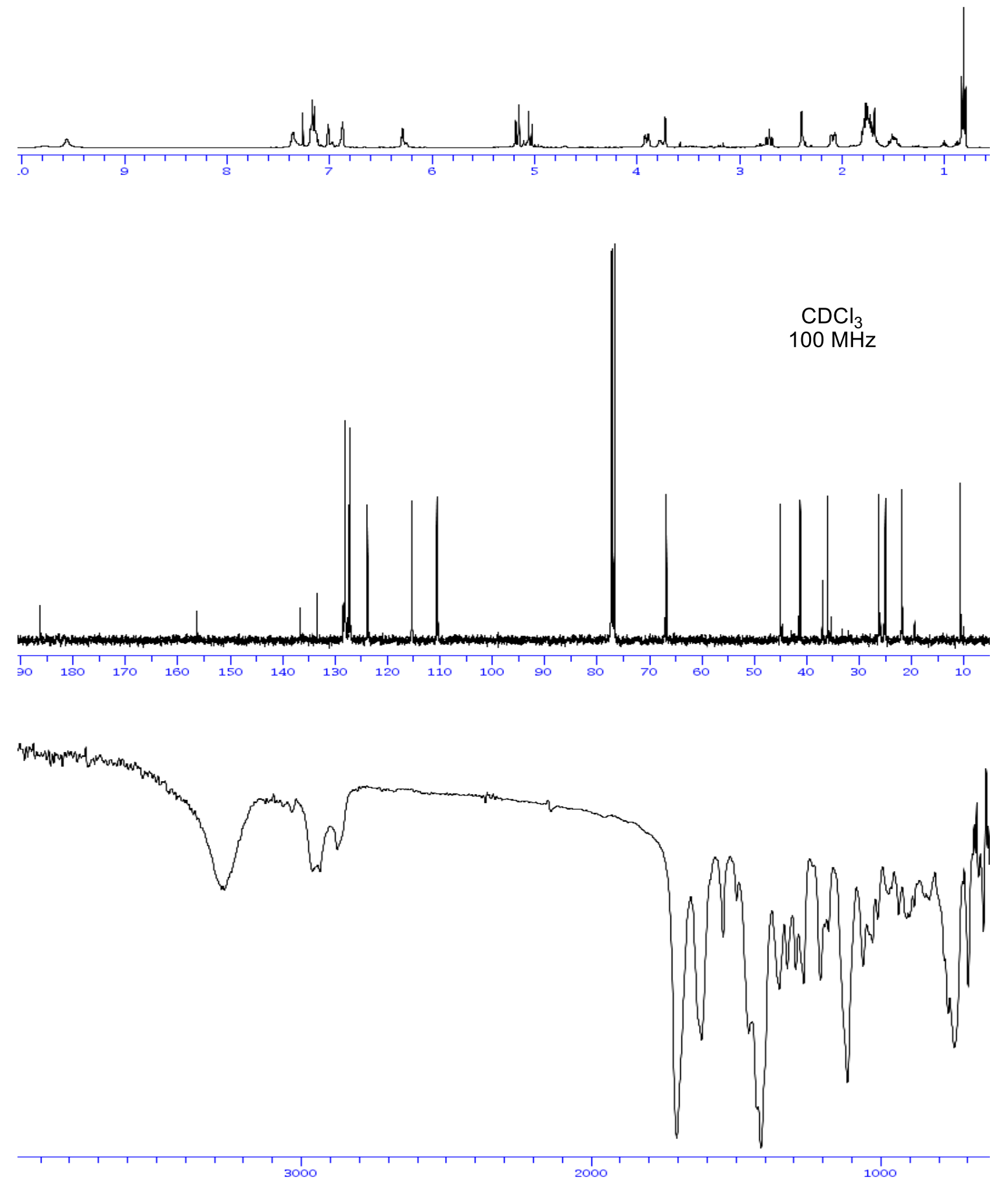


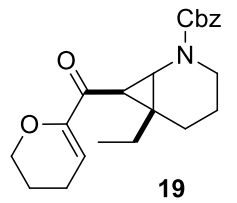

$\mathrm{CDCl}_{3}$

$400 \mathrm{MHz}$

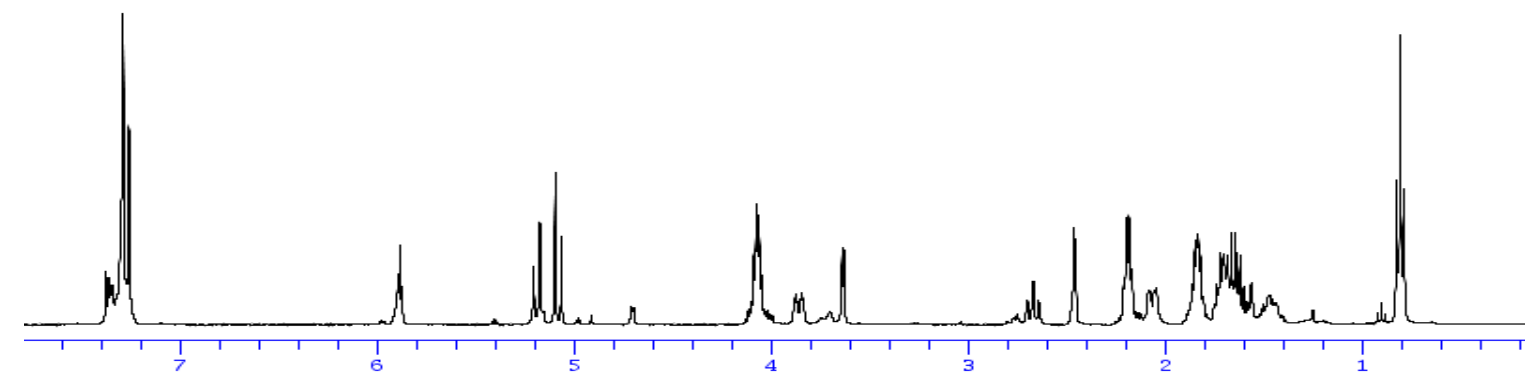

$\mathrm{CDCl}_{3}$

$100 \mathrm{MHz}$
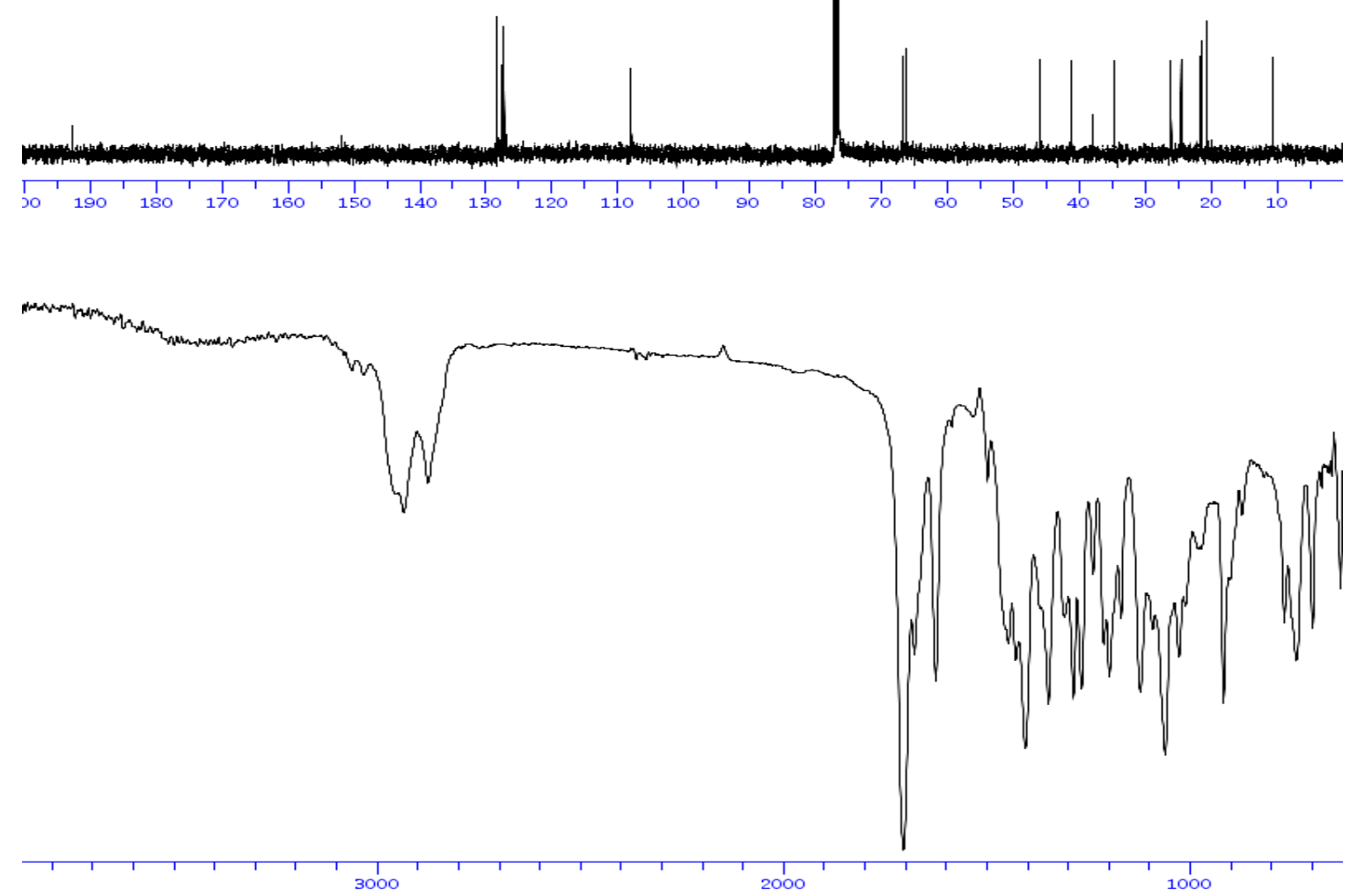
<smiles></smiles>

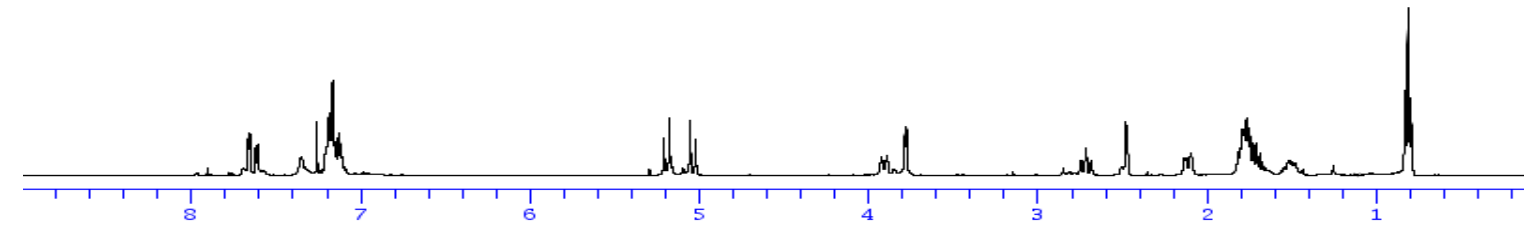

$\mathrm{CDCl}_{3}$

$100 \mathrm{MHz}$
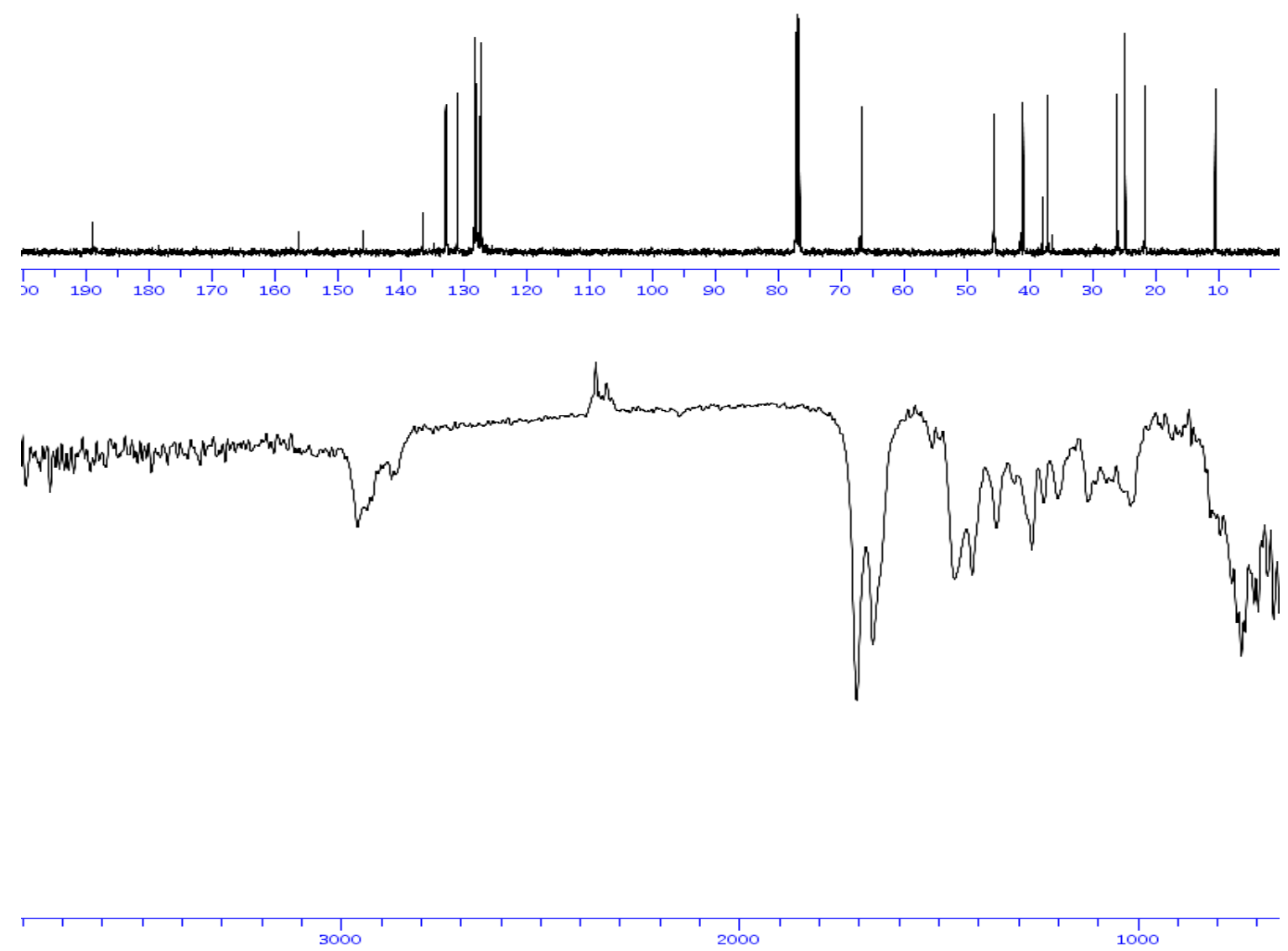

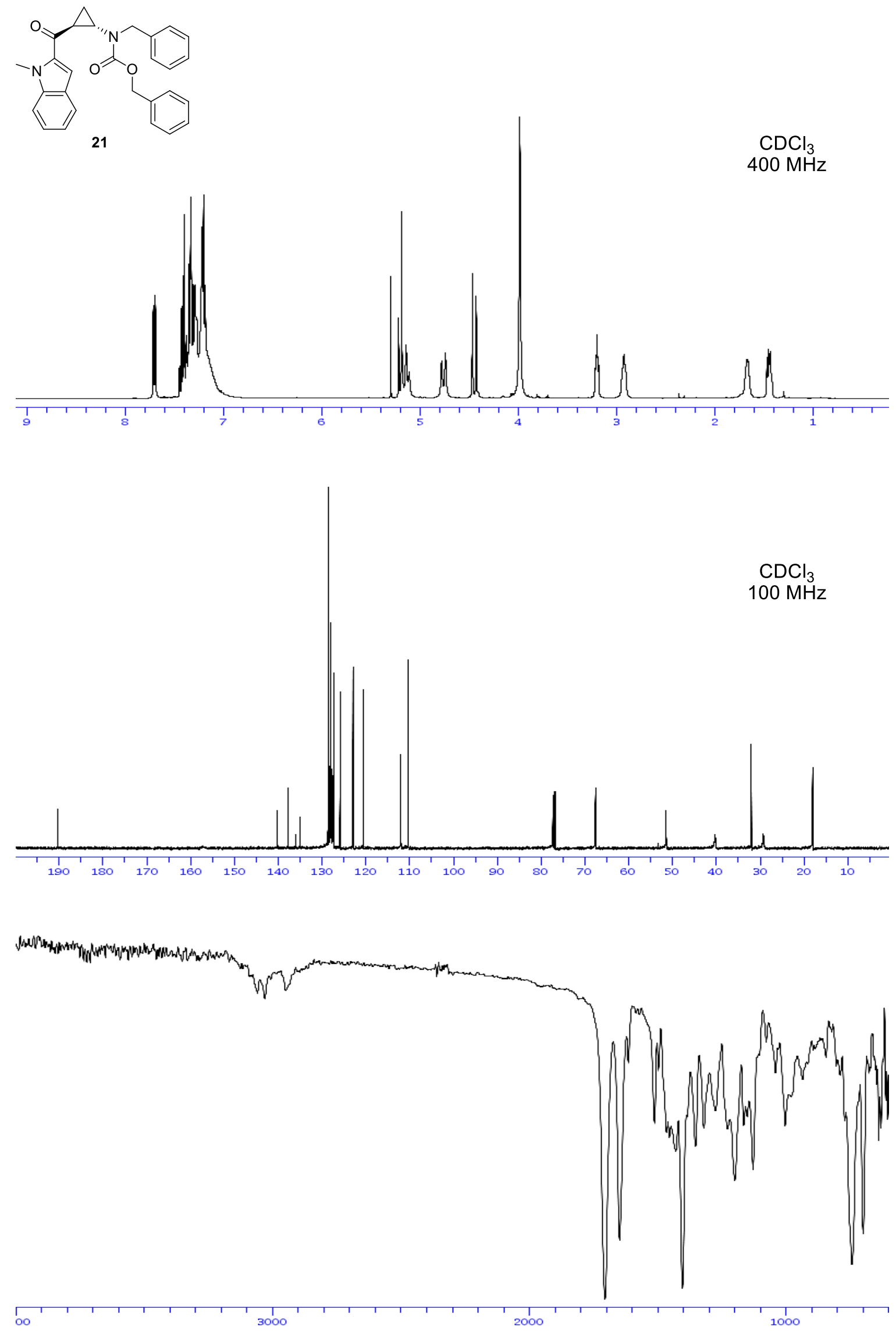

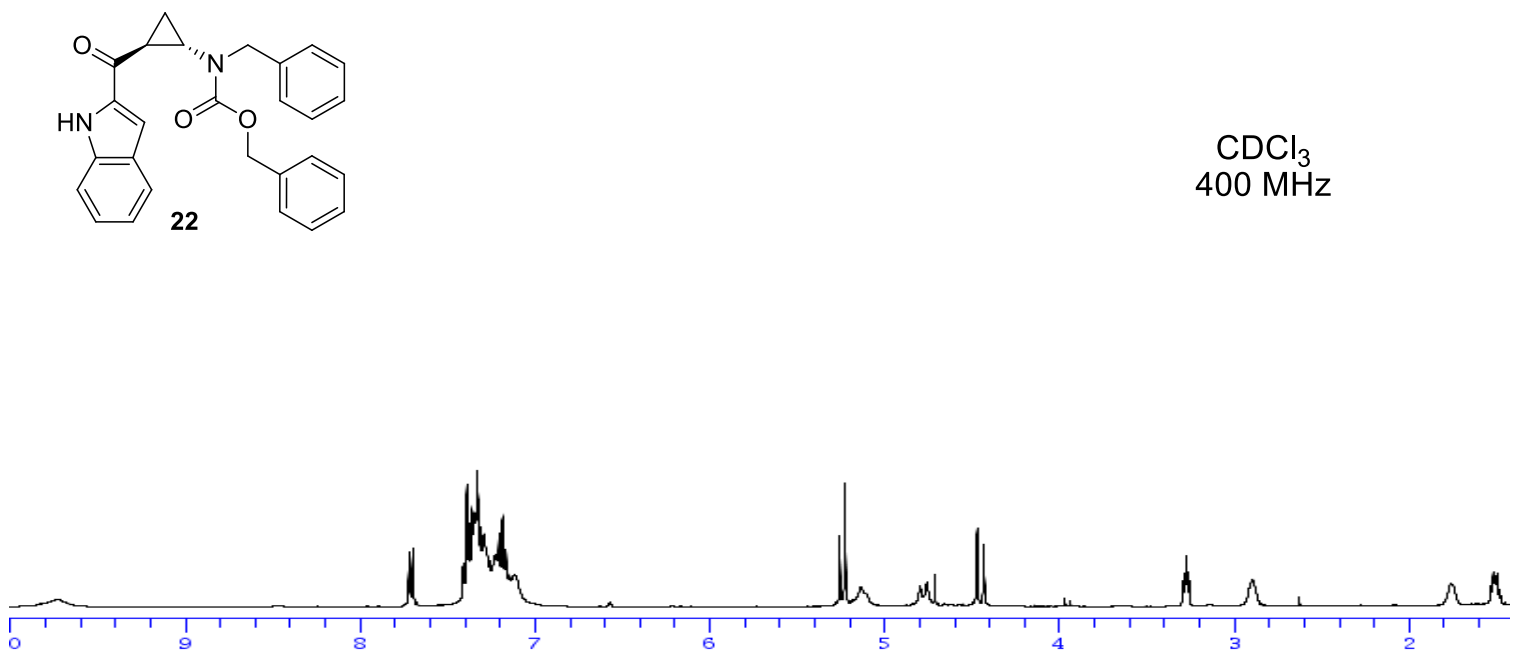

$\mathrm{CDCl}_{3}$

$100 \mathrm{MHz}$
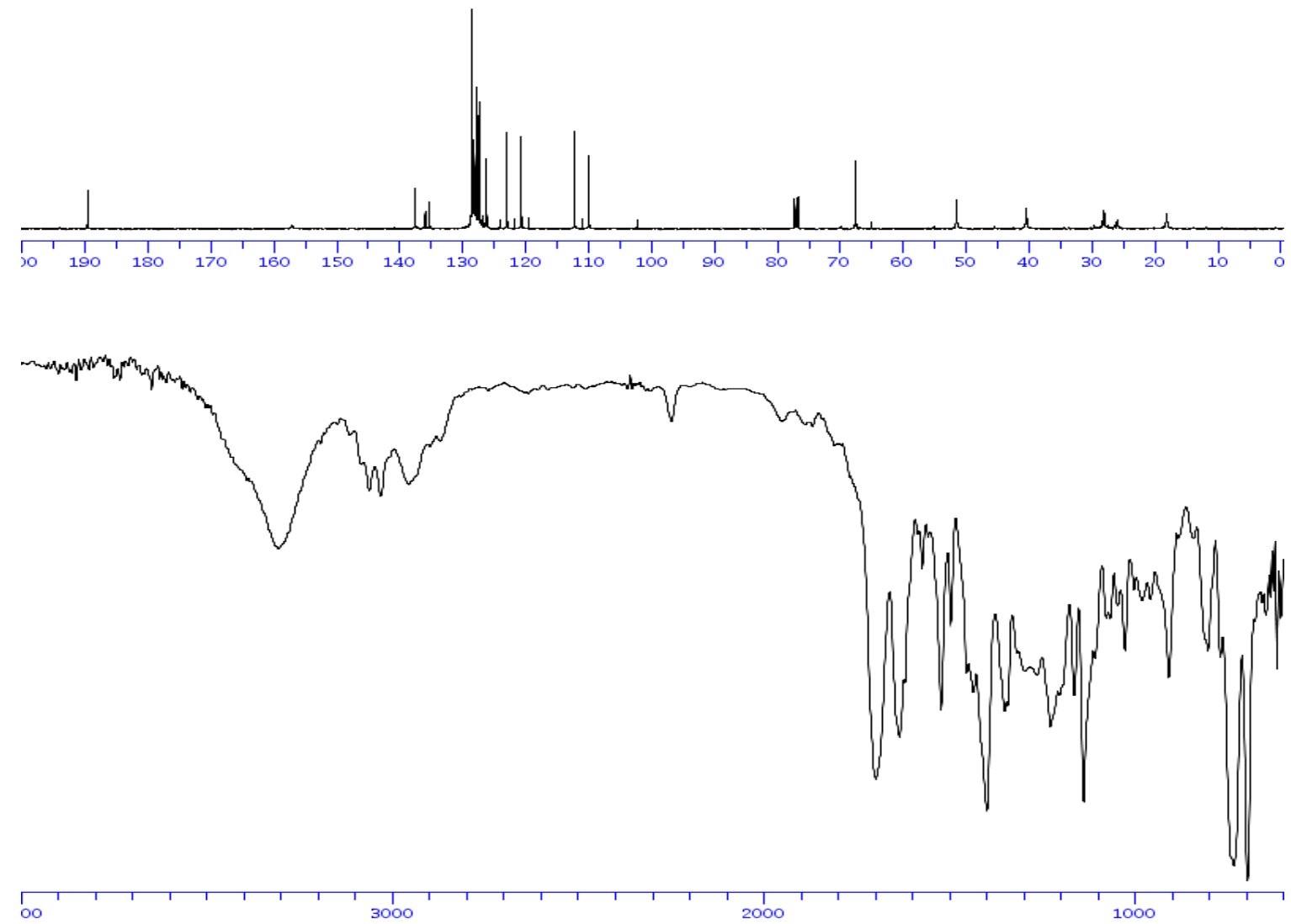


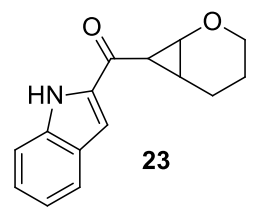

$\mathrm{CDCl}_{3}$

$400 \mathrm{MHz}$

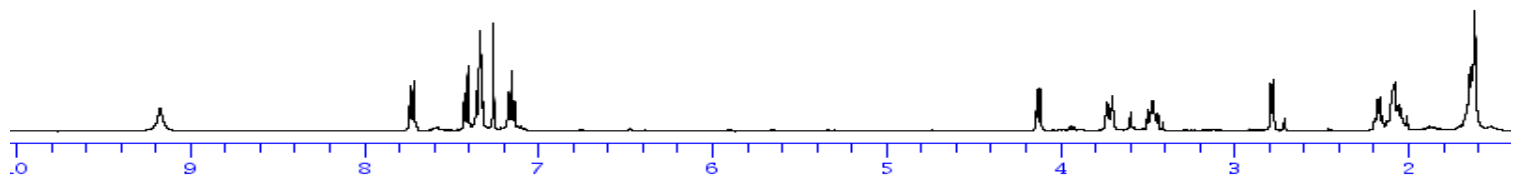

$\mathrm{CDCl}_{3}$

$100 \mathrm{MHz}$
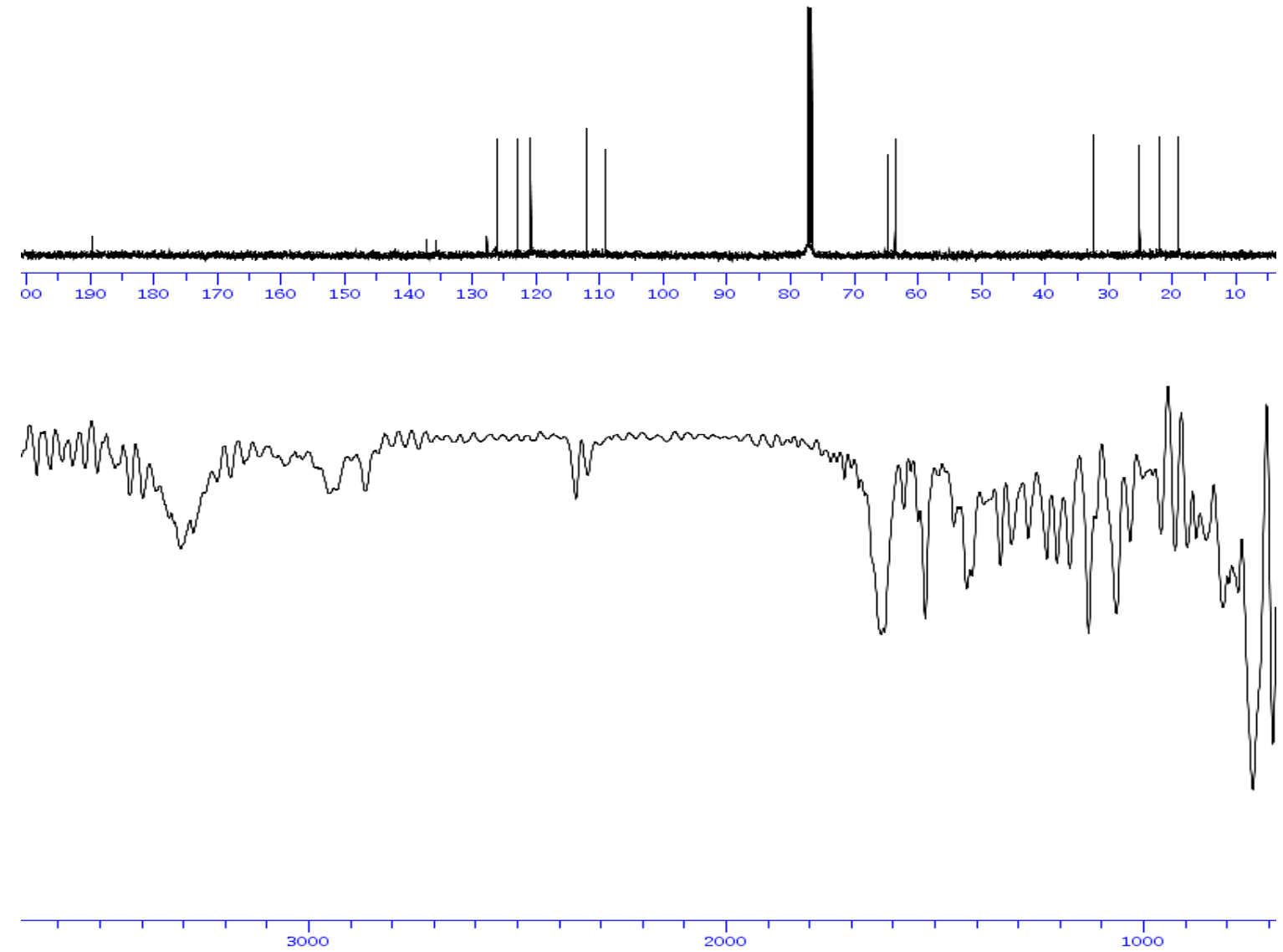


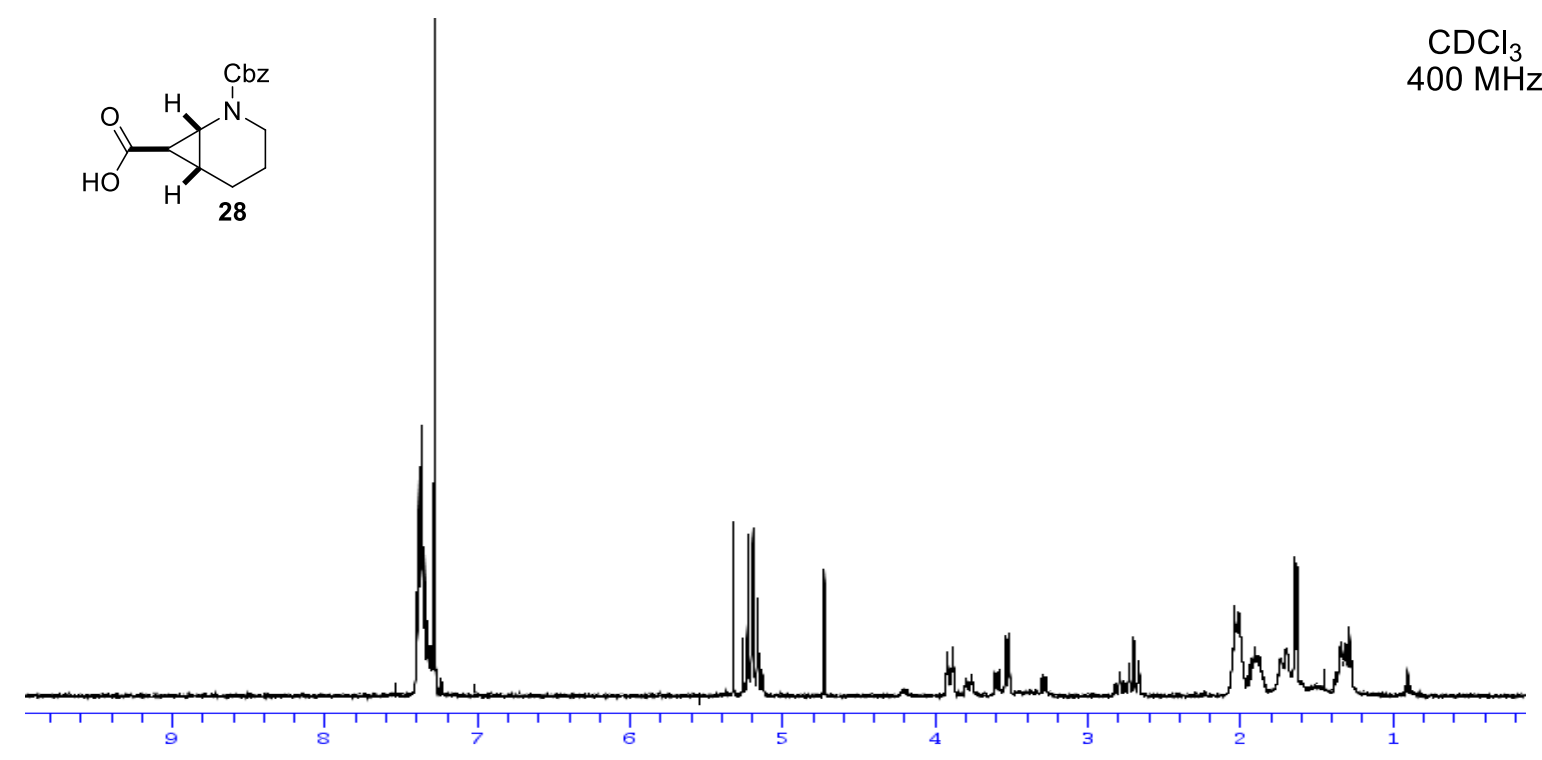

$\mathrm{CDCl}_{3}$

$100 \mathrm{MHz}$

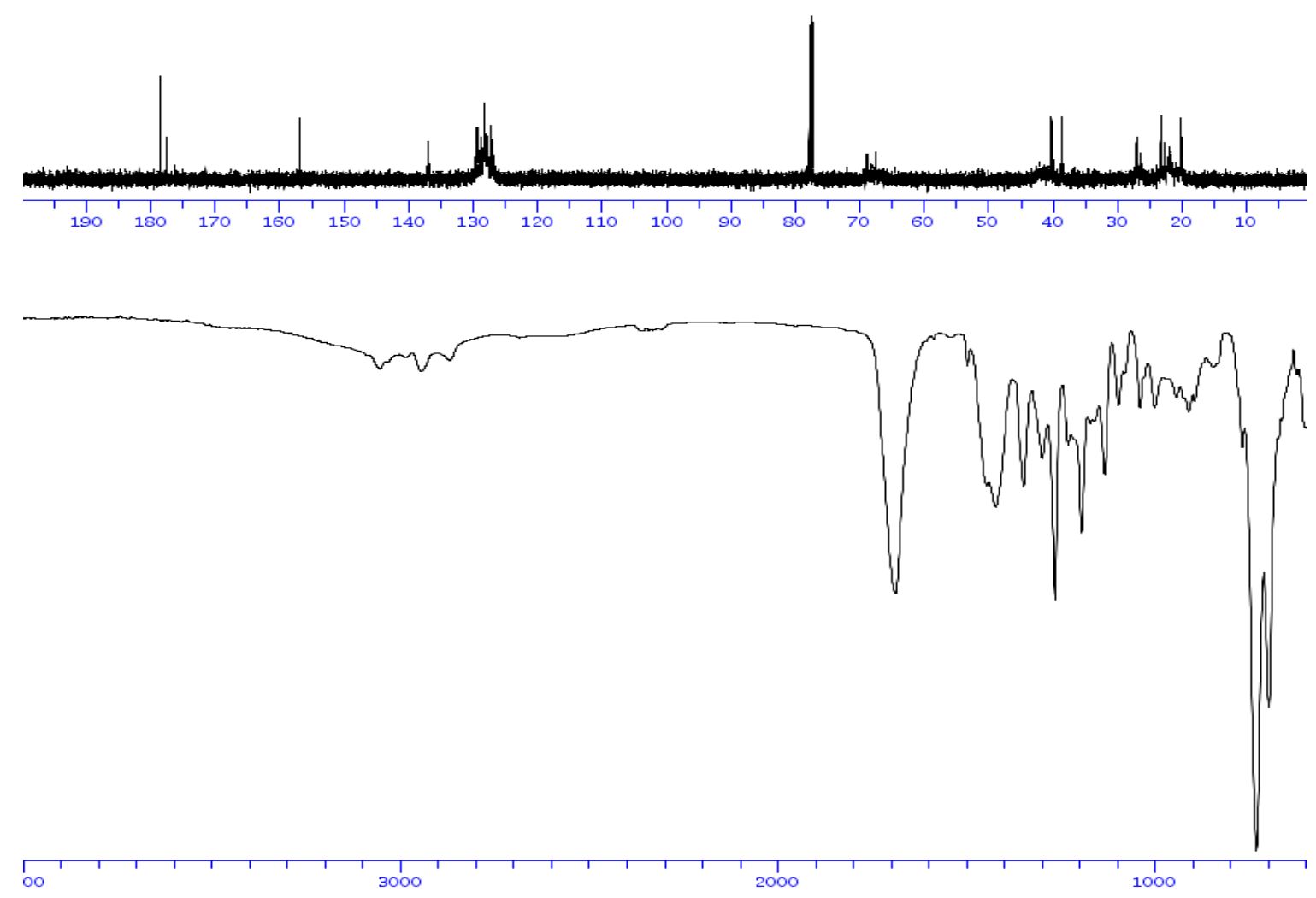




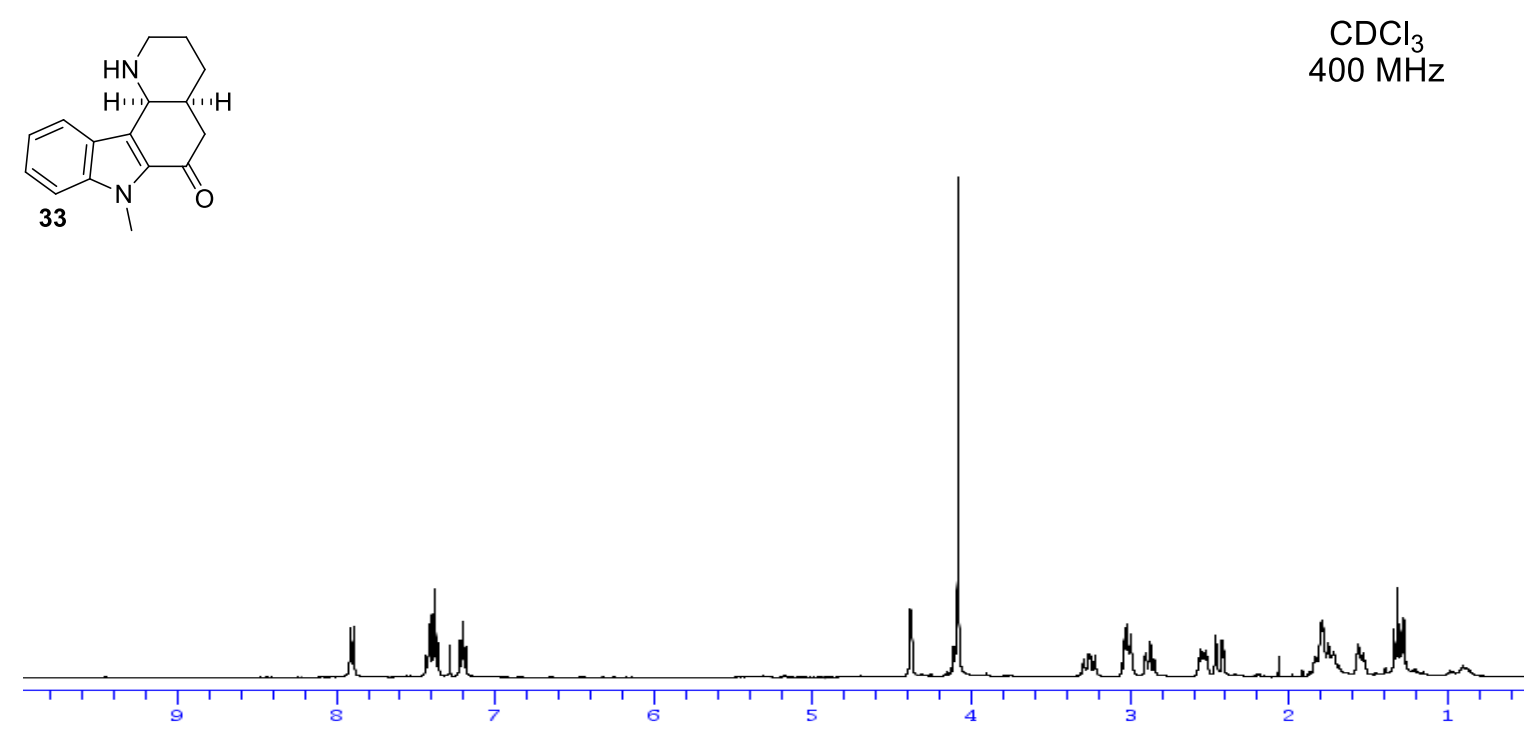

$\mathrm{CDCl}_{3}$

$100 \mathrm{MHz}$
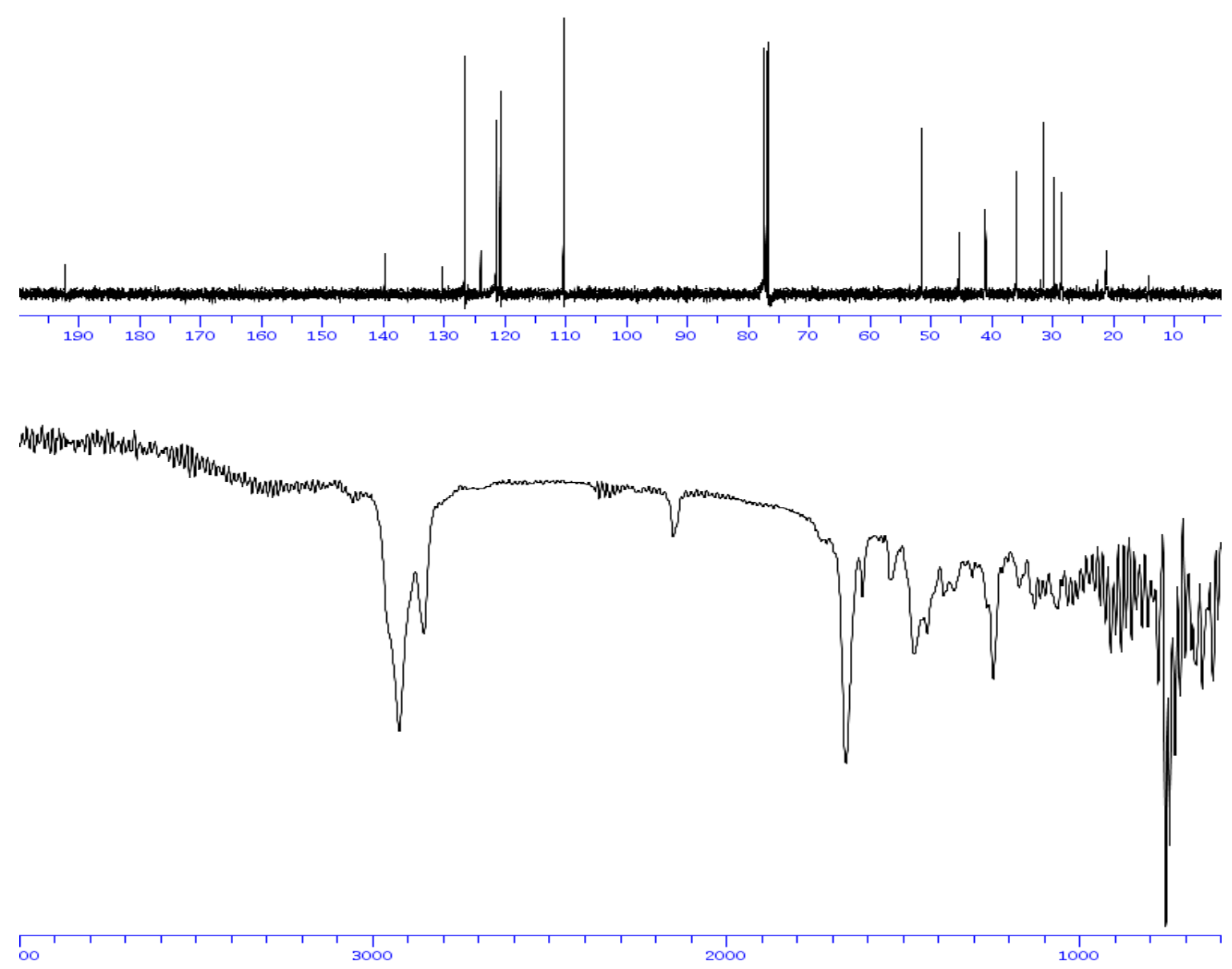

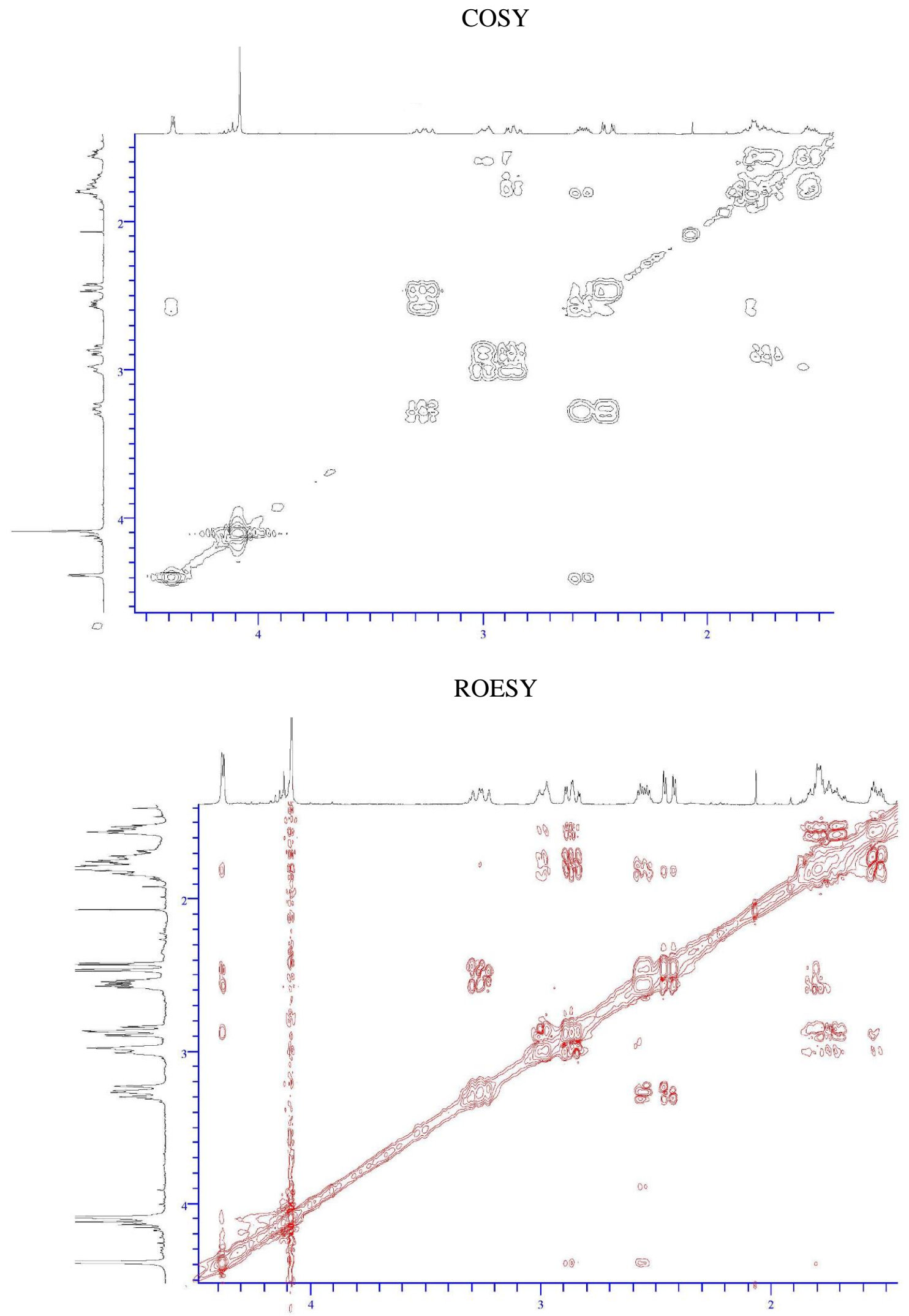
$\mathrm{CDCl}_{3}$

$400 \mathrm{MHz}$
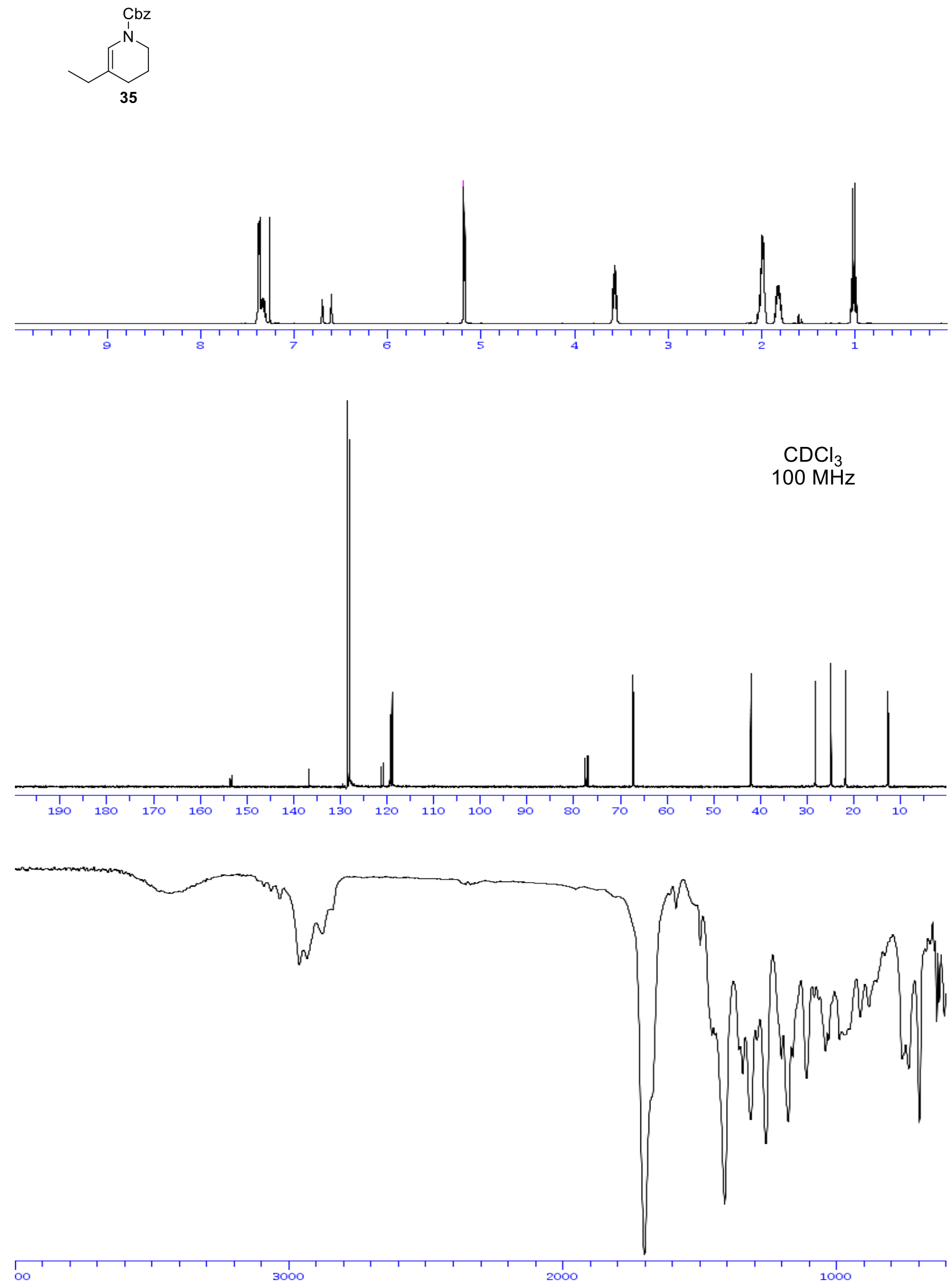

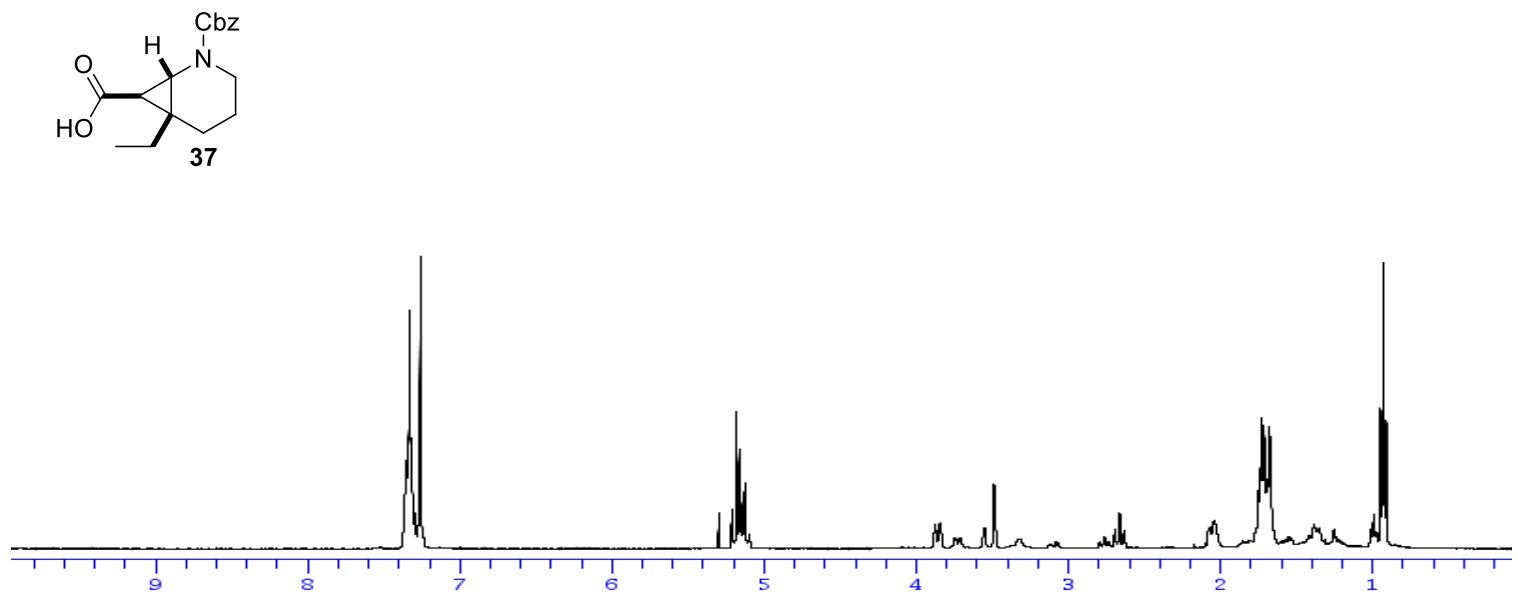

$\mathrm{CDCl}_{3}$

$100 \mathrm{MHz}$
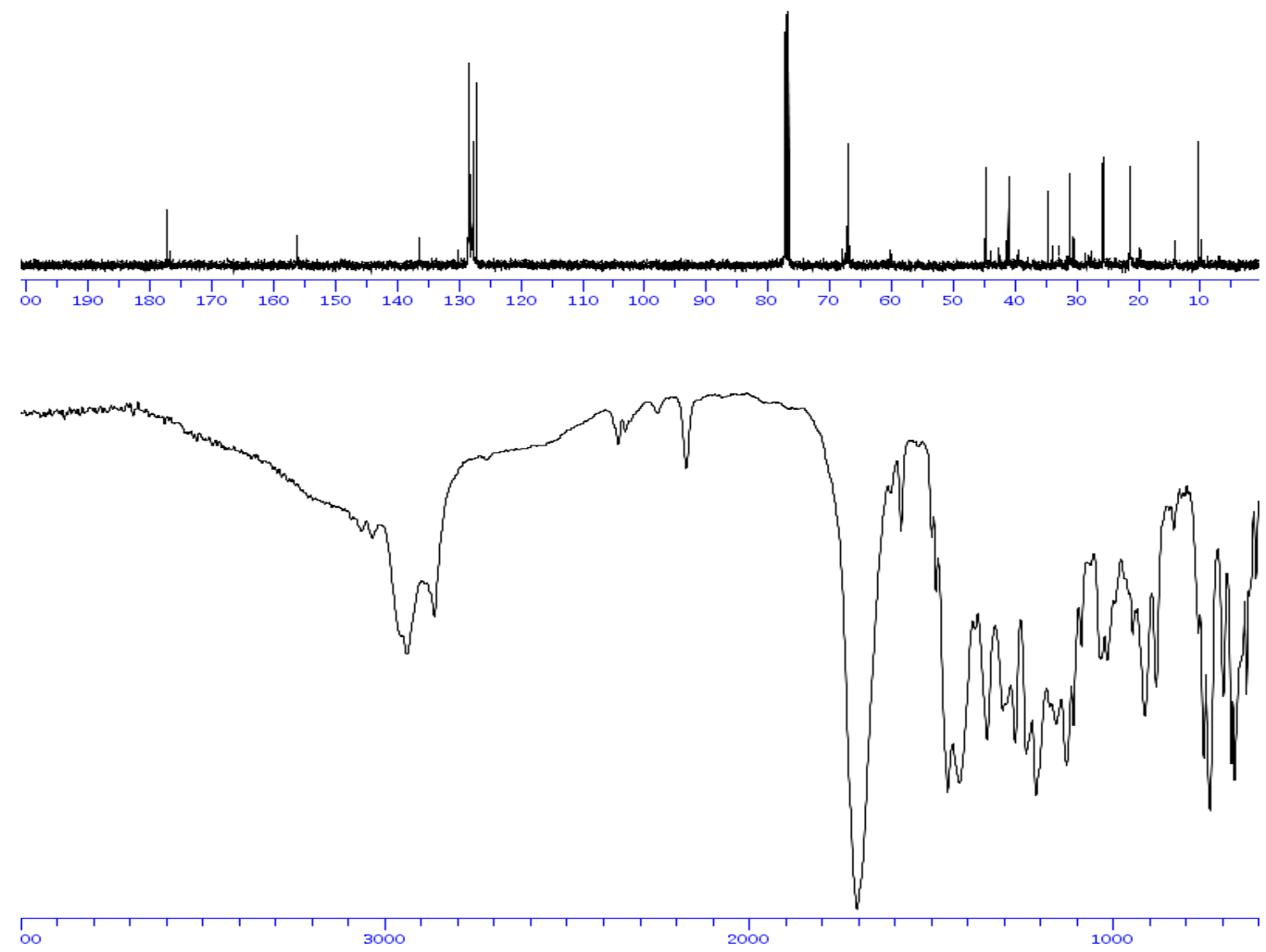


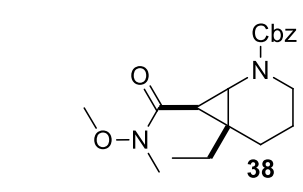

$\mathrm{CDCl}_{3}$

$400 \mathrm{MHz}$
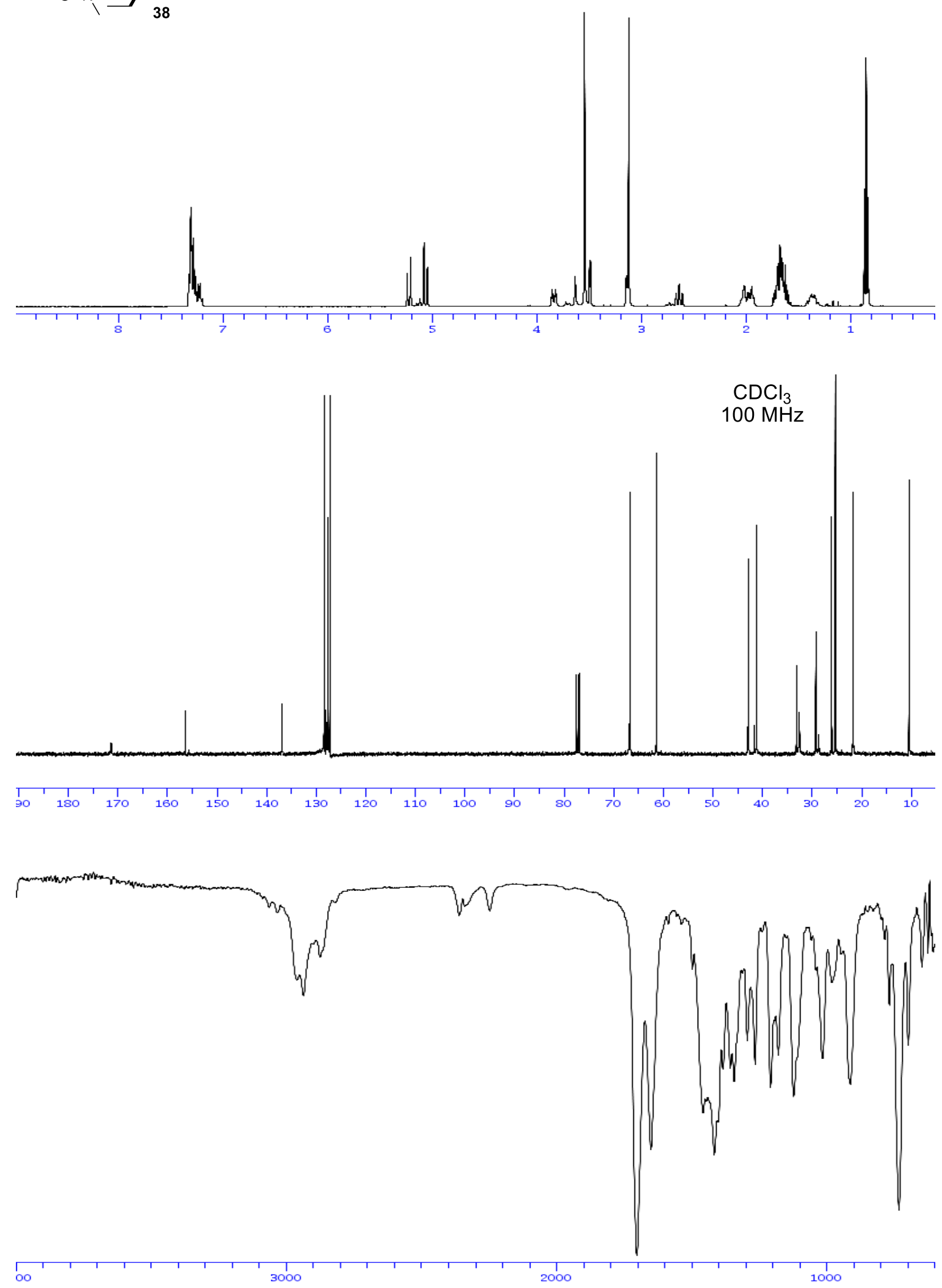


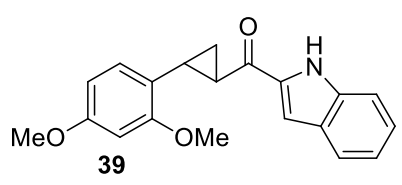

$\mathrm{CDCl}_{3}$

$400 \mathrm{MHz}$
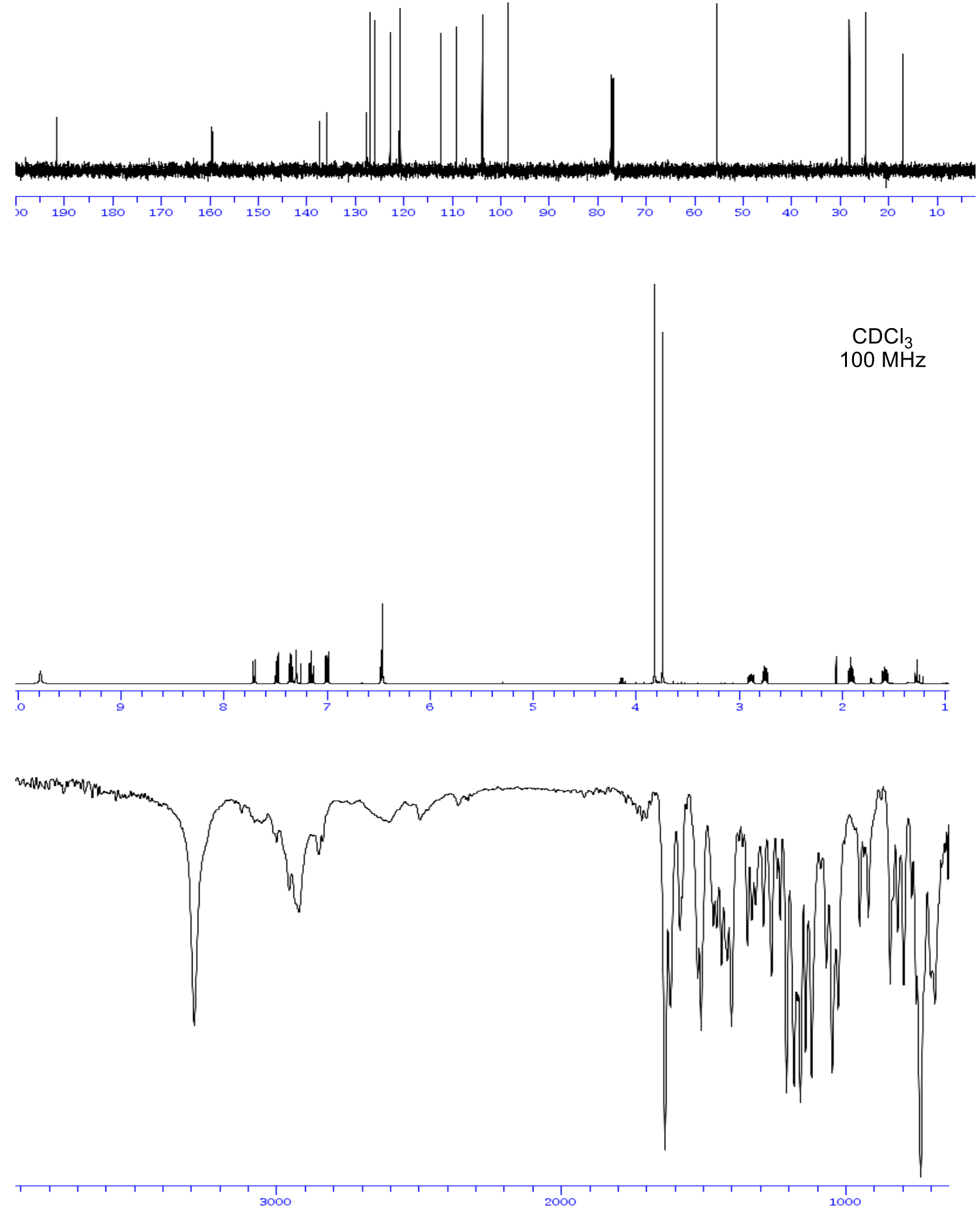


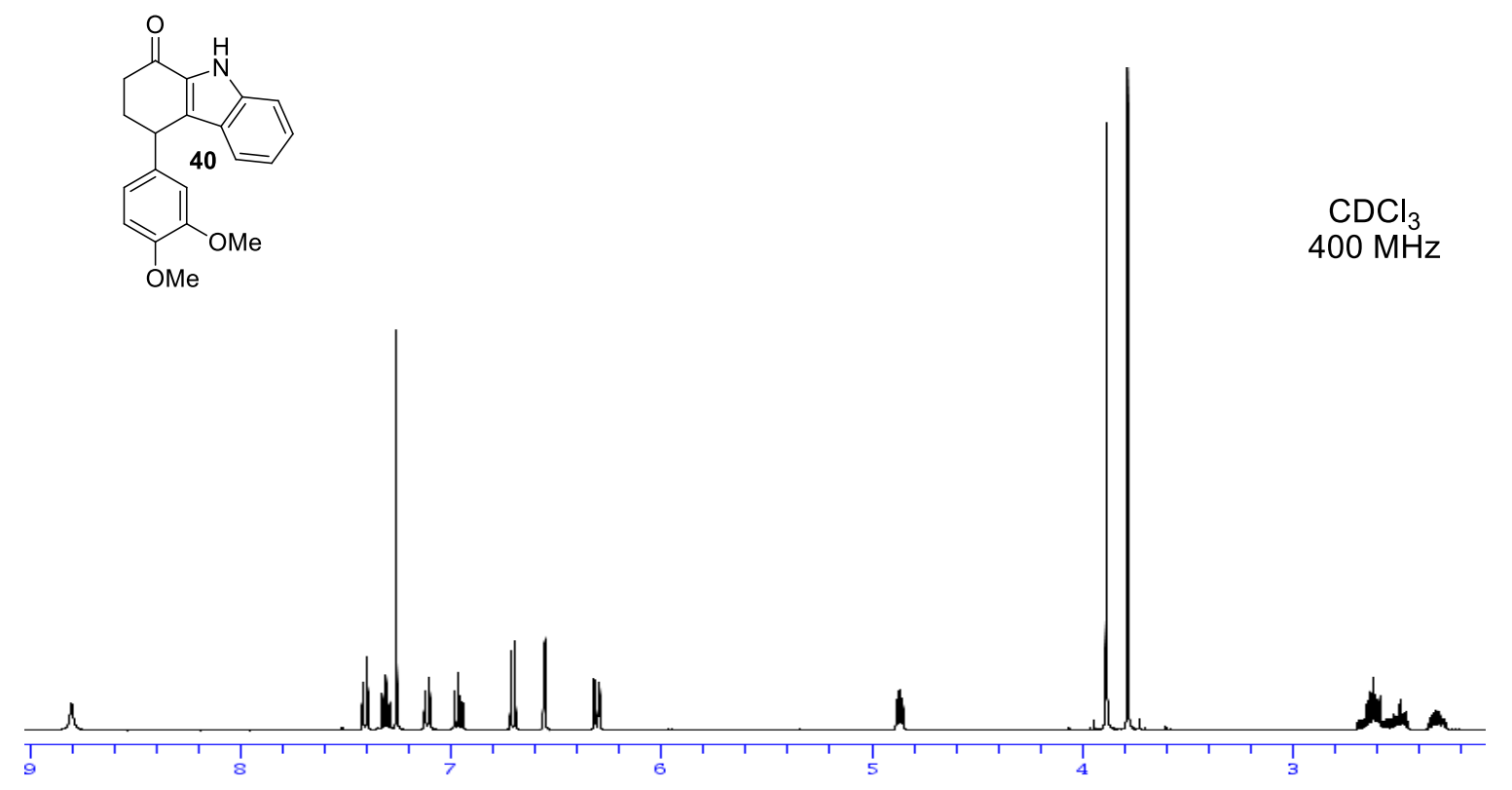

$\mathrm{CDCl}_{3}$

$100 \mathrm{MHz}$
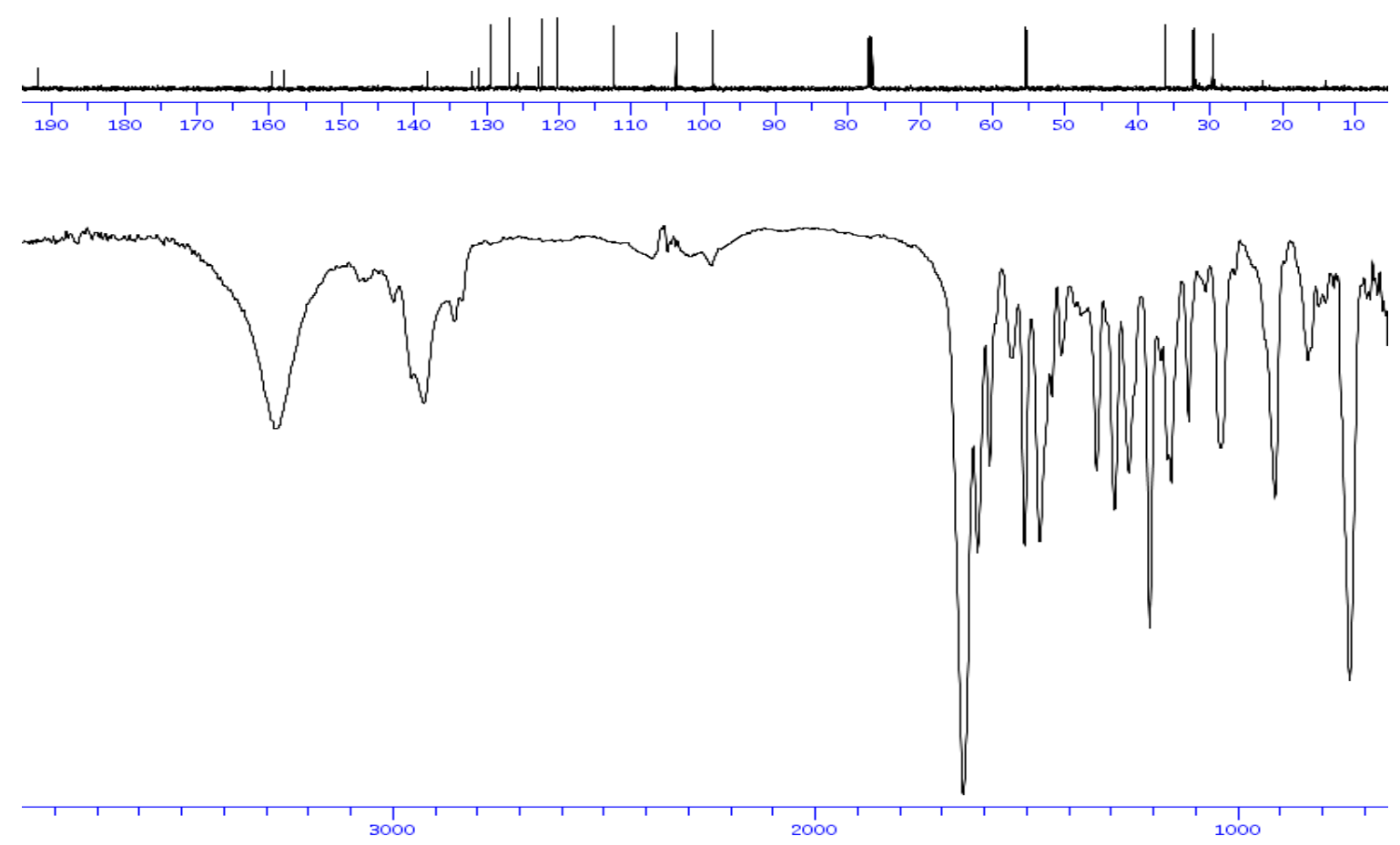


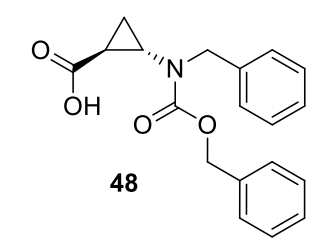

$\mathrm{CDCl}_{3}$

$400 \mathrm{MHz}$

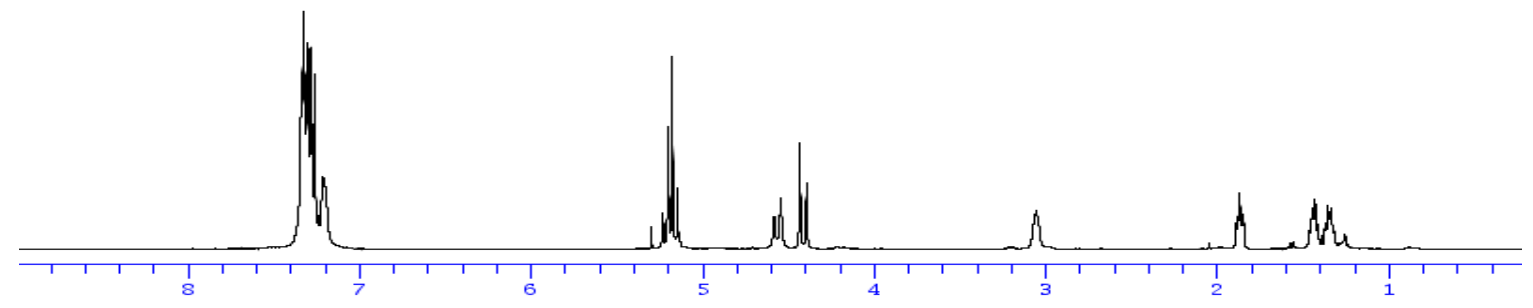

$\mathrm{CDCl}_{3}$

$100 \mathrm{MHz}$
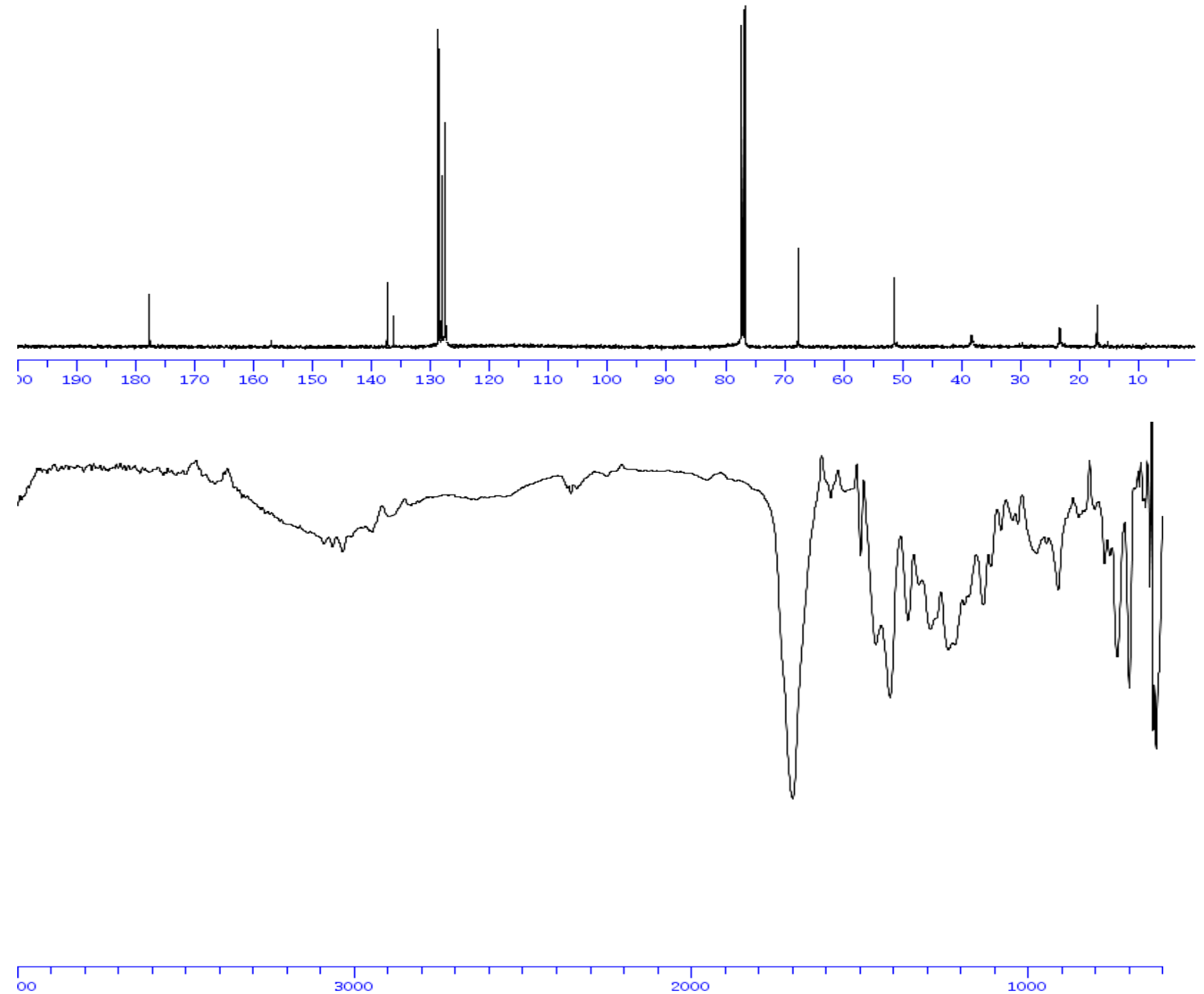

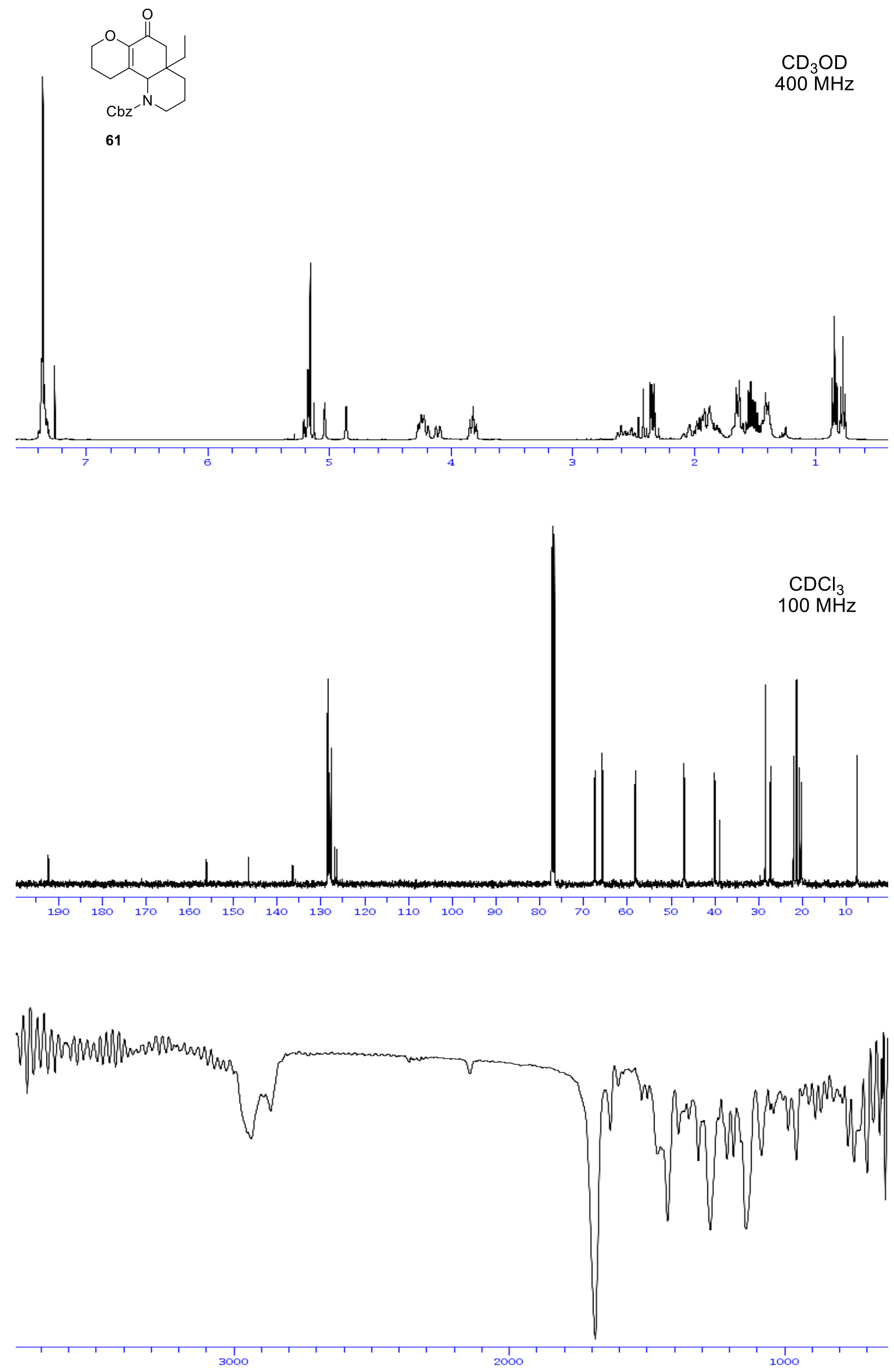

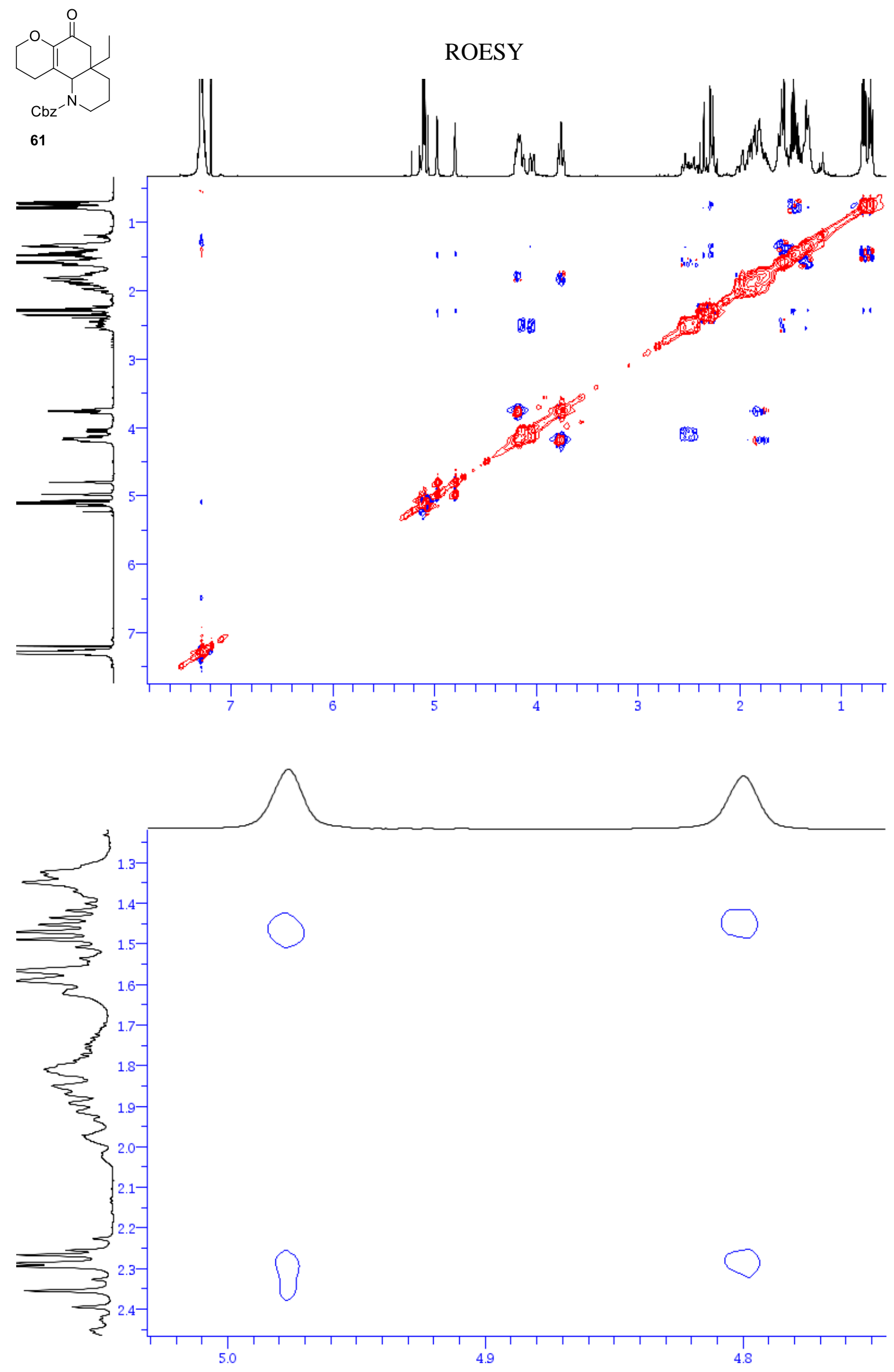


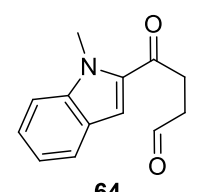

$\mathrm{CD}_{3} \mathrm{OD}$

$400 \mathrm{MHz}$

64

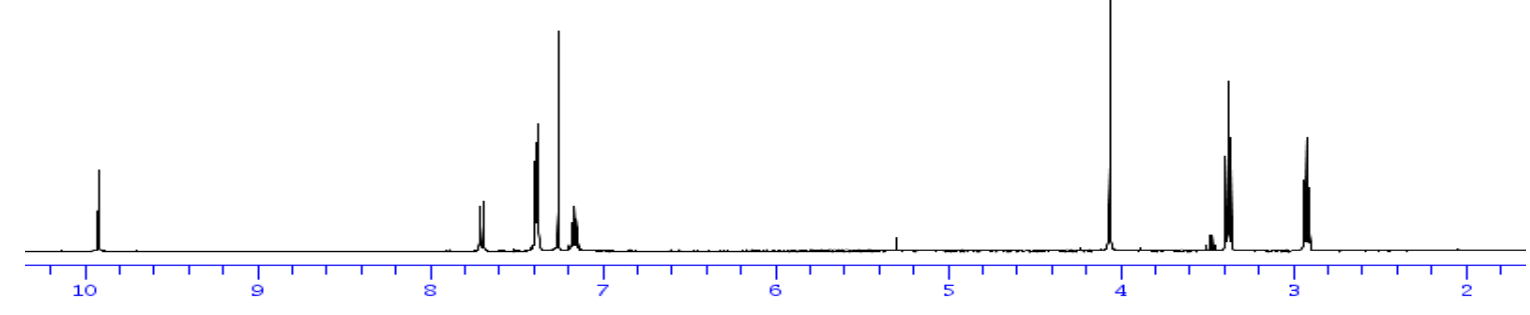

$\mathrm{CDCl}_{3}$

$100 \mathrm{MHz}$

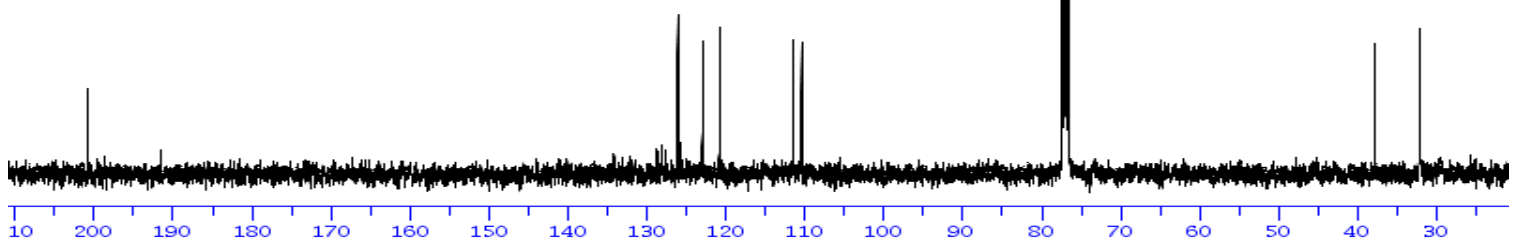

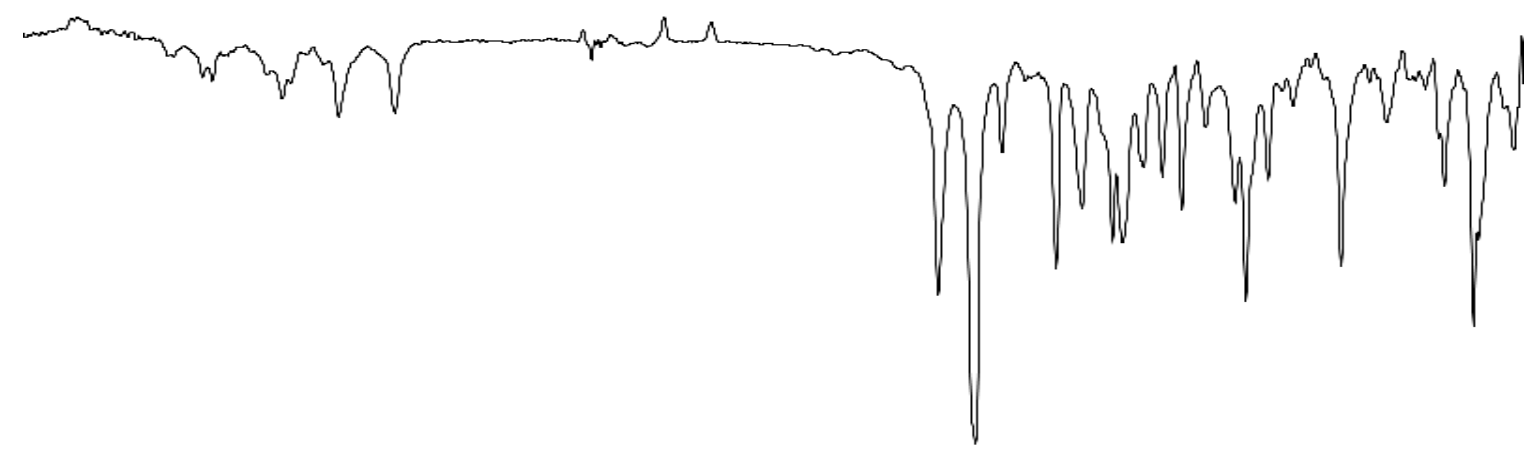



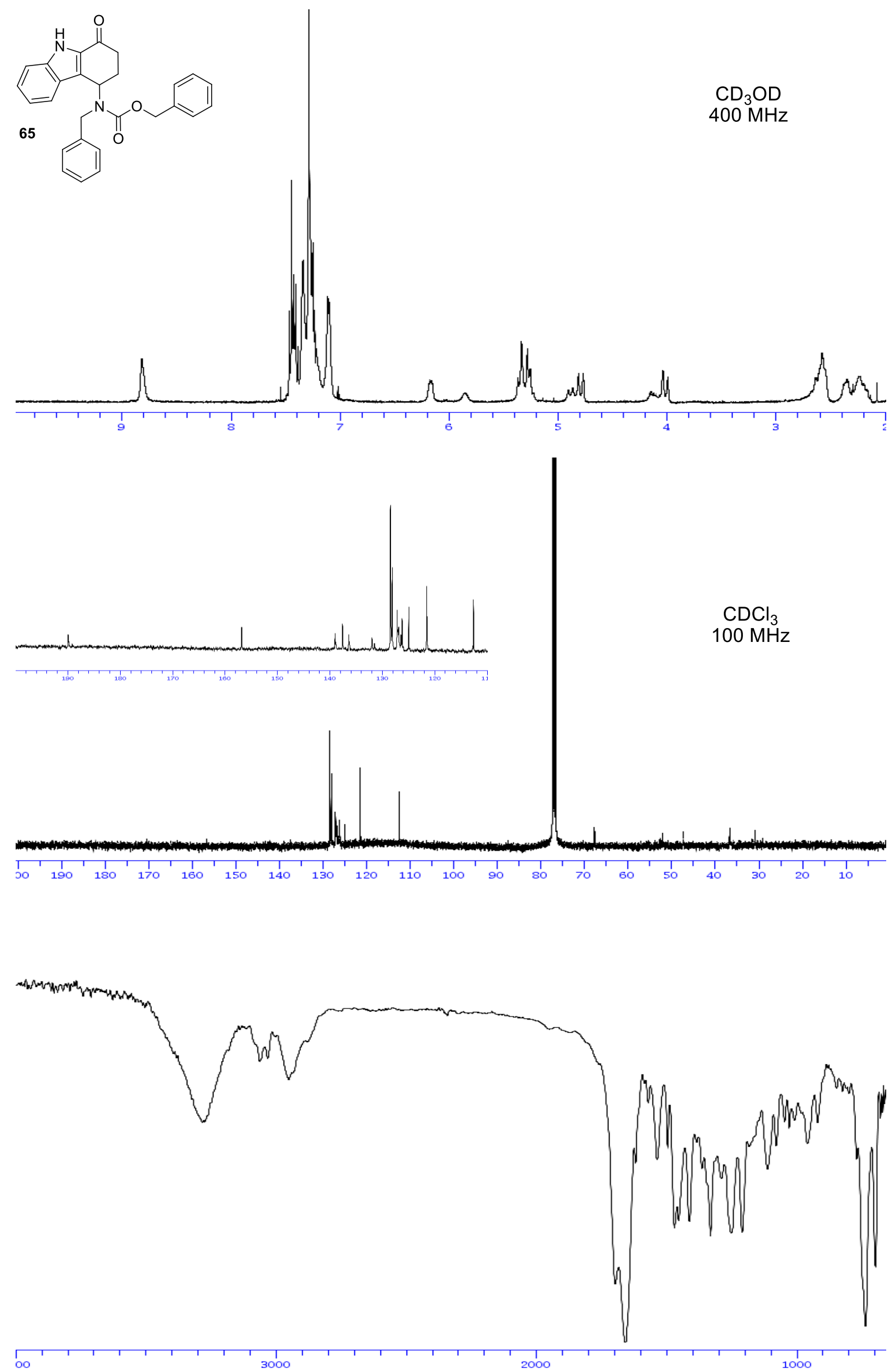

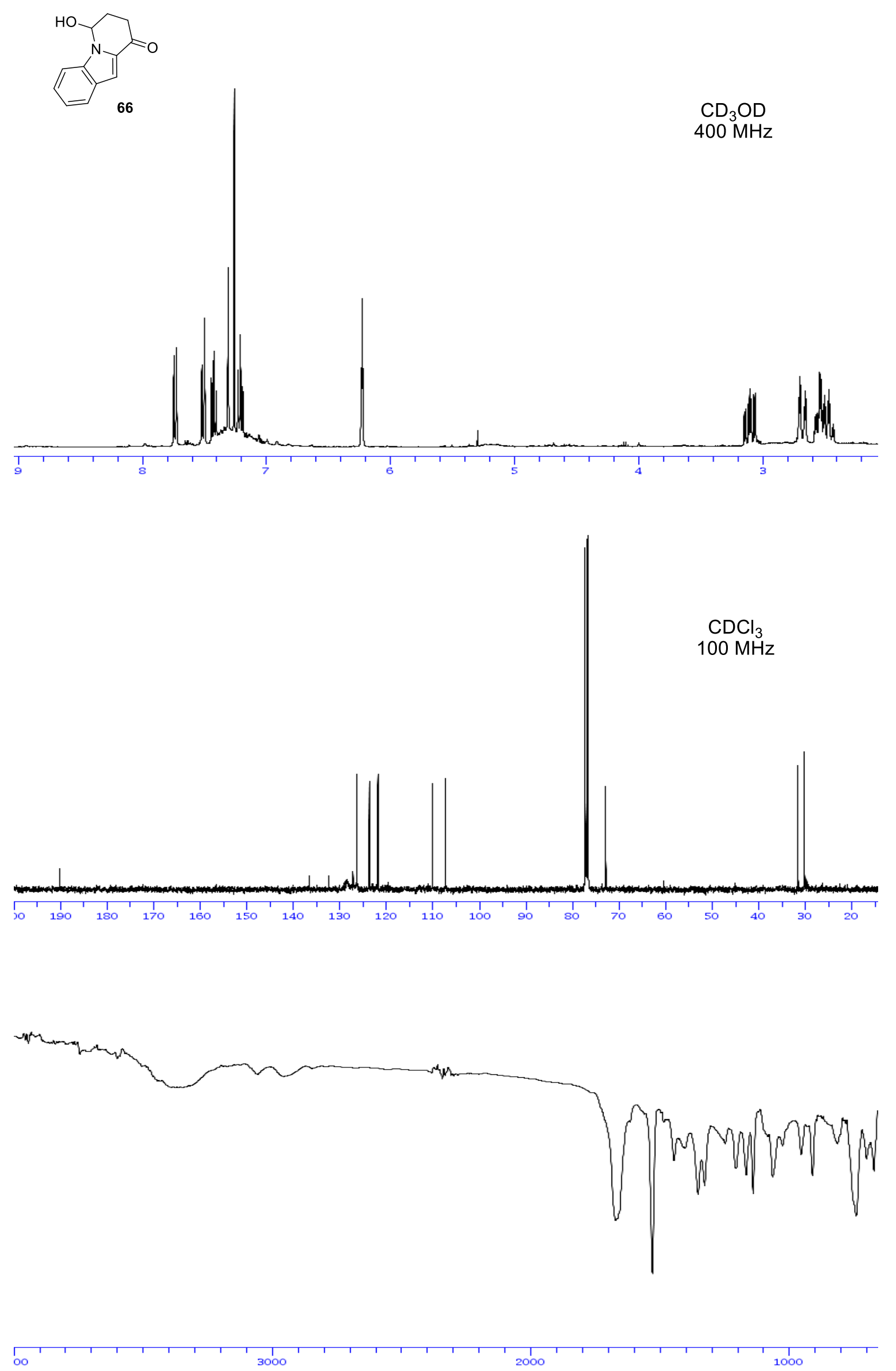


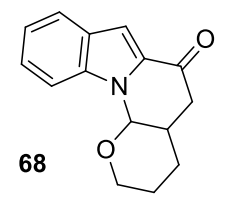

$\mathrm{CD}_{3} \mathrm{OD}$

$400 \mathrm{MHz}$

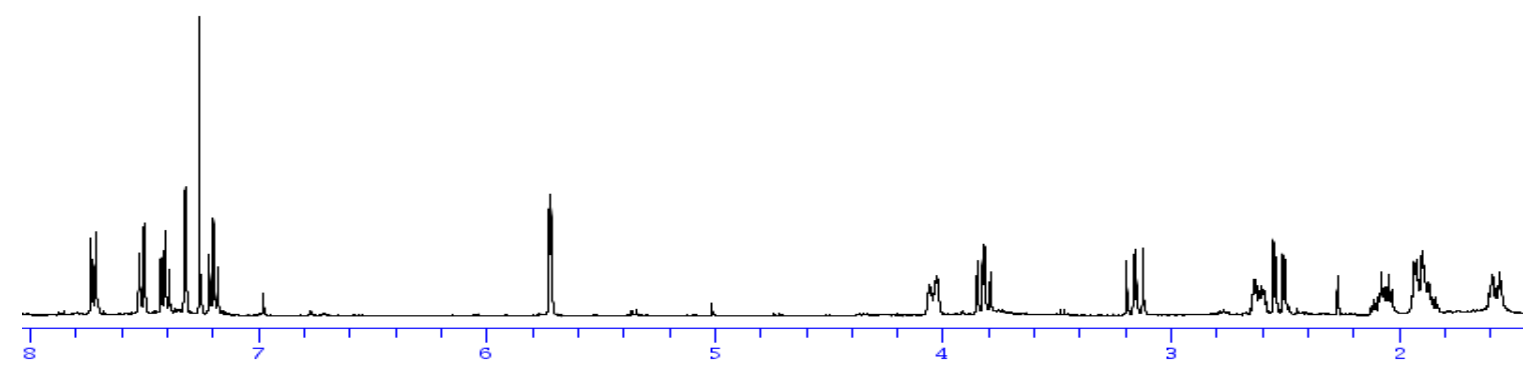

$\mathrm{CDCl}_{3}$

$100 \mathrm{MHz}$
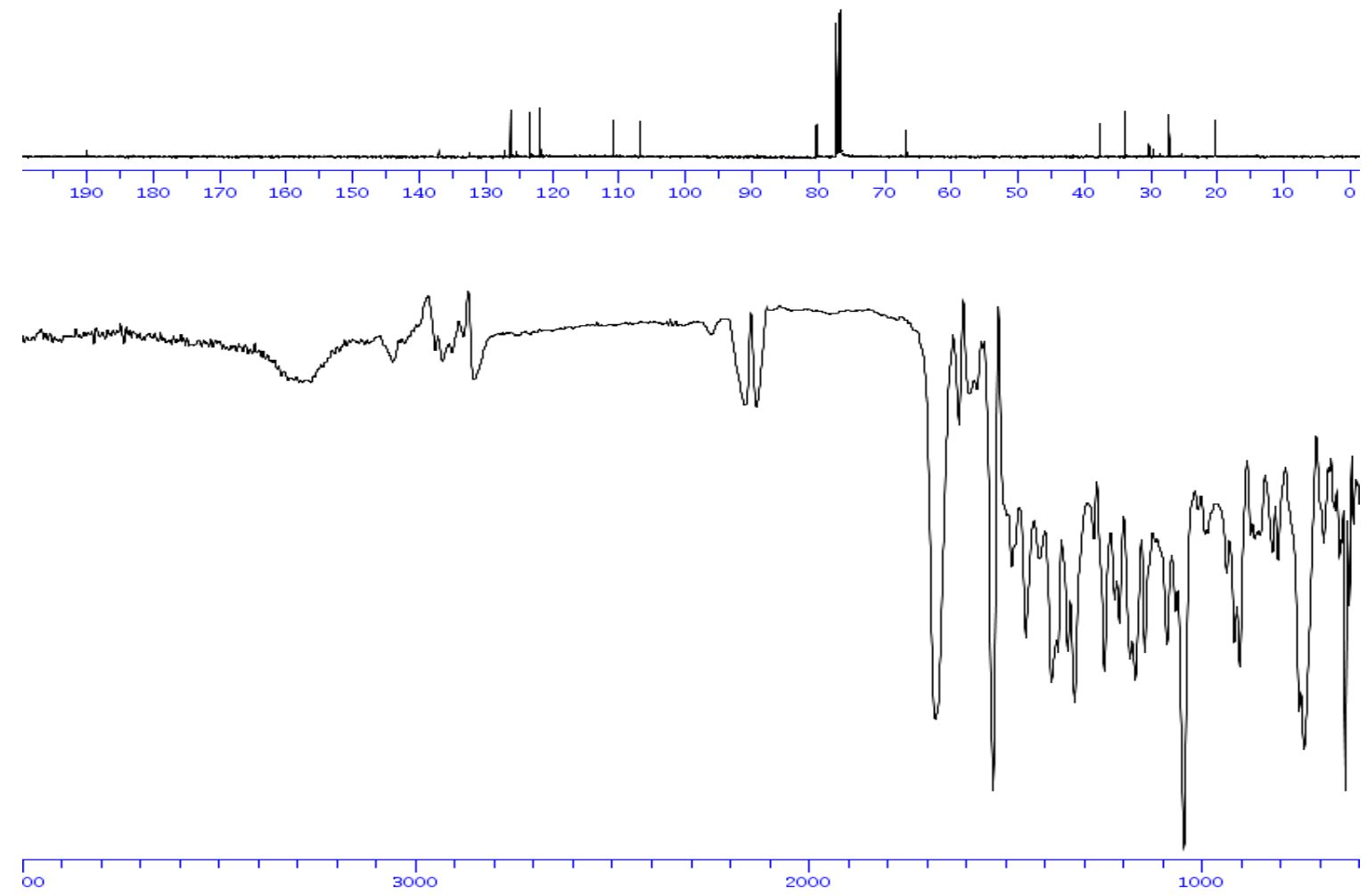
资

ROESY
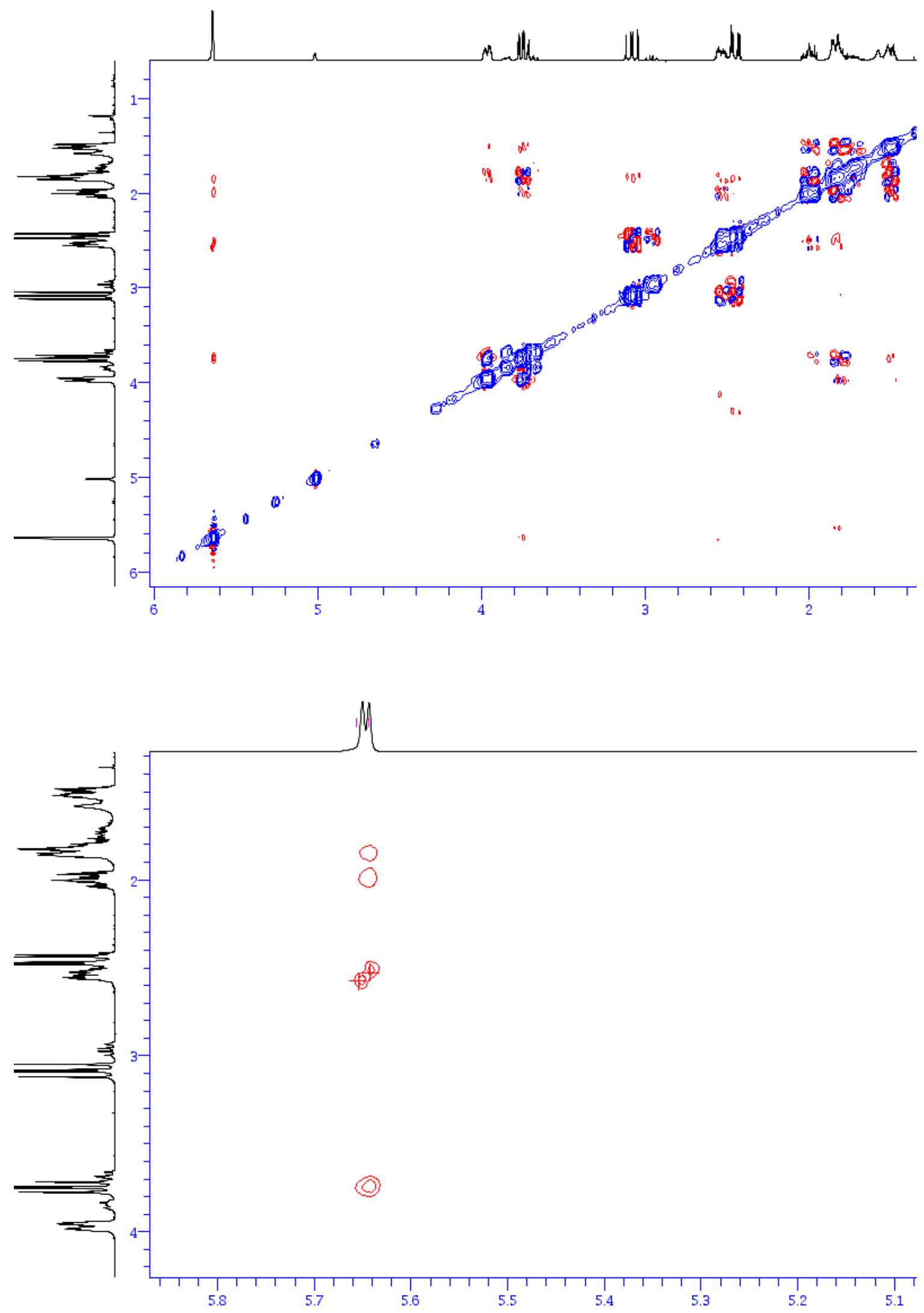


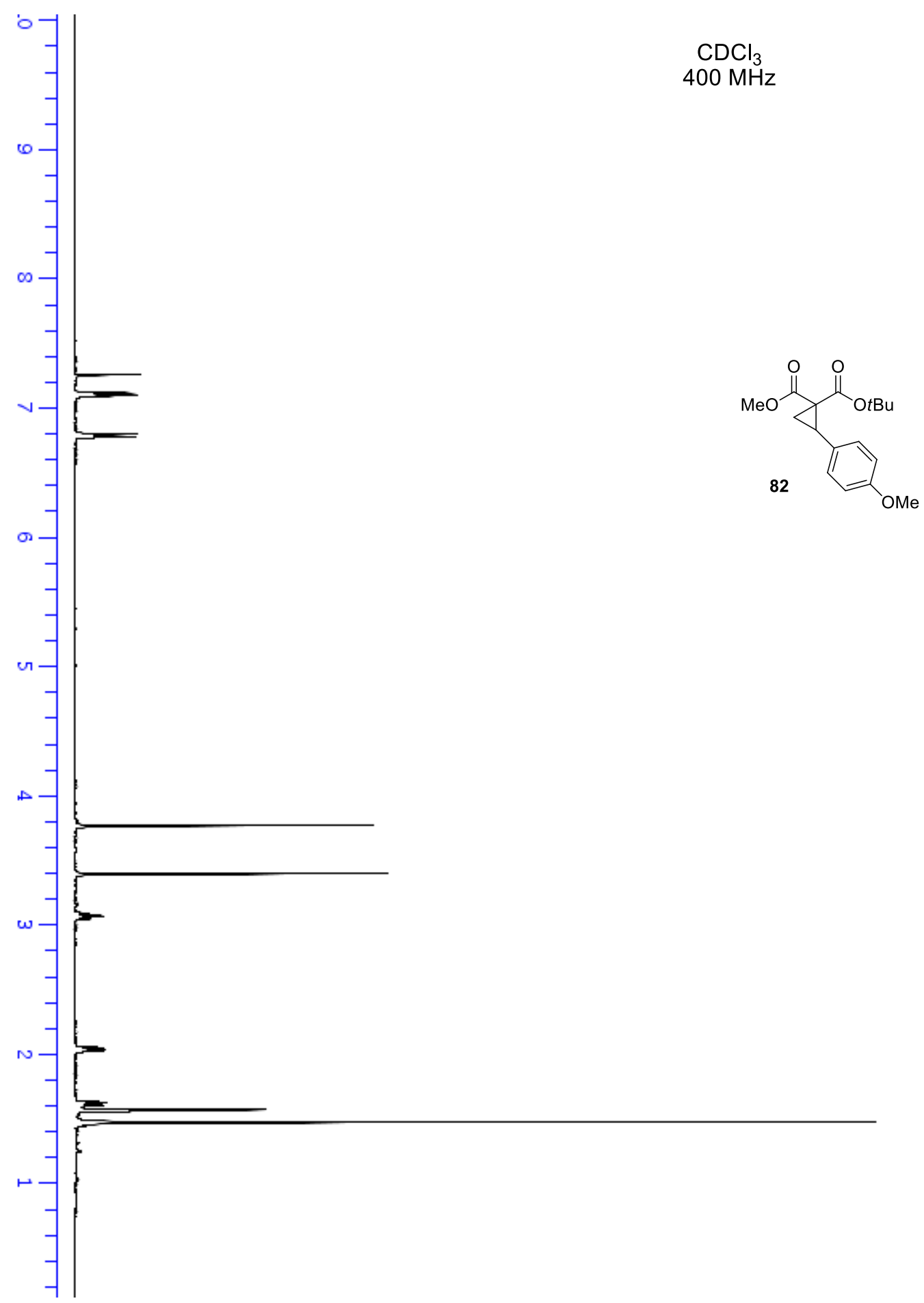




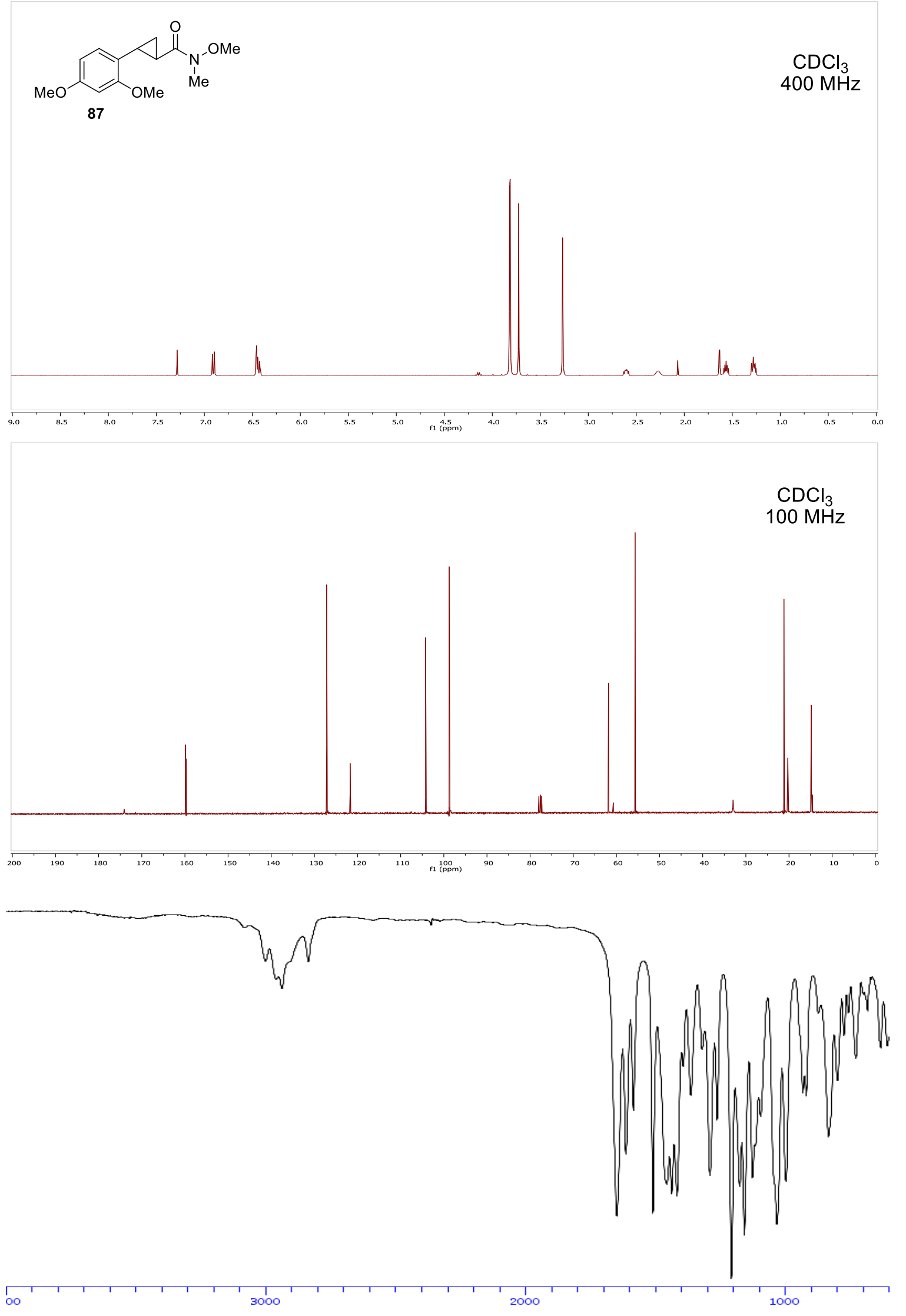




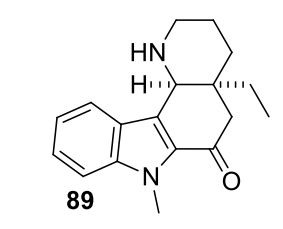

$\mathrm{CDCl}_{3}$

$400 \mathrm{MHz}$
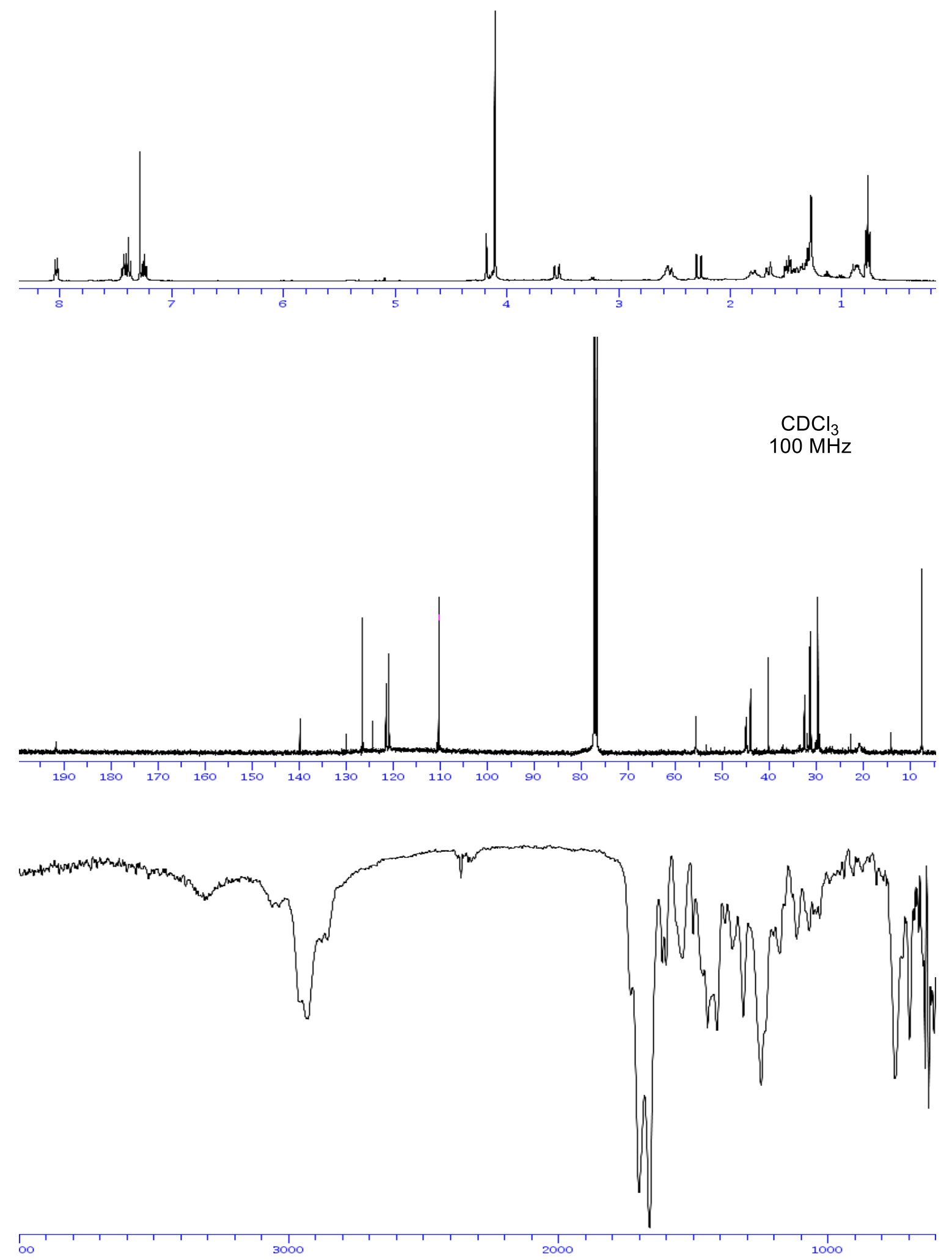


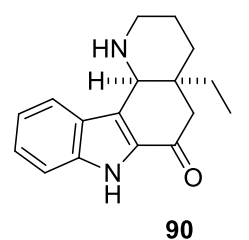

$\mathrm{CD}_{3} \mathrm{OD}$

$400 \mathrm{MHz}$

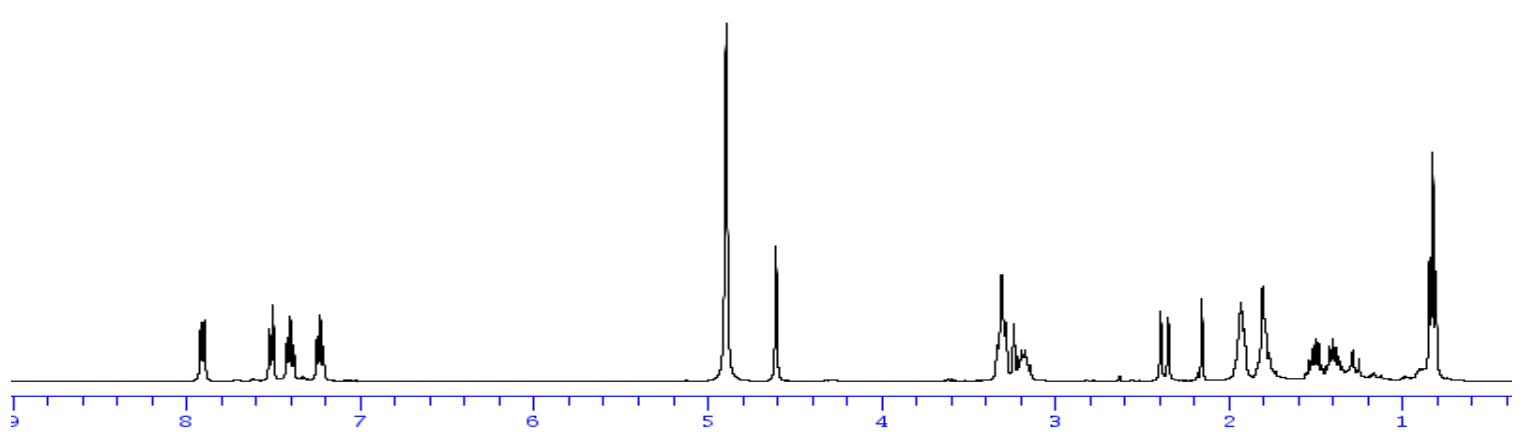

$\mathrm{CDCl}_{3}$

$100 \mathrm{MHz}$
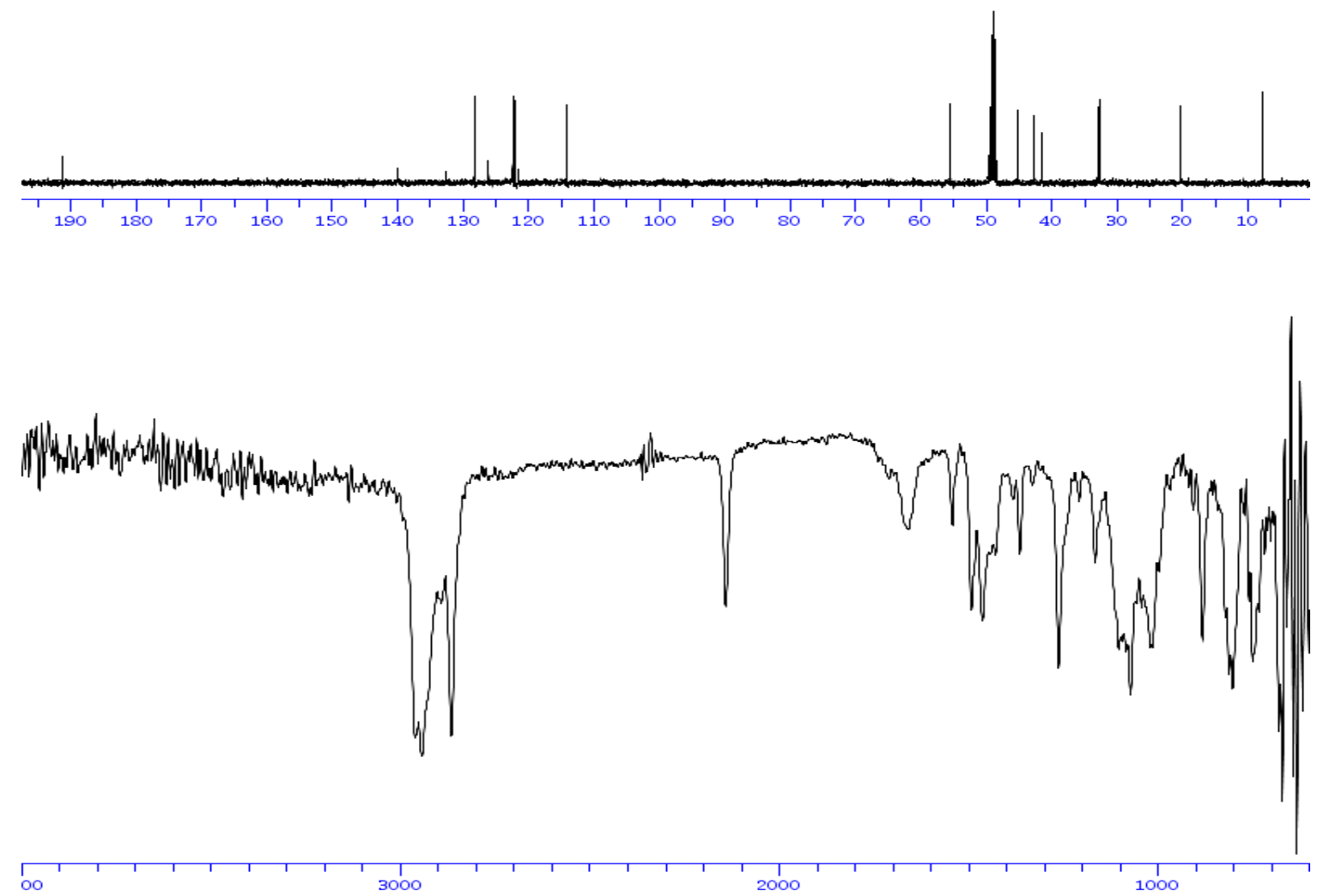
Crystallographic data (CCDC data depository number: 777832).

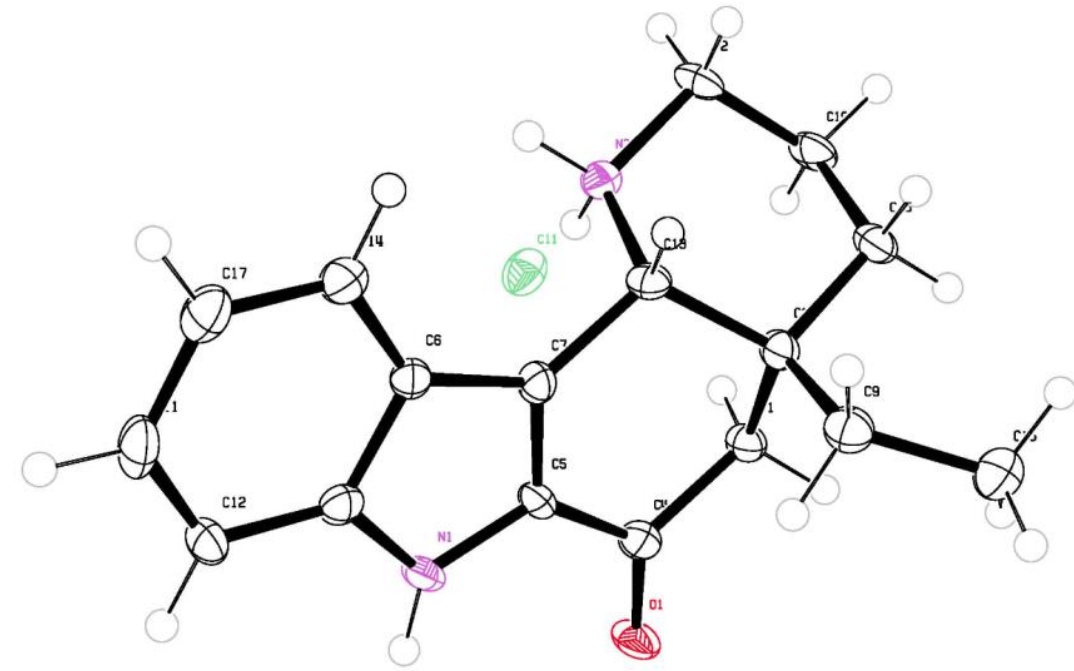

Table 1. Crystal data and structure refinement for fds330.

Identification code

Empirical formula

Formula weight

Temperature

Wavelength

Crystal system

Space group

Unit cell dimensions

Volume

$\mathrm{Z}$

Density (calculated)

Absorption coefficient

$\mathrm{F}(000)$

Crystal size

Theta range for data collection

Index ranges

Reflections collected

Independent reflections

Completeness to theta $=26.37^{\circ}$

Absorption correction

Max. and min. transmission fds 330

$\mathrm{C} 17 \mathrm{H} 21 \mathrm{Cl} \mathrm{N} 2 \mathrm{O}$

304.81

140(2) K

$0.71073 \AA$

Monoclinic

$P 2_{1} / c$

$\mathrm{a}=9.1506(10) \AA$

$\alpha=90^{\circ}$.

$\mathrm{b}=15.3882(14) \AA$

$\beta=103.618(12)^{\circ}$.

$\mathrm{c}=10.9477(12) \AA$

$\gamma=90^{\circ}$.

1498.2(3) $\AA^{3}$

4

\section{$1.351 \mathrm{Mg} / \mathrm{m}^{3}$}

$0.256 \mathrm{~mm}^{-1}$

648

$0.15 \times 0.11 \times 0.09 \mathrm{~mm}^{3}$

2.93 to $26.37^{\circ}$.

$-11<=\mathrm{h}<=11,-19<=\mathrm{k}<=19,-13<=\mathrm{l}<=13$

13092

$3040[\mathrm{R}(\mathrm{int})=0.0708]$

$99.1 \%$

Semi-empirical from equivalents

1.00000 and 0.92803 
Refinement method

Data / restraints / parameters

Goodness-of-fit on $\mathrm{F}^{2}$

Final $\mathrm{R}$ indices [I $>2 \operatorname{sigma}(\mathrm{I})]$

$\mathrm{R}$ indices (all data)

Largest diff. peak and hole
Full-matrix least-squares on $\mathrm{F}^{2}$

3040 / 0 / 274

0.967

$\mathrm{R} 1=0.0546, \mathrm{wR} 2=0.0992$

$\mathrm{R} 1=0.1022, \mathrm{wR} 2=0.1112$

0.380 and -0.237 e. $\AA^{-3}$

Table 2. Atomic coordinates ( x 10 $)$ and equivalent isotropic displacement parameters $\left(\AA^{2} \times 10^{3}\right)$ for fds330. $U(e q)$ is defined as one third of the trace of the orthogonalized $U^{i j}$ tensor.

\begin{tabular}{|c|c|c|c|c|}
\hline & $\mathrm{x}$ & $\mathrm{y}$ & $\mathrm{z}$ & $\mathrm{U}(\mathrm{eq})$ \\
\hline $\mathrm{Cl}(1)$ & $2806(1)$ & $4005(1)$ & $4618(1)$ & $27(1)$ \\
\hline $\mathrm{O}(1)$ & $-709(2)$ & $5221(1)$ & $1532(2)$ & $28(1)$ \\
\hline $\mathrm{N}(1)$ & $3748(3)$ & $5933(2)$ & $4836(2)$ & 19(1) \\
\hline $\mathrm{N}(2)$ & 2019(3) & $5647(2)$ & $754(2)$ & $20(1)$ \\
\hline $\mathrm{C}(1)$ & $2842(3)$ & $6581(2)$ & $3930(2)$ & $17(1)$ \\
\hline$C(2)$ & $3758(3)$ & $6081(2)$ & $6187(3)$ & $23(1)$ \\
\hline $\mathrm{C}(3)$ & $2167(3)$ & $6082(2)$ & $6324(3)$ & $24(1)$ \\
\hline $\mathrm{C}(4)$ & $1247(3)$ & $6764(2)$ & $5501(3)$ & $21(1)$ \\
\hline$C(5)$ & $1213(3)$ & $6668(2)$ & $4094(2)$ & $18(1)$ \\
\hline$C(6)$ & 273(3) & $5867(2)$ & $3555(2)$ & $18(1)$ \\
\hline$C(7)$ & $317(3)$ & $5612(2)$ & $2231(2)$ & $20(1)$ \\
\hline $\mathrm{C}(8)$ & $1700(3)$ & $5838(2)$ & $1889(2)$ & $17(1)$ \\
\hline $\mathrm{C}(9)$ & $2867(3)$ & $6294(2)$ & $2628(2)$ & $17(1)$ \\
\hline$C(10)$ & $3988(3)$ & $6389(2)$ & $1933(2)$ & $17(1)$ \\
\hline $\mathrm{C}(11)$ & $3410(3)$ & $5976(2)$ & $759(2)$ & $18(1)$ \\
\hline$C(12)$ & $4208(3)$ & $5956(2)$ & $-184(3)$ & $23(1)$ \\
\hline$C(13)$ & $5577(3)$ & $6356(2)$ & $69(3)$ & $27(1)$ \\
\hline$C(14)$ & $6179(3)$ & $6768(2)$ & $1222(3)$ & $26(1)$ \\
\hline$C(15)$ & $5402(3)$ & $6784(2)$ & $2151(3)$ & $22(1)$ \\
\hline$C(16)$ & $521(3)$ & $7495(2)$ & $3402(3)$ & $23(1)$ \\
\hline $\mathrm{C}(17)$ & $-898(3)$ & $7839(2)$ & $3712(3)$ & $28(1)$ \\
\hline
\end{tabular}

Table 3. Bond lengths $[\AA]$ and angles $\left[{ }^{\circ}\right]$ for fds330.

$\mathrm{O}(1)-\mathrm{C}(7)$




\begin{tabular}{|c|c|}
\hline $\mathrm{N}(1)-\mathrm{C}(2)$ & $1.495(3)$ \\
\hline $\mathrm{N}(1)-\mathrm{C}(1)$ & $1.510(3)$ \\
\hline $\mathrm{N}(1)-\mathrm{H}(1 \mathrm{~A})$ & $0.91(3)$ \\
\hline $\mathrm{N}(1)-\mathrm{H}(1 \mathrm{~B})$ & $0.95(3)$ \\
\hline $\mathrm{N}(2)-\mathrm{C}(11)$ & $1.369(3)$ \\
\hline $\mathrm{N}(2)-\mathrm{C}(8)$ & $1.373(3)$ \\
\hline $\mathrm{N}(2)-\mathrm{H}(2)$ & $0.88(3)$ \\
\hline $\mathrm{C}(1)-\mathrm{C}(9)$ & 1.497(4) \\
\hline $\mathrm{C}(1)-\mathrm{C}(5)$ & $1.548(3)$ \\
\hline $\mathrm{C}(1)-\mathrm{H}(1)$ & $1.00(2)$ \\
\hline$C(2)-C(3)$ & $1.498(4)$ \\
\hline $\mathrm{C}(2)-\mathrm{H}(2 \mathrm{~A})$ & $0.94(3)$ \\
\hline $\mathrm{C}(2)-\mathrm{H}(2 \mathrm{~B})$ & $0.93(3)$ \\
\hline $\mathrm{C}(3)-\mathrm{C}(4)$ & $1.504(4)$ \\
\hline $\mathrm{C}(3)-\mathrm{H}(3 \mathrm{~A})$ & $0.88(3)$ \\
\hline $\mathrm{C}(3)-\mathrm{H}(3 \mathrm{~B})$ & $1.00(2)$ \\
\hline$C(4)-C(5)$ & $1.541(4)$ \\
\hline $\mathrm{C}(4)-\mathrm{H}(4 \mathrm{~A})$ & $0.98(2)$ \\
\hline $\mathrm{C}(4)-\mathrm{H}(4 \mathrm{~B})$ & $0.96(3)$ \\
\hline$C(5)-C(16)$ & $1.539(4)$ \\
\hline $\mathrm{C}(5)-\mathrm{C}(6)$ & $1.540(4)$ \\
\hline$C(6)-C(7)$ & $1.511(4)$ \\
\hline $\mathrm{C}(6)-\mathrm{H}(6 \mathrm{~A})$ & $0.97(3)$ \\
\hline $\mathrm{C}(6)-\mathrm{H}(6 \mathrm{~B})$ & $0.97(3)$ \\
\hline $\mathrm{C}(7)-\mathrm{C}(8)$ & $1.444(4)$ \\
\hline $\mathrm{C}(8)-\mathrm{C}(9)$ & $1.371(3)$ \\
\hline $\mathrm{C}(9)-\mathrm{C}(10)$ & $1.422(4)$ \\
\hline $\mathrm{C}(10)-\mathrm{C}(15)$ & $1.398(4)$ \\
\hline $\mathrm{C}(10)-\mathrm{C}(11)$ & $1.420(4)$ \\
\hline $\mathrm{C}(11)-\mathrm{C}(12)$ & $1.398(4)$ \\
\hline $\mathrm{C}(12)-\mathrm{C}(13)$ & $1.364(4)$ \\
\hline $\mathrm{C}(12)-\mathrm{H}(12)$ & $0.94(2)$ \\
\hline $\mathrm{C}(13)-\mathrm{C}(14)$ & $1.404(4)$ \\
\hline $\mathrm{C}(13)-\mathrm{H}(13)$ & $0.84(3)$ \\
\hline $\mathrm{C}(14)-\mathrm{C}(15)$ & 1.371(4) \\
\hline $\mathrm{C}(14)-\mathrm{H}(14)$ & $0.93(3)$ \\
\hline $\mathrm{C}(15)-\mathrm{H}(15)$ & $0.99(3)$ \\
\hline $\mathrm{C}(16)-\mathrm{C}(17)$ & $1.513(4)$ \\
\hline
\end{tabular}




\begin{tabular}{|c|c|}
\hline $\mathrm{C}(16)-\mathrm{H}(16 \mathrm{~A})$ & $0.96(3)$ \\
\hline $\mathrm{C}(16)-\mathrm{H}(16 \mathrm{~B})$ & $0.95(3)$ \\
\hline $\mathrm{C}(17)-\mathrm{H}(17 \mathrm{~A})$ & $0.92(3)$ \\
\hline $\mathrm{C}(17)-\mathrm{H}(17 \mathrm{~B})$ & $0.95(3)$ \\
\hline $\mathrm{C}(17)-\mathrm{H}(17 \mathrm{C})$ & $0.96(3)$ \\
\hline $\mathrm{C}(2)-\mathrm{N}(1)-\mathrm{C}(1)$ & $115.0(2)$ \\
\hline $\mathrm{C}(2)-\mathrm{N}(1)-\mathrm{H}(1 \mathrm{~A})$ & $104.3(15)$ \\
\hline $\mathrm{C}(1)-\mathrm{N}(1)-\mathrm{H}(1 \mathrm{~A})$ & 112.1(16) \\
\hline $\mathrm{C}(2)-\mathrm{N}(1)-\mathrm{H}(1 \mathrm{~B})$ & $106.6(19)$ \\
\hline $\mathrm{C}(1)-\mathrm{N}(1)-\mathrm{H}(1 \mathrm{~B})$ & 108.9(19) \\
\hline $\mathrm{H}(1 \mathrm{~A})-\mathrm{N}(1)-\mathrm{H}(1 \mathrm{~B})$ & $110(2)$ \\
\hline $\mathrm{C}(11)-\mathrm{N}(2)-\mathrm{C}(8)$ & $108.3(2)$ \\
\hline $\mathrm{C}(11)-\mathrm{N}(2)-\mathrm{H}(2)$ & $123(2)$ \\
\hline $\mathrm{C}(8)-\mathrm{N}(2)-\mathrm{H}(2)$ & $129(2)$ \\
\hline $\mathrm{C}(9)-\mathrm{C}(1)-\mathrm{N}(1)$ & $107.5(2)$ \\
\hline $\mathrm{C}(9)-\mathrm{C}(1)-\mathrm{C}(5)$ & $111.5(2)$ \\
\hline $\mathrm{N}(1)-\mathrm{C}(1)-\mathrm{C}(5)$ & $112.1(2)$ \\
\hline $\mathrm{C}(9)-\mathrm{C}(1)-\mathrm{H}(1)$ & $108.7(12)$ \\
\hline $\mathrm{N}(1)-\mathrm{C}(1)-\mathrm{H}(1)$ & $104.7(12)$ \\
\hline $\mathrm{C}(5)-\mathrm{C}(1)-\mathrm{H}(1)$ & $111.9(12)$ \\
\hline $\mathrm{N}(1)-\mathrm{C}(2)-\mathrm{C}(3)$ & $108.6(2)$ \\
\hline $\mathrm{N}(1)-\mathrm{C}(2)-\mathrm{H}(2 \mathrm{~A})$ & $105.5(15)$ \\
\hline $\mathrm{C}(3)-\mathrm{C}(2)-\mathrm{H}(2 \mathrm{~A})$ & $111.8(15)$ \\
\hline $\mathrm{N}(1)-\mathrm{C}(2)-\mathrm{H}(2 \mathrm{~B})$ & $103.2(15)$ \\
\hline $\mathrm{C}(3)-\mathrm{C}(2)-\mathrm{H}(2 \mathrm{~B})$ & $111.5(16)$ \\
\hline $\mathrm{H}(2 \mathrm{~A})-\mathrm{C}(2)-\mathrm{H}(2 \mathrm{~B})$ & $115(2)$ \\
\hline $\mathrm{C}(2)-\mathrm{C}(3)-\mathrm{C}(4)$ & 111.1(2) \\
\hline $\mathrm{C}(2)-\mathrm{C}(3)-\mathrm{H}(3 \mathrm{~A})$ & $105(2)$ \\
\hline $\mathrm{C}(4)-\mathrm{C}(3)-\mathrm{H}(3 \mathrm{~A})$ & $109.8(19)$ \\
\hline $\mathrm{C}(2)-\mathrm{C}(3)-\mathrm{H}(3 \mathrm{~B})$ & $107.9(14)$ \\
\hline $\mathrm{C}(4)-\mathrm{C}(3)-\mathrm{H}(3 \mathrm{~B})$ & $110.2(14)$ \\
\hline $\mathrm{H}(3 \mathrm{~A})-\mathrm{C}(3)-\mathrm{H}(3 \mathrm{~B})$ & $113(2)$ \\
\hline $\mathrm{C}(3)-\mathrm{C}(4)-\mathrm{C}(5)$ & $114.1(2)$ \\
\hline $\mathrm{C}(3)-\mathrm{C}(4)-\mathrm{H}(4 \mathrm{~A})$ & $109.8(13)$ \\
\hline $\mathrm{C}(5)-\mathrm{C}(4)-\mathrm{H}(4 \mathrm{~A})$ & $106.3(13)$ \\
\hline $\mathrm{C}(3)-\mathrm{C}(4)-\mathrm{H}(4 \mathrm{~B})$ & $107.8(16)$ \\
\hline $\mathrm{C}(5)-\mathrm{C}(4)-\mathrm{H}(4 \mathrm{~B})$ & $110.4(16)$ \\
\hline $\mathrm{H}(4 \mathrm{~A})-\mathrm{C}(4)-\mathrm{H}(4 \mathrm{~B})$ & $108(2)$ \\
\hline
\end{tabular}




\begin{tabular}{|c|c|}
\hline$C(16)-C(5)-C(6)$ & $110.2(2)$ \\
\hline$C(16)-C(5)-C(4)$ & $108.8(2)$ \\
\hline$C(6)-C(5)-C(4)$ & 109.7(2) \\
\hline$C(16)-C(5)-C(1)$ & $108.1(2)$ \\
\hline$C(6)-C(5)-C(1)$ & $110.6(2)$ \\
\hline$C(4)-C(5)-C(1)$ & $109.4(2)$ \\
\hline$C(7)-C(6)-C(5)$ & $115.9(2)$ \\
\hline$C(7)-C(6)-H(6 A)$ & $103.7(14)$ \\
\hline$C(5)-C(6)-H(6 A)$ & $109.9(14)$ \\
\hline $\mathrm{C}(7)-\mathrm{C}(6)-\mathrm{H}(6 \mathrm{~B})$ & $109.7(14)$ \\
\hline $\mathrm{C}(5)-\mathrm{C}(6)-\mathrm{H}(6 \mathrm{~B})$ & $105.7(14)$ \\
\hline $\mathrm{H}(6 \mathrm{~A})-\mathrm{C}(6)-\mathrm{H}(6 \mathrm{~B})$ & 112(2) \\
\hline $\mathrm{O}(1)-\mathrm{C}(7)-\mathrm{C}(8)$ & $123.2(2)$ \\
\hline $\mathrm{O}(1)-\mathrm{C}(7)-\mathrm{C}(6)$ & $122.6(2)$ \\
\hline$C(8)-C(7)-C(6)$ & $114.2(2)$ \\
\hline $\mathrm{C}(9)-\mathrm{C}(8)-\mathrm{N}(2)$ & $110.0(2)$ \\
\hline $\mathrm{C}(9)-\mathrm{C}(8)-\mathrm{C}(7)$ & $125.3(2)$ \\
\hline $\mathrm{N}(2)-\mathrm{C}(8)-\mathrm{C}(7)$ & $124.7(2)$ \\
\hline$C(8)-C(9)-C(10)$ & $107.2(2)$ \\
\hline $\mathrm{C}(8)-\mathrm{C}(9)-\mathrm{C}(1)$ & $121.9(2)$ \\
\hline$C(10)-C(9)-C(1)$ & $130.8(2)$ \\
\hline$C(15)-C(10)-C(11)$ & $118.8(2)$ \\
\hline$C(15)-C(10)-C(9)$ & $135.2(3)$ \\
\hline $\mathrm{C}(11)-\mathrm{C}(10)-\mathrm{C}(9)$ & $106.1(2)$ \\
\hline $\mathrm{N}(2)-\mathrm{C}(11)-\mathrm{C}(12)$ & $129.5(3)$ \\
\hline $\mathrm{N}(2)-\mathrm{C}(11)-\mathrm{C}(10)$ & $108.4(2)$ \\
\hline $\mathrm{C}(12)-\mathrm{C}(11)-\mathrm{C}(10)$ & $122.0(2)$ \\
\hline$C(13)-C(12)-C(11)$ & $117.0(3)$ \\
\hline $\mathrm{C}(13)-\mathrm{C}(12)-\mathrm{H}(12)$ & $123.9(15)$ \\
\hline $\mathrm{C}(11)-\mathrm{C}(12)-\mathrm{H}(12)$ & $119.0(15)$ \\
\hline $\mathrm{C}(12)-\mathrm{C}(13)-\mathrm{C}(14)$ & $122.2(3)$ \\
\hline $\mathrm{C}(12)-\mathrm{C}(13)-\mathrm{H}(13)$ & $120.3(18)$ \\
\hline $\mathrm{C}(14)-\mathrm{C}(13)-\mathrm{H}(13)$ & $117.4(18)$ \\
\hline$C(15)-C(14)-C(13)$ & $120.8(3)$ \\
\hline $\mathrm{C}(15)-\mathrm{C}(14)-\mathrm{H}(14)$ & 117.9(17) \\
\hline $\mathrm{C}(13)-\mathrm{C}(14)-\mathrm{H}(14)$ & $121.3(17)$ \\
\hline $\mathrm{C}(14)-\mathrm{C}(15)-\mathrm{C}(10)$ & 119.1(3) \\
\hline $\mathrm{C}(14)-\mathrm{C}(15)-\mathrm{H}(15)$ & $121.5(15)$ \\
\hline
\end{tabular}




$\begin{array}{ll}\mathrm{C}(10)-\mathrm{C}(15)-\mathrm{H}(15) & 119.3(15) \\ \mathrm{C}(17)-\mathrm{C}(16)-\mathrm{C}(5) & 117.0(3) \\ \mathrm{C}(17)-\mathrm{C}(16)-\mathrm{H}(16 \mathrm{~A}) & 106.6(16) \\ \mathrm{C}(5)-\mathrm{C}(16)-\mathrm{H}(16 \mathrm{~A}) & 110.0(16) \\ \mathrm{C}(17)-\mathrm{C}(16)-\mathrm{H}(16 \mathrm{~B}) & 109.6(17) \\ \mathrm{C}(5)-\mathrm{C}(16)-\mathrm{H}(16 \mathrm{~B}) & 109.2(16) \\ \mathrm{H}(16 \mathrm{~A})-\mathrm{C}(16)-\mathrm{H}(16 \mathrm{~B}) & 104(2) \\ \mathrm{C}(16)-\mathrm{C}(17)-\mathrm{H}(17 \mathrm{~A}) & 109.4(17) \\ \mathrm{C}(16)-\mathrm{C}(17)-\mathrm{H}(17 \mathrm{~B}) & 111.3(17) \\ \mathrm{H}(17 \mathrm{~A})-\mathrm{C}(17)-\mathrm{H}(17 \mathrm{~B}) & 110(2) \\ \mathrm{C}(16)-\mathrm{C}(17)-\mathrm{H}(17 \mathrm{C}) & 110.1(17) \\ \mathrm{H}(17 \mathrm{~A})-\mathrm{C}(17)-\mathrm{H}(17 \mathrm{C}) & 108(2) \\ \mathrm{H}(17 \mathrm{~B})-\mathrm{C}(17)-\mathrm{H}(17 \mathrm{C}) & 108(2)\end{array}$

Symmetry transformations used to generate equivalent atoms:

Table 4. Anisotropic displacement parameters $\left(\AA^{2} \mathrm{x} 10^{3}\right)$ for fds 330 . The anisotropic displacement factor exponent takes the form: $-2 \pi^{2}\left[h^{2} a^{* 2} U^{11}+\ldots+2 h k a^{*} b^{*} U^{12}\right]$

\begin{tabular}{|c|c|c|c|c|c|c|}
\hline & $\mathrm{U}^{11}$ & $\mathrm{U}^{22}$ & $\mathrm{U}^{33}$ & $\mathrm{U}^{23}$ & $\mathrm{U}^{13}$ & $\mathrm{U}^{12}$ \\
\hline $\mathrm{Cl}(1)$ & $20(1)$ & $26(1)$ & $35(1)$ & $-2(1)$ & $7(1)$ & $-2(1)$ \\
\hline $\mathrm{O}(1)$ & $19(1)$ & $45(1)$ & $18(1)$ & $-11(1)$ & $3(1)$ & $-12(1)$ \\
\hline $\mathrm{N}(1)$ & $17(1)$ & $22(1)$ & $17(1)$ & $-1(1)$ & $2(1)$ & $-1(1)$ \\
\hline $\mathrm{N}(2)$ & $18(1)$ & $31(1)$ & $12(1)$ & $-5(1)$ & $3(1)$ & $-4(1)$ \\
\hline $\mathrm{C}(1)$ & $15(1)$ & $18(1)$ & $16(2)$ & $-2(1)$ & $2(1)$ & $-5(1)$ \\
\hline$C(2)$ & $29(2)$ & $26(2)$ & $12(2)$ & $-2(1)$ & 1(1) & $-2(1)$ \\
\hline $\mathrm{C}(3)$ & $26(2)$ & $34(2)$ & $11(2)$ & $-2(1)$ & $4(1)$ & $-4(1)$ \\
\hline $\mathrm{C}(4)$ & $20(2)$ & $26(2)$ & $17(2)$ & $-6(1)$ & $6(1)$ & $-6(1)$ \\
\hline$C(5)$ & $16(1)$ & $23(1)$ & $17(2)$ & $-3(1)$ & $5(1)$ & $-2(1)$ \\
\hline$C(6)$ & $16(2)$ & $22(2)$ & $17(2)$ & $-1(1)$ & $5(1)$ & $-2(1)$ \\
\hline$C(7)$ & $21(2)$ & $24(1)$ & $15(2)$ & $1(1)$ & $4(1)$ & 1(1) \\
\hline $\mathrm{C}(8)$ & $19(1)$ & $23(1)$ & $10(1)$ & $-1(1)$ & $4(1)$ & $-1(1)$ \\
\hline $\mathrm{C}(9)$ & $17(1)$ & 21(1) & $14(1)$ & $1(1)$ & $6(1)$ & $1(1)$ \\
\hline$C(10)$ & $17(1)$ & $20(1)$ & $15(1)$ & $4(1)$ & $3(1)$ & $4(1)$ \\
\hline $\mathrm{C}(11)$ & $17(1)$ & $20(1)$ & $17(1)$ & $3(1)$ & $1(1)$ & $4(1)$ \\
\hline$C(12)$ & $25(2)$ & $30(2)$ & $15(2)$ & 1(1) & $7(1)$ & $5(1)$ \\
\hline$C(13)$ & $24(2)$ & $34(2)$ & $28(2)$ & $6(1)$ & $18(2)$ & $7(1)$ \\
\hline
\end{tabular}




\begin{tabular}{lllllll}
$\mathrm{C}(14)$ & $19(2)$ & $31(2)$ & $29(2)$ & $4(1)$ & $9(1)$ & $-1(1)$ \\
$\mathrm{C}(15)$ & $17(2)$ & $26(2)$ & $21(2)$ & $0(1)$ & $4(1)$ & $-1(1)$ \\
$\mathrm{C}(16)$ & $24(2)$ & $23(2)$ & $22(2)$ & $-1(1)$ & $5(1)$ & $-3(1)$ \\
$\mathrm{C}(17)$ & $23(2)$ & $31(2)$ & $29(2)$ & $-1(2)$ & $7(2)$ & $1(2)$ \\
\hline
\end{tabular}

Table 5. Hydrogen coordinates ( $\left.\mathrm{x} 10^{4}\right)$ and isotropic displacement parameters $\left(\AA^{2} \times 10^{3}\right)$ for fds 330 .

\begin{tabular}{|c|c|c|c|c|}
\hline & $\mathrm{x}$ & $\mathrm{y}$ & $\mathrm{z}$ & $\mathrm{U}(\mathrm{eq})$ \\
\hline $\mathrm{H}(1 \mathrm{~A})$ & $3400(30)$ & $5384(17)$ & $4680(20)$ & $10(6)$ \\
\hline $\mathrm{H}(1 \mathrm{~B})$ & $4760(40)$ & $5960(20)$ & $4780(30)$ & $50(10)$ \\
\hline $\mathrm{H}(2)$ & $1480(30)$ & $5340(20)$ & $120(30)$ & $40(10)$ \\
\hline $\mathrm{H}(1)$ & $3410(20)$ & $7140(14)$ & $4114(19)$ & $0(5)$ \\
\hline $\mathrm{H}(2 \mathrm{~A})$ & $4220(30)$ & 6627(17) & $6390(20)$ & $17(7)$ \\
\hline $\mathrm{H}(2 \mathrm{~B})$ & $4290(30)$ & $5608(17)$ & $6590(20)$ & $15(7)$ \\
\hline $\mathrm{H}(3 \mathrm{~A})$ & $2230(30)$ & $6205(18)$ & $7120(30)$ & $33(9)$ \\
\hline $\mathrm{H}(3 \mathrm{~B})$ & $1730(30)$ & 5493(17) & $6090(20)$ & $12(6)$ \\
\hline $\mathrm{H}(4 \mathrm{~A})$ & $1660(30)$ & $7341(16)$ & $5750(20)$ & $9(6)$ \\
\hline $\mathrm{H}(4 \mathrm{~B})$ & $250(30)$ & 6741(17) & $5630(20)$ & $23(7)$ \\
\hline $\mathrm{H}(6 \mathrm{~A})$ & $660(30)$ & $5355(17)$ & $4050(20)$ & $14(7)$ \\
\hline $\mathrm{H}(6 \mathrm{~B})$ & $-750(30)$ & $6003(16)$ & $3590(20)$ & 16(7) \\
\hline $\mathrm{H}(12)$ & $3810(30)$ & $5644(16)$ & $-930(20)$ & $13(7)$ \\
\hline $\mathrm{H}(13)$ & $6110(30)$ & $6345(16)$ & $-460(20)$ & $15(7)$ \\
\hline $\mathrm{H}(14)$ & $7100(30)$ & $7047(18)$ & $1370(30)$ & $28(8)$ \\
\hline $\mathrm{H}(15)$ & $5840(30)$ & $7044(16)$ & $2990(20)$ & 20(7) \\
\hline $\mathrm{H}(16 \mathrm{~A})$ & $1240(30)$ & 7961(18) & $3560(20)$ & $28(8)$ \\
\hline $\mathrm{H}(16 \mathrm{~B})$ & $350(30)$ & $7405(16)$ & $2520(30)$ & $24(8)$ \\
\hline $\mathrm{H}(17 \mathrm{~A})$ & $-1560(30)$ & $7390(17)$ & $3700(20)$ & $21(8)$ \\
\hline $\mathrm{H}(17 \mathrm{~B})$ & $-1360(30)$ & $8270(19)$ & $3130(30)$ & $28(8)$ \\
\hline $\mathrm{H}(17 \mathrm{C})$ & $-670(30)$ & $8095(18)$ & $4530(30)$ & $26(8)$ \\
\hline
\end{tabular}

Table 6. Torsion angles $\left[^{\circ}\right]$ for fds 330 . 


\begin{tabular}{|c|c|}
\hline $\mathrm{C}(2)-\mathrm{N}(1)-\mathrm{C}(1)-\mathrm{C}(5)$ & $-52.9(3)$ \\
\hline $\mathrm{C}(1)-\mathrm{N}(1)-\mathrm{C}(2)-\mathrm{C}(3)$ & $57.1(3)$ \\
\hline $\mathrm{N}(1)-\mathrm{C}(2)-\mathrm{C}(3)-\mathrm{C}(4)$ & $-57.6(3)$ \\
\hline$C(2)-C(3)-C(4)-C(5)$ & $57.6(3)$ \\
\hline$C(3)-C(4)-C(5)-C(16)$ & $-168.6(2)$ \\
\hline$C(3)-C(4)-C(5)-C(6)$ & $70.7(3)$ \\
\hline$C(3)-C(4)-C(5)-C(1)$ & $-50.8(3)$ \\
\hline $\mathrm{C}(9)-\mathrm{C}(1)-\mathrm{C}(5)-\mathrm{C}(16)$ & $-74.1(3)$ \\
\hline $\mathrm{N}(1)-\mathrm{C}(1)-\mathrm{C}(5)-\mathrm{C}(16)$ & $165.3(2)$ \\
\hline$C(9)-C(1)-C(5)-C(6)$ & $46.6(3)$ \\
\hline$N(1)-C(1)-C(5)-C(6)$ & $-74.0(3)$ \\
\hline$C(9)-C(1)-C(5)-C(4)$ & $167.5(2)$ \\
\hline $\mathrm{N}(1)-\mathrm{C}(1)-\mathrm{C}(5)-\mathrm{C}(4)$ & $46.9(3)$ \\
\hline $\mathrm{C}(16)-\mathrm{C}(5)-\mathrm{C}(6)-\mathrm{C}(7)$ & $68.6(3)$ \\
\hline$C(4)-C(5)-C(6)-C(7)$ & $-171.6(2)$ \\
\hline$C(1)-C(5)-C(6)-C(7)$ & $-50.9(3)$ \\
\hline $\mathrm{C}(5)-\mathrm{C}(6)-\mathrm{C}(7)-\mathrm{O}(1)$ & $-153.3(3)$ \\
\hline$C(5)-C(6)-C(7)-C(8)$ & $29.2(3)$ \\
\hline $\mathrm{C}(11)-\mathrm{N}(2)-\mathrm{C}(8)-\mathrm{C}(9)$ & $0.5(3)$ \\
\hline $\mathrm{C}(11)-\mathrm{N}(2)-\mathrm{C}(8)-\mathrm{C}(7)$ & $178.7(2)$ \\
\hline $\mathrm{O}(1)-\mathrm{C}(7)-\mathrm{C}(8)-\mathrm{C}(9)$ & $178.3(3)$ \\
\hline $\mathrm{C}(6)-\mathrm{C}(7)-\mathrm{C}(8)-\mathrm{C}(9)$ & $-4.2(4)$ \\
\hline $\mathrm{O}(1)-\mathrm{C}(7)-\mathrm{C}(8)-\mathrm{N}(2)$ & $0.3(4)$ \\
\hline $\mathrm{C}(6)-\mathrm{C}(7)-\mathrm{C}(8)-\mathrm{N}(2)$ & $177.8(2)$ \\
\hline $\mathrm{N}(2)-\mathrm{C}(8)-\mathrm{C}(9)-\mathrm{C}(10)$ & $-0.6(3)$ \\
\hline $\mathrm{C}(7)-\mathrm{C}(8)-\mathrm{C}(9)-\mathrm{C}(10)$ & $-178.9(2)$ \\
\hline $\mathrm{N}(2)-\mathrm{C}(8)-\mathrm{C}(9)-\mathrm{C}(1)$ & $-179.2(2)$ \\
\hline $\mathrm{C}(7)-\mathrm{C}(8)-\mathrm{C}(9)-\mathrm{C}(1)$ & $2.6(4)$ \\
\hline $\mathrm{N}(1)-\mathrm{C}(1)-\mathrm{C}(9)-\mathrm{C}(8)$ & $98.6(3)$ \\
\hline$C(5)-C(1)-C(9)-C(8)$ & $-24.6(3)$ \\
\hline $\mathrm{N}(1)-\mathrm{C}(1)-\mathrm{C}(9)-\mathrm{C}(10)$ & $-79.6(3)$ \\
\hline $\mathrm{C}(5)-\mathrm{C}(1)-\mathrm{C}(9)-\mathrm{C}(10)$ & $157.2(3)$ \\
\hline$C(8)-C(9)-C(10)-C(15)$ & $179.4(3)$ \\
\hline$C(1)-C(9)-C(10)-C(15)$ & $-2.2(5)$ \\
\hline$C(8)-C(9)-C(10)-C(11)$ & $0.5(3)$ \\
\hline$C(1)-C(9)-C(10)-C(11)$ & $178.9(2)$ \\
\hline $\mathrm{C}(8)-\mathrm{N}(2)-\mathrm{C}(11)-\mathrm{C}(12)$ & $-179.1(3)$ \\
\hline $\mathrm{C}(8)-\mathrm{N}(2)-\mathrm{C}(11)-\mathrm{C}(10)$ & $-0.1(3)$ \\
\hline
\end{tabular}




$\begin{array}{lc}\mathrm{C}(15)-\mathrm{C}(10)-\mathrm{C}(11)-\mathrm{N}(2) & -179.4(2) \\ \mathrm{C}(9)-\mathrm{C}(10)-\mathrm{C}(11)-\mathrm{N}(2) & -0.3(3) \\ \mathrm{C}(15)-\mathrm{C}(10)-\mathrm{C}(11)-\mathrm{C}(12) & -0.3(4) \\ \mathrm{C}(9)-\mathrm{C}(10)-\mathrm{C}(11)-\mathrm{C}(12) & 178.8(2) \\ \mathrm{N}(2)-\mathrm{C}(11)-\mathrm{C}(12)-\mathrm{C}(13) & 178.7(3) \\ \mathrm{C}(10)-\mathrm{C}(11)-\mathrm{C}(12)-\mathrm{C}(13) & -0.2(4) \\ \mathrm{C}(11)-\mathrm{C}(12)-\mathrm{C}(13)-\mathrm{C}(14) & 0.4(4) \\ \mathrm{C}(12)-\mathrm{C}(13)-\mathrm{C}(14)-\mathrm{C}(15) & 0.0(4) \\ \mathrm{C}(13)-\mathrm{C}(14)-\mathrm{C}(15)-\mathrm{C}(10) & -0.5(4) \\ \mathrm{C}(11)-\mathrm{C}(10)-\mathrm{C}(15)-\mathrm{C}(14) & 0.7(4) \\ \mathrm{C}(9)-\mathrm{C}(10)-\mathrm{C}(15)-\mathrm{C}(14) & -178.2(3) \\ \mathrm{C}(6)-\mathrm{C}(5)-\mathrm{C}(16)-\mathrm{C}(17) & 74.6(3) \\ \mathrm{C}(4)-\mathrm{C}(5)-\mathrm{C}(16)-\mathrm{C}(17) & -45.7(3) \\ \mathrm{C}(1)-\mathrm{C}(5)-\mathrm{C}(16)-\mathrm{C}(17) & -164.4(3)\end{array}$

Symmetry transformations used to generate equivalent atoms:

Table 7. Hydrogen bonds for fds330 [ $\left[\AA\right.$ and ${ }^{\circ}$.

\begin{tabular}{lcccc}
\hline $\mathrm{D}-\mathrm{H} \ldots \mathrm{A}$ & $\mathrm{d}(\mathrm{D}-\mathrm{H})$ & $\mathrm{d}(\mathrm{H} \ldots \mathrm{A})$ & $\mathrm{d}(\mathrm{D} \ldots \mathrm{A})$ & $<(\mathrm{DHA})$ \\
\hline $\mathrm{N}(1)-\mathrm{H}(1 \mathrm{~A}) \ldots \mathrm{Cl}(1)$ & $0.91(3)$ & $2.19(3)$ & $3.082(3)$ & $170(2)$ \\
$\mathrm{N}(1)-\mathrm{H}(1 \mathrm{~B}) \ldots \mathrm{Cl}(1) \# 1$ & $0.95(3)$ & $2.17(3)$ & $3.069(2)$ & $159(3)$ \\
$\mathrm{N}(2)-\mathrm{H}(2) \ldots \mathrm{O}(1) \# 2$ & $0.88(3)$ & $1.98(3)$ & $2.841(3)$ & $164(3)$ \\
\hline
\end{tabular}

Symmetry transformations used to generate equivalent atoms:

$\# 1-x+1,-y+1,-z+1 \quad \# 2-x,-y+1,-z$ 


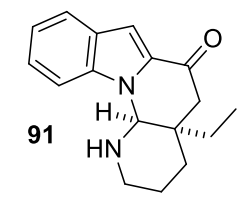

$\mathrm{CD}_{3} \mathrm{OD}$

$400 \mathrm{MHz}$

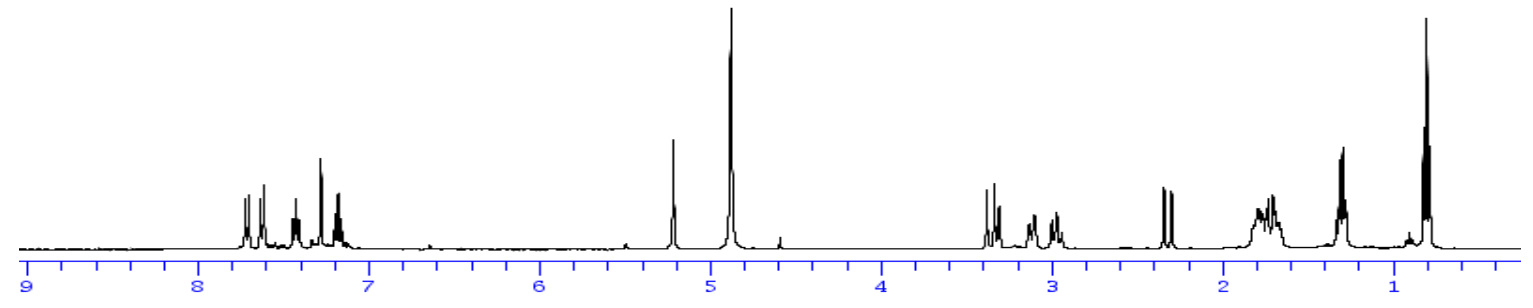

$\mathrm{CD}_{3} \mathrm{OD}$

$125 \mathrm{MHz}$
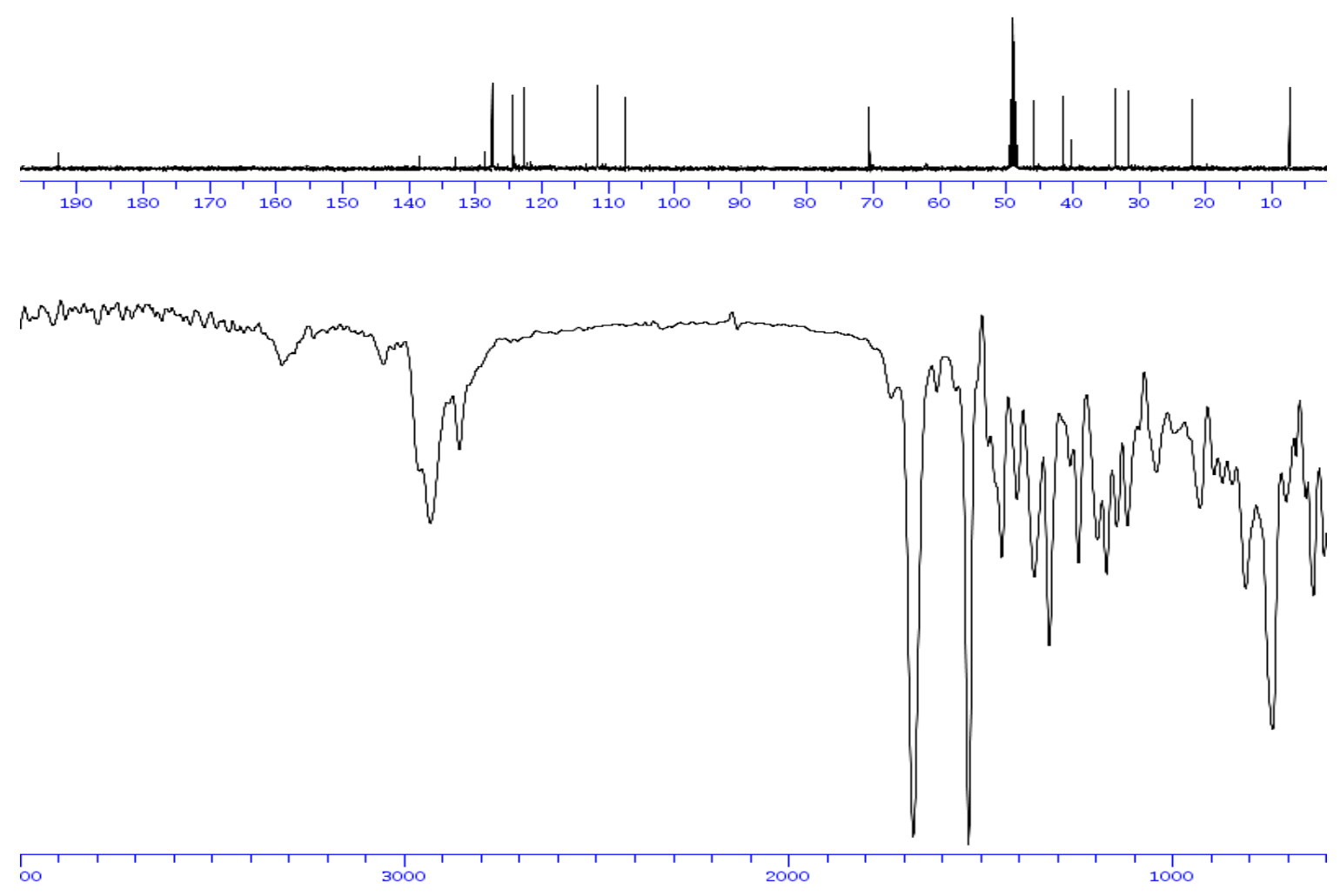

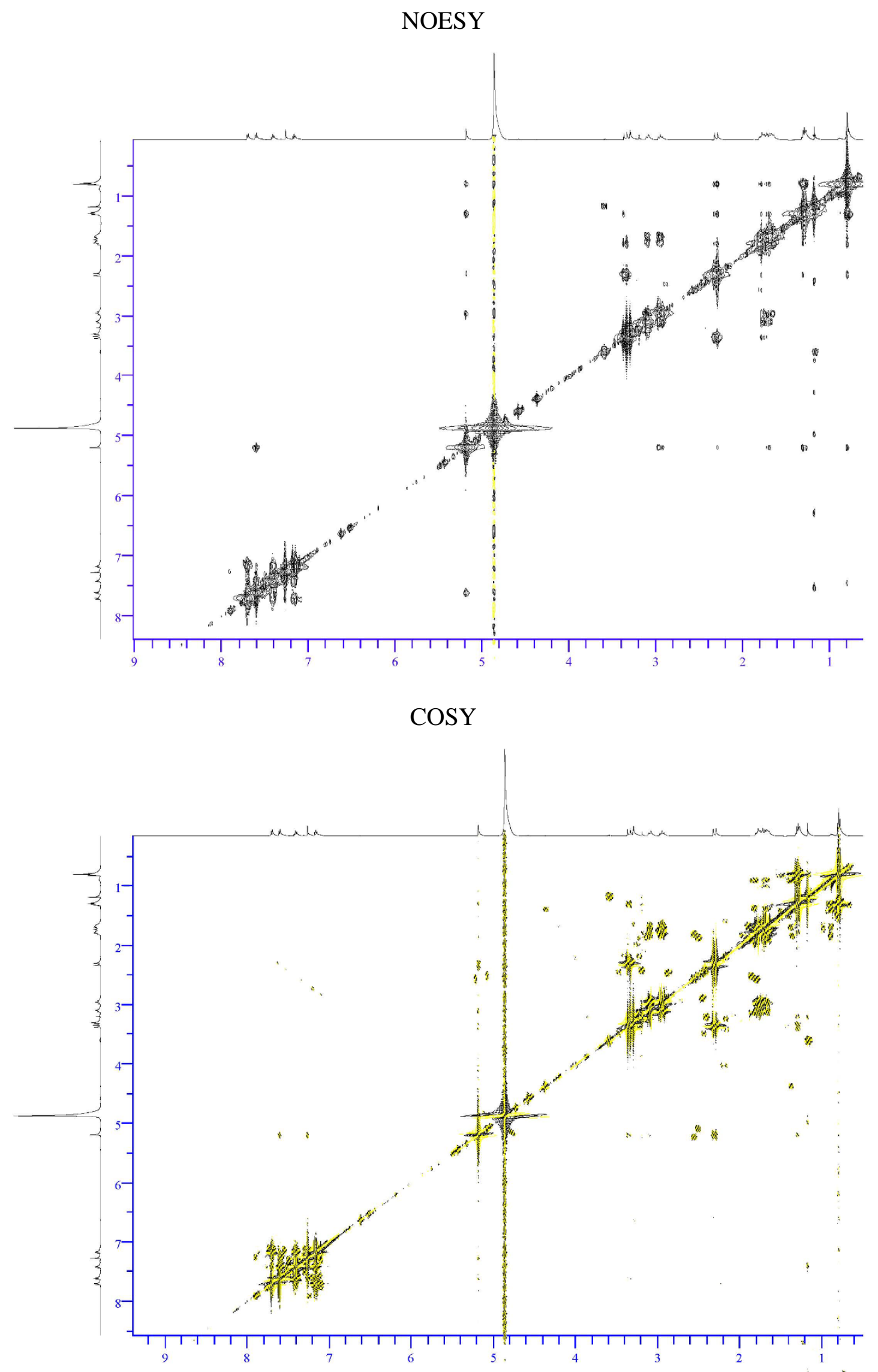

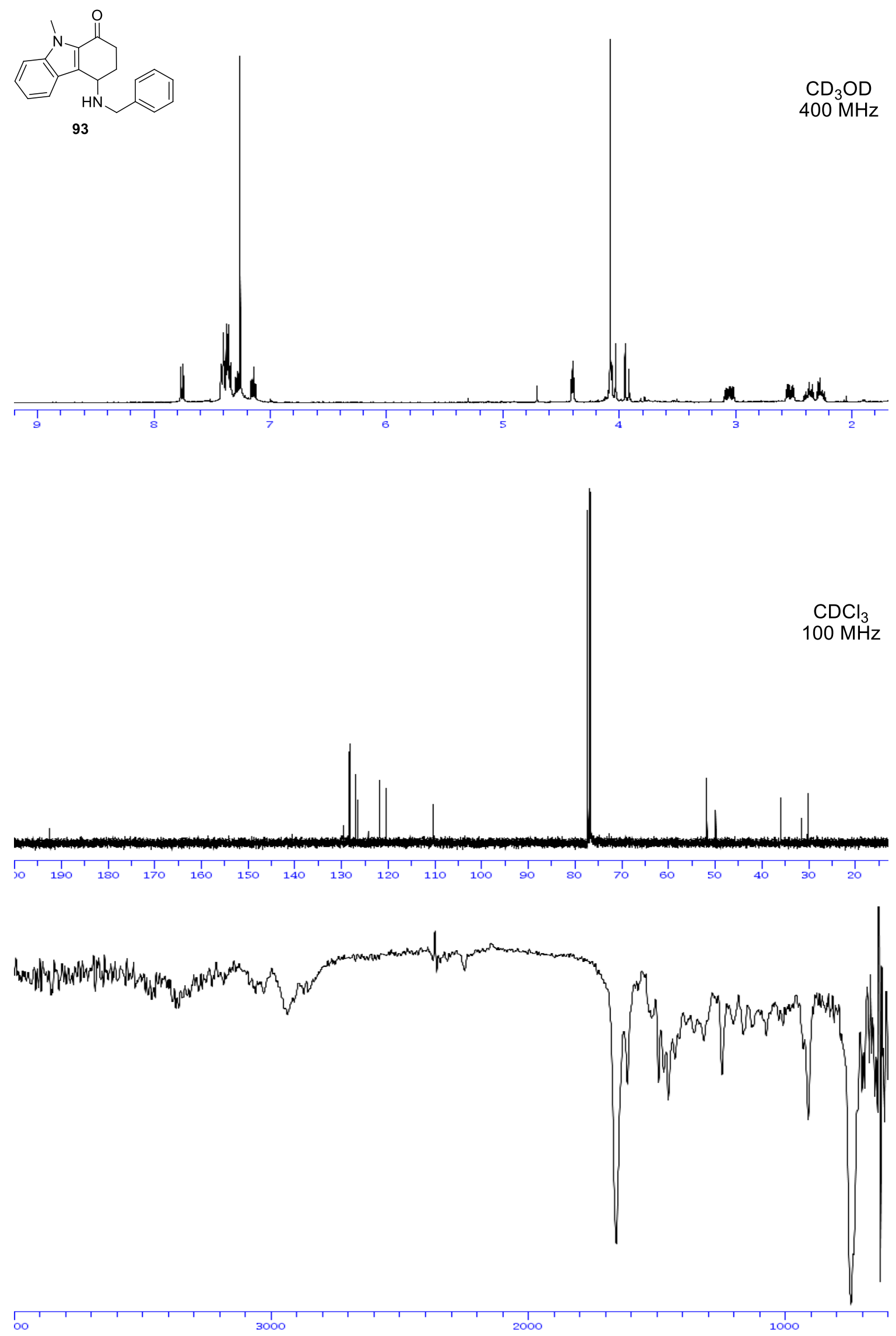